\title{
ECLAMPSIA IN THE UNITED KINGDOM
}

\section{KIRSTY A DOUGLAS}

Department of Obstetrics and Gynaecology

University of Adelaide

June 1995 


\section{INDEX}

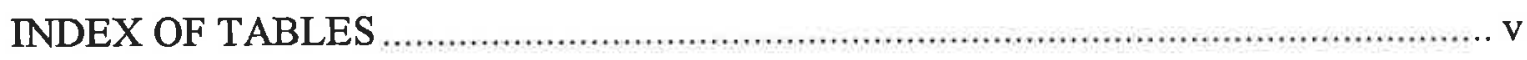

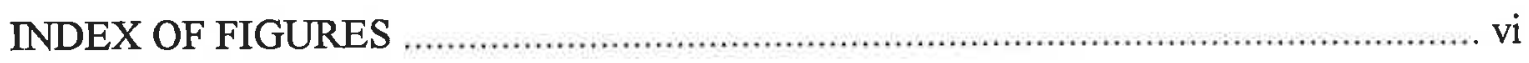

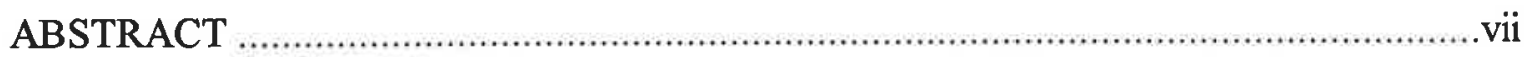

STATEMENT OF ORIGINALITY ................................................................................

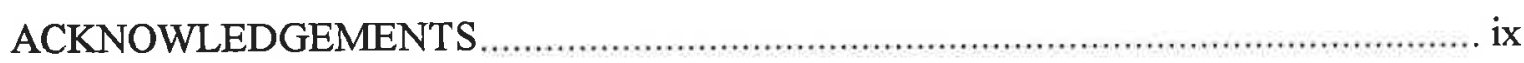

CHAPTER 1. LITERATURE REVIEW 1

1.1. FIRST DESCRIPTIONS- HISTORICAL PERSPECTIVE .................................. 2

1.2. CLASSIFICATION AND DEFINITIONS OF PRE-ECLAMPSIA AND

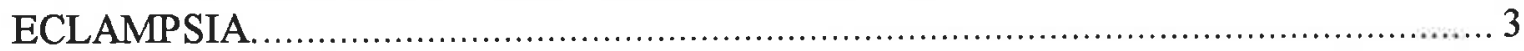

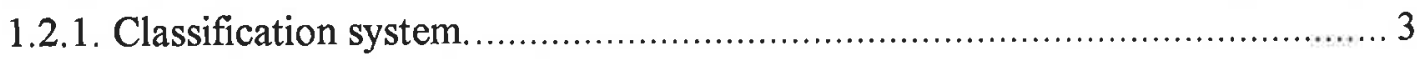

1.2.2. Definition of pre-eclampsia........................................................... 3

1.2.3. Definition of eclampsia. ............................................................. 5

1.3. THE EPIDEMIOLOGY OF ECLAMPSIA. ................................................. 5

1.3.1. The difficulties in studying the epidemiology of eclampsia ....................... 5

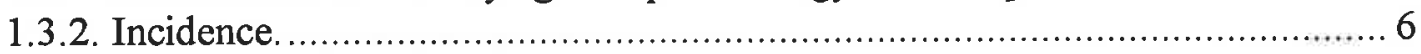

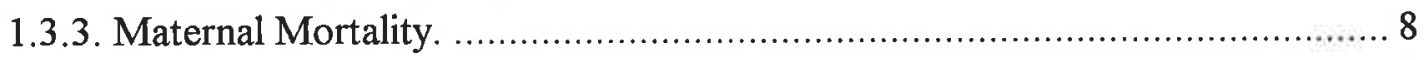

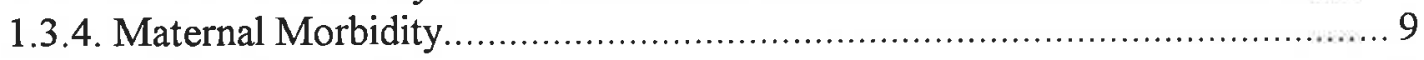

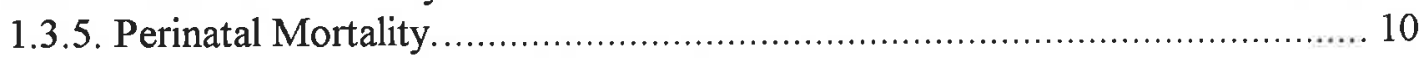

1.3.6. Risk factors associated with pre-eclampsia and eclampsia. ...................... 10

1.4. PATHOGENESIS OF PRE-ECLAMPSIA AND ECLAMPSIA ........................ 17

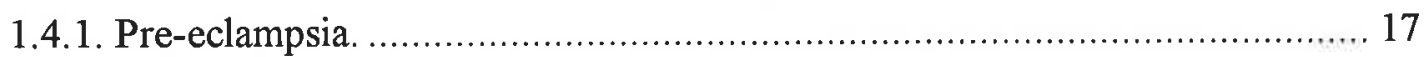

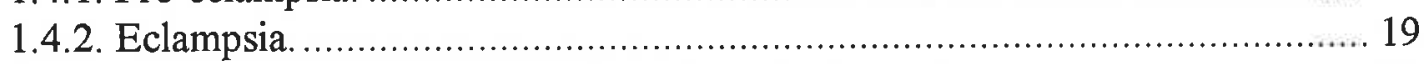

1.4.3. Relationship between pre-eclampsia and eclampsia. .............................. 20

1.4.4. Complications of pre-eclampsia and eclampsia............................... 21

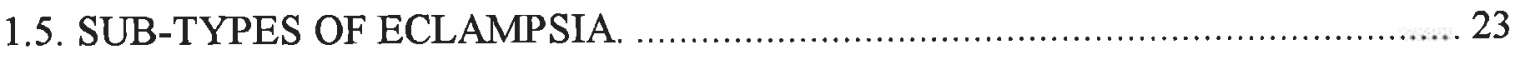

1.5.1. Timing of seizures in relation to the onset of labour. ............................. 23

1.5.2. Gestational age at onset of seizures............................................... 24

1.6. MANAGEMENT OF ECLAMPSIA AND PRE-ECLAMPSIA ......................... 25

1.6.1. Current methods and controversies.................................................. 25

1.6.2. Possible preventive measures .................................................... 27

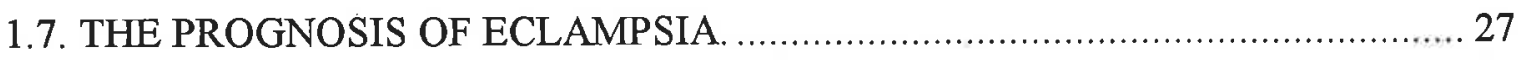

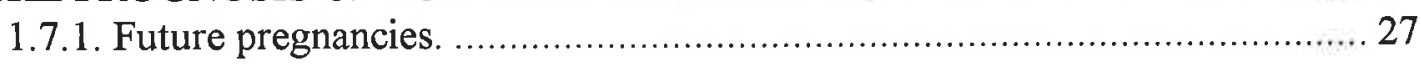

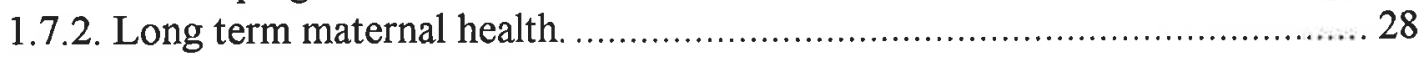

1.8. THE NEED FOR A NATIONAL SURVEY. ........................................... 28

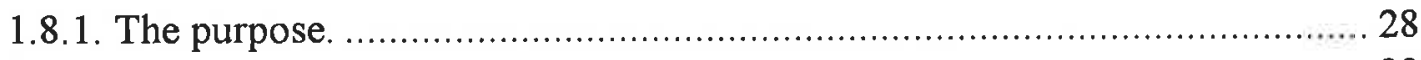

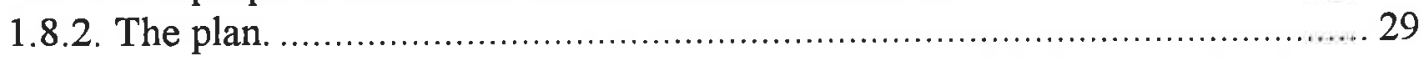

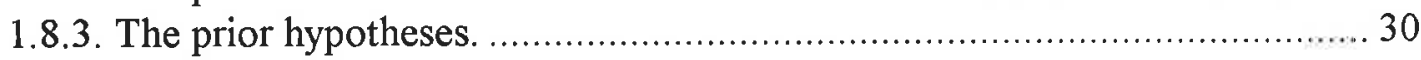




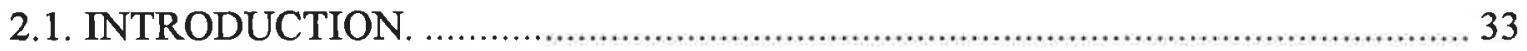

2.2. METHODS.

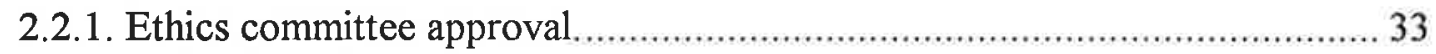

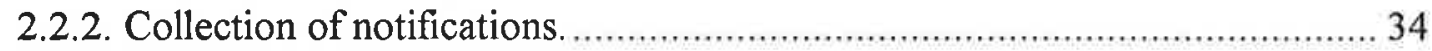

2.2.3. Collection of clinical data................................................................ 36

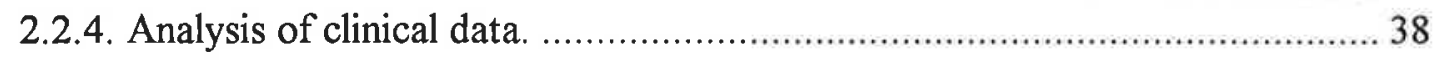

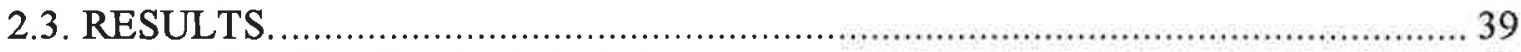

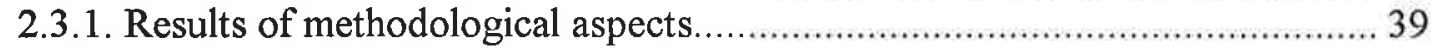

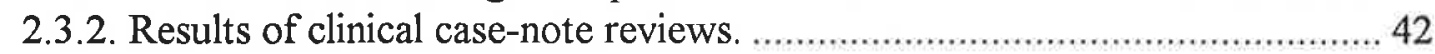

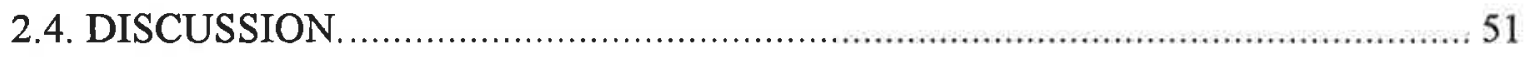

2.5. LESSONS FROM THE PILOT STUDY ON METHODOLOGY..........................53

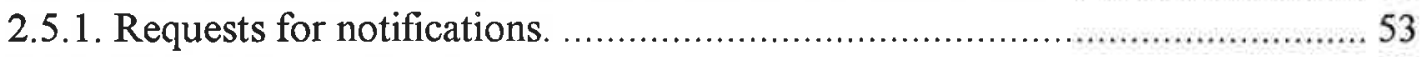

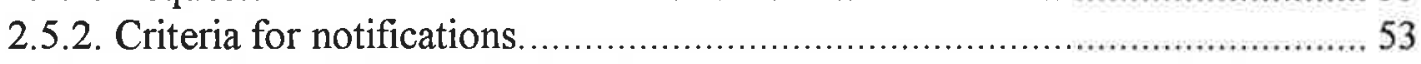

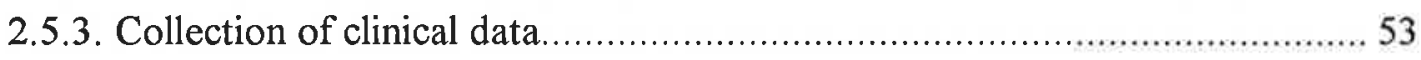

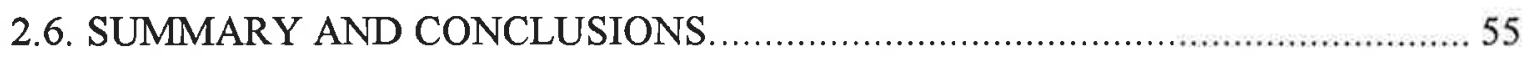

CHAPTER 3. METHODOLOGY 56

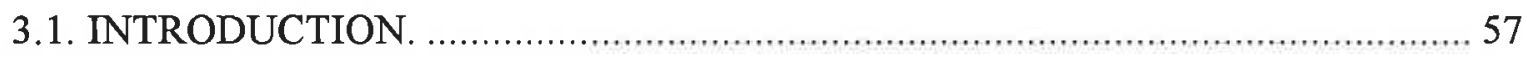

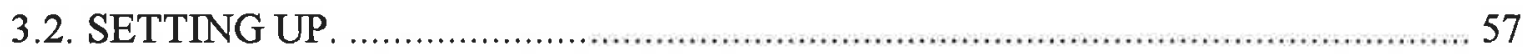

3.2.1. Support from professional bodies........................................................ 57

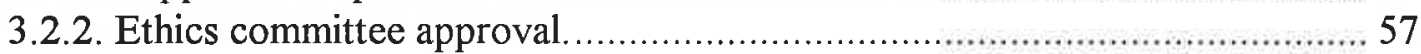

3.2.3. Establishing consultant lists and midwife contacts.................................59

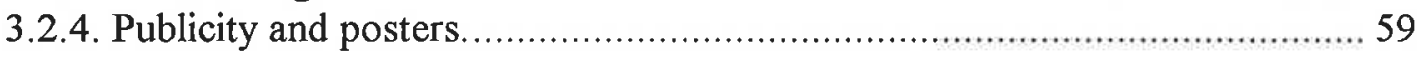

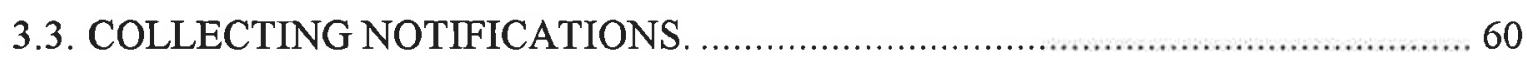

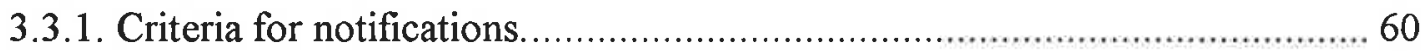

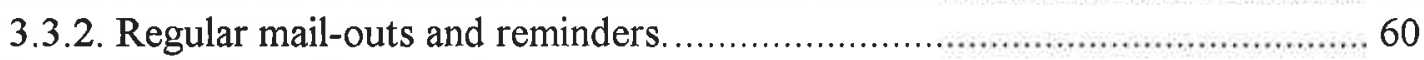

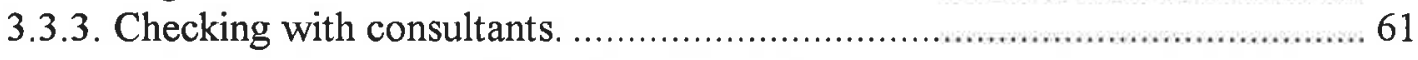

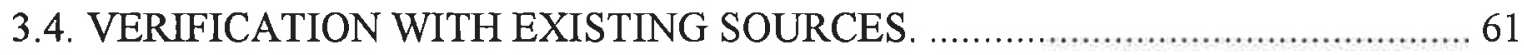

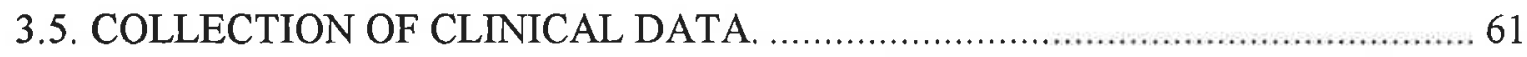

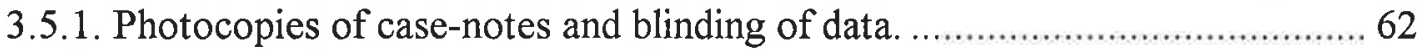

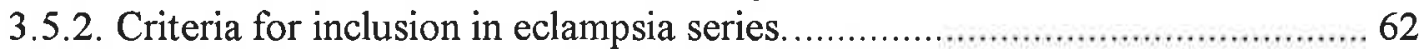

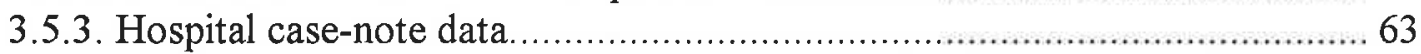

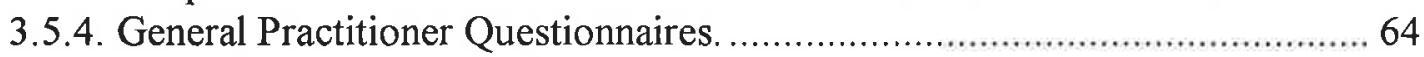

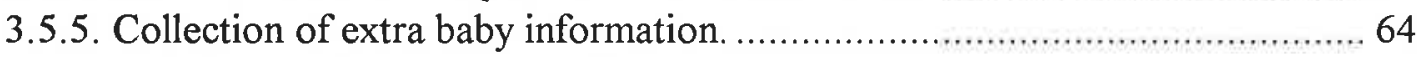

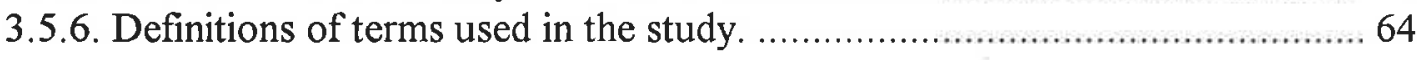

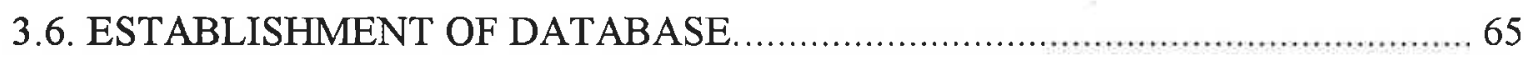

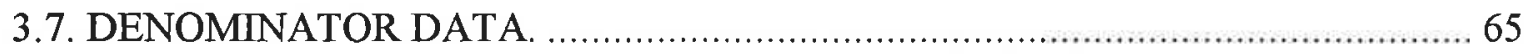

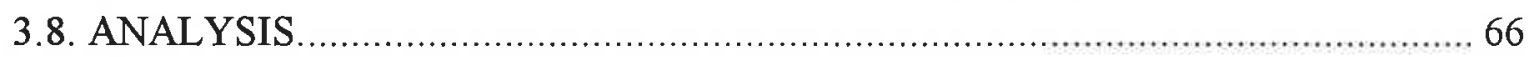

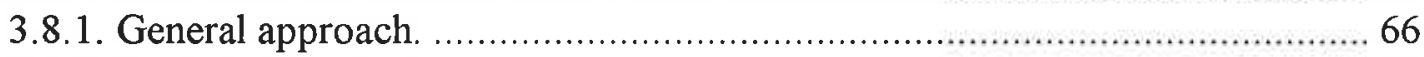

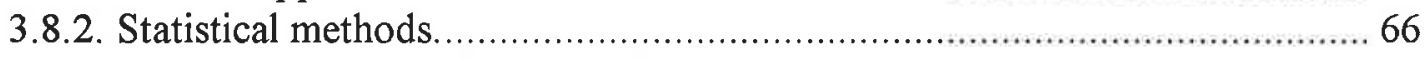

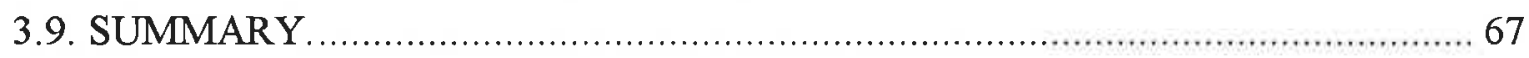


4.1. NOTIFICATION AND DATA COLLECTION............................................... 70

4.1.1. Response rates to requests for notifications........................................... 70

4.1.2. Degree of over notification. ....................................................... 70

4.1.3. Double notification of eclamptic cases. ................................................... 71

4.1.4. Comparison with existing maternity data systems ....................................... 73

4.1.5. Hospital case-notes reviewed............................................................... 73

4.1.6. Extra case-notes requested and reviewed. ............................................... 74

4.1.7. General Practitioner Questionnaires received. ............................................. 74

4.1.8. Complete antenatal care records. .................................................. 74

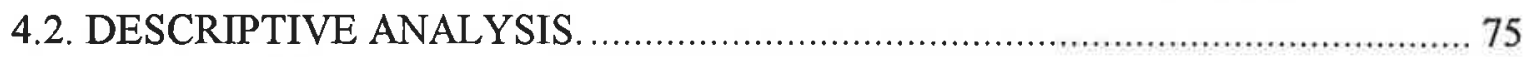

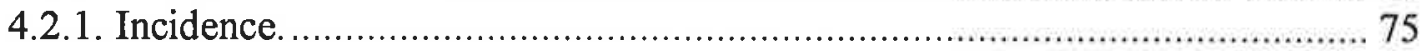

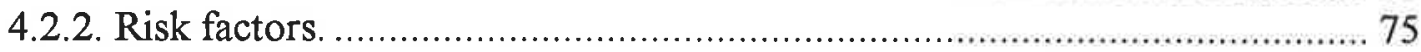

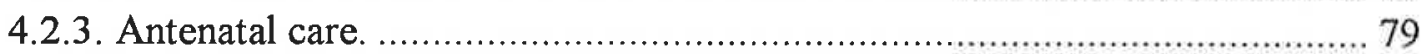

4.2.4. Location of patient at time of first seizure.......................................... 79

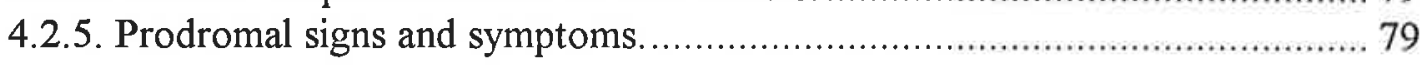

4.2.6. Antepartum, Intrapartum, Postpartum. ................................................ 82

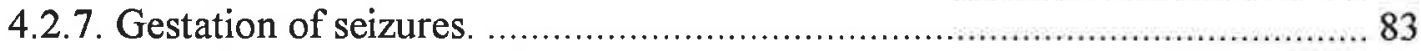

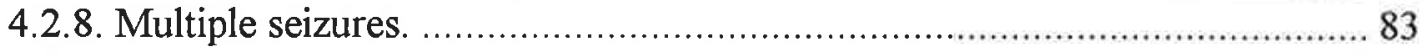

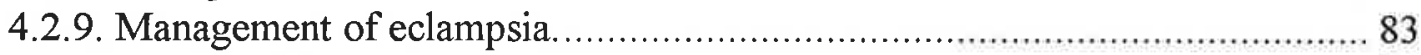

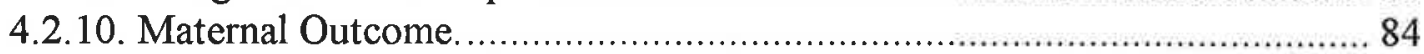

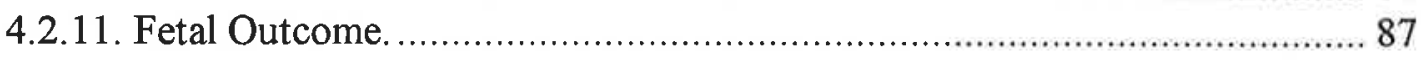

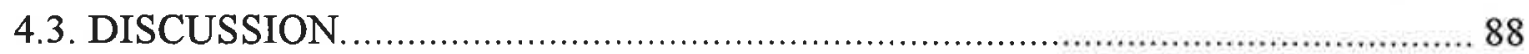

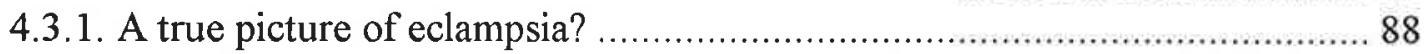

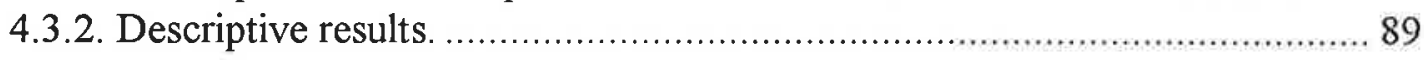

\section{CHAPTER 5. \\ SUBGROUP ANALYSIS AND LOGISTIC REGRESSION 93}

5.1. ANTENATAL CARE - WHAT IS ITS ROLE? ............................................... 94

5.1.1. Prior hypothesis: A significant proportion of eclampsia occurs in women who have had a long interval between formal screening checks for the signs of pre-eclampsia.

5.1.2. Further questions: Do women who have long antenatal screening intervals prior to the onset of eclampsia develop more complications than those women who receive frequent screening for pre-eclampsia? ......................... 95

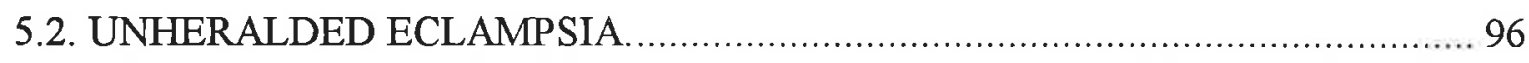

5.2.1. Prior hypothesis: Eclampsia occurs in some women without prodromal

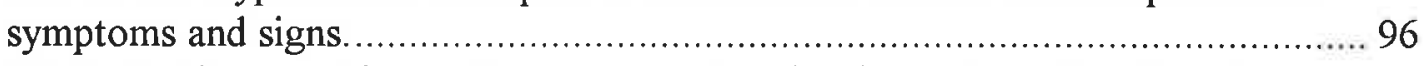

5.2.2. Further questions: Do women who develop eclampsia without the classical prodromal illness have a fewer complications? ...................................... 96

5.3. ECLAMPSIA AT THE EXTREMES OF MATERNAL AGE ………................. 99

5.3.1. Prior hypothesis: There is greater morbidity from eclampsia amongst

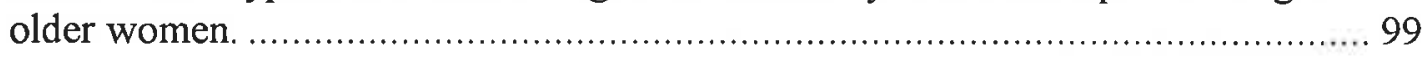

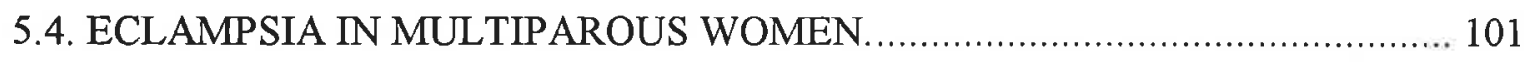


5.4.1. Prior hypothesis: A significant proportion of eclampsia occurs in multiparous women and these women have a greater morbidity from eclampsia

5.5. ANTEPARTUM, INTRAPARTUM AND POSTPARTUM ECLAMPSIA. ........ 103 5.5.1. Hypothesis arising from pilot study: Eclampsia occurring prior to labour is a more severe form of eclampsia than intra- or postpartum eclampsia. .. 103 5.5.2. Prior hypothesis: Post-partum eclampsia which occurs without preexisting signs of severe pre-eclampsia is a relatively benign condition............... 104

5.6. EARLY ONSET PRE-ECLAMPSIA - IS IT A MORE SEVERE ILLNESS? ...... 107 5.6.1. Hypothesis arising from pilot study: Pre-term eclampsia has more severe consequences for mother and fetus than eclampsia which occurs at term.

5.6.2. Prior hypothesis: A significant proportion of eclampsia occurs in women at or before 32 weeks gestation. .................................................. 108

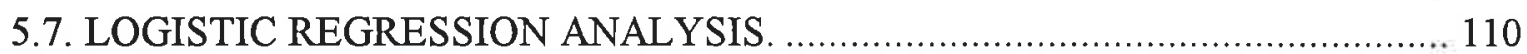

5.7.1. Introduction to multiple logistic regression analysis........................... 110

5.7.2. Associations with major maternal complications. ............................. 112

5.7.3. Associations with general practitioner reported maternal morbidity......... 113

5.7.4. Associations with fetal/infant death.................................................... 114

5.7.5. Associations with infant morbidity - multiple logistic regression analysis ... 115

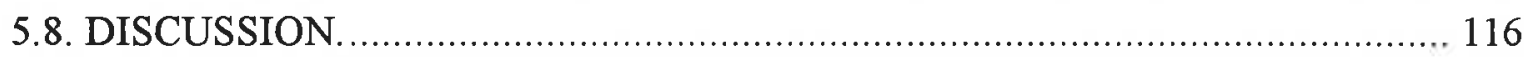

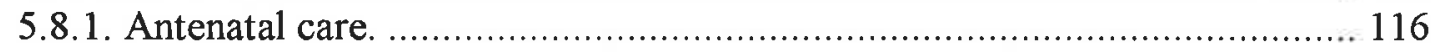

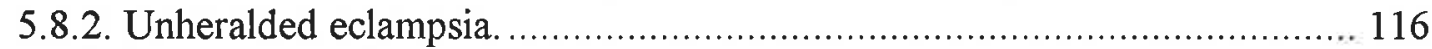

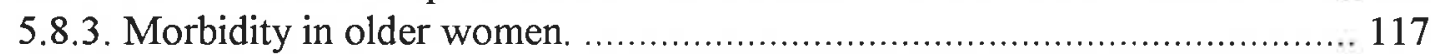

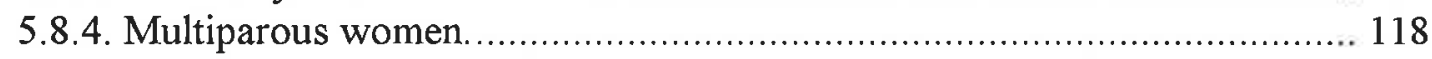

5.8.5. Antepartum vs. intra- and postpartum convulsions. ............................. 118

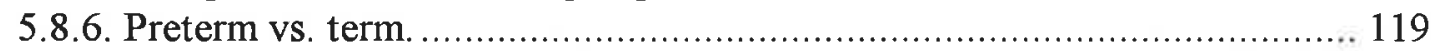

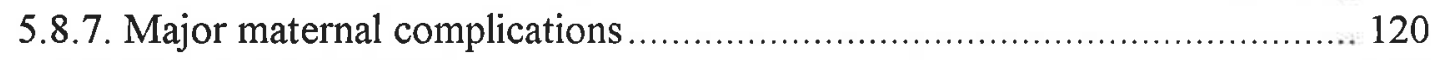

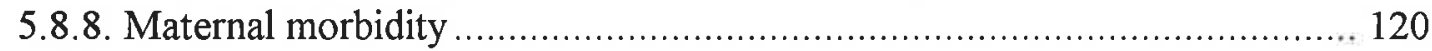

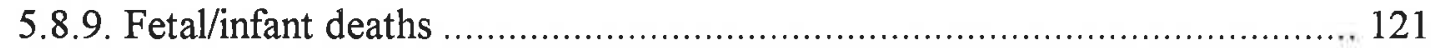

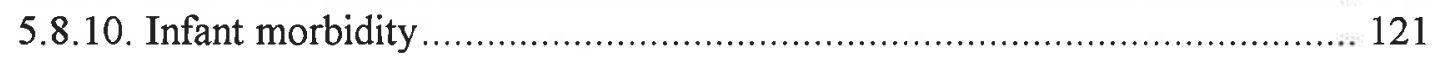

CHAPTER 6. CONCLUSIONS 123

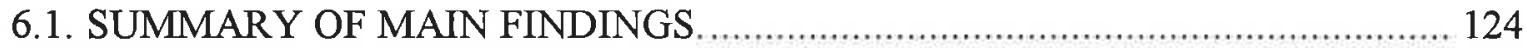

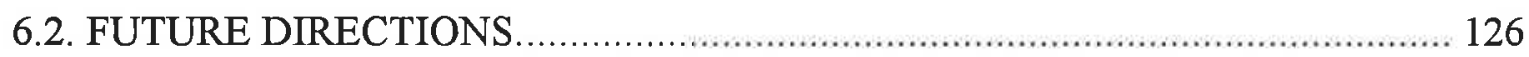

APPENDICES

Data sheet

General Practitioner questionnaire

Publications arising from the thesis 


\section{INDEX OF TABLES}

Table 1.1. Classification of hypertensive disorders of pregnancy.......................... 4

Table 1.2. Risk factors for pre-eclampsia and eclampsia. .................................... 11

Table 1.3. Proportion of primigravidae in published series of eclampsia. ................. 11

Table 1.4. Severe complications and acute crises in pre-eclampsia and

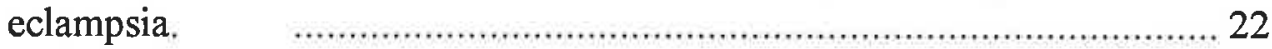

Table 1.5. The differential diagnoses of eclampsia. ............................................... 24

Table 1.6. Aims and prior hypotheses of a national descriptive study of

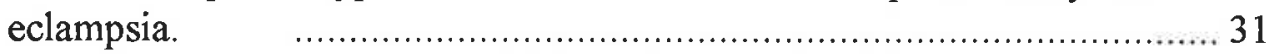

Table 2.1. Pilot study: Responses of consultants and midwives to mail-outs............ 39

Table 2.2. Pilot study: The incidence of eclampsia in the Oxford and the North-Western RHA's.

Table 2.3. Pilot study: Features of antenatal care of women with eclampsia.

Table 2.4. Pilot study: Number of patients with eclampsia by gestational age and type of eclampsia.

Table 2.5. Pilot study: Investigations carried out before and after first eclamptic seizures.

Table 2.6. Pilot study: Medications used in the management of women with eclampsia.

Table 2.7. Pilot study: Major complications of women with eclampsia .................. 48

Table 2.8. Pilot study: Maternal outcomes by gestation at onset of eclampsia ......... 49

Table 2.9. Pilot study: Maternal outcomes by type of eclampsia............................ 49

Table 2.10. Pilot study: Outcomes of singletons by gestational age at onset of eclamptic seizure. ..................................................................... 50

Table 2.11. Pilot study: Outcomes of singletons by type of eclampsia...................... 51

Table 4.1. The overall response rates to requests for notifications by regions........... 71

Table 4.2. Responses rates to each request for notifications................................ 72

Table 4.3. Validation of notification system by comparison with established maternity data systems. ............................................................... 73

Table 4.4. Incidence of Eclampsia in the United Kingdom, it constituent countries and in the Regional Health Authorities of the English N.H.S.

Table 4.5. Incidence rate of eclampsia by maternal age and by multiple pregnancies.

Table 4.6. Country of birth of mother by live birth from eclamptic pregnancies in England and Wales and by all live births in England and Wales.

Table 4.7. Investigations carried out before and after first eclamptic seizure in hospitalised women.

Table 4.8. Maternal deaths associated with eclampsia in 1992 .............................. 85

Table 4.9. Major maternal complications recorded in hospital case-notes................ 86 
Table 4.10. Post eclamptic maternal morbidity recorded by general practitioners 86

Table 4.11. Morbidity in surviving infants recorded by special care baby units or the general practitioners.

Table 5.1. Differences in outcome between women with antenatal eclampsia who had a long interval ( $>14$ days) between tests on blood pressure and those women with antenatal eclampsia who had more frequent ( $\leq 14$ days) screening checks.

Table 5.2. Differences between women whose first fit was not preceded by proteinuric hypertension and those who did have established proteinuria and hypertension before their first fit.

Table 5.3. Differences between women greater than or equal to 35 years of age versus all other women.

Table 5.4. Differences between multiparous and primiparous women. 102

Table 5.5. Differences between postpartum eclampsia without pre-existing proteinuria and hypertension compared with all other eclampsia.

Table 5.6. Differences between Antepartum and Intra- \& Postpartum Eclampsia.

Table 5.7. Summary of differences in maternal and fetal outcome measures in women with very preterm, preterm and term eclampsia.

Table 5.8. Differences between preterm and term eclampsia.

Table 5.9. Variables, their type and coding system used in multiple logistic regression analyses.

Table 5.10. Multiple logistic regression model of variables independently associated with major maternal complications.

Table 5.11. Final multiple logistic regression model of variables associated with general practitioner reported maternal morbidity.

Table 5.12. Final multiple logistic regression model of variables associated with fetal/infant death.

Table 5.13. Final multiple logistic regression model of variables associated with infant morbidity. 115

\section{INDEX OF FIGURES}

Figure 1.1. A conventional model of the relationship between pre-eclampsia and eclampsia

Figure 1.2. The current explanation of the pathogenesis of the maternal syndrome of pre-eclampsia and eclampsia.

Figure 2.1. Pilot study: Outcome of notifications ................................................. 40

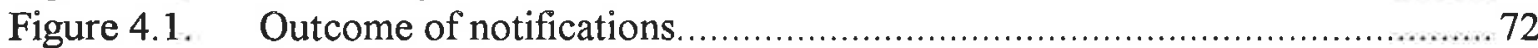

Figure 4.2. Completeness of data collection.................................................... 75

Figure 4.3. Graph showing the mean and standard deviation of the systolic and diastolic blood pressures at booking, immediately before seizure and the maximum recorded blood pressures.

Figure 4.4. Number of cases of eclampsia by gestational age at first seizure. 82 


\section{ABSTRACT}

Eclampsia, now rare in the United Kingdom, remains one of the major causes of maternal mortality in this country and world-wide. This is a prospective, descriptive study of every case of eclampsia that occurred in the United Kingdom during 1992.

All 279 hospitals with a consultant obstetric unit in the United Kingdom participated. Obstetricians and midwives notified 582 possible cases and 383 were confirmed as eclampsia. No additional cases were identified when checks were made with existing maternal data base systems. Detailed information about each case was collected from a hospital case-note review and a general practitioner questionnaire.

The national incidence of eclampsia was $4.9 / 10,000$ maternities ( $95 \%$ CI $4.50-5.4)$. In the majority of cases seizures occurred despite antenatal care (70\%) and within one week of the woman's last visit to a doctor or midwife (85\%). Seventy-five percent of first seizures occurred in hospital, and in $38 \%$ of these, before both proteinuria and hypertension had been documented. Women under 20 years of age or with multiple pregnancy were particularly susceptible. Most cases (44\%) occurred after delivery, more than a third (38\%) antepartum, and the remainder (18\%) intrapartum.

The maternal case fatality rate was nearly 1 in 50 women (1.8\%). Thirty-five percent of all women had at least one major complication and of those who survived $63 \%$ had some residual morbidity reported by their general practitioner. The stillbirth and neonatal mortality rates were $22.2 / 1000$ and $34.1 / 1000$ respectively.

Multiple logistic regression analyses were carried out to help assess which factors of the eclamptic episode were associated with poorer maternal and fetal outcomes. Antepartum seizures and high maternal blood pressures were associated with maternal complications, while the factor most strongly associated with fetal/infant death and infant morbidity was the occurrence of seizures preterm. 


\section{STATEMENT OF ORIGINALITY}

This work contains no material which has been accepted for the award of any other degree of diploma in any university or other tertiary institution and, to the best of my knowledge and belief, contains no material previously published or written by another person, except where due reference has been made in the text.

I give consent to this copy of my thesis, when deposited in the University Library, being available for loan and photocopying.

Signed

$16 / 6 / 95$

Kirsty A. Douglas

Date 


\section{ACKNOWLEDGEMENTS}

Throughout my time in Oxford I was supported financially by the Reginald Walker Fellowship from the University of Adelaide. The study was funded by the Medical Audit Unit of the Royal College of Obstetricians and Gynaecologists and would not have been possible without the co-operation of over 800 obstetricians and several hundred midwives and general practitioners throughout the United Kingdom. My grateful thanks are extended to them all.

Chris Redman provided excellent supervision for this work and I thank him for his wise counsel, exacting standards, the example of his phenomenal hard work and for the blessed relief of the occasional game of racing demon. Thanks also Caroline Crowther who had the difficult task of supervising a student who was in a different country but never-the-less gave her support unstintingly.

Without Iain Chalmer's advice and gentle lessons in diplomacy I would never have managed to persuade the Royal College of Obstetrics and Gynaecology (RCOG), the Royal College of Midwives (RCM) and the Royal College of General Practitioners (RCGP) to support the project. Adrian Grant provided invaluable practical advice particularly on how to maximise response rates. Alice McPharline was a useful resource person on national statistics and Lilia Duley was always a welcome ally. All four shared information willingly, spent time ungrudgingly and extended a warm welcome; characteristics that all at the National Perinatal Epidemiology Unit are renowned for.

Thanks to Mike Maresh and Marion Hall for helping to secure funding and political support for the project from the Medical Audit Unit of the RCOG.

Valuable advice and information was also received from: Dr David Jewel from the Association for GP Maternity Care, Richard Lynne of the British Paediatric Surveillance Unit, Elizabeth Cloake and Dr D Milner from the Confidential Enquiries into Maternal Deaths, David Canham of the Office of Population Census \& Statistics (OPCS), Jean Chapel and Anne Welch from the North-West Thames regional database, Dr E. Coles from the Cardiff Birth Survey, and Dr Susan Cole from the Directorate of Information Services, National Health Service in Scotland. Thanks also to Pat Yudkin and Robyn Attewell for statistical advice and assistance.

Helen Temple worked as a part-time secretary on the project and her enthusiasm, hard work, continued friendship and shared morning teas were greatly appreciated. 
The whole task would have been less enjoyable and less memorable without the laughter, tears and friendship of Davina Buckley and Pip Kyle with whom I shared an office for three years. The High Risk Pregnancy Unit in the Nuffield Department of Obstetrics and Gynaecology became a home away from home as well as providing more than the odd manhour folding notification forms and sticking envelopes; thank you to; Vebeke, Tansy, June, Jan, Jinetta, Karen, Vicente, Joe, Peter, Geraldine, Joy and several helpful (\& bored) patients.

Thank you also to Jane Ramsey, Tom Jaensch, and Lucie and Phil Walters who have been great mates and on several occasions were invited to dinner and were forced to "sing for their supper" by stuffing envelopes.

Many grateful thanks to my father, Professor Bob Douglas of National Centre for Epidemiology and Population Health who not only provided regular injections of enthusiasm and encouragement but also acted as a third and unofficial supervisor when it was most needed. Thanks also to Professor Jeffrey Robinson who was the first person to encourage my interest in obstetric medicine, was instrumental in helping me to get to Oxford and who has continued to act as a mentor over the last 4 years. 
This thesis is dedicated to my husband in loving appreciation of all he has done to make it possible. Without his enduring faith in me, his active participation in the work, his care of James and his enthusiasm for everything life has to offer it never would have been completed.

Thank you Mounty 


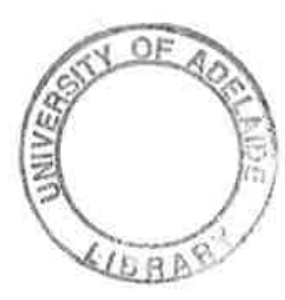

\section{CHAPTER 1.}

\section{LITERATURE REVIEW}


This thesis describes a descriptive study of all cases of eclampsia that occurred in the United Kingdom in 1992. This chapter is a brief review of the current knowledge of eclampsia and pre-eclampsia. It examines the need for a national study and formulates the prior hypotheses that were addressed by the study.

\subsection{FIRST DESCRIPTIONS- HISTORICAL PERSPECTIVE.}

Descriptions of generalised convulsions in pregnancy and the puerperium exist in literature from ancient Egypt and Greece (MacGillivray, 1983b). The term eclampsia is derived from a Latin compound verb meaning 'to flash' (Macintosh, 1952) and was first used in its current context by De Sauvages in 1739 (Chesley, 1980) to describe convulsions of acute causes. De Sauvages described several species of acute seizures including Eclampsia parturientium which were gestational convulsions attributable to irritation of the uterine cervix. Mauriceau in 1668 had written a clear description of such seizures but he and others before him failed to distinguish the cause from chronic epilepsy (Chesley, 1980).

When the condition was first described the pathophysiology of the disease was completely unknown and theories of causality were therefore dictated by awareness of symptoms and signs associated with the seizures. In 1843 Lever in London and Simpson in Edinburgh independently documented the association of oedema and proteinuria with eclamptic seizures (MacGillivray, 1983b) and the next 50 years were dominated by the theory that eclampsia was primarily a renal disease. In late nineteenth and early twentieth century the association of hypertension with eclamptic convulsions was noted by Vaquez and confirmed by Cook and Briggs (Chesley, 1978a) and this sparked a period where the syndrome was seen by some to be a latent form of chronic hypertension.

Work on eclampsia from the eighteenth to early twentieth centuries consisted of attempts to identify and understand signs and symptoms associated with eclampsia. By the mid twentieth century hypertension, proteinuria and oedema were firmly established as the traditional triad of signs on which the diagnosis of pre-eclampsia/eclampsia were based, but there was still no real understanding about the underlying pathophysiology of the condition.

A multitude of theories were developed about the cause of eclampsia including cerebral anaemia, cerebral congestion, ureteric obstruction and uterine stimulation. The concept of eclampsia as a toxaemia was first discussed as early as 1849 by Smith and by 1901 the theory was widely accepted (Chesley, 1976). Others persisted in the view that preeclampsia/eclampsia was a manifestation of chronic process (i.e. chronic renal disease or chronic hypertension) that was somehow altered by pregnancy rather than a condition 
specific to pregnancy. In 1905 DeLee reviewed the literature and concluded "We know practically nothing of the causation of eclampsia" (Loudon, 1991).

\subsection{CLASSIFICATION AND DEFINITIONS OF PRE-ECLAMPSIA AND ECLAMPSIA.}

\subsubsection{Classification system.}

Classification of pre-eclampsia and eclampsia has been a subject of continuing debate. The American Committee on Maternal Welfare developed the first US classification system in 1940 (Bell et al. 1940) and it was refined and changed in 1952 (Eastman et al. 1952), 1972 (Hughes, 1972), 1986 (American College of Obstetricians and Gynecologists, 1986) and 1990 (National High Blood Pressure Education Program Working Group, 1990). Different systems have applied in England, Germany and Switzerland (MacGillivray, 1983b) and French authors had used 5 different classification systems by 1968 (Davies, 1971).

International variations in classification systems persist but the one shown below in Table 1.1. is widely used and has been accepted in principle by the International Society for the Study of Hypertension in Pregnancy, and the World Health Organisation (Davey \& MacGillivray, 1988).

\subsubsection{Definition of pre-eclampsia.}

Using the classification system in Table 1.1. pre-eclampsia is then the development of hypertension and proteinuria during pregnancy, labour, or in the puerperium in a previously normotensive non proteinuric woman. However it is far from being this simple: at the same time as debate on how to classify the diseases there has been continued vigorous debate about what absolute levels of blood pressure, proteinuria and oedema should be considered significant.

The level of blood pressure considered to be diagnostic of hypertension has varied considerably. In 1933 Browne (1933) suggested that any levels greater than $130 \mathrm{~mm} \mathrm{Hg}$ systolic or $70 \mathrm{~mm} \mathrm{Hg}$ diastolic were abnormal. That level is now considered too low and more recently, measurements of blood pressure at or above $140 \mathrm{~mm} \mathrm{Hg}$ systolic or $90 \mathrm{~mm}$ $\mathrm{Hg}$ diastolic have been diagnostic of hypertension although there have been numerous minor variations in this. Some authors indicate that the incremental change from a first trimester or pre-pregnancy level is important (Redman \& Jefferies, 1988; National High Blood Pressure Education Program Working Group, 1990); others insist that there must be two consecutive High readings a prescribed number of hours apart (Davey \& MacGillivray, 
1988; Australian Society for the Study of Hypertension in Pregnancy, 1993). There has also been debate as to whether phases 4 or 5 of the Korotkoff sounds should be used to determine the diastolic level and the correct position of the woman when the blood pressure is measured.

TABLE 1.1. Classification of hypertensive disorders of pregnancy.

\section{A. Gestational hypertension and/or proteinuria.}

Hypertension and/or proteinuria developing during pregnancy, labour, or in the puerperium in a previously normotensive non proteinuric woman.

1. Gestational hypertension (without proteinuria)

2. Gestational proteinuria (without hypertension)

3. Gestational proteinuric hypertension (pre-eclampsia)

B. Chronic hypertension and chronic renal disease.

Hypertension and/or proteinuria in pregnancy in a woman with chronic hypertension or chronic renal disease diagnosed before, during, or after pregnancy subdivided into;

1. Chronic hypertension (without proteinuria)

2. Chronic renal disease (proteinuria with or without hypertension)

3. Chronic hypertension with super-imposed pre-eclampsia

C. Unclassified hypertension and/or proteinuria.

Hypertension and /or proteinuria found either i. At first examination after twentieth week of pregnancy in a woman without known chronic hypertension or chronic renal disease, or ii. During pregnancy, labour, or the puerperium where information is insufficient to permit classification subdivided into

1. Unclassified hypertension (without proteinuria)

2. Unclassified proteinuria (without hypertension)

3. Unclassified proteinuric hypertension

\section{Eclampsia.}

The occurrence of generalised convulsions during pregnancy, during labour, or within 7 days of delivery and not caused by epilepsy or other convulsive disorders.

The definition of proteinuria has been less contentious. Most authors consider $0.3 \mathrm{gm}$ per litre or more of protein in a 24 hour sample is significant. As 24 hour samples are not always available alternative criteria using dipstick testing have been offered: either 1+ (National High Blood Pressure Education Program Working Group, 1990) or 2+ (Redman \& Jefferies, 1988). 
The importance of oedema has been gradually downgraded. It was recognised that oedema occurred in many normal pregnancies and is difficult to quantify objectively. Oedema is still recognised as part of the pre-eclampsia syndrome but its presence or absence no longer holds diagnostic significance.

Hypertension and proteinuria form the backbone for the diagnosis of pre-eclampsia but there has been increasing recognition that it is a multi-system disorder and there are now other biochemical changes which are seen to be significant, if not diagnostic, of preeclampsia. These include elevations in serum creatinine, urate (Redman \& Bonnar, 1978), bilirubin or transaminases (Weinstein, 1982), thrombocytopenia or evidence of haemolysis or disseminated intravascular coagulation (Perry, Jr. \& Martin, 1992).

\subsubsection{Definition of eclampsia.}

Eclampsia is defined as the presence of generalised seizures in a woman with pre-eclampsia that cannot be attributed to other causes (National High Blood Pressure Education Program Working Group, 1990). This is the most widely accepted definition and is adopted throughout this thesis. However, as with pre-eclampsia, the definition of eclampsia is not straightforward. Some authors particularly from North America identify eclampsia in women with pre-eclampsia and coma irrespective of whether or not there are seizures (Sibai, 1988; Roberts, 1989). A few neurologists have argued that any central nervous symptomatology in the context of pre-eclampsia should be termed eclampsia (Donaldson, 1989; Trommer et al. 1988; Raroque, 1989). Neither of these criteria has been widely accepted by the obstetric community (Goodlin, 1988).

\subsection{THE EPIDEMIOLOGY OF ECLAMPSIA.}

Epidemiology is the study of the distribution and determinants of disease frequency in human populations (Hennekens \& Buring. 1987). Properly conducted epidemiological studies can provide important clues to the biological basis of the disease. They require "systematic and accurate observation and recording of clearly defined facts in a total population or a representative sample" (Baird, 1977).

\subsubsection{The difficulties in studying the epidemiology of eclampsia.}

Unfortunately some researchers of eclampsia and pre-eclampsia still fail to make their definitions of these conditions explicit (Bhose, 1964; Sumnulu et al. 1989). The problem is compounded if the results of different studies are compared as it becomes impossible to be sure that the studies are looking at the same disease process. Davies (1971) in his review of 
the geographical epidemiology of the toxaemias of pregnancy concludes that it is impossible to make "any international comparison even of the grossest sort".

Many studies of eclampsia are case series collected in large, specialised centres (Lopez Llera, 1982; Adetoro, 1990; Sibai, 1990a). The populations treated at the centres are often not representative of the larger population so that only limited inferences can be drawn from the studies.

Studies in developing countries pose special difficulties such as inaccurate or non existent basic population statistics, a high rate of women who never come into contact with medical care even after severe complications and restricted resources with which to carry out research. In the developed world difficulties arise because the eclampsia is now rare, so to collect a large number of cases from one centre, studies extend over a very long time span (Pritchard \& Pritchard, 1975; Sibai, 1990a). Studies carried out over 10 - 20 years may well include cases managed under different management protocols. Combining all these cases together may hide the significance of these changes and thus weaken the value of the study.

These considerations make comparisons between studies in pre-eclampsia and eclampsia difficult and the following review should be read with these limitations in mind.

\subsubsection{Incidence.}

Very few studies of eclampsia have been done in total or even representative populations. Incidence rates quoted in studies must therefore be viewed with caution.

\subsubsection{Developed world.}

In 1922 Eden stated that the incidence of eclampsia in hospital populations of Britain was 8/1000 labours and estimated that the incidence in the general population would be considerably lower (Eden, 1922). Population based studies in Scotland (during 1965-77) and Wales (during 1965-74) found rates of $0.74 / 1000$ and $0.72 / 1000$ respectively (Templeton \& Campbell, 1979; Wightman et al. 1978). Other more recent population based studies come from Sweden with an incidence of $0.29 / 1000$ for 1976-80 (Moller \& Lindmark, 1986) and the USA incidence $0.56 / 1000$ during 1979-86 (Saftlas et al. 1990).

An example of the differences between hospital and population based studies is shown by comparing the population study of Saftlas referred to above with any of the USA hospital based studies which have found incidence rates varying from 1.3/1000 in 1975 (Pritchard \& Pritchard, 1975) to 3/1000 in 1990 (Sibai, 1990a). 


\subsubsection{Developing world.}

Even fewer population based studies have been done in the developing world and almost the only information on incidence rates of eclampsia comes from hospital based studies. In the developing world a far greater proportion of women deliver in the community and so the hospital populations are particularly skewed.

Doll and Hanington carried out an international survey of eclampsia in 1958-59 and found many countries had an estimated incidence in the range of 1.2-2.6/1000 deliveries (Doll \& Hanington, 1961), however their study included only primigravidae admitted to hospitals.

More recent studies done in a single hospital in a developing country have found incidence rates that are still in this range: South Africa 2.3/1000 (Moodley et al. 1983), Zambia 2.2/1000 (Chatterjee et al. 1978), Thailand 2.1/1000 (Porapakkham, 1979). However other studies report much higher rates (India 14.4/1000 (Raman \& Venkatesh, 1982), Nigeria 10.2/1000 (Agobe et al. 1981), Malaysia 6.6/1000 (Nalliah \& Abdullah, 1990) South Africa 4.8/1000 (Moore \& Munoz, 1985)) or much lower rates (Zimbabwe 0.6/1000 (Crowther, 1985a), Hong Kong 0.8/1000 (Liang et al. 1984) and India 0.64/1000 (Dhall, 1984)).

It would be inaccurate to extrapolate an incidence rate in one hospital to the whole country. Even in the same country the rates may vary during similar periods, for example from 10.2/1000 to 4.2/1000 deliveries in Nigeria (Agobe et al. 1981; Adetoro, 1990) and from $14.4 / 1000$ to $0.64 / 1000$ in India (Raman \& Venkatesh, 1982; Dhall, 1984). It seems likely that in poorer countries hospital series will overestimate the incidence in the population as a biased sample of complicated pregnancies will be delivered in hospital.

One of the few total population studies was carried out by Davies in Jerusalem and he found an incidence of eclampsia of 0.6/1000 (Davies et al. 1970) in the years 1964-66. In Cali, Colombo between 1964-70 Neutra found an incidence of eclampsia of 1.59/1000 (Neutra, 1975) which he believed was a true reflection of the incidence in the general population.

\subsubsection{Change over time.}

In the developed world figures suggest that there may have been a substantial decrease in the incidence of eclampsia from the first to the second half of this century. However, New Zealand is the only country where the evidence is reliable because for many years eclampsia was a notifiable condition and continuous good records of incidence were kept. In New Zealand the incidence of eclampsia declined from 3.2/1000 to $0.8 / 1000$ between $1928-33$ and 1956-58 (Corkill, 1961). Explanations for this decrease included the implementation of 
routine antenatal screening or general improvements in the health and nutritional status of pregnant women.

There is no good evidence from which it is possible to draw any conclusions about changes in incidence rates over time in the developing world. Certainly in many developing countries antenatal care is still rare and nutritional status of pregnant women less than optimal.

\subsubsection{Maternal Mortality.}

Maternal mortality rates in the industrialised societies are in the range of 5-15 per 100,000 live births. The disparity between the developed and developing countries is huge; in many areas of the developing world the maternal mortality rates are in the range of 200-1000 per 100,000 live births (Rosenfield, 1992).

Despite these differences in overall mortality rates the proportion of all maternal deaths attributable to eclampsia seems to be similar in both developed and developing countries. Duley (1992a) reviewed maternal mortality associated with hypertensive disorders of pregnancy in the developing world and found that $10 \%$ of all maternal deaths are associated with eclampsia. Evidence from the Confidential Enquiries into Maternal Deaths in the United Kingdom indicates that deaths from hypertensive disorders of pregnancy account for $15-20 \%$ of all deaths and eclampsia for about $7.5-10 \%$ (DHSS et al. 1991).

\subsubsection{Developed world.}

Case series from industrialised countries have found case fatality rates varying from $0 \%$ (Wightman et al. 1978) to 5.8\% (Gedekoh et al. 1981). The population based studies have shown case fatality rates of eclampsia of $0 \%$ (Templeton \& Campbell, 1979), 0.8\% (Medical Research Council of New Zealand, 1962) and 1.2\% (Moller \& Lindmark, 1986). However many of these studies involve relatively small numbers of cases and so that one maternal death can make a large difference in case fatality rate.

\subsubsection{Developing world.}

Unsurprisingly, the case series from developing nations show considerably higher case fatality rates than those from developed nations. The rates vary from $5 \%$ (Nalliah \& Abdullah, 1990; Porapakkham, 1979) to 16\% (Chatterjee et al. 1978) although some have reported rates as low as 2\% (Mphahlele, 1975). One of the few studies from the third world which purports to be representative of the population found a case fatality rate of $7.7 \%$ in Cali Colombo (Neutra, 1975). 


\subsubsection{Change over time.}

In the United Kingdom the overall maternal mortality rate per 100,000 total births has decreased from 67.1 in 1955-7 to 7.6 in 1985-7 (DHSS et al. 1991). In 1922 Eden estimated a case fatality rate of eclampsia of $22.5 \%$ whereas in the 1960 's and 70 's the eclampsia case fatality rates in two small UK population based studies were $0 \%$ (Templeton \& Campbell, 1979; Wightman et al. 1978). Similar dramatic decreases have been documented in Sweden where the crude maternal mortality rates dropped from 350/100,000 live births in $1930-5$ to $5 / 100,000$ live births in 1976-80 (Hogberg \& Joelsson, 1985). The case fatality rate for eclampsia in Sweden fell from $13.9 \%$ in $1951-5$ to $3.1 \%$ in $1971-80$. The decreases have been attributed to advances in medical care as well as socio-economic improvements (Hogberg \& Joelsson, 1985).

There is no evidence to suggest that the risk of dying from hypertensive disorders of pregnancy in Africa or in Latin America is decreasing (Duley, 1992a). This is probably attributable to the quality and accessibility of health services in these countries.

\subsubsection{Maternal Morbidity.}

Measurements of rates of maternal morbidity are likely to be even less reliable. Almost all the studies are hospital based, not representative and those conducted in the developed world include only a small numbers. Often only major morbidity is documented if any at all. Comparisons between studies are difficult because definitions of morbidity vary or are not clearly stated. No studies have attempted to document minor morbidity by follow-up of the women once they have returned to the community.

\subsubsection{Developed world.}

Templeton's population based study from the Grampian region in Scotland found $11 \%$ of women had "serious complications" (Templeton \& Campbell, 1979). This is lower than, but in the same range as the evidence from Sweden where $15 \%$ of all women with eclampsia developed major complications (Moller \& Lindmark, 1986).

\subsubsection{Developing world.}

There are no good population based studies from the developing world which give any information on the rates of maternal morbidity. However case series from Mexico City and two different centres in Nigeria report overall rates of maternal morbidity of $23 \%, 31 \%$ and $21 \%$ respectively (Lopez Llera, 1982; Konje et al. 1992; Adetoro, 1990). In terms of specific complications case series report acute renal failure in 2-4\% (Adetoro, 1990; Moore 
\& Munoz, 1985; Nalliah \& Abdullah, 1990; Moodley et al. 1983), pulmonary oedema in 15\% (Porapakkham, 1979; Nalliah \& Abdullah, 1990), placental abruption in 1-8\% (Porapakkham, 1979; Moodley et al. 1983; Nalliah \& Abdullah, 1990) and puerperal psychosis of between 1 and 3\% (Miles, Jr. et al. 1990; Moodley et al. 1983)

\subsubsection{Perinatal Mortality.}

In the first half of the century Eden (1922) estimated that infant mortality rates in eclampsia were between $34 \%$ and $54 \%$ in the United Kingdom and a large proportion of these were in preterm infants. Eden concluded that "the infantile mortality of eclampsia is very high and must inevitably remain so" (Eden, 1922). He was wrong; advances in perinatology (particularly in the care of preterm infants) in the last 70 years have led to striking changes in the overall perinatal mortality rates and this has been reflected in decreases in the perinatal mortality rates associated with eclampsia.

\subsubsection{Developed world.}

In two population based studies conducted in 1965-77 and 1965-74 in the United Kingdom the perinatal mortality rate associated with eclampsia varied from $11 \%$ to $21 \%$ respectively (Templeton \& Campbell, 1979; Wightman et al. 1978). Neither the Swedish or USA population based studies of eclampsia give overall perinatal mortality rates. Case series from the developed world have perinatal mortality rates varying from $9 \%$ to $26 \%$ (Sibai et al. 1981; Gedekoh et al. 1981). A consistent feature in all studies and series is a high proportion of fetal loss associated with prematurity and small for gestational age infants.

\subsubsection{Developing world.}

Once again in the developing world there is a complete lack of reliable information on perinatal mortality rates associated with eclampsia. Perinatal mortality rates that are quoted from surveys and case series range from 3\% (Liang et al. 1984) to 60\% (Sumnulu et al. 1989) although most are in the range of 18\%-30\% (Moodley et al. 1983; Konje et al. 1992; Neutra, 1975; Lopez Llera, 1982; Crowther, 1985b)

\subsubsection{Risk factors associated with pre-eclampsia and eclampsia.}

Many different risk factors have been associated with pre-eclampsia and eclampsia. These can be roughly divided into maternal, fetal and environmental and are shown in Table 1.2. 
TABLE 1.2. Risk factors for pre-eclampsia and eclampsia.

\begin{tabular}{|c|}
\hline Maternal risk factors \\
Parity \\
Age \\
Race \\
Genetic factors \\
Body build \\
Cigarette smoking \\
Others \\
Fetal risk factors \\
Multiple pregnancy \\
Fetal sex \\
Fetal abnormalities \\
Environmental risk factors \\
Diet \\
Provision of antenatal care \\
Socio-economic status \\
Weather \& seasonal variations \\
\hline
\end{tabular}

\subsubsection{Maternal risk factors.}

\section{Parity.}

It was recognised by Mauriceau in the seventeenth century that women in their first pregnancy were particularly prone to eclampsia and more recently the same has been observed in pre-eclampsia. The high proportion of primigravidae in series of eclampsia is a remarkably consistent finding in studies of eclampsia as shown in the Table 1.3. below.

TABLE 1.3. Proportion of primigravidae in published series of eclampsia.

\begin{tabular}{lccc}
\hline $\begin{array}{c}\text { Name of first } \\
\text { author }\end{array}$ & $\begin{array}{c}\text { Year of } \\
\text { publication }\end{array}$ & $\begin{array}{c}\text { Country study } \\
\text { condlucted in }\end{array}$ & $\begin{array}{c}\text { Proportion of } \\
\text { primiparae }\end{array}$ \\
\hline Eden & 1922 & United Kingdom & $69 \%$ \\
Pritchard & 1975 & USA & $85 \%$ \\
Wightman & 1978 & Wales & $74 \%{ }^{*}$ \\
Templeton & 1979 & Scotland & $77 \% *$ \\
Porapakkham & 1979 & Thailand & $65 \%$ \\
Llang & 1984 & Hong Kong & $91 \%$ \\
Moller & 1986 & Sweden & $70 \%$ * \\
Sibai & 1986 & USA & $85 \%$ \\
\hline \multicolumn{2}{c}{ *Population based series }
\end{tabular}

The degree of increased relative risk of primigravidae versus multigravidae is not as uniformly consistent probably because of the different populations the studies are drawn 
from. Primigravidae are said to have an incidence rate that is 3.5 (Porapakkham, 1979), 6 (Neutra, 1973; Adetoro, 1990) or 7 (Liang et al. 1984) times the rate for multigravidae.

Some have noted an increased risk of pre-eclampsia and eclampsia amongst grand multiparae (Porapakkham, 1979; Moore \& Munoz, 1985). This finding is not universal and the increased risk may be more a factor of the woman's age than of her grand multiparity.

Age.

Increasing age over 30 appears to be one of the important factors affecting incidence of preeclampsia (Davies, 1971). Many of the population based studies of pre-eclampsia describe a $\mathrm{J}$ shaped curve of incidence; with moderately high incidence in very young women, decreased incidence between the age of 20 and 35 and then markedly increased incidence in the over 35's (Hauch \& Lehmann, 1934; Rauramo, 1961; Davies et al. 1976).

Recent population based studies have shown markedly increased incidence of eclampsia in women under the age of 20 (Saftlas et al. 1990) and in women over the age of 40 (Moller \& Lindmark, 1986).

Age and parity often go together and in his review of the geographical epidemiology of the toxaemias of pregnancy Davies (1971) concluded that primiparae show a higher rate of eclampsia than multiparae at all ages and that elderly primiparae are at a particularly high risk of pre-eclampsia and eclampsia.

\section{$\underline{\text { Race. }}$}

The question of whether race affects the incidence of pre-eclampsia or eclampsia is complex because differences in geographical and socio-economic factors between racial groups confound the issue.

In series from North America black women have higher incidence of pre-eclampsia and eclampsia however this may only be a reflection of higher incidence of chronic hypertension or poorer antenatal care (MacGillivray, 1983c). Davies' population based study did find a significantly higher incidence of pre-eclampsia in Muslim Arabs than Jews born in North Africa and Israel (Davies et al. 1970). There may well be racial differences in the incidence of pre-eclampsia and eclampsia but the evidence is not conclusive. 


\section{Genetic factors.}

There is a familial tendency in susceptibility to pre-eclampsia and eclampsia. Chesley et al (1968) found that daughters of women with eclampsia had a $26 \%$ rate of pre-eclampsia compared to $8 \%$ of the daughter-in-laws. Likewise a higher incidence of pre-eclampsia was found in the mothers but not mother-in laws of pre-eclamptic women (Sutherland et al. 1981). More recently, several workers have tried to explain the familial tendency on a genetic basis using various gene models (Cooper et al. 1988; Chesley \& Cooper, 1986; Liston \& Kilpatrick, 1991). Homozygosity of a single recessive gene conferring susceptibility to pre-eclampsia seems the most likely but the evidence is not conclusive.

\section{Body build.}

There has been controversy over whether or not weight or height affect the incidence of pre-eclampsia or eclampsia (Davies, 1971). Once again differences in classifications may have affected the conclusions; for example some of the earlier studies may have included women with chronic hypertension (which is known to be related to obesity) rather than true pre-eclampsia.

More recently Chesley concluded "eclampsia spares no habitus, but has a slight predilection for underweight women" (Chesley, 1984). While studying risk factors in the USA Eskenazi et al (1991) found that, regardless of parity, women with a high body mass index had a greater risk of developing pre-eclampsia. In summary there is no conclusive evidence that either high or low body mass affects the risk of women developing pre-eclampsia.

\section{Cigarette smoking.}

As early as the late 1960 's there was evidence from Aberdeen that women who smoked had a significantly lower incidence of pre-eclampsia than women who did not. It was found that this was independent of social class (Duffus \& MacGillivray, 1968; Butler \& Alberman, 1969). In a more recent case control study Marcoux et al (1989a) confirmed that smoking in early pregnancy reduced the risk of developing pre-eclampsia, the relative risk of preeclampsia decreasing with an increasing number of cigarettes smoked. However the harmful effects of smoking on pregnancy outcome (i.e. particularly in relation to retarded intrauterine fetal growth) outweighed the protective effect against pre-eclampsia.

\section{Others.}

A number of other maternal medical conditions are associated with increased risk of developing pre-eclampsia. It is now generally accepted that chronic hypertension, diabetes, 
and collagen vascular diseases are associated with increased risk of this condition (Nelson, 1955b; Roberts et al. 1989). A history of migraine (Marcoux et al. 1992) or working during pregnancy (Eskenazi et al. 1991) may also increase risk, whilst physically active leisure time may decrease risk (Marcoux et al. 1989b). Recent studies have failed to support the suggestions that use of the oral contraceptives (Bracken \& Srisuphan, 1982) or barrier contraceptive methods (Mills et al. 1991) increases risk of pre-eclampsia.

\subsubsection{Fetal risk factors.}

\section{Multiple pregnancy.}

The incidence of spontaneously conceived twins in the general population is around $1.2 \%$. In case series, women with multiple pregnancies account for between $3 \%$ and $13 \%$ of the eclamptic population (Liang et al. 1984; Moller \& Lindmark, 1986; Wightman et al. 1978; Eden, 1922; Konje et al. 1992). The increased risk of pre-eclampsia in twin pregnancies is now well accepted and has been estimated at between 3 (Lopez Llera et al. 1989) and 5 (MacGillivray, 1958) times that of singletons. There is still debate whether monozygosity or dizygosity of twins confers any extra risk (McFarlane \& Scott, 1976; Stevenson et al. 1976).

\section{Fetal sex.}

Since the beginning of the century there have been intermittent reports of hospital based studies which indicate that eclampsia (but not necessarily pre-eclampsia) is more frequent in women giving birth to a male child. MacGillivray (1983c) reviewed the literature prior to 1983 and concluded "although it is still possible that the male:female sex ratio is higher in cases of eclampsia, this is not true of proteinuric pre-eclampsia". Since this several further studies have been published. Campbell's study of a total population came to the same conclusion as MacGillivray i.e. that proteinuric pre-eclampsia was not associated with fetal sex (Campbell et al. 1983).

The evidence in relation to eclampsia continues to be inconclusive. Lopez Llera found a significant increase in the male:female sex ratio in antepartum eclampsia but not in postpartum eclampsia (Lopez Llera, 1990). The fascination with the concept of a sex ratio difference is based on the idea that eclampsia may be more common if the mother is carrying a foetus which is genetically more different. 


\section{Fetal abnormalities.}

There have been reports of increased incidence of pre-eclampsia in association with hydrops fetalis (Jeffcoate \& Scott, 1959) and hydatidiform mole (Newman \& Eddy, 1988). Nelson's population based study showed a lower incidence of fetal malformations in the preeclamptic population compared with the general population (Nelson, 1955a). However more recent studies have reported increased rates with trisomy 13 (Boyd et al. 1987; Swartjes et al. 1992) and triploidy (Paterson et al. 1971).

\subsubsection{Environmental risk factors.}

Diet.

There is an extensive literature on the effects of diet and nutrition on the incidence of preeclampsia and eclampsia. The rigour of some studies is questionable and the reports are often written in an emotive fashion. In 1965 the World Health Organisation stated that there was "no scientific basis for believing that either deficiency or excess of any essential nutrient predisposes to pre-eclampsia and eclampsia" (MacGillivray, 1983c).

Whereas previously it was believed that dietary factors cause pre-eclampsia now it is the converse, that dietary interventions can help prevent the condition. Belizan et al (1988) noted that the incidence of hypertensive disorders of pregnancy is low in some populations with a high calcium intake and prompted interest in calcium supplementation as a form of prevention. More recently Secher proposed fish-oil supplementation as a preventive measure for pre-eclampsia and has describe a possible biochemical mechanism for its action (Olsen \& Secher, 1990). He has successfully initiated a multi-centre controlled trial of fishoil supplementation.

\section{Provision of antenatal care.}

There is some good evidence that the reduction in incidence of eclampsia in the developed world over the last century is directly related to the introduction of antenatal care (Corkill, 1961 ; Hogberg \& Joelsson, 1985). The relative lack of care is also commonly described as a reason for the continued high rates of eclampsia in the developing world. In his review Davies stated that there "is overwhelming evidence of the value of antenatal care in preventing severe pre-eclampsia and, especially eclampsia" (Davies, 1971).

Underlying these statements is a conviction that once pre-eclampsia is diagnosed then intervention can prevent it becoming severe or "progressing" to eclampsia. There have been no studies which demonstrate that antenatal care per se prevents pre-eclampsia. Indeed it 
has to be recognised that antenatal care is not a panacea; since cases of eclampsia occur unexpectedly (Eden, 1922; Crowther, 1985b; Liang et al. 1984) and an increasing number of studies describe "unpreventable" eclampsia (Sibai et al. 1986a; Campbell \& Templeton, 1980).

\section{$\underline{\text { Socio-economic status. }}$}

The question whether or not pre-eclampsia and eclampsia are more common in women of a particular socio-economic group is complex.

There have been several reports which suggest that eclampsia is more common in women from lower socio-economic groups and some have also suggested that the same is true for pre-eclampsia. If this is so it is still difficult to try to interpret why as there are many potential confounding factors. The effect could simply be one of age and parity as there are likely to be a higher proportion of very young primiparous women and older highly parous women in the lower socio-economic groups. Alternatively there may be differences in the antenatal care that women from these groups receive, or differences in diet that confer susceptibility to pre-eclampsia and eclampsia. The difficulties in interpretation are made worse because of the paucity of population based information.

Population based studies in the Aberdeen area have shown no (Davies et al. 1970) or very little difference (Duffus \& MacGillivray, 1968; MacGillivray, 1983c) in the incidence of eclampsia in women from different social classes. In contrast to those findings is that of Davies' study in Jerusalem which was conducted in a total community where he felt all members had equal access to standard medical care. He used educational level as an index of socio-economic status and stratified his analysis to take into account age, parity and ethnicity. He found that the pre-eclampsia rate in illiterate (poorest) women was twice that of the average (Davies et al. 1970).

\section{Weather and seasonal variations.}

Several studies purport to find a correlation between weather patterns and the incidence of pre-eclampsia or eclampsia. Reviewers of this literature have criticised the methodology used and been sceptical about the significance of their findings (Davies, 1971; MacGillivray, 1983c) particularly as different studies have correlated dry, humid, cool, hot or changes in weather with increased incidence. However interest in the subject has continued. Neutra conducted a population based study and controlled for variations in the number of births, primiparae and very young women and still found that eclampsia rate were twice as high on cool or humid days than on days with average temperature (Neutra, 1974). In contrast a 
population based case-control study in the US found no evidence to support an association between eclampsia and weather (Alderman et al. 1988). In summary, the case for such an association remains unproved.

\subsection{PATHOGENESIS OF PRE-ECLAMPSIA AND ECLAMPSIA.}

The pathogenesis of pre-eclampsia and eclampsia is still incompletely understood. Our understanding has been delayed by overemphasis on the symptoms that define the syndrome. For example, when the association with hypertension was noted attempts were made to explain the condition in the context of theories of hypertension as if hypertension was the cause rather than one of the effects of the syndrome. Recently progress has been made by going back to some of the most basic and earliest observations about the patterns of disease and by looking for a unifying concept which can explain the diverse signs and symptoms of the syndrome.

\subsubsection{Pre-eclampsia.}

The syndrome of pre-eclampsia occurs only in fetal (uterine or abdominal) or molar pregnancies. This suggests that the origin of the condition lies with the placenta which is the only organ unique to both. Page (1948) was one of the first authors to propose placental ischaemia as a "precipitating cause of pre-eclampsia". More recent work has provided histological and epidemiological support for his theory.

\subsubsection{The placenta - the origin.}

Placentas of pre-eclamptic women show changes in the structure of the spiral arteries. There is limited trophoblastic invasion into the walls of the spiral arteries (Redman, 1991). These changes occur as early as 8-18 weeks gestation and predispose to actual or relative placental ischaemia by limiting the physiological dilatation of the arteries.

The role of placental ischaemia is supported by the higher incidence of pre-eclampsia in conditions with increased placental mass (twins, hydrops fetalis, hydatidiform mole) and medical conditions which involve an element of microvascular disease (diabetes, chronic hypertension, collagen vascular disease) (Roberts et al. 1989). It is also reinforced by the fact that many of the fetal effects of the pre-eclampsia syndrome (i.e. fetal intrauterine growth retardation and fetal hypoxia) are consistent with placental ischaemia (Lopez Llera et al. 1972). 


\subsubsection{Endothelial cell damage - the initiator.}

The signs and symptoms of pre-eclampsia are diverse but can all be explained as a consequence of damage to vascular endothelial cells. The endothelial cell lining of the vasculature is a monocellular contiguous layer which forms the interface between the intravascular and extravascular compartments (Friedman et al. 1991). The lining normally has vasodilatory and anticoagulant properties mediated by a number of factors including Protein C (anticoagulant) and prostacyclin (potent vasodilator) (Roberts \& Redman, 1993). When the cell lining is damaged there is not only a loss of the normal functions but also the surface expression of procoagulants which stimulate platelet aggregation, activation of the clotting cascade, clot formation and the production of vasoconstrictor substances (Roberts \& Redman, 1993). This results in increased vessel permeability, clot formation and vasospasm thus increasing ischaemia further down the vessel pathway which, in turn increases damage to distal endothelial cells and thus exacerbates the original disturbance.

Hypertension in pre-eclampsia is thought to be caused by altered endothelial control of vascular tone with both an excess of endothelial vasoconstriction factors and a deficit of vasodilators. Proteinuria can be explained as secondary to increased vascular permeability in the glomeruli of the kidneys and a primary endothelial cell lesion, glomerular endotheliosis, is a well established feature of pre-eclampsia. Oedema results from changes in the function of the endothelial cell layer as a interface between intra and extravascular compartments. Other disturbances such a low platelet count and coagulation disturbances may be a direct consequence of endothelial activation of the clotting system. The damage seen in kidneys, livers and brain in pathological examination of women with pre-eclampsia suggest that there has been ischaemia leading to haemorrhage and necrosis rather than massive vascular disruption. Thus it can be seen that damage to the vascular endothelial cells could be the mediator of all of these signs and symptoms of pre-eclampsia.

\subsubsection{The link between the placenta and endothelial cell damage.}

The link between the ischaemic placenta (the origin of the disease) and vascular endothelial cell damage (the mediator of symptoms) is the area where our knowledge of the pathogenesis of pre-eclampsia is most incomplete. It has been hypothesised that the ischaemic placenta releases or produces a blood borne product(s) into the maternal circulation which causes the endothelial cell damage but this is unproved. (Roberts \& Redman, 1993). 


\subsubsection{Eclampsia.}

Cerebral oedema, infarction, haemorrhage and transient vasospasm (Sibai, 1988) along with disseminated intravascular coagulopathy, and hypertensive or metabolic encephalopathy (Peterkin et al. 1992) have all, in the past, been implicated in the pathogenesis of eclamptic seizures. To try to shed light on the subject electroencephalograms (EEG's), computerised axial tomography scans (CT scans), magnetic resonance imaging (MRI) and angiography have been done in surviving women soon after their seizures as well as histopathological examination of the brains of women who have died following eclampsia.

The EEG's of women with eclampsia are almost always abnormal after an eclamptic seizure (Sibai et al. 1985; Pritchard et al. 1984; Macintosh, 1952) but there are no pathognomonic findings. and the abnormalities usually resolve within months (Sibai et al. 1985). CT scans are variable depending on the sophistication of the equipment used and the time delay between seizure and scan (Brown et al. 1988; Peterkin et al. 1992). Recent studies done immediately following seizures show reversible changes including areas of localised hypodensity which may represent oedema, small-vessel thrombosis, ischaemic softening or petechial cortical haemorrhages (Milliez et al. 1990; Dunn et al. 1986; Colosimo, Jr. et al. 1985). Magnetic resonance imaging has also shown reversible lesions which are thought to represent oedema in response to local ischaemic insult (Crawford et al. 1987; Horn et al. 1990). The changes are in the cortex, concentrated in the parieto-occipital regions (Raps et al. 1993; Sanders et al. 1991). Cerebral angiography has been performed in a number of cases of eclampsia and pre-eclampsia and have shown diffuse vasospasm of the large and middle sized intracranial vessels. (Trommer et al. 1988; Will \& Lewis, 1987) which lends support to the theory that vasoconstriction causes ischaemic damage. Recently there have been reports of eclamptic women with documented cerebral vasospasm, being treated with nimodipine, a specific cerebral vasodilator, with resolution of symptoms (Horn et al. 1990) and or vasospasm (Belfort et al. 1993).

Sheehan and Lynch (1973) conducted one of the most complete pathological studies on the brains of women who died after eclampsia. They found multiple petechial haemorrhages and microinfarctions in the cortex as had previously been described by Goven (1961). Other pathological changes have included subcortical haemorrhages of varying size, haemorrhages in the corona radiata, caudate nucleus or brain stem, cerebral oedema and small subarachnoid haemorrhages (Donaldson, 1989).

The distinction between eclampsia and pre-eclampsia depends upon the occurrence of generalised seizures. However, some women with pre-eclampsia have other cerebral 
pathology including retinal detachment, cortical blindness, hemiparesis, and coma without developing seizures. One of the difficulties in assessing the investigations is to differentiate between the changes which are the initiators of seizure activity and changes which give rise to other neurological symptoms of pre-eclampsia. It seems all of the pathology mentioned above are different manifestations of the same problem, namely focal cerebral vasospasm.

It is generally felt that microinfarctions and/or microhaemorrhages in the cerebral cortex are the most likely triggers of seizures. Their cause is debated but it seems likely that they are secondary to vasospasm produced by endothelial cell injury.

The endothelial cell injury may be caused directly by the blood borne products of an ischaemic placenta (i.e. in exactly the same manner as other end organ ischaemia in preeclampsia). Another theory is that the endothelial cell injury and vasospasm are a form of hypertensive encephalopathy (Redman, 1989). The cerebral circulation auto regulates to maintain a constant blood flow throughout a wide range of mean arterial pressures. The upper and lower limits of the auto regulation are determined by pre-morbid arterial blood pressure. If the auto regulation limits are breached by extreme hypertension then hypertensive encephalopathy ensues with sudden increased cerebral blood flow, arteriolar constriction and dilatation and damage in capillaries and pre-capillaries thus giving ring haemorrhages and localised oedema (Donaldson, 1989).

A third, but no longer so popular theory, is that the seizures of eclampsia are caused by extreme hypertension. This has been undermined by the well documented cases of seizures which occur at relatively low blood pressure and the recognition many of the pathological consequences of eclampsia are rapidly reversible. However, extreme hypertension may well be responsible for the grosser forms of pathological insult to the brain which occur in both pre-eclampsia and eclampsia, and therefore control of hypertension remains vitally important in the management.

\subsubsection{Relationship between pre-eclampsia and eclampsia.}

In many papers there are references to eclampsia as the 'end point', or 'final complication' of the syndrome of pre-eclampsia (Redman, 1989; Peterkin et al. 1992). This is because eclampsia is still commonly perceived as the end of a linear clinical spectrum that stretches from normal pregnancy, through mild hypertension, proteinuric pre-eclampsia and finally eclampsia (see Figure 1.1). This perception arose and is perpetuated by the nomenclature of pre-eclampsia and eclampsia. There is no pathophysiological reason to expect such a linear spectrum and acceptance of this model means that convulsions are not expected unless the woman has severe hypertension, proteinuria and symptoms. 
Figure 1.1. A conventional model of the relationship between pre-eclampsia and eclampsia.

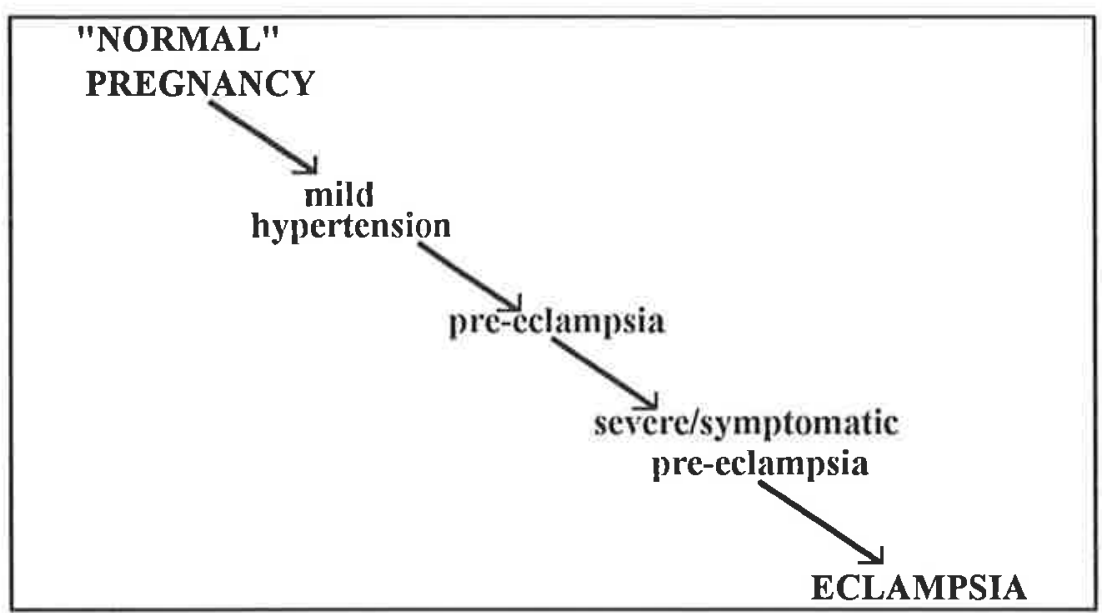

Attempts no explanation at pathological processes but is perpetuated by the nomenclature.

An alternative view consistent with the theories of pathogenesis outlined above is that seizures are one of a range of signs and symptoms caused by widespread endothelial cell damage secondary to an ischaemic placenta. The initial signs and symptoms of the syndrome are therefore dictated by the site and extent of endothelial cell damage and not by a predetermined hierarchy (see Figure 1.2). It is then logical that seizures may precede hypertension or proteinuria. Indeed clinical series have reported up to one in six cases of eclampsia occurring with-out pre-existing proteinuria and hypertension (Campbell \& Templeton, 1980; Nelson, 1955b; Sibai et al. 1986a). The term "pre-eclampsia" is therefore misleading because eclampsia can precede pre-eclampsia.

\subsubsection{Complications of pre-eclampsia and eclampsia.}

If the model of pathogenesis of pre-eclampsia and eclampsia shown in Figure 1.2. is accepted it is logical that damage can occur in any maternal end organs and it becomes possible to understand the diverse nature of the complications of the syndrome. The following (Table 1.4.) is a list of the acute crises and severe complications that can occur in pre-eclampsia and eclampsia adapted from a recent article on the management of preeclampsia (Redman \& Roberts, 1993). 
FIGURE 1.2. The current explanation of the pathogenesis of the maternal syndrome of pre-eclampsia and eclampsia.

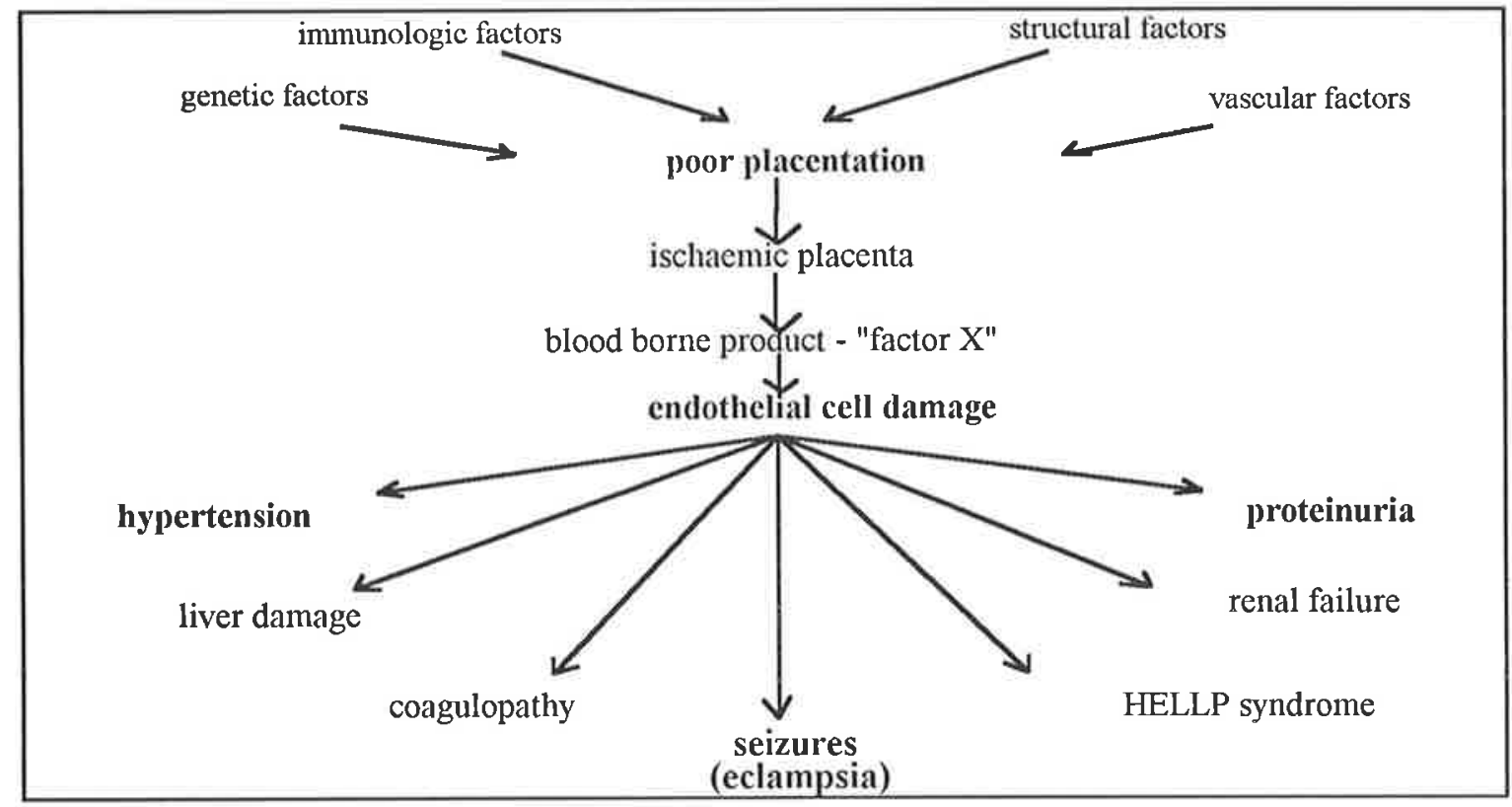

Although still incomplete this model represents more accurately what is now known of the syndrome.

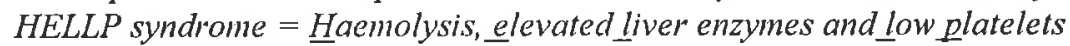

TABLE 1.4. Severe complications and acute crises in pre-eclampsia and eclampsia.

I Neurological

Cerebral haemorrhage

Cortical blindness

Retinal detachment

II Haematological

HELLP syndrome (haemolysis, elevated liver enzyme activity, low platelets)

Disseminated intravascular coagulation

\section{Respiratory}

Pulmonary oedema

Laryngeal oedema

\section{Renal}

Acute renal cortical necrosis

Acute renal tubular necrosis

\section{Fetal}

Abruptio placentae

Intrauterine fetal asphyxia and death 


\subsection{SUB-TYPES OF ECLAMPSIA.}

Eclampsia has a high maternal and perinatal case fatality rate overall but it has long been recognised that not all women with eclampsia are equally affected (Eden, 1922; Nelson, 1955a). This has led to recent interest in trying to identify sub-types of eclampsia that are more or less severe and documenting their clinical characteristics. The two divisions which have been studied are the timing of seizures in relation to the onset of labour and whether the seizures occur at term or preterm.

\subsubsection{Timing of seizures in relation to the onset of labour.}

Eclampsia can be classified as antepartum, intrapartum or postpartum depending of the timing of the onset of seizures in relation to labour. The proportion of all eclampsia in each of these subtypes varies between different case-series (Gedekoh et al. 1981; Pritchard \& Pritchard, 1975). However in the few population based studies the proportions are remarkably similar and are approximately 30\% antepartum, 36\% intrapartum and 34\% postpartum (Corkill, 1961; Wightman et al. 1978; Templeton \& Campbell, 1979).

There is some evidence that women with antepartum seizures have worse maternal and perinatal outcomes than the other types of seizures. In antepartum eclampsia the fetus is subjected to a period of relative hypoxia and this would seem a logical reason for worse perinatal outcome. Women with antepartum eclampsia appear to have higher mortality and morbidity rates as well but the reasons for this are less clear (Odum \& Akinkugbe, 1991; Lopez Llera, 1992). Llopez Llera (1992) has made the most complete study of these different subtypes of eclampsia but he failed to find significant differences between the maternal ages, parity, levels of blood pressure or proteinuria or incidence of coincident chronic illnesses.

There has been controversy about post-partum eclampsia because it is unclear how the syndrome can persist or even get worse after the placenta has been removed. Some authors believe it may have a different cause which involves a transitory insult to the mother; another possible explanation for the improved outcome in postpartum eclampsia is that treatment is more aggressive because there is no need to balance the needs of the mother with the fetus. However neither of these hypotheses has been tested.

Most authors now accept the concept of postpartum eclampsia but may be cautious about making the diagnosis in seizures that occur later in the post-partum period. Late postpartum eclampsia has been variously defined as seizures occurring more than 24 hours (Porapakkham, 1979), 48 hours (Villar \& Sibai, 1988) or 72 hours (Sibai et al. 1980) after 
delivery. Some authors have imposed arbitrary time limits after which they believe it is inappropriate to attribute any seizure to eclampsia (Sheehan \& Lynch. 1973; Lopez Llera, 1967; Jeffcoate \& Scott, 1959) while others have included cases which occurred up to 23 days after delivery (Samuels, 1960). Certainly it would seem prudent to consider the possible differential diagnoses in any case of post partum eclampsia. (Table 1.5.)

TABLE 1.5. The differential diagnoses of eclampsia.

I Cerebrovascular accidents
Cerebral venous thrombosis
Cerebral arterial occlusion
Cerebral arterial embolism
Intracerebral haemorrhage
II Hypertensive disease
Hypertensive encephalopathy
Pheochromocytoma
II Space occupying central nervous system lesions
Brain tumour
Brain abscess
IV Infections diseascs
Meningitis
Encephalitis
V Metabolic diseases
Hypoglycaemia
Hypokalemia
Water intoxication
VI Ejilepsy

\subsubsection{Gestational age at onset of seizures.}

The proportion of eclampsia that occurs preterm ( $\leq 37$ weeks gestation) is between $53 \%$ and $75 \%$ in different population-based studies (Templeton \& Campbell, 1979; Moller \& Lindmark, 1986; Wightman et al. 1978). Another 5-10\% occur at or before 30 weeks gestation (Campbell et al. 1983; Konje et al. 1992; Porapakkham, 1979).

It is well established and not surprising that perinatal outcome is much worse in the preterm group as prematurity itself is one of the major causes of poor perinatal outcome (Neutra, 1975; Sibai et al. 1983). However, in their population-based study of eclampsia in Sweden Moller and Lindmark (1986) found that the maternal mortality and morbidity were also worse for women who had their eclamptic seizure preterm. There were no significant 
differences in the age, parity or maximum blood pressures between the preterm and term groups and Moller hypothesised that the term group was less severely affected because they had a shorter period of sub clinical disease as evidence by lower proportion of infants with growth retardation. A higher maternal mortality rate in preterm eclampsia has also been reported from Mexico which was statistically significant in the very preterm group ( $\leq 30$ weeks gestation) (Lopez Llera et al. 1976)

It is important to note that these two commonly used divisions (gestation at onset and timing in relation to labour) are not mutually exclusive. Most of the preterm seizures will occur antepartum and, intrapartum and postpartum seizures are more likely to occur at term. Unfortunately no study has been done which has taken into account this overlap and tried to dissect out whether prematurity or occurrence before labour is more important.

\subsection{MANAGEMENT OF ECLAMPSIA AND PRE-ECLAMPSIA.}

Women with pre-eclampsia and eclampsia have over the centuries "been blistered, bled, purged, packed, lavaged, irrigated, punctured, starved, sedated, anaesthetised, paralysed, tranquillised, rendered hypotensive, drowned, been given diuretics, had mammectomy, been dehydrated, forcibly delivered, and neglected" (Zuspan \& Ward, 1964). The sheer number of different remedies attempted indicates a lack of understanding of the condition and how to treat it. The sizeable literature on treatment of pre-eclampsia and eclampsia sadly contains very few large, rigorous, controlled trials and consists instead mainly of expositions of methods used at particular institutions.

Logically, the only definitive treatment of pre-eclampsia and eclampsia is termination of the pregnancy and removal of the cause of the syndrome which lies in the placenta. All other methods of treatment are symptomatic, aiming to prolong pregnancy and minimise maternal and perinatal morbidity and mortality (Collins \& Wallenburg, 1989); they do not alter the underlying progression of the disease process (Roberts \& Redman, 1993).

\subsubsection{Current methods and controversies.}

All management strategies should consist of the key elements of; antenatal screening to pick up early signs of the syndrome, monitoring mother and fetus to assess severity and progress, control of maternal hypertension, prevention and/or treatment of seizures and optimal timing of delivery. The exact methods used vary between individuals and countries (Hutton et al. 1992; Trudinger \& Parik, 1982; Lindberg \& Sandstrom, 1981; Lewis et al. 1980) however both the United States of America and Australia have recently produced consensus 
statements on the management of hypertension in pregnancy (National High Blood Pressure Education Program Working Group, 1990; ASSHIP, 1993).

Routine antenatal screening for pre-eclampsia consists of screening for hypertension, proteinuria and excessive weight gain. Once any of these elements are recognised, further monitoring depends on resources but ideally consists of monitoring for hyperuricaemia, thrombocytopenia, abnormal liver enzyme activity and coagulation status. Fetal monitoring includes assessment of fetal growth and monitoring for signs of fetal hypoxia (Redman \& Roberts, 1993).

Hypertension is the most recognised sign of pre-eclampsia and eclampsia. Effective use of antihypertensives to control blood pressure and therefore prevent cerebral haemorrhage is thought to be one of the major factors in the reduction of maternal mortality from eclampsia and pre-eclampsia (Rubin, 1986). Different antihypertensives are used in different institutions and countries, but methyl dopa, hydralazine and labetalol are amongst the favourites everywhere (Hutton et al. 1992; Trudinger \& Parik, 1982; Lindberg \& Sandstrom, 1981; Lewis et al. 1980). It appears that as long as each drug is used in appropriate dosage there is little scientific basis for choosing one over any of the others (Collins \& Wallenburg, 1989), although angiotensin converting enzyme inhibitors have to be avoided because of their adverse effects on the fetus (Rosa et al. 1989).

The question of how to prevent seizures in a women with established pre-eclampsia has been controversial. Some authors believe that in addition to rigorous treatment of hypertension, all women with severe pre-eclampsia should be given prophylactic anticonvulsants (Robson et al. 1990; National High Blood Pressure Education Program Working Group, 1990). However others have reported similarly good outcomes while restricting the use of anticonvulsants to after the onset of seizures (Chua \& Redman, 1991; Ramsay et al. 1994).

There has also been considerable debate about which drug to use. American obstetricians have for a long time advocated the use of magnesium sulphate (Pritchard et al. 1984; Sibai, 1990b; Dinsdale, 1988) although it has no anticonvulsant properties (Donaldson, 1986; Kaplan et al. 1988). However because the seizures seem to be related to cerebral vasoconstriction the use of magnesium sulphate can be justified by its action as a cerebral vasodilator (Sadeh, 1989). Diazepam, chlormethiazole and, more recently, phenytoin are also commonly used. There have been few controlled trials and those that have been completed have been small and inconclusive (Dommisse, 1990; Crowther, 1990; Appleton et al. 1991). A large multi-centre controlled trial has just been completed in the developing 
world which could provide the first good scientific evidence on which to base drug choice (Duley, 1992b). Efforts are also underway to establish a multi-centre trial comparing magnesium sulphate with nimodipine (a specific cerebral vasodilator).

\subsubsection{Possible preventive measures}

Greater understanding of the pathogenesis of pre-eclampsia and eclampsia should lead to more rational treatment. It is also hoped that there will be new advances leading to interventions that can prevent pre-eclampsia.

Part of the chain of actions initiated by endothelial cell damage is platelet activation and recently great hope has been placed in the possible role of antiplatelet therapy in the prevention or treatment of pre-eclampsia. Although initial trials of low dose aspirin were promising (Imperiale \& Petrulis, 1991; Beaufils et al. 1985; Wallenburg et al. 1983), more recent, larger, multi-centre studies indicate that while aspirin may have a role in certain high risk groups, it is not the panacea that was hoped for (Anonymous, 1993; Sibai et al. 1993; CLASP Collaberative Group, 1994). Currently trials are being conducted on fish oil and calcium supplementation.

\subsection{THE PROGNOSIS OF ECLAMPSIA.}

\subsubsection{Future pregnancies.}

There is conclusive evidence that eclampsia or pre-eclampsia in a first pregnancy is associated with a higher risk of pre-eclampsia in subsequent pregnancies (Eskenazi et al. 1991; Hargood \& Brown, 1991; Lopez Llera \& Hernandez Horta, 1974; Mehta \& Young, 1987; Adelusi \& Ojengbede, 1986; Sibai et al. 1986b; Campbell et al. 1985). The degree of increased risk has varied in different studies. After a first affected pregnancy the incidence of eclampsia is between $0 \%$ and $21 \%$ (Sibai et al. 1992; Adelusi \& Ojengbede, 1986; Chesley, 1978b) and of pre-eclampsia is between $12 \%$ and $51 \%$ (Sibai et al. 1992; Lopez Llera \& Hernandez Horta, 1974; Chesley, 1978b). The magnitude of increased risk does seem to be affected by the gestational age in the first pregnancy at which pre-eclampsia or eclampsia occurred. Early onset (i.e. before 30 completed weeks gestation) preeclampsia/eclampsia is associated with a higher risk of pre-eclampsia in subsequent pregnancies (Sibai et al. 1992; Sibai et al. 1986b; Sibai et al. 1991; Lopez Llera \& Hernandez Horta, 1974).

Women with severe pre-eclampsia or eclampsia in their first pregnancy are also at an increased risk of developing other obstetric complications such as placental abruption and 
premature delivery and they have higher incidences of fetal growth retardation and perinatal mortality in their future pregnancies (Sibai et al. 1986b; Lopez Llera \& Hernandez Horta, 1974; Adelusi \& Ojengbede, 1986).

\subsubsection{Long term maternal health.}

Many of the earlier studies on long term maternal health following eclampsia contained the pitfalls discussed in the section 1.3.1; they used different diagnoses, applied to widely different populations and the duration of follow-up was variable (Chesley, 1978b). These studies reported incidences of chronic hypertension of between 0 and $78 \%$ and led some authors to conclude that pre-eclampsia caused chronic hypertension. More recently several larger, well conducted, long term follow-up studies have been done which provide the basis of the following conclusions (Chesley et al. 1976; Sibai et al. 1992; Bryans, 1966).

There appears to be no increase in subsequent chronic hypertension in women who had eclampsia only in their first pregnancy compared with the general population (Bryans, 1966; Chesley et al. 1976; Carleton et al. 1988). However women who have recurrent preeclampsia do appear to have a high rate of chronic hypertension particularly if they have had early onset disease (Chesley et al. 1976; Sibai et al. 1991). Chesley summarised his findings by saying that "Eclampsia neither is a sign of latent essential hypertension nor causes hypertension. Hypertensive pregnancies following eclampsia indicate the probability of later chronic hypertension but do not cause it" (Chesley et al. 1976).

Chesley's long term follow-up of patients found an unexpectedly high incidence of diabetes in women many years after their eclamptic episode. The prevalence of diabetes was 2.5 times the expected prevalence in primiparous eclamptic women and 4 times the expected rate in multiparous eclamptic women (Hibbard, 1978). Sibai found that 5.6\% of women who were pre-eclamptic in their first pregnancy had diabetes at their 10 year follow-up but this was not significantly different from the control group of women who remained normotensive in their first pregnancy (Sibai et al. 1986b).

\subsection{THE NEED FOR A NATIONAL SURVEY.}

\subsubsection{The purpose.}

Eclampsia is now a rare condition in the United Kingdom, but it remains one of the major causes of maternal mortality both in the United Kingdom and world-wide. Apart from its very significant contribution to maternal mortality relatively little is known about the 
condition. Few population based studies have been done despite repeated calls for their need (Davies, 1971; MacGillivray, 1983a; Redman, 1988).

The current incidence of eclampsia in the United Kingdom is not known nor are the maternal or perinatal case fatality rates. The first and only national review of eclampsia in the United Kingdom was completed by Eden in 1922 (Eden, 1922). Eden estimated an incidence of eclampsia in $4 \%$ of hospital labours and a case fatality of $22 \%$ (Eden, 1922). Since that time there have been several smaller studies which have showed much better outcomes in smaller geographical areas; (Templeton \& Campbell, 1979; Wightman et al. 1978) but in the United Kingdom there are no systematically collected population based data which allow us to assess with confidence either the incidence of eclampsia or the rates of morbidity and mortality associated with it.

Few UK clinicians see enough cases of eclampsia to gain an overview of the spectrum of the problems associated with the condition. This may contribute to the fact that in 1985-87 over three quarters $(81 \%)$ of maternal deaths in the United Kingdom from hypertensive disorders of pregnancy (eclampsia and pre-eclampsia) were judged to reflect substandard care, and most of these (70\%) involved specialist services in hospitals. (DHSS et al. 1991) To make progress in the management of eclampsia we need to know the true scale of the problem and its sequelae and also to aggregate the scattered experiences of specialists around the country to create a corporate body of information available to all.

\subsubsection{The plan.}

With the above in mind it was decided to conduct a population based study of eclampsia in the United Kingdom. There were two major aims: to derive basic current epidemiological data about eclampsia and, to gain a realistic overview of eclampsia and its variants.

The only recent studies of eclampsia with large numbers of cases were either, from the developing world where antenatal care was poorly established (Lopez Llera, 1982; Konje et al. 1992; Agobe et al. 1981; Porapakkham, 1979; Neutra, 1973) or, from a single centre collected over a long time period (Pritchard et al. 1984; Sibai, 1990a). The case-fatality and complication rates in these studies have questionable relevance in the United Kingdom. The only large study in the UK occurred over 70 years ago (Eden, 1922).

A small study in a randomly selected area could establish the incidence and case fatality rates of eclampsia in that area and, by extrapolating, in the whole of the UK. However the study would include only a small number of women which would have considerably 
weakened the power of the statistical analysis particularly when comparing subgroups of eclampsia.

The aim was therefore to collect all cases of eclampsia in the UK and compare them with the general maternity population using national statistics thereby producing a population based study of eclampsia. However, very high response rates and complete data collection would be necessary to ensure the validity of epidemiological indices and the size of a national study involved substantial logistical problems. The advantage of a national study was that it would allow collection of a large number of cases in a single year.

There was no precedent for a national study of an obstetric complication and it was uncertain whether or not it would be possible. A pilot study was therefore done to test the feasibility of a national study and its methodology. A description of the pilot study appears in Chapter 2.

\subsubsection{The prior hypotheses.}

Prior to the start of the study the basic aims and prior hypotheses were established and these are listed in Table 1.3. below.

The introduction of antenatal care and screening for pre-eclampsia has been credited with reducing the incidence of eclampsia (section 1.3.2.3.). The mechanism for this is presumed to be through the early identification of signs of pre-eclampsia and the institution of appropriate intervention to prevent seizures. It was therefore felt that women who developed eclampsia in the United Kingdom (where comprehensive antenatal care is offered) may not avail themselves of the care, or develop eclampsia at an early gestation when it is traditional to have visits at monthly rather than more frequent intervals. This led to the formulation of the first hypothesis.

Although the traditional understanding is that most eclamptic seizures are preceded by preeclampsia the current theory of pathogenesis implies that some women will develop seizures before any other signs of the pre-eclamptic syndrome (see section 1.4.3). This and the observations of unheralded eclampsia in up to 1 in 6 women in hospital based case series led directly to the second hypothesis listed below. 
Previous population based studies reported variable proportions of women who have preterm seizures (section 1.5.2). The third hypothesis predicted that a large proportion were preterm.

In section 1.3.6.1 the increased incidence of eclampsia in older and multiparous women was discussed. The fourth and fifth hypotheses in Table 1.3. were formulated because it was felt that both these groups were likely to be more susceptible to developing the complications associated with eclampsia.

The final hypothesis was formulated from the impression of clinicians at the John Radcliffe hospital. They felt that women who developed postpartum eclampsia without having established proteinuria and hypertension prior to delivery were relatively unaffected by their illness.

TABLE 1.6. Aims and prior hypotheses of a national descriptive study of eclampsia.

\section{Aims}

1. To describe all cases of eclampsia that occur in the United Kingdom during one year.

2. To measure the incidence of eclampsia and the associated maternal and perinatal case fatality rates in the United Kingdom; and to document the maternal and perinatal morbidity associated with eclampsia.

3. To document the current methods of management and treatment of eclampsia and to determine what proportion of cases occur despite treatment.

\section{Prior hypotheses}

1. A significant proportion of eclampsia occurs in woman who have had a long interval between formal screening checks for the signs of pre-eclampsia.

2. Eclampsia occurs in some women without prodromal symptoms and signs.

3. A significant proportion of eclampsia occurs in women at or before 32 weeks gestation.

4. There is greater morbidity from eclampsia amongst older women.

5. There is greater morbidity from eclampsia amongst multiparous women.

6. Post-partum eclampsia which occurs without pre-existing signs of severe pre-eclampsia is a relatively benign condition. 
CHAPTER 2.

\section{THE PILOT STUDY}




\subsection{INTRODUCTION.}

At the end of 1990 planning began for the national study of eclampsia. The need for such a study has been discussed in Chapter 1. A pilot was conducted in two Regional Health Authorities of the National Health Service in 1991 to develop the most appropriate methods for the national study.

The aims of the pilot study were to test the methodology for the national study and to collect descriptive data on eclampsia in the two regions. The pilot allowed assessment of the likely size of a national study; tested the feasibility of the proposed case-notification system and ascertained the quality of data obtainable through hospital case-note review and a general practitioner questionnaire. It also enabled the amount and type of information collected to be refined and gave the investigator experience and confidence in analysing and interpreting descriptive epidemiological data prior to the start of the main study. The descriptive data were of secondary importance because a more powerful data set was expected from the main study.

\subsection{METHODS.}

The Oxford and North Western Regional Health Authorities of the National Health Service were chosen for the pilot study. They were geographically accessible and consultants in the two regions had expressed interest in the prospect of a national study.

The Oxford region extends from Kettering in Northamptonshire to Windsor and Reading in Berkshire and includes 11 obstetric units. There are approximately 35,500 maternities per year in the area (OPCS, 1993). The North-Western Regional Health Authority is based around greater Manchester but extends as far as Lancaster in the north. There are 19 consultant obstetric units in the Authority and approximately 59,000 deliveries per year (OPCS, 1993).

\subsubsection{Ethics committee approval.}

Plans for the pilot study were discussed at the Regional Subcommittees in Obstetrics and Gynaecology in the North-Western and Oxford Regional Health Authorities. Consultant representatives of each hospital with maternity beds in the two regions were present and agreed to participate. The study involved retrospective data collection only and no contact was made with the women nor did the conduct of the survey affect their management in any way at all. Many consultants felt that ethics committee approval was not required because of the above factors. When consultants expressed concern, or where individual hospital 
policy required it, then local and/or hospital ethics committee approval was sought. In all hospitals and districts where formal ethics committee approval was sought it was received.

\subsubsection{Collection of notifications.}

The first task was to develop a method for notifications of cases of eclampsia that was systematic, comprehensive and applicable on a national scale. There was no comprehensive system of routinely collected maternity data in the United Kingdom from which names and hospital numbers of women with eclampsia could be extracted. The Korner data system had been introduced in the mid-eighties to provide a minimal data set on all maternities but there were major difficulties in establishing the data set on a national scale. In 1991 and 1992 the Korner system was unable to provide useful information for use in the study.

The method chosen to collect notifications was adapted from that used by the British Paediatric Surveillance Unit (BPSU). The BPSU is a national unit which enables paediatricians to participate in the national surveillance of infection related conditions and to promote the study of uncommon childhood disorders. The BPSU received a response rate of approximately $89 \%$ from consultant paediatricians to monthly requests for notifications of specified conditions (BPSU, 1989). The success of the scheme is attributed to the fact that it is used for rare, severe conditions that "stick" in paediatricians memory and participation requires a minimum of effort.

Eclampsia is rare and usually a severe condition so that when it does occur in an obstetric hospital it makes an impact on the entire unit. Consultants and midwives are likely to remember any case of eclampsia for at least 6 months and they were therefore chosen as the primary source for notifications. All requests for information were personalised, clear and required a minimum response in an attempt to maximise the co-operation from busy clinicians.

\subsubsection{Identification of obstetricians and midwives involved.}

An up-to-date list of consultant obstetricians working at each hospital was formed from the "Directory of Emergency and Special Care Units" (Anonymous, 1991) and compared with lists available from the Royal College of Obstetricians and Gynaecologists. This was than checked by a member of the Regional Subcommittee in Obstetrics and Gynaecology and any omissions corrected. The final list included 106 consultants active in obstetrics.

One midwife willing to co-operate with the study was identified from each of the 30 obstetric units in the two regions. This was achieved by writing to the Directors of 
Midwifery Services at each hospital and asking them to nominate a midwife who was willing to act as the midwife liaison. The aims and methodology of the study were outlined and the individual midwives were asked to confirm his/her willingness to participate. There were delays in identifying midwife participants and a complete list was finally obtained in late April 1991.

\subsubsection{Criteria for notification of cases.}

The notification criteria were broad to avoid missing relevant cases, and to understand the range and frequency of causes of seizures in pregnancy so that the most suitable criteria for notifications were used in the main study.

During 1991 participating consultants and midwives were asked to notify any cases of women who had seizures in pregnancy or in the first 10 days post-postpartum irrespective of the cause of seizure. They were further encouraged to notify cases where women had severe pre-eclampsia and a "funny turn" or "query seizure".

The cut off point of 10 days post-partum was chosen because there are very few reported cases of eclamptic seizures occurring later than this (Watson et al. 1983). In addition all women are under the care of a community midwife for the first ten days post-partum. The midwife would be aware of any hospital admission occurring in that time even if the woman was cared for entirely by general medical staff. Seizures or admissions occurring later than 10 days post-partum would not necessarily be notified to the caring obstetric team.

\subsubsection{Notification methods.}

The regular requests for notifications consisted of a letter, a reply-paid envelope and a notification form. The letter explained the aim of the study and outlined the notification criteria. Participants were asked to fill out and return the notification form regardless of whether or not they had seen a woman with eclampsia. If the respondents had not seen any women with eclampsia then they simply marked the "No" box and returned the form. If they did have a case to report then they were to supply the woman's name, hospital number and her general practitioner's name and address. They were further asked to nominate someone who would be willing to help obtain access to the case-notes so that a case-note review could be completed. Midwives sending in notifications were asked to supply the name of the consultant responsible for the patient.

Mail-outs were sent to consultants in April, July, September and December and to midwives in July, September and December of 1991. A record was kept of all replies. A reminder 
letter (including a further notification form and reply paid envelope) was sent to all consultants and midwives who had not responded in the six weeks following the July and September mail-out. The reminder mail-out was instituted after a poor response from consultants to the April mailing. To avoid confusion with the national study which was then underway, no reminder was sent following the December mail-out.

This regular system was complemented by making notification forms freely available in the participating hospitals so that "on the spot" notifications could also be sent. Before the start of the study, posters were distributed to each of the participating hospitals for display. The posters listed notification criteria and urged junior hospital doctors and midwives to notify any cases of eclampsia and had notification forms attached with a free-post address and telephone number.

\subsubsection{Collection of clinical data.}

Once a notification was received arrangements were made to travel to the hospital concerned so that a detailed review of the case-notes could be completed. The index casenotes were records from the hospital in which the woman had the first eclamptic seizure or, if her seizure was in the community, then the hospital at which she was first seen after her eclamptic seizure. Any case-notes from other hospitals pertaining to the eclamptic episode are referred to as secondary case-notes.

\subsubsection{Criteria for inclusion in eclampsia series.}

It was necessary to strike a balance between including all legitimate cases of eclampsia and having the series diluted by information from women with seizures due to other causes.

For the purposes of the pilot eclampsia was defined as the occurrence of seizures during pregnancy or in the first 10 days post-postpartum when the patient had at least two of the following characteristics within 24 hours before or after the seizures: hypertension, proteinuria, thrombocytopenia and/or elevated plasma aspartate transaminase level.

Hypertension was defined by a booking diastolic pressure of $<90 \mathrm{~mm} \mathrm{Hg}$, a maximum diastolic of $\geq 90 \mathrm{~mm} \mathrm{Hg}$ and a diastolic increment of $\geq 25 \mathrm{~mm} \mathrm{Hg}$ (Redman \& Jefferies, 1988).

Proteinuria was defined as $\geq++$ protein in a random sample or $\geq 0.5$ grams in a 24 hour collection.

Thrombocytopenia was defined as a platelet count of less than $100 \times 10^{9} / 1$.

An elevated plasma aspartate transaminase (AST) was defined as a level $\geq 42 \mathrm{IU} / \mathrm{l}$. 
It was initially assumed that these criteria would provide an unambiguous system for identifying cases. However it became obvious that it would exclude cases that were felt to be eclampsia. One of the major aims was to derive the incidence of eclampsia so it was clearly undesirable to exclude valid cases. The parameters were altered and cases were included in the pilot study as "probable eclampsia" if; the women had one of hypertension, thrombocytopenia or elevated AST or proteinuria, there was no other explanation for the seizure and the treating obstetric team had treated them for eclampsia; or if they met all the criteria but there was another factor that complicated the diagnosis (such as a remote history of epilepsy). This category of "probable eclampsia" was refined further before its use in the main study. (see Chapter 3 )

\subsubsection{Hospital case-note data.}

In cases of seizures with a cause other than eclampsia a note was made of the diagnosis but detailed information was not collected.

In cases of eclampsia information was collected onto a pre-prepared questionnaire covering eight major categories. The categories were: completeness of information, social and demographic factors, antenatal care, prodromal signs and symptoms, features of eclampsia, features of delivery, maternal outcome and fetal outcome.

Detailed information on blood pressures, presence of proteinuria and biochemical parameters was recorded. Information was gathered from the doctors' notes, midwifery notes, observation charts, delivery and anaesthetic records and intensive care notes to ensure maximal data retrieval.

\subsubsection{General practitioner questionnaire.}

Information was obtained from the general practitioners of affected women in order to obtain a complete record of the antenatal care (general practitioners often supervise the majority of antenatal care in the United Kingdom) and because they are often in a better position to comment on residual minor morbidity than their hospital based colleagues. The questionnaire was sent to the general practitioners after hospital case-notes had been received. A reply paid envelope was enclosed with all questionnaires and a record was kept of which general practitioners had responded. 


\subsubsection{Proposed patient questionnaire.}

It was originally planned to send a questionnaire to all affected women after their eclamptic seizure. The aim was to collect information on women's fears and expectations following eclampsia particularly about how they viewed future pregnancies.

There was resistance to this idea from some consultants and general practitioners. Many questioned the ethics of asking the woman for information without the treating doctors prior permission but methods were devised to overcome this. Others doctors were resistant to the idea because they believed that sending the women a questionnaire would cause unnecessary distress by reactivating memories of a difficult and stressful period (particularly if the woman's infant had died). A few expressed concern that a patient questionnaire may stimulate the women to question their management and increase the likelihood of medical litigation against the hospital or doctors. Owing in part to the continued reluctance but also because of the demands of establishing the national study, the patient questionnaire was not carried out. In retrospect it is felt that a valuable opportunity was lost.

\subsubsection{Entry of data onto computerised data base.}

At the completion of the pilot the case-note data and the general practitioner questionnaires were collated. The data were transformed into numerical and logical data and recorded on a data sheet. The data were entered into a computer database using the software programme DBASE IV.

\subsubsection{Analysis of clinical data.}

The data were transferred from DBASE IV to EPI INFO 5 and were analysed using the statistical package contained within EPI INFO (Dean et al. 1990).

On producing basic frequency tables it became obvious that there were a significant number of errors in the database. These arose from transcription or keying errors. In all cases suspicious data were checked and corrected by referring to the original questionnaire and data sheets. In a few instances there were errors that appeared on the questionnaire sheet due to inaccurate retrieval from the case-notes. There was no longer access to the original case-notes so in instances of obvious error the wrong value was removed and replaced with a missing datum value. Alterations were made to the main study protocol to minimise these problems. 
Denominator data on the number of maternities and live and still births in the two Regional Health Authorities were extracted from the OPCS publication Birth Statistics 1991 (OPCS, 1993).

Basic frequency tables were produced for categorical data and means and standard deviations (SD) for continuous data. Statistical analysis employed the Chi squared test with Yates correction ( 1 degree of freedom unless otherwise stated) and Fishers exact test. Where relevant, relative risks were calculated and the $95 \%$ confidence interval for relative risks are shown in brackets.

\subsection{RESULTS.}

\subsubsection{Results of methodological aspects.}

\subsubsection{Response to requests for notifications.}

The regular requests for notifications received a $45 \%$ response rate from consultants and a $72 \%$ response rate from midwives. The details of the responses to individual mail-outs can be seen in Table 2.1. The mail-outs to consultants and midwives in which a reminder letter was sent to non responders after six weeks (July and September) obtained a much better return rate compared with those with no reminder.

TABLE 2.1. Pilot study: Responses of consultants and midwives to mail-outs.

\begin{tabular}{lcccc}
\hline & \multicolumn{2}{c}{ Consultants } & \multicolumn{2}{c}{ Midwives } \\
& No. & $\%$ & No. & $\%$ \\
\hline April & 29 & 27 & $*$ & $*$ \\
July & 68 & 63 & 28 & 93 \\
September & 69 & 64 & 21 & 70 \\
December & 27 & 25 & 16 & 53 \\
\hline Total & $193 / 432$ & 45 & $65 / 90$ & 72 \\
\hline \multicolumn{4}{r}{}
\end{tabular}

The number of responses from individual hospitals was derived by combining responses from consultants and midwives. One hundred per cent of hospitals with maternity beds in the North-Western and the Oxford regions replied at least once during 1991. Eighty-seven percent of hospitals replied at least three times during the year and at least one reply to each mail-out (i.e. 4 replies during the year) were obtained from $47 \%$ of the hospitals. 


\subsubsection{Outcome of notifications.}

One hundred and twenty notifications of seizures in pregnancy were received during 1991 as shown in Figure 2.1. One hundred and nineteen (99\%) case-notes were reviewed. There was one set of index case-notes that could not be traced despite repeated visits to the hospital and the case was excluded.

Seventy eight of the reviewed cases were not eligible for inclusion in the analysis. The reasons for exclusion were epilepsy (26), women with severe pre-eclampsia but no seizure (31), women with seizures due to another cause such as space occupying lesions, hypoglycaemia or Wernicke's encephalopathy (13) and women with simple syncope or a "funny turn" but no signs of pre-eclampsia nor a generalised convulsion (8).

Forty-one cases were included in the study and of these $37(90 \%)$ fulfilled the criteria for classical eclampsia and $4(10 \%)$ were included as probable eclampsia. All cases of classical and probable eclampsia were analysed together.

FIGURE 2.1. Pilot study: Outcome of notifications.

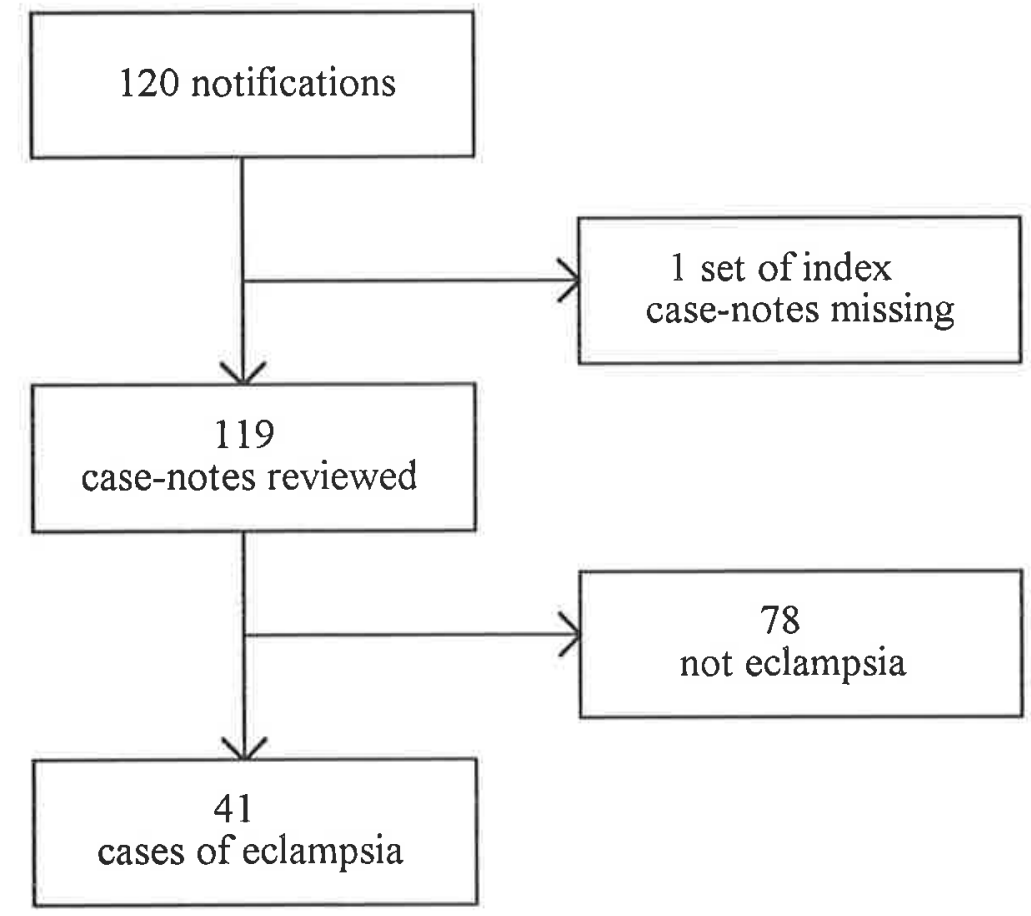

\subsubsection{Completeness of information retrieval.}

Forty-one index case-notes were reviewed. In all cases the doctors and midwives' notes were reviewed as well as observation charts and biochemical, haematological and pathology 
results. In 11 cases women were admitted to intensive care unit and the notes were reviewed but the units' observation charts were not found

In four cases $(10 \%)$ women were transferred to another hospital for further management following eclamptic seizure. The secondary case-notes were not reviewed and the general practitioner questionnaire was returned in only 1 of these 4 cases. The data on complications and morbidity in these women may be incomplete.

The woman's antenatal care co-operation card was reviewed in $26(64 \%)$ of the cases. Twenty-six (64\%) of the general practitioner questionnaires were completed and returned.

\subsubsection{Quality of information retrieved.}

The percentage of missing values in each variable varied considerably. Basic social and demographic data was poorly recorded with mean of $9 \%$ missing values (range $0-22 \%$ ). Details of the eclamptic episode and of delivery were very well recorded (particularly in the midwifery notes); most variables had no missing values and all variables in those categories had less than $6 \%$ missing values (mean $0.5 \%$ missing, range $0-5.7 \%$ ). Details on maternal outcome were well recorded in the hospital case-notes which reflects short-term outcome but in $15(36 \%)$ of cases the general practitioner questionnaire was not completed and thus the information on outcome after discharge was considerably weaker.

The area that was most poorly recorded overall was fetal outcome. Only the mother's casenotes were reviewed and they contained a minimal amount of information concerning the neonate. There were 44 fetuses and in all cases it was possible to ascertain whether the babies were still or live born and their birth weight's. In $8(20 \%)$ Apgar scores were missing.

In all but 1 case $(98 \%)$ it was possible to establish if the baby had been admitted to the special care baby unit. For $16(53 \%)$ of the 30 babies admitted to a unit there was no information about how long they stayed or if they survived to discharge. In $22(50 \%)$ of cases no information was available from either the hospital case-notes or the general practitioner questionnaire on perinatal morbidity. In the main study efforts were made to improve the information on neonatal outcome - this is discussed in section 3.5.5. Given the high percentage of missing information on neonatal outcomes the neonatal case fatality rate derived from this pilot study must be treated with caution. The true value would not be lower than that reported but may well be higher. 


\subsubsection{Results of clinical case-note reviews.}

\subsubsection{Incidence.}

There were 41 cases of eclampsia and 93,819 maternities in the two regions during the calendar year of 1991 giving an incidence of eclampsia of 4.4 per 10,000 maternities. Table 2.2 shows the number of cases and incidence of eclampsia in each region of the pilot study.

TABLE 2.2. Pilot study: The incidence of eclampsia in the Oxford and the North-Western RHA's.

\begin{tabular}{lccc}
\hline & $\begin{array}{c}\text { North Western } \\
\text { Region }\end{array}$ & $\begin{array}{c}\text { Oxford } \\
\text { Region }\end{array}$ & Total \\
\hline No. cases & 24 & 17 & 41 \\
No. maternities* & 57,997 & 35,822 & 93,819 \\
Incidence $/ 10,000$ maternities & 4.1 & 4.7 & 4.4 \\
$(95 \%$ confidence interval) & $(2.5-5.8)$ & $(2.5-7.0)$ & $(3.0-5.7)$ \\
\hline
\end{tabular}

* Data from Birth Statistics 1991 (OPCS, 1993)

\subsubsection{Risk factors}

There were $30(73 \%)$ primiparous and $11(27 \%)$ multiparous women with eclampsia. Thirty-five $(85 \%)$ of the women were aged between 20 and 35 years. Five $(12 \%)$ were teenagers and only one woman was greater than 35 years old ( 42 years).

Of the 11 parous women $7(64 \%)$ had significant risk factors for pre-eclampsia. Three women were pregnant with twins, 2 had a history of pre-eclampsia, 1 had a history of intrauterine growth retardation and 1 was an elderly grand multipara (42 year old woman known to be hypertensive and refusing antenatal care). Four of the 11 multiparous women had no known risk factors for pre-eclampsia.

\subsubsection{Antenatal care and prodromes to eclampsia.}

Three $(7 \%)$ women had concealed pregnancies and received no antenatal care before presentation with eclampsia or delivery. There were $24(59 \%)$ other women whose entire antenatal care records were reviewed (i.e. midwifery, general practitioner and consultant visits) and a summary of these cases is shown in Table 2.3. The traditional pattern of antenatal care in the United Kingdom consists of a booking visit prior to 20 weeks gestation, monthly visits between booking and 30 weeks, fortnightly visits from 30-36 weeks and weekly visits thereafter. Eleven (41\%) women had antenatal care which did not conform to the traditional pattern. Three had no care, 3 booked after 20 weeks gestation and 5 women had less frequent visits than is traditional. 
TABLE 2.3. Pilot study: Features of antenatal care of women with eclampsia.

(Includes all 24 women whose complete antenatal care record was reviewed)

\begin{tabular}{|c|c|c|c|c|c|c|c|c|c|c|}
\hline \multirow{2}{*}{$\begin{array}{l}\text { Study } \\
\text { No. }\end{array}$} & \multirow[b]{2}{*}{ Parity } & \multicolumn{3}{|c|}{ Booking visit } & \multicolumn{3}{|c|}{ Last antenataI visit } & \multirow{2}{*}{$\begin{array}{c}\text { Delivery } \\
\text { Gest }^{n} \\
\text { (wks) }\end{array}$} & \multirow{2}{*}{\begin{tabular}{|c|} 
No. \\
antenatal \\
visits
\end{tabular}} & \multirow[b]{2}{*}{ Outcome } \\
\hline & & $\begin{array}{l}\text { Gest }^{n} \\
(\text { wks })\end{array}$ & $\mathrm{BP}$ & Urine & $\begin{array}{l}\text { Gest }^{n} \\
\text { (wks) }\end{array}$ & $\mathrm{BP}$ & Urine & & & \\
\hline 1 & $P_{0+0}$ & $11+$ & $90 / 70$ & NS & $40+$ & $130 / 70$ & Trace & $41+$ & 15 & $\begin{array}{l}3 \text { days later admitted for induction. Hypertension in labour. IP } \\
\text { seizure. }\end{array}$ \\
\hline 2 & $\mathrm{P}_{n+1}$ & $9 H$ & $120 / 70$ & NS & 36 & $170 / 100$ & +++ & 36 & 9 & Admitted fram clinic with PET \& IUGR. AP seizure. \\
\hline 6 & $P_{n+1}$ & 9 & $110 / 70$ & NS & 36 & $110 / 70$ & NAD & 36 & 17 & $\begin{array}{l}7 \text { days later admitted with ? labour. HT \& proteinuric. AP seizure. } \\
\text { "Probable" eclampsia. }\end{array}$ \\
\hline 8 & $\mathrm{P}_{1+0}$ & 14 & $110 / 70$ & NS & 32 & $110 / 70$ & NS & $33+$ & 9 & 13 days later AP seizure at home. \\
\hline 12 & $\mathrm{P}_{1+0}$ & $7+$ & $108 / 68$ & NS & 36 & $110 / 70$ & NAD & 34 & 10 & $\begin{array}{l}3 \text { days later admitted with placental abruption \& IUD. PP developed } \\
\text { PET and PP seizure }\end{array}$ \\
\hline 15 & $\mathrm{P}_{0+1}$ & 28 & $120 / 70$ & $\mathrm{NAD}$ & 37 & $150 / 90$ & NAD & 37 & 7 & l day later admitted $\&$ induced. IP seizure. \\
\hline 16 & $P_{n+0}$ & 10 & $105 / 55$ & NS & 36 & $100 / 60$ & NAD & 37 & 16 & $\begin{array}{l}8 \text { days later self presented with headache, epigastric pain. } \\
\text { Diagnosed PET AP seizure. }\end{array}$ \\
\hline 18 & $P_{n+n}$ & 7 & $100 / 65$ & NS & 40 & $130 / 80$ & ++++ & $40+$ & 16 & $\begin{array}{l}5 \text { days later admitted in labour. Hypertension, cephalopelvic } \\
\text { disproportion and IP seizure. }\end{array}$ \\
\hline 19 & $P_{0+0}$ & 10 & $110 / 70$ & NAD & 32 & $130 / 80$ & $\mathrm{NAD}$ & $34+$ & 7 & 16 days later AP seizure at home. PET. \\
\hline 22 & $\mathrm{P}_{0+0}$ & 8 & $120 / 7.0$ & NAD & 41 & $140 / 90$ & NS & $41+$ & 17 & 5 days later admitted in labour. PET \& PP seizure. \\
\hline 29 & $\mathrm{P}_{1+0}$ & 12 & $120 / 7.0$ & NAD & $37+$ & $110 / 70$ & NAD & $38+$ & 12 & 6 days later admitted \& induced - twin pregnancy. PP seizure. \\
\hline 34 & $\mathrm{P}_{0+0}$ & 6 & $90 / 60$ & NAD & 36 & $120 / 75$ & $\mathrm{NAD}$ & 36 & 10 & That evening AP seizure at home. On admission symptomatic PET. \\
\hline 39 & $P_{5+1}$ & $15+$ & $184 / 9.2$ & NS & 40 & $240 / 140$ & NS & $40+$ & 11 & $\begin{array}{l}1 \text { day later admitted in labour. IP seizure. Refused medical treatment } \\
\& \text { antenatal care. }\end{array}$ \\
\hline 40 & $\mathrm{P}_{4+4}$ & 12 & $90 / 50$ & NS & 40 & $110 / 70$ & NAD & 40 & 9 & $\begin{array}{l}4 \text { days later admitted in labour. PP PET \& seizure (history of PET in } \\
\text { previous pregnancy) }\end{array}$ \\
\hline
\end{tabular}

$\mathrm{BP}$ - blood pressure, Gest ${ }^{\mathrm{n}}$ - gestational age, AN - antenatal, NS - no specimen, NAD - no abnormality detected, AP - antepartum, IP - intrapartum,

PP - post partum, PET - pre-eclampsia, IUD - intrauterine death, IUGR - intnauterine growth retardation, APH - antepartum haemorrhage, GP - family practitioner.. 
Table 2.I. cont. Pilot study: Features of antenatal care of women with eclampsia.

(Includes all 24 women whose complete antenatal care record was reviewed)

\begin{tabular}{|c|c|c|c|c|c|c|c|c|c|c|}
\hline \multirow{2}{*}{$\begin{array}{l}\text { Study } \\
\text { No. }\end{array}$} & \multirow{2}{*}{$\begin{array}{l}\text { Gravid } \\
\text { Status }\end{array}$} & \multicolumn{3}{|c|}{ Booking visit } & \multicolumn{3}{|c|}{ Last antenatal visit } & \multirow{2}{*}{$\begin{array}{c}\text { Delivery } \\
\text { Gest }^{n} \\
(w k s)\end{array}$} & \multirow{2}{*}{\begin{tabular}{|c|} 
No. \\
antenatal \\
visits
\end{tabular}} & \multirow[b]{2}{*}{ Outcome } \\
\hline & & $\begin{array}{l}\text { Gest }^{n} \\
\text { (wks) }\end{array}$ & $\mathrm{BP}$ & Urine & $\begin{array}{l}\text { Gest }^{n} \\
\text { (wks) }\end{array}$ & BP & Urine & & & \\
\hline$\overline{43}$ & $P_{0+0}$ & 33 & $120 / 60$ & NAD & $39+$ & $120 / 70$ & NS & $39+$ & 7 & $\begin{array}{l}\text { That evening admitted ? early laboun. PP PET \& seizure. } 13 \text { yrs old } \\
\text { late booker. }\end{array}$ \\
\hline 44 & $P_{n+1}$ & $6+$ & $140 / 90$ & NAD & $29+$ & $140 / 88$ & NAD & $31+$ & 20 & $\begin{array}{l}14 \text { days later GP to home - headache \& epigastric pain - PET. } \\
\text { Admission - AP seizure. }\end{array}$ \\
\hline 47 & $P_{1+0}$ & $9+$ & $100 / 50$ & NAD & 33 & $110 / 75$ & NAD & $34+$ & 12 & 14 days later admitted ? early labour. Fulminating PET. PP seizure. \\
\hline 50 & $P_{n+0}$ & 15 & $120 / 60$ & NAD & 37 & $130 / 90$ & $+1+$ & 38 & 9 & $\begin{array}{l}\text { Admitted from clinic for PET. } 2 \text { days later normal delivery but PP } \\
\text { seizure. }\end{array}$ \\
\hline 51 & $P_{n+0}$ & $10+$ & $100 / 60$ & NAD & 30 & $120 / 70$ & NAD & 31 & 7 & $\begin{array}{l}7 \text { days later admitted with APH. Developed PET in hospital AP \& } \\
\text { PP seizures. }\end{array}$ \\
\hline 52 & $P_{n+0}$ & 21 & $110 / 70$ & NAD & 37 & $120 / 80$ & Trace & $37+$ & 9 & $\begin{array}{l}\text { Admitted from clinic with IUGR \& oedema. Late PF seizure. } \\
\text { "Probable" eclampsia }\end{array}$ \\
\hline 65 & $P_{n+0}$ & 16 & $110 / 60$ & NAD & $25+$ & $120 / 70$ & NAD & $27+$ & 5 & 14 days later AP seizure at home. On admission severe PET. \\
\hline 68 & $P_{n+0}$ & 6 & $100 / 60$ & NS & 37 & $130 / 90$ & NAD & $37+$ & 13 & $\begin{array}{l}4 \text { days later admitted in spontaneous labour. PP developed PET \& } \\
\text { late PP seizure. }\end{array}$ \\
\hline 73 & $P_{0+0}$ & 6 & $100 / 60$ & NAD & 34 & $110 / 65$ & NAD & $37+$ & 10 & $\begin{array}{l}23 \text { days later presented with abruption \& IUD. Induced, developed } \\
\text { PET \& PP seizure. }\end{array}$ \\
\hline 80 & $P_{1+0}$ & $11+$ & $100 / 60$ & NAD & $32+$ & $150 / 100$ & ++++ & $32+$ & 11 & $\begin{array}{l}\text { Admitted from clinic for PET. PP seizure. (history of PET in } \\
\text { previous pregnancy). }\end{array}$ \\
\hline
\end{tabular}

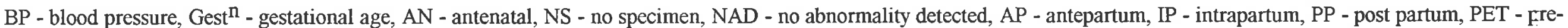
eclampsia, IUD - intrauterine death, IUGR - intrauterine growth retardation, APH - antepartum haemorrhage, GP - general practitioner. 
The mean (standard deviation) of the first blood pressure measurements taken in pregnancy was 113 (12.1) $\mathrm{mm} \mathrm{Hg}$ systolic over 67 (8.3) $\mathrm{mm} \mathrm{Hg}$ diastolic. Seventeen women had their blood pressure taken within the hour before the onset of fits and the mean for this last blood pressure measurement was 169 (20.9) $\mathrm{mm} \mathrm{Hg}$ systolic over 105 (9.3) $\mathrm{mm} \mathrm{Hg}$ diastolic. Nineteen $(46 \%)$ women had ++ or more of proteinuria recorded before the onset of seizures.

Twenty-seven $(66 \%)$ women were symptomatic prior to the first seizure. The most common symptom was headache which was present in 26 . Nine women had epigastric pain and 6 complained of changes in their vision before the onset of seizures. Data are missing in one case.

Thirty-two (78\%) had their first fit while in a consultant obstetric unit, the remainder (9 $22 \%$ ) had their first fit at home. Overall $16(39 \%)$ women in this series did not have antenatal pre-eclampsia recorded before their first seizure.

The mean maximum blood pressure for all women taken at any time during the eclamptic illness was 182 (23.7) $\mathrm{mm} \mathrm{Hg}$ systolic over 119 (11.3) $\mathrm{mm} \mathrm{Hg}$ diastolic. Thirty-three $(80 \%)$ women had ++ or more of proteinuria at some time during their eclamptic illness.

\subsubsection{Type of eclampsia.}

Sixteen (39\%) of the women had antepartum eclampsia, $7(17 \%)$ intrapartum and $18(44 \%)$ had postpartum eclampsia (Table 2.4.). Three (7\%) of the postpartum cases were late onset, the first seizure occurring more than 24 hours after delivery. Twenty-four (58\%) women had multiple seizures.

\subsubsection{Gestational age.}

Twenty-one women (51\%) were preterm (i.e. had a gestational age of less than 37 completed weeks) at the time of their first seizure. 15 of the $16(94 \%)$ women with antepartum eclampsia were included in this group (Table 2.4.).

Thirty-two (88\%) women were admitted to hospital before the onset of eclampsia. Nine $(12 \%)$ had their first seizure at home or in the community and almost all $(8-89 \%)$ of these were antenatal and preterm. 
TABLE 2.4. Pilot study: Number of patients with eclampsia by gestational age and type of eclampsia.

\begin{tabular}{ccccc}
\hline $\begin{array}{c}\text { Gestational age } \\
\text { (wks) }\end{array}$ & All women & Antepartum & Intrapartum & Postpartum \\
\hline$<32$ & 6 & 6 & 0 & 0 \\
$32-36^{6}$ & 15 & 9 & 0 & 6 \\
$\geq 37$ & 20 & 1 & 7 & 12 \\
\hline Total & 41 & 16 & 7 & 18 \\
\hline
\end{tabular}

\subsubsection{Investigations.}

The only investigation done routinely in women before eclampsia was a platelet count (see table 2.5.). The percentage of women investigated was not substantially increased even in women hospitalised for at least 24 hours before the seizure.

After the onset of eclampsia the percentage of women who had biochemical tests increased dramatically. In this series the measurement of serum bilirubin was common after the first seizure but only just over half of the women had their plasma transaminase levels measured which is a much more sensitive measure of hepatocellular involvement in preeclampsia/eclampsia.

TABLE 2.5. Pilot study: Investigations carried out before and after first eclamptic seizures.

\begin{tabular}{lcccc}
\hline \multicolumn{1}{c}{ Investigation } & \multicolumn{2}{c}{$\begin{array}{c}\text { Before first seizure } \\
(\mathrm{n}=32) *\end{array}$} & \multicolumn{2}{c}{$\begin{array}{c}\text { After first scizure } \\
(\mathrm{n}=41)\end{array}$} \\
& No. & $\%$ & No. & $\%$ \\
\hline Serum Urea & 15 & 47 & 38 & 93 \\
Serum Creatinine & 13 & 41 & 36 & 88 \\
Serum Bilirubin & 9 & 28 & 33 & 81 \\
Plasma AST & 8 & 25 & 24 & 59 \\
Platelet Count & 25 & 78 & 40 & 98 \\
APTT/INR & 11 & 34 & 35 & 85 \\
\hline
\end{tabular}

*9 women excluded as first seizure was prior to admission to hospital. AST - Aspartate amino transaminase. APTT - Activated partial thromboplastin time. INR - international normal ratio

\subsubsection{Treatment methods.}

Ten $(31 \%)$ of the 32 women who were in hospital before the advent of eclampsia received some form of prophylactic medication. Nine (28\%) women received antihypertensives (labetalol, hydralazine or nifedipine), six had non specific sedatives (pethidine, promethazine 
or oral diazepam) and two women $(6 \%)$ were given a prophylactic anticonvulsant (in both cases phenytoin).

Table 2.6. show the drugs used in the management of eclamptic women. Eight women $(20 \%)$ did not require any antihypertensive medication. Diazepam was the first medication to be used after eclamptic seizures in $27(66 \%)$ cases and was usually given as an intravenous bolus dose.

TABLE 2.6. Pilot study: Medications used in the management of women with eclampsia.

\begin{tabular}{|c|c|c|}
\hline Medications. & No. & $\%$ \\
\hline \multicolumn{3}{|l|}{ INTRAVENOUS } \\
\hline $\begin{array}{l}\text { Anticonvulsants/Sedatives } \\
\text { Diazepam } \\
\text { Phenytoin } \\
\text { Chlormethiazole }\end{array}$ & $\begin{array}{r}31 \\
19 \\
9\end{array}$ & $\begin{array}{l}76 \\
46 \\
22\end{array}$ \\
\hline $\begin{array}{c}\text { Antihypertensives } \\
\text { Hydralazine } \\
\text { Labetalol }\end{array}$ & $\begin{array}{l}26 \\
10\end{array}$ & $\begin{array}{l}63 \\
24\end{array}$ \\
\hline $\begin{array}{l}\text { Others } \\
\text { Paralysis \& intubation } \\
\text { Mannitol } \\
\text { Dopamine } \\
\text { Diuretics }\end{array}$ & $\begin{array}{l}5 \\
3 \\
2 \\
2\end{array}$ & $\begin{array}{r}12 \\
7 \\
5 \\
5\end{array}$ \\
\hline \multicolumn{3}{|l|}{ ORAL } \\
\hline $\begin{array}{l}\text { Anticonvulsants } \\
\text { Phenytoin } \\
\text { Carbamazepine }\end{array}$ & $\begin{array}{l}8 \\
2\end{array}$ & $\begin{array}{r}20 \\
5\end{array}$ \\
\hline $\begin{array}{l}\text { Antihypertensives } \\
\text { Labetalol } \\
\text { Nifedipine } \\
\alpha \text { Methyl dopa }\end{array}$ & $\begin{array}{l}7 \\
5 \\
2\end{array}$ & $\begin{array}{r}17 \\
12 \\
5\end{array}$ \\
\hline
\end{tabular}

\subsubsection{Maternal outcome.}

Twenty-four women (59\%) were in hospital for more than one week following their eclamptic seizure and three $(7 \%)$ of them were still hospitalised 3 weeks later. Twentythree women $(56 \%)$ had at least one major complication during their hospital admission for eclampsia and $14(34 \%)$ had more than one complication. These complications were diagnoses recorded in hospital case-notes and are shown in Table 2.7. 
In 21 cases $(51 \%)$ women were admitted to an intensive care unit. In some hospitals this is routine for the first 24 hours following an eclamptic seizure but 17 of the 21 women were in intensive care for 2 or more days indicating that it was for more than routine observation.

TABLE 2.7. Pilot study: Major complications of women with eclampsia.

\begin{tabular}{lcc}
\hline \multicolumn{1}{c}{ Complication } & No. & $\%$ \\
\hline Disseminated intravascular coagulopathy & 11 & 27 \\
Required ventilatory support* & 10 & 24 \\
HELLP syndrome & 6 & 15 \\
Pulmonary oedema & 4 & 10 \\
Renal failure & 4 & 10 \\
Sepsis & 3 & 7 \\
Adult respiratory distress syndrome* & 2 & 5 \\
Cerebral oedema* & 2 & 5 \\
Illeus & 2 & 5 \\
Acute psychosis & 1 & 2 \\
Cardiac arrest* & 1 & 2 \\
Death complication of the one fatal case & 1 & 2 \\
\hline
\end{tabular}

Table 2.8. shows the outcome of women divided into those with preterm (i.e. $<37$ completed weeks gestation) versus term ( $\geq 37$ weeks) eclampsia. Women who developed eclampsia pre-term were more likely to stay in hospital for over a week, had more major complications and were more likely to be admitted to the intensive care unit than women who developed eclampsia at term. The number in each group is small and as shown below the only statistically significant difference was in hospital stay.

Women with antepartum eclampsia appeared to fare less well than women whose first seizure occurred after the onset of labour as illustrated in Table 2.9. They were more likely to stay in hospital for over a week, had more major complications and were admitted to intensive care units more frequently than women with intra or postpartum seizures. Again the numbers in each group were small and the only difference which was statistically significant was the proportion who required prolonged hospital stay. Interpretation of this is difficult as all antepartum fits occurred pre-term. Due to the small numbers involved it was not possible to stratify the data and still perform meaningful analyses. 
TABLE 2.8. Pilot study: Maternal outcomes by gestation at onset of eclampsia.

\begin{tabular}{|c|c|c|c|c|c|}
\hline \multirow[t]{2}{*}{ Outcome } & \multicolumn{2}{|c|}{$\begin{array}{c}\text { Preterm }(<37 \text { weeks }) \\
\qquad(\mathrm{n}=21)\end{array}$} & \multicolumn{2}{|c|}{$\begin{array}{c}\text { Term ( }(\geq 37 \text { weelss) } \\
(n=20)\end{array}$} & \multirow{2}{*}{$\begin{array}{c}\text { Statistical testing } \\
\text { Chi Square Yates corrected } \\
\text { Relative risk }(95 \% \mathrm{CI}) \\
\end{array}$} \\
\hline & No. & $\%$ & No. & $\%$ & \\
\hline Hospital stay $>7$ days & 17 & 81 & 7 & 35 & $\begin{array}{c}7.12 \mathrm{P}=0.008 \\
2.31(1.23-4.35)\end{array}$ \\
\hline Major complications & 15 & 71 & 8 & 40 & $\begin{array}{c}2.93 \mathrm{P}=0.09 \\
1.79(90.98-3.26)\end{array}$ \\
\hline Admission to ICU & 13 & 62 & 8 & 40 & $\begin{array}{c}1.19 \mathrm{P}=0.275 \\
1.55(0.82-2.91)\end{array}$ \\
\hline
\end{tabular}

TABLE 2.9. Pilot study: Maternal outcomes by type of eclampsia.

\begin{tabular}{|c|c|c|c|c|c|}
\hline \multirow[t]{2}{*}{ Outcome } & \multicolumn{2}{|c|}{$\begin{array}{c}\text { Antepartum } \\
(\mathrm{n}=16)\end{array}$} & \multicolumn{2}{|c|}{$\begin{array}{l}\text { Intra or Postpartum } \\
\qquad(\mathrm{n}=25)\end{array}$} & \multirow{2}{*}{$\begin{array}{c}\text { Statistical testing } \\
\text { Chi Square Yates corrected } \\
\text { Relative risk }(95 \% \mathrm{CI})\end{array}$} \\
\hline & No. & $\%$ & No. & $\%$ & \\
\hline Hospital stay $>7$ days & 14 & 88 & 10 & 40 & $\begin{array}{c}7.22 \mathrm{P}=0.007 \\
2.19(1.31-3.66)\end{array}$ \\
\hline Major complications & 12 & 75 & 11 & 44 & $\begin{array}{c}2.65 \mathrm{P}=0.10 \\
1.70(1.01-2.88)\end{array}$ \\
\hline Admission to ICU & 11 & 69 & 10 & 40 & $\begin{array}{c}2.18 \mathrm{P}=0.13 \\
1.72(0.96-3.08)\end{array}$ \\
\hline
\end{tabular}

There was 1 maternal death which gives a maternal case fatality rate of $2.4 \%$. The woman was a 23 year old primpara who was normotensive when she booked at 9 weeks gestation. She became hypertensive at 30 weeks gestation and was admitted with proteinuric preeclampsia. Her maximum blood pressure before the onset of seizures was 150/100 and she was observed closely but not given any medications. On the morning of the third day of admission she developed headaches, epigastric pain and visual disturbances and a caesarean section was booked. She had two eclamptic seizures before delivery and was given diazepam and phenytoin prior to an emergency lower segment caesarean section, during which her blood pressure was controlled using intravenous labetalol. She had a live female infant weighing 1490 grams who was transferred to the special care baby unit and survived until discharge. The mother had multiple seizures post delivery, she aspirated gastric contents during one of these and was transferred to the intensive care unit. Over the following two weeks her condition progressively deteriorated with unstable blood pressure, aspiration pneumonia and adult respiratory distress syndrome. On day 13 she had a cardiorespiratory arrest and died.

\subsubsection{Fetal/infant outcome.}

There were 38 singleton pregnancies and three twin pregnancies. All three sets of twins were delivered at term and all 6 infants survived. Fifteen $(39 \%)$ of the singleton infants 
were small for gestational age (i.e. less than 10 th centile for gestational age). Sixty five percent of infants (28/43 missing information on 1) required admission to the special care baby unit. Forty-eight percent (21/44) were delivered preterm (before 37 completed weeks gestation) and $36 \%(16 / 44)$ were delivered following antepartum seizures.

There were 2 deaths amongst the 44 fetuses. Both fatalities were intrauterine deaths giving a stillbirth rate of $4.5 \%$ In both cases the stillbirths occurred after placental abruption in women who had been diagnosed as having intrauterine growth retardation. Neither woman had recorded symptoms or signs of pre-eclampsia before the fetal death but both developed post-partum eclampsia. There were no neonatal deaths recorded. However as mentioned in section 3.1.4 the neonatal follow-up on infants was incomplete: of the 26 infants admitted to special care baby units it was only possible to positively confirm that 10 were discharged alive. The fate of the other 16 is unknown.

Infants born pre-term were more likely to be small for gestational age and a greater proportion of them were admitted to a special care baby unit compared with infants born at term (Table 2.11). Only the difference in admission rates to special care baby units was statistically significant: preterm babies had a relative risk ( $95 \%$ confidence interval) of 2.31 (1.30 - 4.11) compared to babies born at term.

Table 2.11 shows the fetal/infant outcomes according to type of seizure. Infants of mothers with antepartum seizures were more likely to be small for gestational age and to be admitted to a special care baby unit. It should be remembered that there was a close relationship between preterm and antepartum seizures and that the very small numbers prevent meaningful analysis of stratified data. There are therefore limited conclusions to be made from these results.

TABLE 2.10. Pilot study: Outcomes of singletons by gestational age at onset of eclamptic seizure.

\begin{tabular}{|c|c|c|c|c|c|}
\hline \multirow[t]{2}{*}{ Outcome } & \multicolumn{2}{|c|}{$\begin{array}{l}\text { Pre-term }(<37 \text { weeks }) \\
\qquad(\mathrm{n}=20)\end{array}$} & \multicolumn{2}{|c|}{ 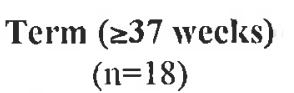 } & \multirow{2}{*}{$\begin{array}{c}\text { Statistical test } \\
\text { Chi square Yates corrected } \\
\text { Relative risk }(95 \% \mathrm{CI}) \\
\end{array}$} \\
\hline & No. & $\%$ & No. & $\%$ & \\
\hline IUGR $(<10$ th centile) & 11 & 55 & 4 & 22 & $\begin{array}{c}3.0 \mathrm{P}=0.08 \\
2.47(0.96-6.41)\end{array}$ \\
\hline Admission to SCBU & 19 & 95 & 7 & $* 41$ & $\begin{array}{c}10.3 P=0.001 \\
2.31(1.30-4.11)\end{array}$ \\
\hline Fetal/infant death & 1 & 5 & 1 & 6 & $\begin{array}{c}\ddagger \mathrm{P}=0.73 \\
0.90(0.06-13,36)\end{array}$ \\
\hline
\end{tabular}

* missing data in one case $\ddagger$ Fisher exact test 
TABLE 2.11. Pilot study: Outcomes of singletons by type of eclampsia.

\begin{tabular}{|c|c|c|c|c|c|}
\hline \multirow[t]{2}{*}{ Outcome } & \multicolumn{2}{|c|}{$\begin{array}{l}\text { Antepartum } \\
\quad(n=16)\end{array}$} & \multicolumn{2}{|c|}{$\begin{array}{l}\text { Intra or Postpartum } \\
\qquad(\mathrm{n}=22)\end{array}$} & \multirow{2}{*}{$\begin{array}{c}\text { Statistical test } \\
\text { Chi square Yates corrected } \\
\text { Relative risk }(95 \% \mathrm{CI})\end{array}$} \\
\hline & No. & $\%$ & No. & $\%$ & \\
\hline IUGR ( $<10$ th centile) & 7 & 44 & 8 & 36 & $\begin{array}{c}0.02 \mathrm{P}=0.65 \\
1.20(0.55-2.63)\end{array}$ \\
\hline Admission to SCBU & 16 & 100 & $* \uparrow 10$ & 53 & $\begin{array}{c}\$ \mathrm{P}<0.001 \\
1.90(1.24-2.91)\end{array}$ \\
\hline Fetal/infant death & 0 & 0 & 2 & 9 & $f \mathrm{P}=0.33$ \\
\hline
\end{tabular}

* missing data in one case $\uparrow$ two babes stillborn and therefore excluded $\$$ Fisher exact test.

\subsection{DISCUSSION.}

The pilot showed that a national study was feasible. Obstetricians and midwives willingly participated in providing notifications and the information obtained from case-note review was more complete than expected. Several areas of the methodology were identified where improvements were needed before implementing a national study. The clinical results of the pilot raised several questions that warranted closer examination in the main study.

There was no external source with which to compare the number of notifications received and check if the ascertainment of cases had been complete. However at least one reply was received from every hospital during the year and there was a tendency to over notify. For these reasons so it was felt that it was unlikely there were large gaps in notifications.

The incidence of eclampsia found in the series was similar to that found in a recent epidemiological study in the United States of America (incidence of eclampsia 4.3/10,000 in 1983-86) (Saftlas et al. 1990). Given there are approximately 780,000 deliveries per year in the United Kingdom (OPCS, 1993), and extrapolating from the incidence in the pilot study, it was estimated that there would be between 300 and 400 cases in the whole of the United Kingdom during 1992.

The reduction in maternal deaths from pre-eclampsia and eclampsia is commonly attributed to the advent of universal antenatal care. However in this series $52 \%$ of the women developed eclampsia despite receiving antenatal care consistent with the traditional pattern. The high proportion (78\%) of first seizures occurring in hospital is consistent with other series from developed areas (Moller \& Lindmark, 1986; Templeton \& Campbell, 1979). It is a little disquieting that more seizures were not prevented once women were hospitalised, but may indicate that many eclamptic seizures occurred unheralded. In fact $39 \%$ of women 
in this series did not have recorded antenatal pre-eclampsia before the first seizure. Sibai found $17 \%$ of women had unheralded seizures (i.e. very rapid onset of pre-eclampsia or very mild pre-eclampsia with controlled hypertension) (Sibai et al. 1986a).

The complication rate in this series was very high; $(56 \%)$. This is considerably higher than the morbidity rates published in other series. A study from Sweden found 15\% of eclamptic women had a major complication (Moller \& Lindmark, 1986), and a recent study from Nigeria found major complications in $31 \%$ of eclamptic women (Adetoro, 1990). The types of complications and morbidity recorded in the Swedish study are very similar to those in this study so the reasons for the difference in complication rate are unclear.

Women with antepartum eclampsia fared less well than women whose eclampsia occurred after the start of labour. Antepartum eclampsia may be a severe variant of disease but there may be other factors that explain the poorer outcome. Many women in the antepartum group had their first seizure at home and hence had a delay between seizure and initiation of management. A second possible explanation is the higher proportion of preterm deliveries in the antepartum group. Preterm deliveries have a high incidence of general anaesthesia and caesarean sections both of which have inherent risks of complications. A larger study such as the proposed national study would be needed to examine these issues in greater detail.

There were several issues raised in the clinical analysis of the pilot which deserved elucidation in the national study.

1. Are there real differences between antepartum and intra or postpartum eclampsia and/or between pre-term and term onset eclampsia?

2. How common is it to find that women with eclampsia have had little or no antenatal care?

3. What proportion of women with eclampsia have their first seizure before developing the classic signs of hypertension and proteinuria?

4. Is the high rate of complications found in the pilot study duplicated in the national study, and if so is there any indication of why there is such a high complication rate?

5 . How commonly are the recommended investigations of liver function actually carried out in women with eclampsia? 


\subsection{LESSONS FROM THE PILOT STUDY ON METHODOLOGY.}

One of the major aims of the pilot study was to test and refine the methods of notification and data collection that were to be used in the main study. During the conduct of the pilot several areas were identified where improvements could be made.

\subsubsection{Requests for notifications.}

The response rate to requests for notifications was markedly improved by sending a reminder letter to those who had not responded with in six weeks. To maximise responses and minimise missed cases reminder letters were adopted for all mail-outs in the national study.

Midwives were consistently better respondents to the requests for notifications than consultants. However in the main study consultants continued to be approached for notifications. This was because multiple letters sent to a range of people seemed necessary to ensure at least one response from every hospital and because the requests provided a simple system for keeping the obstetricians informed about (and therefore interested in), the study.

\subsubsection{Criteria for notifications.}

Having broad criteria for notification of cases worked well; there were 120 notifications received and only 41 included in the analysis. The good response rate and high number of "over" notifications implied that all possible cases of eclampsia had been included. However, a similar rate of notifications during the national study would have been overwhelming with precious time wasted chasing and reviewing cases that were obviously not eclampsia. To prevent this the criteria for notifications in the main study were changed the aim being still to keep the criteria broad but to reduce the number of notifications of cases that were definitely not eclampsia.

\subsubsection{Collection of clinical data.}

\subsubsection{Photocopies of case-notes}

Ninety-nine percent of the notified cases in the pilot study were reviewed but this had required travel to individual hospitals to see the case-notes. To ensure the incidence derived from the national study was accurate it was essential to review all notified cases. However personnel and time restraints meant it would not be possible to travel to individual hospitals to complete the expected 500-600 case-note reviews. It was therefore decided to 
obtain complete photocopies of the case-notes and have them sent to the John Radcliffe Hospital in Oxford were the study was based.

The photocopies of case-notes solved one problem but created several others. In the pilot study the information retrieval was good (i.e. there were only small numbers of missing values in most variables). The concern was that photocopied notes may not be complete or of a sufficiently high quality to allow such complete data retrieval. It was also important to maintain confidentiality and this would be difficult if case-notes were to be sent through the mail. The final hurdle was to motivate people to complete the dull and time consuming job of actually photocopying the case-notes. All of these issues were solved and the details are discussed in the Chapter 3.

\subsubsection{Changes to the data collected.}

In the main study the data sheet was altered to include recordings of the maximum blood pressures before and after the eclamptic seizures so that the change in blood pressures could be analysed. Other changes to the data collected in the main study were the ability to record more than one form of anaesthesia, the inclusion of values of serum urea (as well as uric acid) and records of whether the women had required blood transfusion.

\subsubsection{Reviewing of secondary case-notes.}

During the pilot secondary case-notes were not reviewed and thus valuable information about post eclamptic progress may have remained undiscovered. In the main study all casenotes pertaining to the eclamptic episode were vigorously pursued.

\subsubsection{General practitioner questionnaire.}

The return rate to a single request for information from the general practitioners was $64 \%$. A return rate of at least $75 \%$ was aimed for during the main study and up to four reminder requests were sent in order to achieve this. The general practitioner questionnaire was altered to include questions on the neonate in an effort to improve the information about neonatal outcomes.

\subsubsection{Data extraction and entry onto the computer.}

There were a large number of errors in the pilot data base. Many of these arose during the repeated processing of the data. To reduce these errors the data collection sheet was altered so that it included the appropriate numerical and logical transformations. Information was extracted directly from the case-notes into numerical or logical form which 
was then entered onto the computer. The data were handled less often which meant there was less opportunity for errors. Data were entered onto the computer twice and the two databases compared for inconsistencies which, if found, were corrected.

\subsection{SUMMARY AND CONCLUSIONS.}

The response from obstetricians and midwives to requests for notifications in the pilot study as well as the high quality of data available from case-note review showed that a national study was feasible. It was likely that a national study would include between 300 and 400 women with eclampsia.

The pilot study confirmed that although eclampsia is rare it has a high case fatality rate and very significant rates of complications. Many women in this series developed eclampsia despite receiving full antenatal care and being in hospital. The study showed current recommendations on the investigation of women with eclampsia were not routinely followed. The numbers in the pilot study were small but indicated that eclampsia occurring before the onset of labour or before 37 completed weeks of pregnancy may have more severe consequences for the mother and neonate.

A national study was desirable to develop a better understanding of eclampsia. The larger numbers in a UK wide study would allow elucidation of variants of eclampsia that were particularly severe or some sub-groups of the population who were particularly vulnerable to its effects. Adaptations in the design and conduct of the study were made and applied to the protocol for a national study on eclampsia. 


\section{CHAPTER 3.}

\section{METHODOLOGY}




\subsection{INTRODUCTION.}

The decision to establish a national study of eclampsia was made in October of 1990 and the study was planned for the calendar year of 1992 . The study was funded by the Medical Audit Unit of the Royal College of Obstetricians and Gynaecologists (RCOG), as one of its first national initiatives. The fifteen months prior to the start of the study were spent gathering support from professional associations, publicising the study, obtaining ethics committee approval as well as conducting the pilot discussed in the previous chapter.

\subsection{SETTING UP.}

\subsubsection{Support from professional bodies.}

This study relied on obstetricians, midwives and general practitioners for help and information in the form of notifications, photocopies of case-notes and completed questionnaires. The study had to have formal support of respected professional organisations to give it a "corporate image" and to help establish the momentum of a national collaborative study.

The preliminary protocol was reviewed by the RCOG, the Royal College of Midwives (RCM), the Royal College of General Practitioners (RCGP) as well as the General Medical Services Committee of the British Medical Association. They were asked to comment on the protocol and suggest improvements. All four professional bodies supported the aims of study. They required reassurance that the study design incorporated adequate safeguards for patient confidentiality and that participation required a minimal commitment from their members. Other suggestions were to ensure that all obstetricians and midwives had information about the study before the starting date, to send multiple reminders to encourage continued support and to provide feedback to all participants. These suggestions were incorporated in a revised protocol and formal approval was then granted by all four professional associations.

\subsubsection{Ethics committee approval.}

In 1990-92 there was increasing debate about the ethics of observational studies without informed consent (Anonymous, 1990; Knox, 1992) and it was therefore felt to be important that all local research ethics committees were informed of the study.

The local research ethics committees (LREC) in the United Kingdom are independent bodies with diverse membership. Each committee concerns itself with the research in a small number of hospitals usually based on a NHS district (although in some areas each 
hospital has its own committee). There is no uniformity of working arrangements or application forms between committees and no national committee to give conditional approval to multi-centre trials (Moodie, 1992). There is not even a comprehensive list of the ethics committees or their addresses. Therefore the study involved the daunting task of individually identifying and approaching every ethics committee in the United Kingdom.

Each of the 15 Regional Medical Officers in England, the Directors of Public Health in each of the 15 Scottish Health Boards, the Chief Administrative Medical Officers in each of the 9 Welsh Health Authorities and the 4 Health and Social Services Boards of Northern Ireland were sent multiple copies of the study protocol and a covering letter. They were asked to distribute the protocol and letter to each LREC in their region/authority or board. If they were unwilling to do so they were asked for a list so the LREC's could be approached directly. Overall the response was disappointing.

The LREC's were also approached through the RCOG tutors in each NHS district. Each tutor was asked to forward the study protocol and covering letter to the local ethics committee. This was possible because the study was "RCOG approved" and obtained a better response from both the tutors and the LREC's. However, as there was no central list of all LREC's it was not possible to check that approval had been sought from each one. The final approach was therefore on an ad hoc basis; each time there was direct correspondence with a new hospital a check was made that the local ethics committee had been informed of the study and if it had not then they were immediately sent a copy of the protocol.

The study involved retrospective data collection only. The conduct of the survey did not affect the management of the women in any way. No contact was made with the women and standard data protection methods were used to ensure confidentiality. Most responses from LREC's confirmed that formal approval was not required for an observational study that did not involve patient contact. A few LREC's requested that the study protocol was submitted to formal review and this was done. In all hospitals and districts where formal approval was sought it was received.

The last LREC to grant approval did so in March of 1992 three months into the study and nearly 15 months after the first approval was granted. The idea of a national ethics committee to monitor all multi-centre trials and provide guidance to LREC's about their suitability holds great appeal. 


\subsubsection{Establishing consultant lists and midwife contacts.}

There was no comprehensive, up to date list of obstetricians working in the United Kingdom. The National Perinatal Epidemiology Unit (NPEU) established the most recent and reliable list of obstetricians and gynaecologists in 1991. The NPEU list was compared with the list of consultants from the Royal College of Obstetricians and Gynaecologists and then further cross checked with the Directory of Emergency and Special Care Units (Anonymous, 1991). In this manner a list of over 1100 consultants was established at the start of the study.

The consultant list was updated continuously throughout 1992 to account for new appointments and retirements. By the end of the study regular requests were sent to only 801 consultants. The reduction in numbers was due to the removal of consultants who stated they were no longer active in obstetrics and because some hospitals nominated a single consultant to liaise with the study.

The Royal College of Midwives did have an up to date list of the Directors of Midwifery in every specialist obstetric unit in the United Kingdom. This list was cross checked with the names listed in the Directory of Emergency and Special Care Units (Anonymous, 1991). The director of each unit was approached and asked to identify a senior midwife who would be willing to act as a liaison with the study. This resulted in a list of 279 midwives (one from each obstetric unit) who participated in the study, which was continually updated to take into account resignations or unit closures during 1992.

Each intensive care unit in the United Kingdom was identified from the Directory of Emergency and Special Care Units (Anonymous, 1991) and was provided with protocols, posters and was asked to notify cases of eclampsia. This was done to provide another source of notifications in the unlikely event that a women with eclampsia bypassed the obstetric unit completely.

\subsubsection{Publicity and posters.}

The study was well publicised before it began so that cases of eclampsia which occurred early in the year were not missed. At various times throughout the year there was continued publicity to re-motivate people when their interest in the study had started to lag.

Information and publicity posters were sent to all consultant obstetricians and to participating midwives in November 1991. They were requested to display them in each antenatal or postnatal ward and in the delivery suite of hospitals. 
The Medical Audit Unit of the RCOG publicised the study in the letters they sent regularly to all RCOG members. This began in November 1991 and was subsequently repeated at three monthly intervals. The president of the RCOG recommended participation with the study in his "Letter from the President" in January of 1992. The editorial board of the British Journal of Obstetrics and Gynaecology published a commentary on the need for a national study of eclampsia in May 1992 (Douglas \& Redman, 1992). A series of talks were given to midwifery groups and individual hospitals to explain the aims of the study and encourage participation. All these were planned to help raised awareness of the study and continue high levels of participation. As a complement to these formal publicity measures participants also were given information on the progress of the study in regular mail-outs during the year.

\subsection{COLLECTING NOTIFICATIONS.}

Notifications of eclampsia were sought by regular requests using a system almost identical to that used in the pilot study (Section 2.2.2.).

\subsubsection{Criteria for notifications.}

During the calendar year of 1992 participating midwives and consultants were asked to notify any case of eclampsia, possible eclampsia or unexplained seizure occurring antenatally, intrapartum or in the first 10 post-partum days. They were encouraged to notify all cases they had heard of whether or not they were involved with the woman's care and even if the woman had been transferred from another hospital.

\subsubsection{Regular mail-outs and reminders.}

On the first of January 1992 every consultant and midwife was sent a letter stating that the study had begun. They were requested to notify any case of eclampsia they saw by using the free-post address or the forms at the bottom of the posters sent to them in November.

Regular requests for notifications were then sent on April $3^{\text {rd }}$, July $3^{\text {rd }}$, October $2^{\text {nd }}$ and December $31^{\text {st }} 1992$. The requests were sent to all participating obstetricians and midwives and consisted of notification forms, a reminder of notification criteria and a covering letter. A reply was requested regardless of whether a case was notified. A record was kept of all responses from individuals and collective responses from hospitals. If a reply had not been received within six weeks of the original request then a reminder notification was sent. Reminders mail-outs were sent on May $15^{\text {th }}$, August $8^{\text {th }}$, November $13^{\text {th }} 1992$ and on February $11^{\text {th }} 1993$. 
If respondents had not seen any women with eclampsia then they simply marked the "No" box and returned the form. If they did have a case to report then they filled out the notification form in full. This involved providing the hospital name, the woman's name, her hospital number, the consultant in charge of her care and her general practitioner's name and address. At the bottom of the form the respondents were asked to provide the name and telephone number of someone who was willing to photocopy the case-notes.

\subsubsection{Checking with consultants.}

When a notification was received from a midwife the consultant concerned was contacted by telephone to inform him/her and to double-check on the suitability of the notification. This procedure was instituted at the request of the Royal College of Obstetricians. On the whole, when approached, the consultants felt this double checking was unnecessary and many requested that future notifications from midwives be acted upon without confirmation.

\subsection{VERIFICATION WITH EXISTING SOURCES.}

Validation of the notification system and checks on the completeness of ascertainment were difficult because no national data on eclampsia incidence exist. There are however a few well established, comprehensive maternity data collection systems in individual regions or areas. Where-ever possible the accuracy of the notification system was checked by comparing the number of cases reported with the numbers recorded in these systems.

The notification systems used for comparisons were the Cardiff Birth Survey, the North West Thames Regional database and Scottish Information and Statistics Division maternity data. The number of maternal deaths in the study were compared with the number associated with eclampsia in the Department of Health's register of maternal deaths.

\subsection{COLLECTION OF CLINICAL DATA.}

Each notification was checked to ensure it was not a duplicate and, if necessary, confirmed with the consultant in charge of the case. Each new case notified was allocated a study number and entered into the confidential study register. Arrangements were made to obtain a photocopy of the woman's hospital case-notes. This differed from the system used in the pilot study for the reasons that are discussed in Section 2.5.3.1. 


\subsubsection{Photocopies of case-notes and blinding of data.}

The person listed on the notification form as willing to provide a photocopy of case-notes was contacted and given detailed instructions. A $£ 20.00$ payment was made for each set of complete case-notes received. The payment was enough to cover photocopying and postage costs $(\approx £ 6.00)$ and leave a small gratuity.

The completed photocopies were sent by registered delivery. The blinding of notes was important so that the patient's identity would remain confidential even if the registered mail was lost. The notes were blinded by placing a "post-it" note which had the woman's study number written on it over every single patient identification label in the case-notes (including history and examination sheets as well as all biochemical and haematology results). Thus the case-notes received were identified only by the study number which appeared on every page. The photocopying system worked better than expected. The copies were generally complete and of high quality.

If case-notes were not received within 6 weeks reminders were sent by mail and, if necessary, by repeated telephone calls. All case-notes were vigorously pursued. There were occasional delays but usually in complicated cases where the woman had a particularly stormy course and was being followed up by several doctors and/or lawyers. In these cases patience persistence eventually proved fruitful.

Once the photocopied case-notes were received a detailed case-note review was completed. If the woman did not meet the criteria for eclampsia then a note was made of the actual or probable diagnosis but detailed information was not recorded.

\subsubsection{Criteria for inclusion in eclampsia series.}

Women were included in the eclampsia series under two categories, "classical" eclampsia and "probable" eclampsia.

For the purposes of the study "classical eclampsia" was defined as the occurrence of seizures during pregnancy or in the first 10 days post-partum when the patient had at least two of the following characteristics within 24 hours before or after the seizures: hypertension, proteinuria, thrombocytopaenia and/or elevated aspartate transaminase level.

Hypertension was defined as a booking diastolic pressure of $<90 \mathrm{~mm} \mathrm{Hg}$, a maximum diastolic of $\geq 90 \mathrm{~mm} \mathrm{Hg}$ and a diastolic increment of $\geq 25 \mathrm{~mm} \mathrm{Hg}$.

Proteinuria was defined as $2+$ of protein in a random sample or $\geq 0.5$ grams in a 24 hour collection. 
Thrombocytopenia was defined as a platelet count of less than $100 \times 10^{9} /$.

A raised plasma level of aspartate transaminase (AST) was defined as $\geq 42$ IU.

Other cases were included as "probable eclampsia" if;

a. As with classical eclampsia except that $1+$ of proteinuria or $\geq 0.3$ grams in a 24 hour collection was accepted as significant.

b. They met the criteria for inclusion as "classical" eclampsia but there was another complicating factor in the diagnosis. Examples of these were women with clear proteinuric pre-eclampsia who had a seizure but who also had a personal history of epilepsy.

Cases in this second category of probable eclampsia often proved a diagnostic dilemma and were all discussed with Professor Redman prior to inclusion in the series. A woman was not included if she had recurrent epileptic seizures throughout pregnancy, but was included if the epilepsy had been well controlled and quiescent in the pregnancy until she developed pre-eclampsia. Other examples of women included in the probable category were women who were found in a post-ictal state, had clear proteinuric pre-eclampsia but in whom a seizure had been presumed but not actually witnessed.

\subsubsection{Hospital case-note data.}

If the criteria for eclampsia were met detailed data were extracted from the copy of the case-notes onto a pre-prepared data sheet To ensure maximum data retrieval information was gathered from the doctors', midwifery and intensive care notes as well as from observation charts, delivery and anaesthetic records.

The data were almost identical to those collected in the pilot study with some additions and refinements (Section 2.5.3.2). The data covered 8 areas: completeness of information, social and demographic factors, antenatal care, prodromal signs and symptoms, features of eclampsia, features of delivery, maternal outcome and fetal outcome.

The data sheet had each of the variables and their definitions printed on it as well as the appropriate numerical or logical transformations. Data were extracted directly onto the sheet in the form in which it would be entered onto the computerised data base thus minimising data handling.

If, on review of the index case-notes, it was found that the woman had been transferred to or from another hospital then a photocopy of those secondary case-notes were also requested. Missing data and case-notes were vigorously pursued by repeated letters and telephone calls. 


\subsubsection{General Practitioner Questionnaires.}

In all cases of eclampsia the woman's general practitioner (GP) was sent a questionnaire which asked for details about shared antenatal care, maternal puerperal morbidity and neonatal outcome. Post-eclamptic morbidity was assessed by asking GP's if they had seen the woman and if any of 16 listed factors had been an issue during consultation.

The general practitioner questionnaires were sent out as soon as the hospital case-notes were received. If there was no reply within 4 weeks then a reminder questionnaire was sent and this was repeated up to four times. If women changed GP's after the eclamptic episode the new GP was traced through the Family Practitioner Committees.

\subsubsection{Collection of extra baby information.}

Efforts were made to improve the information on neonates that had been admitted to a Special Care Baby Unit (SCBU) and retained in hospital after the mothers discharge. In many cases the information was available from the general practitioner questionnaire but in cases where the general practitioner failed to respond the SCBU was approached directly for information about neonatal morbidity and mortality.

\subsubsection{Definitions of terms used in the study.}

The definition for eclampsia itself is given in Section 3.5.2.

Antenatal care was assessed relative to a traditional pattern of a booking visit before 20 weeks gestation, monthly visits until 30 weeks, fortnightly visits until 36 weeks and weekly visits thereafter. Care that did not conform to this pattern did not necessarily imply substandard care.

The gestational age at the time of seizure was recorded and for postpartum seizures this was taken to be the gestational age at delivery.

Eclampsia was defined as unheralded if the woman had a urine screen and blood pressure measurement in the 24 hours preceeding the onset of seizures and did not have established proteinuria and hypertension, although these signs then had to be documented in the 24 hours following the seizure.

Maternal complications were defined as those recorded in hospital case-notes, and were classified as major (e.g. disseminated intravascular coagulopathy, renal failure, 
cerebrovascular accident) or minor (e.g. urinary tract infection or lower respiratory tract infection).

A maternity denotes a pregnancy which resulted in a stillbirth or live birth. A stillbirth refers to a child born after the twenty-fourth week of pregnancy without any sign of life.

Fetuses were classified as small for gestational age if their birth weights were less than the tenth centile for their gestational age using charts based on Oxford data (Yudkin et al. 1987).

\subsection{ESTABLISHMENT OF DATABASE.}

The study data base was established using the software programme DBASE IV.1. The data-base consisted of all variables shown on the data sheet (see Appendix). Women who were notified but did not have eclampsia had their study number, the region and the probable diagnosis recorded but no other information.

All variables for cases of eclampsia were entered into the data base which was established with range-checks to limit the number of keying-in errors. Data keyed in that were outside these ranges would not be accepted and were manually checked with the photocopied casenotes.

All data were then entered by a different person onto a second identical database under a different file name. The two files were compared using EPI-INFO's Validate programme (Dean et al. 1990). Any inconsistencies were flagged and double checked with the original data sheets and corrected.

Several internal checks were also performed to find other errors in the data base. For example the age of the woman recorded was cross checked by subtracting the date of birth from the date of delivery, dividing by 365 and the results compared.

Once the data were entered using range checking, had been double keyed, validated and internally checked it was accepted for analysis. Never-the-less occasional inconsistencies or errors were found during the subsequent analyses. These were all checked with the original data sheets and photocopied case-notes.

\subsection{DENOMINATOR DATA.}

One of the advantages of a national study was that United Kingdom maternity statistics could be used for denominator data. The Office of Population Censuses and Surveys (OPCS) (covering England and Wales), the General Registrar Office in Scotland and the 
Registrar General in Northern Ireland were all approached for national data on the following variables: age, country of birth, marital status, social class, previous pregnancies and births, month of delivery, location of delivery, induction of labour, method of delivery, fetal outcome, sex of baby.

All this information was not available from all four constituent countries. The OPCS could provide most of the data but in some cases the definitions used in England and Wales were different to those used in Scotland. Another problems which should have been foreseen was that parity data from the OPCS included women with previous live births only. The study data had variables for previous viable (live and stillbirths) and non-viable (miscarriages and termination's) pregnancies but it was not possible to extract only those women with previous live births.

It was eventually possible to obtain the number of maternities, the number of singleton and multiple pregnancies, and the number of still- and live births and the maternal age at delivery for all four countries.

\subsection{ANALYSIS.}

\subsubsection{General approach.}

The first stage of the analysis produced descriptive information on eclampsia in the United Kingdom. The next step included the use of denominator data to put eclampsia into the context of the whole maternity population. The final stage looked at particular subgroups to assess if there were significant differences within or between the groups.

\subsubsection{Statistical methods.}

Two computerised statistical packages SPSS for Windows and EPI-INFO (Dean et al. 1990) were used to analyse the data.

Means and standard deviations (SD) were calculated for continuous variables. Groups of continuous variables were compared using Kruskal Wallis test for two groups. Confidence intervals for rates were calculated. Further statistical analyses utilised the chi square test with Yates correction, the Fisher exact test and the chi square test for trend where appropriate (with one degree of freedom unless otherwise stated). Relative risks and the associated $95 \%$ confidence intervals were also calculated. For stratified analyses the Mantel-Haenszel summary chi square and summary relative risks were computed. 


\subsubsection{Multiple regression analysis}

Later analysis involved multiple logistic regression which was conducted using the advanced statistical module of SPSS for Windows. The statistical technique used in each of the 4 analyses was the same and is outlined below.

The dependent variables analysed were of four different types; binary, categorical, continuous and interaction variables. For categorical variables the indicator contrast was used with the first category as reference category. All the continuous variables tested (i.e. the various blood pressure measurements) had a range of values with the minimum being considerably greater than zero. These variables were therefore adjusted prior to entry into the regression analysis to prevent the estimate of an extreme odds ratio at the zero point. The adjustment for each variable was accomplished by subtracting the median value for the group as a whole (i.e. adjusted maximum diastolic blood pressure = maximum diastolic blood pressure - median (max. diastolic blood pressure)) (Attewell, unpublished).

Interaction variables were included to see if there was evidence of effect modification between the two variables. i.e. the interaction term gestation of fit $\mathrm{x}$ type of fit was included because it was felt that preterm infants may have been more affected by antepartum seizures than term infants.

Forward stepwise logistic regression was carried out. Removal testing was based on the probability of the likelihood ratio statistic (which is based on the maximum partial likelihood estimates). The probability level for entry into the model was 0.05 and for removal was 0.10. The constant was included in all models.

\subsection{SUMMARY.}

The study was conducted with the financial support of the Medical Audit Unit of the RCOG. Important political and practical support was also given by the RCM, RCGP and the General Medical Services Council of the British Medical association. Every local ethics committee in the United Kingdom was informed of the study and formal approval was obtained whenever it was deemed necessary.

Notifications were received via regular mail-outs to obstetricians and midwives. A blinded photocopy of all hospital case-notes pertaining to eclampsia was obtained and a case-note review was completed. In addition a questionnaire was completed by the women's GP. The methodology used was designed to maximise notifications and ensure complete data collection. The handling of data was streamlined by use of computerised databases and 
multiple checking systems were instituted. The analysis of the data was carried out in three phases with the aid of SPSS for Windows and EPI-INFO V. 


\section{CHAPTER 4.}

\section{DESCRIPTIVE ANALYSIS RESULTS AND DISCUSSION 1}




\subsection{NOTIFICATION AND DATA COLLECTION.}

\subsubsection{Response rates to requests for notifications. \\ 4.1,1.1. From consultants and midwives.}

The response rate to requests for notifications was $90 \%$ from midwives and $70 \%$ from consultant obstetricians. Responses from midwives were uniformly good throughout the whole of the United Kingdom (81\%-99\%) (Table 4.1.) and for the whole of the year (88\%91\%) (Table 4.2.). Responses from obstetricians varied substantially between regions (53\% to $82 \%$ ) (Table 4.1.) but were consistent throughout the year (69\%-72\%) (Table 4.2.).

\subsubsection{From hospitals.}

A hospital was considered to have responded if at least one reply from either a consultant or midwife had been received. The hospital response rate to individual requests for notifications was between 99\% (275/279) and 99.6\% (278/279). Every hospital (100\% of 279 ) in the United Kingdom with a consultant obstetric unit replied to at least 3 of the 4 requests for notifications during 1992. In 95\% (266/279) of the hospitals at least one reply was received in response to each of the 4 requests for notifications (Table 4.2.).

\subsubsection{Degree of over notification.}

During the study 920 positive notification forms were received detailing a total of 582 different cases. Two-hundred and twenty-six (39\%) of all cases were notified more than once.

Of the 582 cases that were notified 198 were excluded from the series of eclamptic women. (see Figure 4.1.). Sixty cases notified by midwives were deemed inappropriate by the consultants when checked and 138 cases were excluded after case-note reviews. The excluded cases included; 73 women with pre-eclampsia and no seizure, 56 with uncomplicated epilepsy, 40 with simple faints, hypoglycaemic fits or pseudo-seizures and 29 women where no cause for the seizure was found but criteria for inclusion in the eclampsia case series were not met. 
TABLE 4.1. The overall response rates to requests for notifications by regions.

\begin{tabular}{|c|c|c|c|c|c|c|}
\hline \multirow[t]{2}{*}{ Area } & \multicolumn{2}{|c|}{ Consultants } & \multicolumn{2}{|c|}{ Midwives } & \multicolumn{2}{|c|}{ Hospitals* } \\
\hline & No. & $\%$ & No. & $\%$ & No. & $\%$ \\
\hline United Kingdom & $2254 / 3204$ & 70 & $1001 / 1116$ & 90 & $1103 / 1116$ & 99 \\
\hline England & $1722 / 2504$ & 69 & $790 / 880$ & 90 & $868 / 880$ & 99 \\
\hline Northern Ireland & $111 / 140$ & 79 & $63 / 72$ & 88 & $72 / 72$ & 100 \\
\hline Scotland & $261 / 360$ & 73 & $93 / 100$ & 93 & $100 / 100$ & 100 \\
\hline Wales & $160 / 200$ & 80 & $52 / 64$ & 81 & $63 / 64$ & 98 \\
\hline \multicolumn{7}{|l|}{ English N.H.S. } \\
\hline East Anglia & $98 / 120$ & 82 & $35 / 36$ & 97 & $35 / 36$ & 97 \\
\hline Mersey & $88 / 128$ & 69 & $37 / 44$ & 84 & $43 / 44$ & 98 \\
\hline North-East Thames & $164 / 252$ & 65 & $57 / 68$ & 84 & $66 / 68$ & 97 \\
\hline North-West Thames & $134 / 196$ & 68 & $70 / 72$ & 97 & $72 / 72$ & 100 \\
\hline North Western & $135 / 204$ & 66 & $71 / 80$ & 89 & $77 / 80$ & 96 \\
\hline Northern & $147 / 188$ & 78 & $76 / 80$ & 95 & $80 / 80$ & 100 \\
\hline Oxford & $65 / 88$ & 74 & $38 / 40$ & 95 & $40 / 40$ & 100 \\
\hline South-East Thames & $134 / 228$ & 59 & $61 / 72$ & 85 & $71 / 72$ & 99 \\
\hline South-West Thames & $91 / 172$ & 53 & $48 / 56$ & 86 & $54 / 56$ & 96 \\
\hline South-Western & $94 / 124$ & 76 & $45 / 48$ & 94 & $52 / 52$ & 100 \\
\hline Trent & $152 / 200$ & 76 & $67 / 68$ & 99 & $67 / 68$ & 99 \\
\hline Wessex & $94 / 120$ & 78 & $46 / 52$ & 89 & $51 / 52$ & 98 \\
\hline West Midlands & $184 / 268$ & 69 & $75 / 88$ & 85 & $88 / 88$ & 100 \\
\hline Yorkshire & $148 / 216$ & 69 & $64 / 72$ & 89 & $72 / 72$ & 100 \\
\hline
\end{tabular}

*A hospital was said to have responded if at least one reply was obtained from either a midwife or consultant. N.H.S. National Health Service

\subsubsection{Double notification of eclamptic cases.}

Three hundred and eighty three cases were included in the series of eclampsia and in 189 (49\%) of these two or more notifications of the case had been received. In some instances up to five notifications of the same case were received.

The lower part of Figure 4.1 shows the classification of the 383 cases of eclampsia that were included in the study. 
TABLE 4.2. Responses rates to each request for notifications

\begin{tabular}{|c|c|c|c|c|c|c|}
\hline \multirow[t]{2}{*}{ Request } & \multicolumn{2}{|c|}{$\begin{array}{c}\text { Consultants } \\
(\mathrm{n}=801)\end{array}$} & \multicolumn{2}{|c|}{$\begin{array}{l}\text { Midwives } \\
(\mathrm{n}=279)\end{array}$} & \multicolumn{2}{|c|}{$\begin{array}{l}\text { Hospitals* } \\
(\mathrm{n}=279)\end{array}$} \\
\hline & No. & $\%$ & No. & $\%$ & No. & $\%$ \\
\hline April & 555 & 69 & 255 & 91 & 275 & 99 \\
\hline July & 549 & 69 & 244 & 87 & 275 & 99 \\
\hline October & 576 & 72 & 255 & 91 & 278 & 100 \\
\hline December & 574 & 72 & 247 & 89 & 275 & 99 \\
\hline $\begin{array}{l}\text { Responded to } \\
\text { all } 4 \text { requests }\end{array}$ & 345 & 43 & 216 & 77 & 266 & 95 \\
\hline
\end{tabular}

*A hospital was said to have responded if at least one reply was obtained from either a midwife or consultant.

FIGURE 4.1. Outcome of notifications.

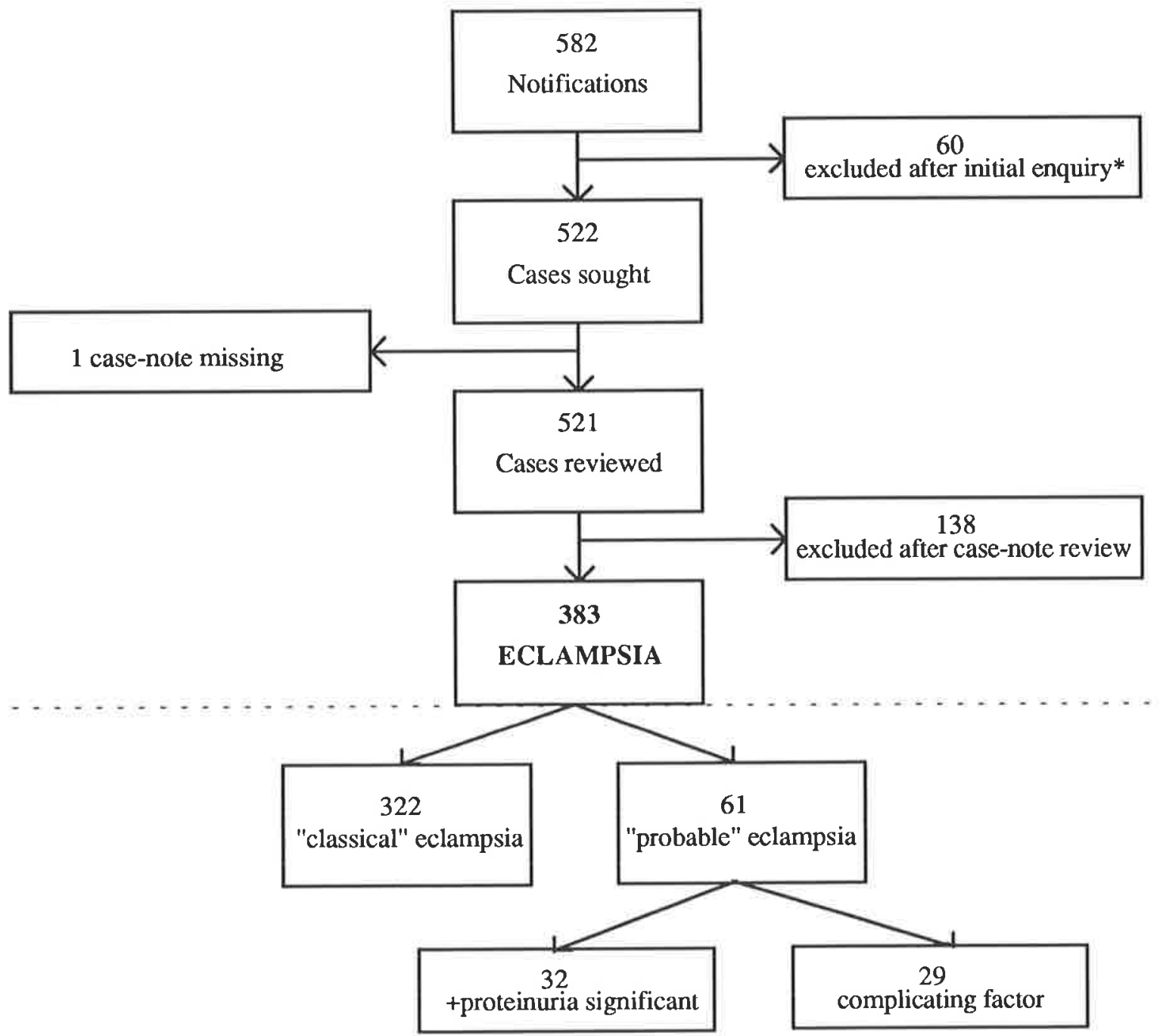




\subsubsection{Comparison with existing maternity data systems.}

To check on the completeness of ascertainment of cases the number of confirmed cases of eclampsia in the study data base was compared with those in existing maternity data information systems in the North West Thames Regional Database, the Cardiff Births Survey and Scottish Information and Statistics Data and with the Department of Health's register of maternal deaths.

For each comparison the number of cases recorded by the established systems were the same or fewer than the number of cases of eclampsia recorded in the study database (details Table 4.3). In fact the study recorded twice the number of cases of eclampsia that the NWT regional data base had recorded and was able to inform the Confidential Enquiries into Maternal Mortality of two deaths they had not previously been notified of.

TABLE 4.3. Validation of notification system by comparison with established maternity data systems.

\begin{tabular}{cccc}
\hline $\begin{array}{c}\text { Established data collection } \\
\text { system }\end{array}$ & $\begin{array}{c}\text { No. of cases in } \\
\text { established system }\end{array}$ & $\begin{array}{c}\text { No. of cases in } \\
\text { study }^{*}\end{array}$ & $\begin{array}{c}\text { Percentage } \\
\text { ascertainment }\end{array}$ \\
\hline Cardiff Birth Survey & 2 & 2 & $100 \%$ \\
Scotland ISD & 28 & 44 & $157 \%$ \\
NWT Regional database & 11 & 21 & $191 \%$ \\
CEMD & $5 \dagger$ & 7 & $140 \%$ \\
\hline
\end{tabular}

* In the same area as covered by each of the established systems. $\dagger$ Provisional figures for 1992 as known by December 1994. ISD-Information and Statistics Division of the National Health Service in Scotland. NWT-North West Thames. CEMD-Confidential Enquiry into Maternal Deaths in the United Kingdom

\subsubsection{Hospital case-notes reviewed.}

Five-hundred and twenty-two cases-notes were sought. There was one case where all trace of the woman and her hospital admission was lost and no information was obtained at all. There were 521 cases where some information was available. In all but one of these (99.8\%) the index hospital case-notes were reviewed. In the case where hospital notes were lost information was retrieved from labour records, the consultant's and midwife's memory and from the general practitioner questionnaire. 


\subsubsection{Quality of information retrieved.}

As with the pilot study the percentage of missing values varied considerably with the type of data. Basic social and demographic data was the most poorly recorded type of information in hospital case-notes. The date of birth was always recorded but marriage status was missing in 17 (4\%), information on occupation and/or social class was missing in $72(19 \%)$ and data on ethnic origins and country of birth were missing in $176(46 \%)$ and 187 (49\%) respectively. In contrast, variables concerning the eclamptic episode had very low percentages $(0.3-2 \%)$ of missing values. Variables concerned with the woman's past medical history were better recorded than the demographic information, but were not as complete as the information directly concerning the eclamptic episode.

\subsubsection{Extra case-notes requested and reviewed.}

There were 73 cases where the woman had been cared for at more than one hospital and in all these cases the secondary hospital case-notes were requested. The secondary case-notes were received and reviewed in 57 cases (78\%).

\subsubsection{General Practitioner Questionnaires received.}

Questionnaires were sent to the general practitioner's of all 383 women included in the eclampsia series. Three-hundred and twenty-six (85\%) of the general practitioner questionnaires were returned at least partially completed.

In $312(81 \%)$ cases all hospital notes (index and secondary) and the general practitioner questionnaire were reviewed.

\subsubsection{Complete antenatal care records.}

In $304(79 \%)$ cases a complete copy of the woman's antenatal care record was obtained from the consultants, hospital case-notes and/or the general practitioners.

In 261 cases (68\%) complete data collection from all sources was achieved as summarised in Figure 4.2. 
FIGURE 4.2. Completeness of data collection.

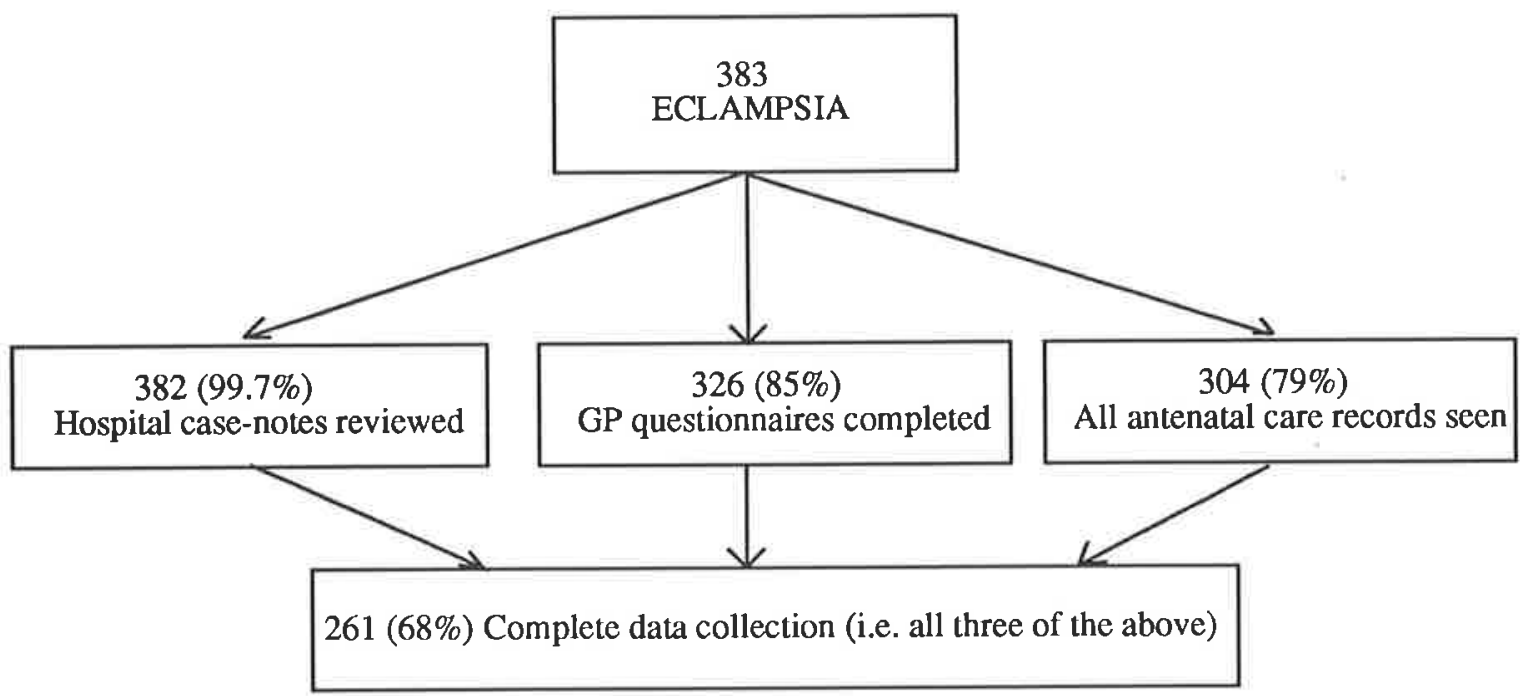

\subsection{DESCRIPTIVE ANALYSIS.}

\subsubsection{Incidence.}

There were 774,436 maternities in the United Kingdom during 1992 and 383 confirmed cases of eclampsia which gives an incidence of 4.9/10,000 maternities (95\% confidence interval 4.5-5.4).

Table 4.4. shows the incidence in the constituent countries of the United Kingdom as well as the Regional Health Authorities of the English National Health System. The incidences in regions varied between $3.4 / 10,000$ in the Oxford Regional Health Authority to 7.6/10,000 in the Northern Regional Health Authority.

\subsubsection{Risk factors.}

\subsubsection{Age.}

The mean age of women in the series was 26.1 (SD 6.0). There were 72 women under the age of 20 years who developed eclampsia in 1992 which represents $19 \%$ of all eclamptic women. Teenagers in the study were three times more likely to suffer eclampsia than older women (relative risk (95\% CI) $3.0(2.4<\mathrm{RR}<4.0)$ ) (Table 4.5.). No increase risk was detected in women over the age of 34 years. 
TABLE 4.4. Incidence of Eclampsia in the United Kingdom, it constituent countries and in the Regional Health Authorities of the English N.H.S.

\begin{tabular}{lcccc}
\hline \multicolumn{1}{c}{ Area } & $\begin{array}{c}\text { No Cases } \\
\text { Eclampsia }\end{array}$ & $\begin{array}{c}\text { No. } \\
\text { Maternities }\end{array}$ & $\begin{array}{c}\text { Rate of } \\
\text { Eclampsia/10,000 } \\
\text { maternities }\end{array}$ & $\begin{array}{c}\text { 95\% Confidence } \\
\text { interval for rate }\end{array}$ \\
\hline United Kingdom & 383 & 774,436 & 4.9 & $(4.5-5.4)$ \\
\hline England & 307 & 646,456 & 4.7 & $(4.2-5.3)$ \\
Northern Ireland & 17 & 25,425 & 6.7 & $(3.5-9.9)$ \\
Scotland & 44 & 65,307 & 6.7 & $(4.6-8.7)$ \\
Wales & 15 & 37,248 & 4.0 & $(2.0-6.1)$ \\
\hline English N.H.S. & & & & $(2.0-7.2)$ \\
East Anglia & 12 & 25,879 & 4.6 & $(2.1-6.8)$ \\
Mersey & 14 & 31,378 & 4.5 & $(3.3-7.0)$ \\
North-East Thames & 29 & 56,465 & 5.1 & $(2.6-6.3)$ \\
North-West Thames & 23 & 51,498 & 4.5 & $(2.3-5.6)$ \\
North Western & 22 & 55,907 & 3.9 & $(4.9-10.3)$ \\
Northern & 30 & 39,709 & 7.6 & $(1.5-5.3)$ \\
Oxford & 12 & 35,731 & 3.4 & $(3.1-7.1)$ \\
South-East Thames & 26 & 50,917 & 5.1 & $(1.9-5.7)$ \\
South-West Thames & 15 & 39,810 & 3.8 & $(2.1-6.0)$ \\
South-Western & 16 & 39,793 & 4.0 & $(2.6-5.8)$ \\
Trent & 26 & 61,727 & 4.2 & $(3.3-8.1)$ \\
Wessex & 21 & 36,892 & 5.7 & $(3.1-7.1)$ \\
West Midlands & 36 & 71,434 & 5.0 & 5.1 \\
Yorkshire & 25 & 49,316 & & \\
\hline & & & & \\
\hline
\end{tabular}

Population data on number of maternities from OPCS. VS2 1992, General Registrars Office Northern Ireland and General Registrars Office Scotland.

\subsubsection{Parity.}

There was a relatively high proportion of primiparae; $286(75 \%)$ in the eclamptic series. National data on number of previous still- and live births does not exist so that accurate calculation of rates of eclampsia in primiparous women could not be done.

Ninety-nine percent (71) of the teenagers were primiparous compared to 69\% (215) of women aged 20 or more. Part of the apparent increased risk associated with low maternal age may be due to primiparity but it is not possible to assess this accurately. There were 4 $(1 \%)$ women who were grand multiparae i.e. 5 or more previous viable pregnancies. 


\subsubsection{Multiple pregnancy.}

Twenty-seven $(7 \%)$ of the maternities were multiple pregnancies; 26 sets of twins and one triplet pregnancy. Women with multiple pregnancies had a significantly higher incidence of eclampsia (Table 4.5.) with a relative risk $(95 \%$ CI) of developing eclampsia of 6.0 $(4.1<\mathrm{RR}<8.9)$ compared to women with singletons.

TABLE 4.5. Incidence rate of eclampsia by maternal age and by multiple pregnancies.

\begin{tabular}{lccc||cc}
\hline & \multicolumn{3}{c||}{ Maternal age (years) } & \multicolumn{2}{c}{ Fetal number } \\
& $\leq \mathbf{1 9}$ & $\mathbf{2 0 - 3 4}$ & $\mathbf{3 5}$ & Multiple & Singleton \\
\hline No Cases Eclampsia & 72 & 278 & 33 & 27 & 356 \\
No. Maternities* & 54,885 & 645,246 & 74,244 & 9,618 & 764,818 \\
Rate of Eclampsia/10,000 & $13.1 \dagger$ & 4.3 & 4.4 & $28.1 \ddagger$ & 4.7 \\
maternities (95\% CI for rate) & $(10.1-16.1)$ & $(3.8-4.8)$ & $(2.9-6.0)$ & $(17.5-38.6)$ & $(4.2-5.1)$ \\
\hline
\end{tabular}

Population data on number of maternities from OPCS. VS2 1992, General Registrars Office Northern Ireland and General Registrars Office Scotland. *OPCS. Population data - the maternal age unknown in 61 cases. $†$ chi square $=79.8$, df $1, p<0.0001$; Relative risk $(95 \% \mathrm{CI}) 3.0(2.4<\mathrm{RR}<4.0)$ of women $<20$ compared to women $\geq 20$. $\ddagger$ chi square with Yates correction $=100.4, p<0.0001$; relative risk $(95 \% \mathrm{CI}) 6.04 .1<\mathrm{RR}<8.9)$ of women with multiple pregnancies compared to those with singleton pregnancies.

\subsubsection{Race.}

Information on the mother's country of birth was available in only 207 (54\%) cases. Of these women $164(79 \%)$ were born in the United Kingdom, $5(2 \%)$ in other European countries, $10(5 \%)$ in India, $16(8 \%)$ in other Asian countries, $8(4 \%)$ in Africa and $4(2 \%)$ were born in other countries. Comparable data for all women giving birth from the whole of the United Kingdom is not available.

Information is available for the country of mother's birth for live births in England and Wales and so data has been extracted for live births from eclamptic pregnancies in England and Wales to allow some form of comparisons (see Table 4.6.). It appears that Asian and African women are over represented in the eclamptic population in comparison to the total population. Due to the high proportion of missing data this finding should not be overinterpreted as there may well have been a bias toward reporting country of birth in women who were obviously not Caucasian. 
TABLE 4.6. Country of birth of mother by live birth from eclamptic pregnancies in England and Wales and by all live births in England and Wales

\begin{tabular}{lrrrr}
\hline $\begin{array}{l}\text { Country of birth of } \\
\text { mother }\end{array}$ & \multicolumn{2}{c}{$\begin{array}{c}\text { Live births eclamptic } \\
\text { pregnancies }(\mathrm{n}=191)\end{array}$} & \multicolumn{2}{c}{$\begin{array}{c}\text { All live births } \\
(\mathbf{n = 6 8 9 6 5 6})^{*}\end{array}$} \\
& No. & \multicolumn{1}{c}{$\%$} & \multicolumn{1}{c}{ No. } & \% \\
\hline United Kingdom & 152 & 79.6 & 607709 & 88.1 \\
Other European & 5 & 2.6 & 16014 & 2.3 \\
Indian & 8 & 4.2 & 7694 & 1.1 \\
Other Asian & 14 & 7.3 & 18347 & 2.7 \\
African & 8 & 4.2 & 11876 & 1.7 \\
Other & 4 & 2.1 & 28016 & 4.1 \\
\hline
\end{tabular}

*Population data from OPCS. Birth Statistics 1992

\subsubsection{Body build.}

The height at booking ranged form 1.35 metres to 1.85 metres, was normally distributed and had a mean (SD) of $1.60(0.70)$ metres. Women's booking weight varied from $38-146$ $\mathrm{kg}$ with a mean (SD) of $62(14.1)$.

The body mass index (weight in $\mathrm{kg} /$ (height in metres) $^{2}$ ) was calculated in $309(81 \%)$ cases. The mean (SD) body mass index was $24.0(5.1) \mathrm{kg} / \mathrm{m}^{2}$ with a range of $15.1-53.6 \mathrm{~kg} / \mathrm{m}^{2}$. The 10 th centile was $19.2 \mathrm{~kg} / \mathrm{m}^{2}$ and the 90 th centile was $29.8 \mathrm{~kg} / \mathrm{m}^{2}$.

\subsubsection{Cigarette smoking.}

Information on smoking at the time of booking was available in $332(87 \%)$ of the women with eclampsia. Two-hundred and forty-six (74\%) of these women did not smoke at all, 59 (18\%) smoked between 1-10 per day and only 27 (8\%) women admitted to smoking more than 10 cigarettes per day at the time of booking.

\subsubsection{Others.}

Twenty-six (27\%) of the 96 women with previous viable pregnancies had a history of preeclampsia and one had a history of eclampsia. Thus $18 \%$ of all the cases involved parous women with no recorded previous history of pre-eclampsia/eclampsia.

Other risk factors documented in the case-notes included: a history of renal disease in 8 (2\%) women, chronic hypertension in $6(2 \%)$ insulin dependent diabetes in $4(1 \%)$ and a history of auto-immune disorder in $2(0.5 \%)$. 


\subsubsection{Antenatal care.}

Thirteen women (3\%) had no antenatal care before the onset of convulsions . Of the remainder, it was possible to review the entire record of antenatal visits in 291 (79\%) cases. Of these, $206(71 \%)$ had antenatal care corresponding to or exceeding the traditional pattern. Eighteen women (6\%) booked after 20 weeks and 67 (23\%) had less than the standard frequency of visits. Characteristics of antenatal care are discussed further in section 5.1.

\subsubsection{Location of patient at time of first seizure.}

Eighty-four (22\%) women had their first seizure prior to admission to hospital and two $(0.5 \%)$ had postpartum seizures after discharge from hospital. A further 131 (34\%) women had been in hospital for less than 24 hours at the time of the first seizure.

Of the 84 women who had their first seizure before admission to hospital a complete record of antenatal care was seen in $74 \%(62 / 84)$. Two thirds of these women $(66 \%-41 / 62)$ received antenatal care corresponding to or exceeding the standard pattern in the United Kingdom. Sixty-nine percent (43/62) of this group had their blood pressure measured and $63 \%(39 / 62)$ had urine checked in the 14 days prior to the onset of seizures. Six $(10 \%)$ of these women had hypertension alone, 11 (18\%) had proteinuria and 4 (7\%) had documented proteinuria and hypertension prior to the onset of seizure but were still cared for in the community.

\subsubsection{Prodromal signs and symptoms.}

Three-hundred and twenty-five $(85 \%)$ women had been seen by a doctor or midwife in the week before their first convulsion. At the time of the last antenatal visit $36(11 \%)$ had no recorded hypertension or proteinuria, $32(10 \%)$ had proteinuria but no hypertension, 71 (22\%) had hypertension alone and only 186 (57\%) women had both proteinuria and hypertension.

Two-hundred and ninety-six $(78 \%)$ women were in hospital when eclampsia first occurred and even in this subgroup only $183(62 \%)$ had established proteinuria and hypertension prior to the first fit.

\subsubsection{Blood pressure.}

Figure 4.3. shows the mean systolic and diastolic blood pressures at intervals throughout the pregnancy. The mean blood pressure at booking (for the 333 women who booked 
before 20 weeks gestation) was $114 \mathrm{~mm} \mathrm{Hg}$ (SD 13) systolic over $68 \mathrm{~mm} \mathrm{Hg}$ (SD 10) diastolic.

The maximum blood pressures before the onset of eclampsia were often not dramatically elevated: of the 296 women who had an eclamptic fit while in hospital, the maximum diastolic blood pressure recorded before onset of seizures was $\leq 110 \mathrm{~mm} \mathrm{Hg}$ in 195 (66\%) women, $\leq 100 \mathrm{~mm} \mathrm{Hg}$ in $100(34 \%)$ women, and $\leq 90 \mathrm{~mm} \mathrm{Hg}$ in $51(17 \%)$ women. In 201 women the blood pressure was measured in the hour before the onset of seizures and the mean blood pressure was $158 \mathrm{~mm} \mathrm{Hg}$ (SD 23) systolic and 97 (SD 14.6) mm Hg diastolic. Very high diastolic blood pressures $(\geq 120 \mathrm{~mm} \mathrm{Hg}$ ) before the onset of seizures were recorded in only $70(19 \%)$ women overall; this represents $23 \%$ (69) of those under hospital care and $2 \%$ (1) of women still receiving care in the community.

The mean maximum blood pressure (measured at anytime in pregnancy or in the hospital admission for eclampsia) was $181 \mathrm{~mm} \mathrm{Hg}$ (SD 23) systolic over $116 \mathrm{~mm} \mathrm{Hg}$ (SD 13) diastolic. The mean change in diastolic blood pressure from booking to the maximum recorded was normally distributed with a mean of $47 \mathrm{~mm} \mathrm{Hg}$ (SD 14).

FIGURE 4.3. Graph showing the mean and standard deviation of the systolic and diastolic blood pressures at booking, immediately before seizure and the maximum recorded blood pressures.

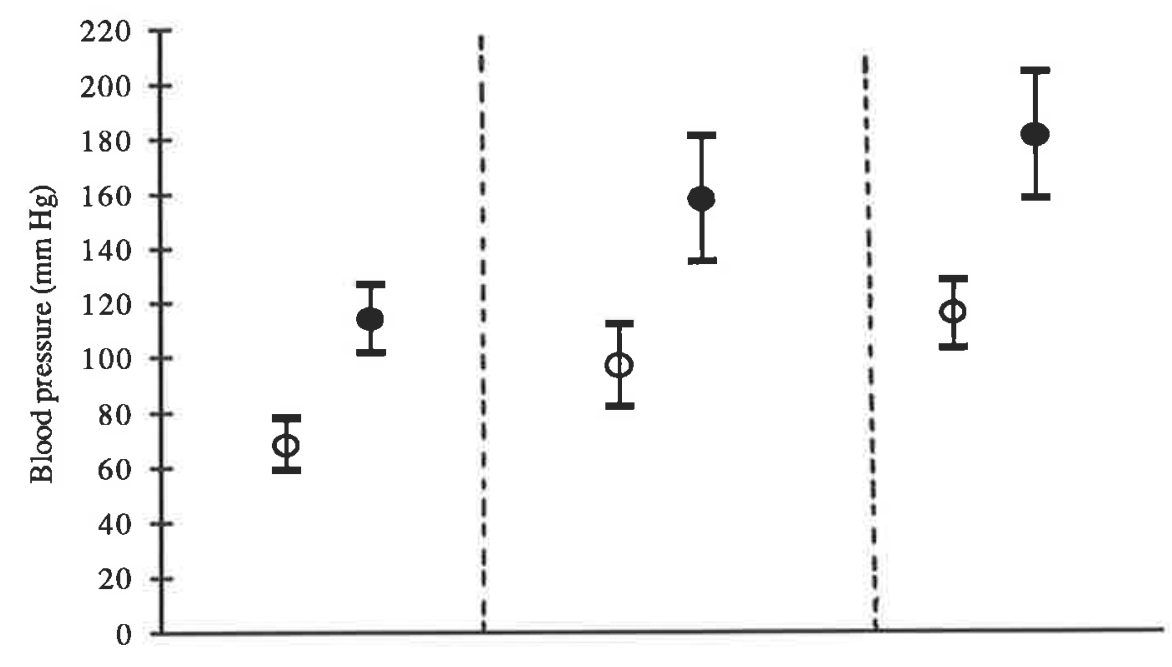

$\begin{array}{cccc} & \begin{array}{c}\text { Booking BP } \\ (\mathbf{n}=\mathbf{3 3 3})\end{array} & \begin{array}{c}\text { BP immediately before } \\ \text { seizure }(\mathbf{n = 2 0 1})\end{array} & \begin{array}{c}\text { Maximum BP } \\ (\mathbf{n}=\mathbf{3 7 7})\end{array} \\ \text { systolic mean }( \pm \text { SD) } & 114(12.8) & 158(22.6) & 181(23.0) \\ \text { diastolic meanO }( \pm \text { SD) } & 68(9.6) & 97(14.6) & 116(12.5)\end{array}$

Booking BP includes all 333 women who booked before 20 weeks gestation

BP immediately before seizure includes all 201 women who had BP measured within 1 hour of the seizure. Maximum BP recorded at any time during eclamptic pregnancy in 377 (cases missing data 6). 
4.2.5.2. Proteinuria.

Overall $97 \%$ (363/375 - missing data on 8$)$ of women had one or more + of proteinuria during their eclamptic illness. However $36 \%$ of these women (127/351 - missing data on 12) developed the proteinuria only after their first fit. Even amongst women hospitalised prior to eclampsia $30 \%(89 / 296)$ had no proteinuria recorded before the onset of seizures.

\subsubsection{Other signs of systemic involvement.}

Involvement of maternal hepatic and haematological systems in pre-eclampsia or eclampsia are diagnosed by looking for and finding disturbances in biochemical parameters.

Biochemical investigations were not routinely performed on women prior to the onset of seizures even in the 296 hospitalised women (See Table 4.7.). Amongst women who were investigated prior to the onset of seizures $52 \%(48 / 92)$ had elevated serum urate $(\geq 0.4$ $\mathrm{mmol} / \mathrm{l}), 23 \%(14 / 60)$ had raised liver transaminase levels ( $\geq 42 \mathrm{IU})$ and $13 \%(19 / 152)$ had thrombocytopaenia (platelet count $\leq 10 \times 10^{9} / 1$ ).

After the onset of eclampsia measurements of urea, creatinine and platelets were done on almost all women however, liver function tests were still not routinely performed (Table 4.7.).

After the onset of eclampsia 74\% (150/203) of women tested had elevated serum urate, $56 \%(134 / 240)$ had elevated transaminase levels and 34\% (126/374) had thrombocytopenia. In $46(12 \%)$ women the thrombocytopenia was severe enough to require a platelet transfusion. One-hundred and twelve women (29\%) required a blood transfusion during their hospital admission for eclampsia.

\subsubsection{Symptoms.}

Two-hundred and twenty-seven (59\%) women had one or more antecedent symptoms including headaches in $188(50 \%)$, visual disturbances in $72(19 \%)$ and epigastric pain in 71 $(19 \%)$. Fourteen women (4\%) had all three of the above symptoms before convulsing. 
TABLE 4.7. Investigations carried out before and after first eclamptic seizure in hospitalised women.

\begin{tabular}{lcccc}
\hline \multicolumn{1}{r}{ Investigation } & \multicolumn{2}{c}{$\begin{array}{c}\text { Before first seizure } \\
(\mathbf{n = 2 9 6 )}\end{array}$} & \multicolumn{2}{c}{$\begin{array}{c}\text { After first seizure } \\
(\mathbf{n}=\mathbf{3 8 1}) \ddagger\end{array}$} \\
& No. & \% & No. & \% \\
\hline Serum Urea & 133 & 45 & 369 & 97 \\
Serum Urate & $\dagger 92$ & 31 & $\S 188$ & 53 \\
Serum Creatinine & 114 & 39 & $\dagger 345$ & 91 \\
Serum Bilirubin & 63 & 21 & $\dagger 273$ & 72 \\
Serum AST/ALT & 60 & 20 & $\dagger 240$ & 63 \\
Platelet Count & 152 & 51 & 374 & 98 \\
APTT/INR & 105 & 35 & 334 & 88 \\
\hline
\end{tabular}

*Includes all women admitted to hospital before the onset of first seizure (missing data on 1). † missing data on $1 \ddagger$ missing data 2 . § information not collected on first 29 cases. AST - aspartate transaminase. ALT - alanine transaminase. APTT - activated partial thromboplastin time. INR - international normalised ratio.

\subsubsection{Antepartum, Intrapartum, Postpartum.}

One-hundred and forty-seven women (38\%) had antepartum eclampsia, 68 women (18\%) intrapartum and $168(44 \%)$ postpartum eclampsia. Twenty (12\%) of the postpartum cases occurred more than 48 hours after delivery, 3 (2\%) more than 7 days after delivery.

FIGURE 4.4. Number of cases of eclampsia by gestational age at first seizure.

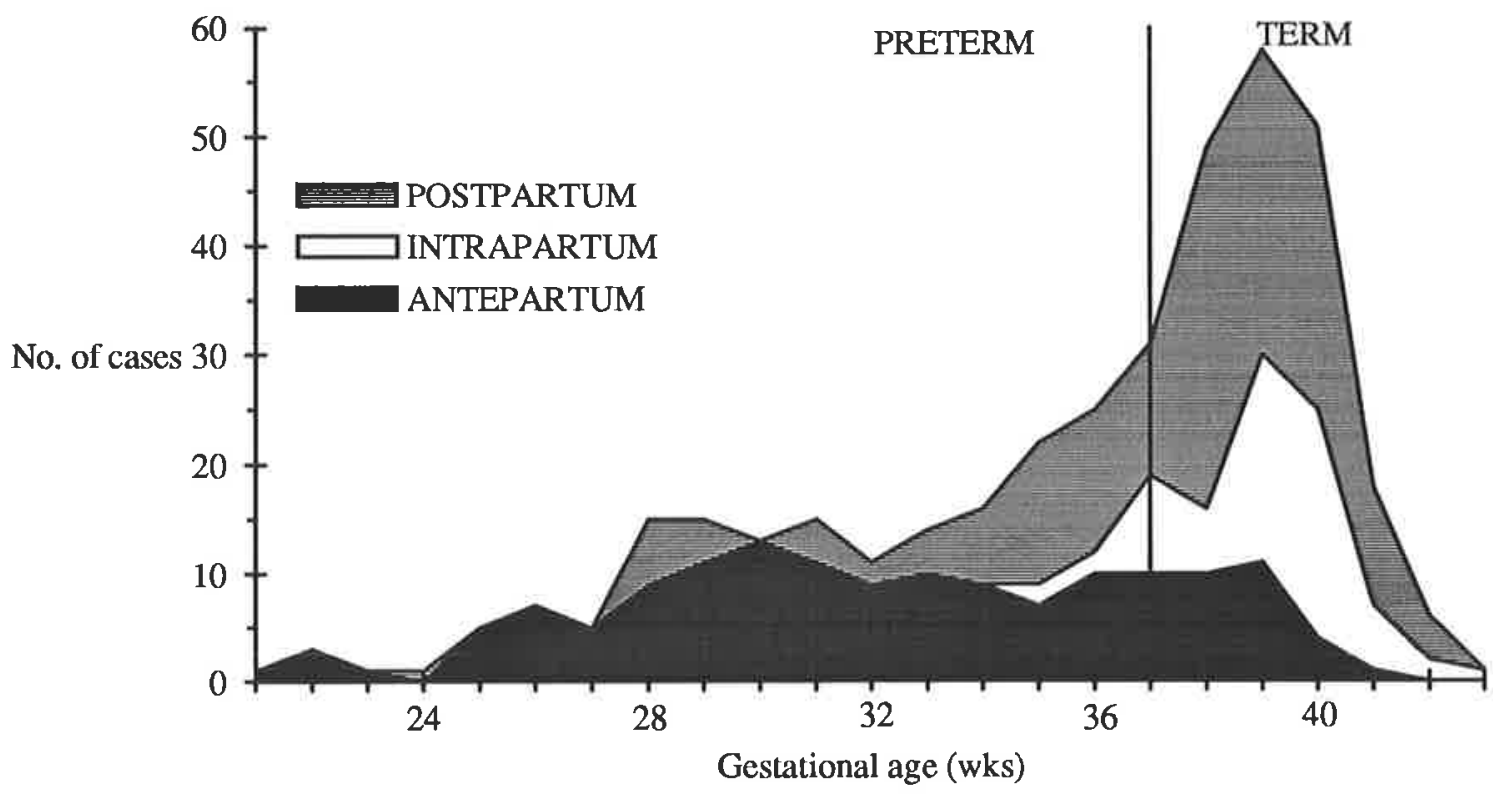

For post-partum cases gestational at first seizure equals gestational age at delivery. 


\subsubsection{Gestation of seizures.}

The mean gestational age at onset of seizures was 36 weeks although the range was from 21 weeks and 1 day to 43 weeks. Two hundred and fourteen $(56 \%)$ women had their first seizure at term (i.e. at or after 37 weeks of completed gestation). One-hundred and sixtynine women (44\%) had their first seizure preterm; 81 (21\%) occurring before 32 weeks completed gestation and $88(23 \%)$ between 32 and 37 weeks.

Figure 4.4. above shows the number of cases of eclampsia by gestational age at first seizure and divided into antepartum, intrapartum and postpartum seizures.

\subsubsection{Multiple seizures.}

One-hundred and fifty-eight (41\%) women had more than one seizure; 59 of these women had their first seizure outside hospital and 99 first fitted in hospital. Women who developed their first seizure outside of hospital were at a significantly greater risk of having multiple seizures than women whose first fit was in hospital (relative risk (95\% confidence interval) $2.10(1.70<\mathrm{RR}<2.60))$.

\subsubsection{Management of eclampsia.}

\subsubsection{Prophylactic medications.}

Prophylactic antihypertensives were used in 111 women altogether. They were used in $37 \%(109 / 296)$ of women in hospital prior to the onset of seizures and 50\% (92/183) of women with established hypertension and proteinuria. Thirty-two percent $(22 / 69)$ of women in hospital with maximum diastolic blood pressures before the onset of seizures $\geq$ $120 \mathrm{~mm} \mathrm{Hg}$ did not receive prophylactic antihypertensives.

Prophylactic anticonvulsants were used in $21 \%$ (61/296) of women admitted to hospital and $28 \%(51 / 183)$ of the women with established hypertension and proteinuria.

\subsubsection{Medications used to treat eclampsia.}

After the onset of seizures 373 (98\%) women were given some form of anticonvulsant and $299(78 \%)$ were given at least one antihypertensive medication. Only $6(2 \%)$ women had no medications at all following their eclamptic seizures.

Diazepam was the first medication used in $300(79 \%)$ cases. It was usually given as an immediate intravenous dose to cease seizure activity. The most common anticonvulsants used in the management of women with eclampsia were diazepam in 327 cases $(86 \%)$, 
phenytoin in 204 (53\%) cases, chlormethiazole in 71 (19\%) and magnesium sulphate in 11 (3\%) of cases. Barbiturates were used in 2 cases $(0.5 \%)$.

The most commonly used intravenous antihypertensives were hydralazine (204 - 53\%) and labetalol (86 - 23\%). Oral antihypertensives commonly used were labetalol (123 - 32\%) nifedipine (92 - 24\%) methyl dopa (24 - 6\%) and atenolol (19-5\%).

Other medications that were utilised less commonly included diuretics in 24 (6\%), dopamine in $17(4 \%)$, general anaesthesia to control seizures in $12(3 \%)$ and mannitol in $9(2 \%)$ women.

\subsubsection{Maternal Outcome.}

Two-hundred and six (54\%) women were delivered by caesarean section and 177 (46\%) had either spontaneous or instrumental vaginal deliveries. The caesarean section rate was much higher in women with antepartum seizures (92\%) and in women delivered pre-term $(79 \%)$.

\subsubsection{Maternal mortality.}

There were 7 maternal deaths, giving a maternal case fatality rate of $1.8 \%$ (95\% confidence interval $(0.7 \%-3.7 \%)$ ) Three of the women who died had antepartum eclampsia, three had intrapartum seizures and only one had postpartum eclampsia (Table 4.8.). In addition to the maternal deaths one woman was left in a persistent vegetative state following a massive cerebrovascular haemorrhage . 
TABLE 4.8. Maternal deaths associated with eclampsia in 1992

\begin{tabular}{|c|c|c|c|c|}
\hline $\begin{array}{l}\text { Age } \\
\text { (yrs) }\end{array}$ & Parity & $\begin{array}{l}\text { Type of } \\
\text { fit }\end{array}$ & $\begin{array}{l}\text { Gest } \\
\text { of fit }\end{array}$ & Outcome \\
\hline 27 & $\mathrm{G}_{2} \mathrm{P}_{n+1}$ & AN & 30 & Sepsis, RF, Resp F, CRA. Died day 10 \\
\hline 27 & $\mathrm{G}_{1} \mathrm{P}_{\mathrm{n}}$ & AN & $29+$ & $\begin{array}{l}\text { Status eclampticus on admission. Infant stillborn. ARDS DIC, RF, } \\
\text { Cerebral oedema. Died day } 32\end{array}$ \\
\hline 43 & $\mathrm{G}_{7} \mathrm{P}_{6}$ & AN & $40+$ & $\begin{array}{l}2 \text { seizures-stabilised \& discharged day } 8 \text { Day } 9 \text { re-admitted HT, PO, } \\
\text { Sepsis, ARDS, RF, DIC, ruptured liver. Died day } 21\end{array}$ \\
\hline 24 & $\mathrm{G}_{1} \mathrm{P}_{0}$ & $\mathbb{P}$ & $36+$ & $\begin{array}{l}\text { Aspiration, Mendlesons syndrome, CRA, Resp F., Legionella, Sepsis. } \\
\text { Died day } 28\end{array}$ \\
\hline 29 & $\mathrm{G}_{7} \mathrm{P}_{1}$ & IP \& PP & $37+$ & $\begin{array}{l}\text { Precipitous delivery at home. Multiple seizures HT, RF, DIC, PO, Liver } \\
\text { necrosis, CVA, Died day } 11\end{array}$ \\
\hline 34 & $\mathrm{G}_{4} \mathrm{P}_{3}$ & IP & $35+$ & 2 seizures DIC, RF, ARDS. Died day 26 \\
\hline 31 & $\mathrm{G}_{1} \mathrm{P}_{0}$ & PP & 38 & $\begin{array}{l}\text { Twin pregnancy. PO Multiple seizures CRA Cerebral hypoxia. Died day } \\
5\end{array}$ \\
\hline
\end{tabular}

HT-hypertension. RF-renal failure DIC-disseminated intravascular coagulopathy PO-pulmonary oedema CVA-cerebrovascular accident ARDS- adult respiratory distress syndrome Resp F-respiratory failure CRA cardio-respiratory arrest.

\subsubsection{Maternal morbidity.}

Hospital based morbidity.

One-hundred and forty-eight (39\%) women had at least one complication of delivery; the complications most commonly recorded were haemorrhage ( $\geq 500 \mathrm{ml}$ ) in $87(23 \%)$, pyrexia $\left(\geq 38^{\circ} \mathrm{C}\right)$ in $59(15 \%)$, retained products of conception in $15(4 \%)$ and wound infection in 11 $(3 \%)$.

The length of hospital stay after eclampsia varied from 2 to more than 40 days, 46 (12\%) women stayed more than two weeks and $19(5 \%)$ for more than three weeks. One-hundred and seventy-two $(45 \%)$ of the women were admitted to an intensive care unit and 135 women (35\%) had at least one major complication (Table 4.9.).

\section{General practitioner reported morbidity.}

One-hundred and ninety-four $(63 \%)$ of the 306 women whose general practitioners responded had some documented post eclamptic morbidity. Hypertension was the most frequent problem but anxieties about personal health, future pregnancies, headaches and depression were also common (see Table 4.10.). 
TABLE 4.9. Major maternal complications recorded in hospital case-notes.

\begin{tabular}{lcc}
\hline \multicolumn{1}{c}{ Major maternal complications } & $\begin{array}{c}\text { No. of cases } \\
\mathbf{n = 3 8 3}(*)\end{array}$ & $\begin{array}{c}\text { \% of } \\
\text { cases }\end{array}$ \\
\hline Death & 7 & 2 \\
Persistent vegetative state & 1 & $<1$ \\
Cardiac arrest & $6(4)$ & 2 \\
Cerebro-vascular accident & $7(1)$ & 2 \\
Adult respiratory distress syndrome & $7(3)$ & 2 \\
HELLP syndrome $\dagger$ & $27(2)$ & 7 \\
DIC $¥$ (other than HELLP syndrome) & $33(3)$ & 9 \\
Pulmonary oedema & 18 & 5 \\
Aspiration-Mendlesons syndrome & $4(1)$ & 1 \\
Renal failure & $24(4)$ & 6 \\
Pulmonary embolus & 5 & 1 \\
Septicaemia & $2(1)$ & $<1$ \\
Cortical blindness (transient) & 2 & $<1$ \\
Required ventilation & $87(6)$ & 23 \\
\hline
\end{tabular}

* The figures in brackets are the complications suffered in the 7 fatal cases of eclampsia. † HELLP syndrome = Haemolysis, Elevated Liver enzymes and Low Platelets syndrome. $¥$ DIC $=$ Disseminated Intravascular Coagulopathy

TABLE 4.10. Post eclamptic maternal morbidity recorded by general practitioners.

\begin{tabular}{lcc}
\hline $\begin{array}{l}\text { Maternal morbidity recorded } \\
\text { by GP }\end{array}$ & $\begin{array}{c}\text { No. of } \\
\text { cases } \\
(\mathbf{n = 3 0 6})\end{array}$ & $\begin{array}{c}\text { \% of } \\
\text { cases }\end{array}$ \\
\hline Hypertension & 69 & 23 \\
Anxiety about own health & 44 & 14 \\
Anxiety about future pregnancies & 43 & 14 \\
Headaches & 37 & 12 \\
Anxiety about child & 30 & 10 \\
Depression & 29 & 10 \\
Vaginal bleeding & 23 & 8 \\
Perineal/wound pain & 22 & 7 \\
Urinary tract infection & 16 & 5 \\
Neurological deficit & 12 & 4 \\
Memory loss & 11 & 4 \\
Other & 25 & 8 \\
\hline
\end{tabular}

$326 / 383$ general practitioner questionnaires returned but in 20 cases information on morbidity was missing. 


\subsubsection{Fetal Outcome.}

There were 411 fetuses; 356 singletons, 26 twins and 1 set of triplets. Two hundred of the infants were male and 211 female giving a male : female sex ratio of 0.95 .

\subsubsection{Fetal mortality.}

Thirty fetal and infant deaths were reported giving a total study perinatal mortality of $7.3 \%$. Five of these were intrauterine deaths in women who were delivered between 20 and 24 weeks gestation because of worsening maternal condition. There were nine stillbirths in fetuses over 24 weeks gestation (stillbirth rate of 22.2/1000 95\% confidence interval (10.2 41.7 )) and thirteen infants died in the neonatal period (neonatal mortality rate of $32.0 / 1000$ (17.2 - 54.1)). In addition three deaths were reported from complications of prematurity in infants aged more than 4 weeks.

All 30 fetal/infant deaths were singletons and all occurred in women with onset of eclampsia before 38 completed weeks of pregnancy, $24(75 \%)$ in women with eclampsia before 32 completed weeks of pregnancy. Twenty-four $(75 \%)$ of the deaths occurred in infants/fetuses weighing less than 1500 grams and $18(60 \%)$ of them weighed less than 1000 grams. Twenty-four $(75 \%)$ fetal/infant deaths occurred in women whose first fit had been antepartum, $2(7 \%)$ in women with intrapartum seizures and $4(13 \%)$ in women with postpartum seizures.

Follow-up on infants was difficult to obtain because there was no access to the infants casenotes. Wherever possible information was obtained from general practitioners and special care baby units. There were 7 cases where the infant was retained on the special care baby unit after the mothers discharge and where it could not be confirmed that the infant had been discharged alive. In 6 of these cases the infants birth weight was between $1000-1500$ grams. With this incomplete follow-up in mind the neonatal case fatality rate must be treated with caution. The true figure may be higher but is unlikely to be any lower.

\subsubsection{Fetal morbidity.}

All but one of the fetuses were weighed at delivery: $35(9 \%)$ weighed 1000 grams or less and $44(11 \%)$ weighed between 1001 and 1500 grams. Of the 356 singletons $100(28 \%)$ were small for gestational age. Two-hundred and twenty (56\%) of the 397 live born infants were admitted to special care baby units.

Of the 381 surviving infants some information on morbidity was available from either the special care baby unit or from the general practitioner in $346(90 \%)$ cases. Sixty-nine 
(20\%) of these infants had at least some morbidity reported. Specific types of fetal morbidity are shown in Table 4.11 .

TABLE 4.11. Morbidity in surviving infants recorded by special care baby units or the general practitioners

\begin{tabular}{lcc}
\hline \multicolumn{1}{c}{ Infant morbidity } & $\begin{array}{c}\text { No. of cases } \\
\left(\mathbf{n}=\mathbf{3 4 6}^{*}\right)\end{array}$ & $\begin{array}{c}\text { \% of } \\
\text { cases }\end{array}$ \\
\hline Respiratory problems & 40 & 12 \\
Hydrocephalus & 3 & 1 \\
Neurological deficit & 6 & 2 \\
Congenital heart disease & 8 & 2 \\
Other congenital abnormalities & 8 & 2 \\
Sepsis & 3 & 1 \\
Jaundice & 10 & 3 \\
Hypoglycaemia & 6 & 2 \\
Others & 23 & 7 \\
\hline
\end{tabular}

* 381 surviving infants, missing information on 35

\subsection{DISCUSSION.}

\subsubsection{A true picture of eclampsia?}

Much of the time planning and implementing the study was spent in trying to ensure that every case of eclampsia was notified and that data collection was maximised. Gratifyingly it appears these efforts paid off: there was a very high response $(95 \%)$ to request for notifications, a tendency to over notify possible cases and, in comparison with existing systems, the ascertainment of cases was complete. There were also very high rates of casenote review and return of completed general practitioner questionnaires.

The high rate of data collection on individual cases was not easy to achieve; although there was no active resistance to supplying information, protracted delays and passive obstacles were frequently encountered and were only overcome by persistence. The value of the persistence was shown by the fact that 2 of the 7 maternal deaths were amongst the last 5 sets of case-notes received, thus reinforcing the need for complete data collection in studies of this kind.

The completeness of ascertainment is reassuring for this study but tends to cast doubt on the validity of the established systems of maternity data collection. It is particularly 
concerning with regard to the Confidential Enquiries into Maternal Deaths (CEMD) which has a reputation for complete data collection. In fact the CEMD notification system is notoriously slow so they may well have eventually received notifications for all the of deaths recorded by this study; never-the-less, it is concerning that several 1992 deaths were still outstanding at the end of 1994.

In summary, the total ascertainment together with the completeness of the data collection mean that the incidence and maternal case-fatality rates for eclampsia reported in this study are likely to be accurate. Further-more this study can claim to represent a comprehensive view of eclampsia in the United Kingdom during 1992.

\subsubsection{Descriptive results.}

The incidence of $4.9 / 10,000$ found in this study is similar to that reported for the USA $(4.3 / 10,000)$ in $1983-86$ (Saftlas et al. 1990) but higher than that in Sweden $(2.7 / 10,000)$ in 1980 (Moller \& Lindmark, 1986). The inclusion criteria are similar in this and the USA study but the Swedish study employed broader criteria. Thus, the difference in incidence between Sweden and the other two countries may be greater.

There were substantial differences in the incidence rates of eclampsia in different regions of the English National Health Service. The rate in the Northern region was more than twice that of the Oxford region. However, it should be remembered that the absolute numbers of cases are small and may vary considerably from year to year. The reasons for the difference in incidence are not known although they may be explained by demographic and socioeconomic differences between the regions.

There is no doubt that there has been a marked reduction in the incidence of eclampsia in the United Kingdom since 1922 (when the rate was $(80 / 10,000)$ (Eden, 1922)) but the evidence for any significant decrease in the last 30 years is less compelling. The regional incidences suggest there has been little if any improvement over the last two decades. There were 5.3 cases per 10,000 deliveries amongst residents of South Glamorgan in 196574 (Wightman et al. 1978), compared with 4.0/10,000 maternities for all of Wales in this study. In the Grampian region of Scotland in 1978-83 the rate was $8 / 10,000$ (Hall \& Campbell, 1992) compared with our data for the whole of Scotland of 6.7/10,000. Criteria for eclampsia were not explicitly stated in the above studies, but from the limited information provided it is clear that we would have excluded some of their cases.

In New Zealand, eclampsia was a notifiable disease for many years and its incidence declined from $32 / 10,000$ to $8 / 10,000$ between 1928-33 and 1956-58 (Corkill, 1961); a 
reduction Corkhill attributed to the advent of routine screening for early signs of preeclampsia (Corkill, 1961). The reduction in incidence in the UK between the 1920's and 1970 's also occurred as antenatal care became universally available. This, coupled with the fact that countries without effective antenatal screening programmes still have much higher incidence rates (Moodley et al. 1983; Porapakkham, 1979; Moore \& Munoz, 1985) provides circumstantial evidence to support Corkhill's hypothesis.

The majority of cases of eclampsia in this study occurred in spite of a standard frequency of antenatal assessments (70\%) and even after hospitalisation (77\%). Furthermore, seizures were frequently $(38 \%)$ unheralded by hypertension and proteinuria, even in hospitalised women, so it is not surprising that they could not be prevented. Unheralded eclampsia has been noted before, but in proportionally fewer cases (about 1 in 6) (Sibai et al. 1986a; Campbell \& Templeton, 1980).

Corkhill's hypothesis and this studies findings are compatible. Screening by blood pressure measurement and urinalysis may prevent most eclampsia preceded by hypertension and proteinuria (evidenced by the reduction in incidence in the UK between 1922 and 1970). However, as eclampsia with a classical presentation is prevented, atypical presentations become a proportionately greater problem (hence the relatively stable incidence since the 1970's and the clinical findings in this study). The implication of this is that to achieve further significant reductions in the incidence of eclampsia new screening and diagnostic procedures which do not rely on hypertension and proteinuria need to be developed.

In this study as many as one third of women had only mild hypertension before the onset of seizures and in these cases there were often delays in making a diagnosis, apparently, because of the clinicians belief that eclampsia only occurs in the context of severe symptomatic proteinuric pre-eclampsia (see Figure 1.1.). The clinical picture of eclampsia described here is more consistent with the theory that seizures are one of a range of signs and symptoms of pre-eclampsia which can appear in any order and are caused by widespread endothelial cell damage secondary to an ischaemic placenta (see Figure 1.2.) (Roberts \& Redman, 1993). Greater acceptance of this theory may lead to quicker diagnosis and initiation of management in cases of eclampsia.

Hyperuricaemia, thrombocytopenia, and abnormal plasma concentrations of liver enzymes are well recognised signs of pre-eclampsia and give clinicians information about the extent of maternal systemic involvement. Monitoring these indices can give early warning of impending de-compensations such as HELLP (haemolysis, elevated liver enzymes and low platelets) syndrome (Weinstein, 1982) or renal failure. Recent reports of the Confidential 
Enquiries into Maternal Mortality have advocated monitoring biochemical indices (DHSS et al. 1991) so it was disappointing to find that tests were performed infrequently in women until after the onset of seizures and were not uniformly done even then.

In 1992 there was no generally accepted "best" management for pre-eclampsia or eclampsia so it is impossible to compare the treatment given with any standard. It is however possible to compare how consultant obstetricians said they would manage patients in a 1991 questionnaire study (Hutton et al. 1992) with the management actually used in 1992. Ninety-eight percent of respondents to the survey stated they would use prophylactic antihypertensives in women with diastolic blood pressure $\geq 120 \mathrm{~mm} \mathrm{Hg}$ (Hutton et al. 1992), however, in this study only $68 \%$ of women with such high readings prior to the onset of seizures received antihypertensives prophylactically. Similarly, $85 \%$ of obstetricians claimed to use prophylactic anticonvulsants in women with pre-eclampsia (Hutton et al. 1992) but in this series only $28 \%$ of women in hospital with established proteinuria and hypertension received such medication. There seems to be a considerable difference between what consultants intend to do and what is actually done. Alternatively, prophylactic medications may be used frequently and effectively in the majority of cases of pre-eclampsia and the eclamptic population in this study is biased towards women who did not receive prophylaxis or in whom it was ineffective.

This study has measured the maternal case-fatality rate of eclampsia in the United Kingdom for the first time. The rate of just under 1 in 50 (1.8\%) shows that eclampsia is still a marker of an extremely dangerous disorder. Further evidence of its danger is the high rate of major maternal complications (39\%). However, it is not the convulsions themselves that are hazardous (idiopathic epilepsy has a much lower maternal case fatality rate) but the severity of the underlying disturbances. Therefore the focus of secondary prevention in preeclampsia should not be simply to prevent convulsions in a desperate situation, but to prevent the dangerous situation itself.

This is the first study which reports on minor morbidity in the months following an eclamptic seizures. General practitioners reported residual morbidity in $63 \%$ of women following eclampsia. This may represent a biased sample as GP's whose patients have experienced some morbidity may be more likely to answer and return the questionnaire. Never-the-less recognition of the minor morbidity needs to be accompanied by attempts to reduce it. A first step would be to ensure that all women with eclampsia are given followup appointments at the specialist unit so that they can express concerns and seek information about future pregnancies. 
The stillbirth rate and neonatal mortality rates in this study are high compared to national rates but are lower than those previously reported in series of eclamptic women (Sibai, 1990a; Wightman et al. 1978; Gedekoh et al. 1981). The reduction in perinatal mortality in this compared to earlier studies is probably a reflection of continuing improvements in the care of premature infants. 


\section{CHAPTER 5.}

\section{SUBGROUP ANALYSIS AND LOGISTIC REGRESSION RESULTS AND DISCUSSION 2}


Chapter 4 described the data collected on the whole population of women with eclampsia and in this chapter subgroups are compared to assess if there are significant differences between the groups. The prior hypotheses (listed in section 1.8.3.) which were derived from consideration of the available literature are individually tested in the following sections.

\subsection{ANTENATAL CARE - WHAT IS ITS ROLE?}

5.1.1. Prior hypothesis: A significant proportion of eclampsia occurs in women who have had a long interval between formal screening checks for the signs of preeclampsia.

\subsubsection{Results.}

Three percent of women (13/382 missing information in 1) had no antenatal care before the onset of eclampsia and a further $29 \%$ (85/291 missing information on 79 ) received care that did not conform to the traditional pattern of antenatal care in the United Kingdom. However $77 \%(296 / 383)$ of women had their first seizure in hospital where frequent blood pressure and urine checks were made.

Overall, only 13\% (47/371 missing information on 12) of all women had an interval between last check on blood pressure and first seizure of more than 7 days and 6\% (23/371) had an interval of more than 14 days. Even in the 98 women with less than traditional antenatal care the proportion of women with a screening interval between last check on blood pressure and first seizure of more than 14 days was only $12 \%$ (11/89 missing information on 9).

Underlying this prior hypothesis is the thought that frequent screening tests may allow early intervention in pre-eclampsia and help to prevent eclampsia. It seems unreasonable to expect that antenatal care (no matter how frequent) could prevent seizures that occur in labour or after delivery therefore the analysis was repeated including only women with antenatal eclampsia. One hundred and forty-seven women had antepartum seizures: $34 \%$ of these women (46/136 missing data on 11) had an interval between last blood pressure check and seizure of greater than 7 days and 17\% (23/136) had an interval greater than 14 days. The interval between last check on proteinuria and seizure was more than 7 days in $43 \%$ (57/132 missing information in 15) and more than 14 days in 23\% (30/132) of women.

In summary, only $6 \%$ of all women in this series had an interval of greater than 14 days between the last check on blood pressure and the first seizure. However if only women 
with antenatal seizures are considered then the proportion with a long screening interval rises to one sixth (17\%).

5.1.2. Further questions: Do women who have long antenatal screening intervals prior to the onset of eclampsia develop more complications than those women who receive frequent screening for pre-eclampsia?

This analysis was performed on the subgroup of women with antenatal eclampsia as it is in this group that antenatal care is likely to make a difference. Women with a long screening interval were significantly more likely to have their first seizure outside of the hospital (relative risk (95\% confidence interval) of $2.35(1.90-2.92)$ ) and to have multiple seizures (relative risk $1.93(1.49-3.50)$ ) than women who were screened more frequently. Women with long screening intervals also had higher maximum blood pressures, more preterm seizures, more major maternal complications, more admissions to intensive care units, greater maternal mortality, and poorer fetal/infant outcomes as shown in Table 5.1. however none of these differences reached statistical significance.

TABLE 5.1. Differences in outcome between women with antenatal eclampsia who had a long interval (>14 days) between tests on blood pressure and those women with antenatal eclampsia who had more frequent ( $\leq 14$ days) screening checks.

\begin{tabular}{|c|c|c|c|c|c|}
\hline \multirow[t]{2}{*}{ Factor of interest } & \multicolumn{2}{|c|}{$\begin{array}{c}\text { Long screening } \\
\text { interval (>14 days) } \\
n=23\end{array}$} & \multicolumn{2}{|c|}{$\begin{array}{c}\text { Screening } \\
\text { interval } \leq 14 \text { days } \\
n=113\end{array}$} & \multirow{2}{*}{$\begin{array}{c}\text { Statistical test } \\
\text { Chi square (Yates) } \\
\text { Relative risk ( } 95 \% \mathrm{CI})\end{array}$} \\
\hline & No. & $\% / S D$ & No. & $\% / \mathrm{SD}$ & \\
\hline Maximum diastolic BP (mean \& SD) & 123 & 12.1 & 117 & 15.9 & $* \mathrm{p}=0.112$ \\
\hline Preterm seizures & 20 & 87 & 82 & 73 & $1.41 \mathrm{p}=0.235$ \\
\hline First fit outside of hospital & 23 & 100 & 48 & 43 & $\begin{array}{c}23.1 \mathrm{p}<0.001 \\
2.35(1.90-2.92)\end{array}$ \\
\hline Multiple seizures & 20 & 87 & 50 & 45 & $\begin{array}{c}11.8 p<0.001 \\
1.93(1.49-3.50)\end{array}$ \\
\hline Admission to Intensive care unit & 16 & 70 & 63 & 56 & $0.98 \mathrm{p}=0.321$ \\
\hline Major maternal compllcation & 14 & 61 & 56 & 50 & $0.58 \mathrm{p}=0.447$ \\
\hline Maternal mortality & 2 & 9 & 1 & 1 & $\dagger p=0.074$ \\
\hline GP reported maternal morbidity & 11 & 65 & 68 & 70 & $0.03 p=0.873$ \\
\hline $\begin{array}{l}\text { Small for gestational age fetus } \\
\text { (amongst singletons) }\end{array}$ & 12 & 52 & 46 & 43 & $0.37 p=0.543$ \\
\hline Fetal/infant mortality & 6 & 26 & 17 & 15 & $\dagger p=0.153$ \\
\hline $\begin{array}{l}\text { Infant morbidity (amongst surviving } \\
\text { infants) }\end{array}$ & 9 & 56 & 29 & 32 & $2.55 \mathrm{p}=0.110$ \\
\hline
\end{tabular}

147 women had antepartum eclampsia but information on screening interval was missing in 11 therefor this table includes 136 women. * - Kruskal-Wallis $\mathrm{H}$ test. $\dagger$ - Fisher exact test. 


\subsection{UNHERALDED ECLAMPSIA.}

5.2.1. Prior hypothesis: Eclampsia occurs in some women without prodromal symptoms and signs.

In order to test the hypothesis it was necessary to select a group of women who had been checked recently for pre-eclampsia and did not have it, rather than just women who were not recorded as having pre-eclampsia (which may have been because it had not been looked for). Eclampsia was said to have occurred without prodromal signs (i.e. to have been unheralded) if the woman had a urine screen and blood pressure measurement in the 24 hours preceding the onset of seizures and did not have established proteinuria and hypertension.

\subsubsection{Results.}

Overall there were $184(48 \%)$ women who did not have confirmed proteinuria and hypertension before their first seizure but many of these women had last been seen several days or even weeks prior to the onset of seizures.

Two-hundred and sixty-two women had urine screened and blood pressure measured in the 24 hours prior to the onset of eclampsia. Eighteen $(7 \%)$ of these women had neither hypertension nor proteinuria, $48(18 \%)$ had hypertension alone, $20(8 \%)$ had proteinuria alone and $176(67 \%)$ had proteinuria and hypertension. Therefore $33 \%$ (86) of all women screened in the 24 hours prior to the onset of seizures did not have the classical duo of preeclampsia signs and $7 \%$ had neither sign.

In the same group of 262 recently screened women $110(41 \%)$ had no symptoms of headache, epigastric pain or visual disturbance before developing eclampsia. Fifteen $(6 \%)$ of these 262 women developed eclampsia despite the fact that 24 hours before the first seizure they had no signs or symptoms of pre-eclampsia.

In summary eclamptic seizures do occur in appreciable numbers of women who do not have classical prodromal signs or symptoms.

5.2.2. Further questions: Do women who develop eclampsia without the classical prodromal illness have a fewer complications?

The 86 women with unheralded eclampsia were compared with 176 women who did have established proteinuric hypertension prior to eclampsia to see if there were significant differences in the two groups with regard to maternal and fetal outcomes (Table 5.2.). 


\subsubsection{Results}

Unheralded eclampsia was more likely to occur at term with a relative risk (95\% confidence interval) of 1.52 (1.29 - 1.79). Women with unheralded eclampsia had fewer major maternal complications (relative risk of $0.50(0.30-0.83)$ ), fewer admissions to intensive care units (relative risk $0.65(0.45-0.94)$ ) and fewer small for gestational age infants (relative risk $0.41(0.21-0.78)$ ). There were no statistical differences between the two groups in either maternal mortality or total fetal/infant mortality.

Later (section 5.6.) it is shown that eclampsia which occurs at term has fewer maternal and fetal complications than preterm eclampsia. The data on heralded and unheralded eclampsia were therefore stratified to take into account the gestation at which they occurred and were re-analysed. Women with unheralded eclampsia had fewer major maternal complications than women with eclampsia preceded by proteinuric hypertension (relative risk of 0.55 $(0.32-0.95))$ and this association was independent of whether the seizure occurred at term or preterm. No other associations remained significant once the relationship between term and unheralded seizures was accounted for (Table 5.2.).

In summary women with eclampsia that is unheralded by proteinuric hypertension have poorer maternal outcomes and fewer small for gestational age fetuses. However most of the significant differences are explained by the higher proportion of term seizures in this group. 
TABLE 5.2. Differences between women whose first fit was not preceded by proteinuric hypertension and those who did have established proteinuria and hypertension before their first fit.

\begin{tabular}{|c|c|c|c|c|c|c|}
\hline \multirow[t]{2}{*}{ Factor of interest } & \multicolumn{2}{|c|}{$\begin{array}{c}\text { Unheralded } \\
\text { eclampsia } \\
n=86\end{array}$} & \multicolumn{2}{|c|}{$\begin{array}{c}\text { Heralded } \\
\text { eclampsia } \\
n=176\end{array}$} & \multirow{2}{*}{$\begin{array}{c}\text { Statistical test } \\
\text { Chi square (Yates) } \\
\text { Relative risk (95\% CI) }\end{array}$} & \multirow{2}{*}{$\begin{array}{l}\text { Results of stratified } \\
\text { analysis } \\
\text { M-H Sum. Chi Square } \\
\text { Sum. relative risk ( } 95 \% \\
\text { CI) }\end{array}$} \\
\hline & No. & $\%$ & No. & $\%$ & & \\
\hline Term & 72 & 84 & 97 & 55 & $\begin{array}{c}19.42 p<0.001) \\
1.52(1.29-1.79)\end{array}$ & NA \\
\hline Admission to intensive care unit & 25 & 29 & 79 & 45 & $\begin{array}{c}5.39 \mathrm{p}=0.02 \\
0.65(0.45-0.94)\end{array}$ & $\begin{array}{c}3.57 p=0.059 \\
0.68(0.46-1.01)\end{array}$ \\
\hline Major maternal complication & 15 & 17 & 61 & 35 & $\begin{array}{c}7.50 p=0.006 \\
0.50(0.30-0.83)\end{array}$ & $\begin{array}{c}4.68 p=0.031 \\
0.55(0.32-0.95)\end{array}$ \\
\hline Maternal mortality & 0 & 0 & 4 & 2 & ${ }^{*} \mathrm{p}=0.201$ & NA \\
\hline GP reported maternal morbidity & 33 & 50 & 94 & 65 & $3.15 \mathrm{p}=0.076$ & NA \\
\hline $\begin{array}{l}\text { Small for gestational age fetus } \\
\text { (amongst } 356 \text { singletons) }\end{array}$ & 9 & 12 & 47 & 29 & $\begin{array}{c}7.68 \mathrm{p}=0.006 \\
0.41(0.21-0.78)\end{array}$ & $\begin{array}{c}1.05 p=0.307 \\
0.68(0.36-1.28)\end{array}$ \\
\hline Fetal/infant mortality & 4 & 5 & 13 & 7 & $0.38 \mathrm{p}=0.5359$ & NA \\
\hline $\begin{array}{l}\text { Infant morbidity (amongst } \\
\text { surviving infants) }\end{array}$ & 10 & 13 & 29 & 18 & $0.46 \mathrm{p}=0.498$ & NA \\
\hline
\end{tabular}

This table includes all 262 women who had both blood pressure and urine measured in the 24 hours prior to the onset of eclampsia. * - Fisher exact test used. NA Not applicable. Relative risk (95\% CI) is the relative risk (95\% confidence interval) of the factor for woman with unheralded eclampsia. Stratified analysis - the tables were stratified for the gestation at fit (i.e. preterm or term). M-H Sum. Chi square = Mantel- Haenszel Summary Chi square. Sum relative risk = the summary relative risk (95\% confidence interval) of the factor for women with unheralded eclampsia. 


\subsection{ECLAMPSIA AT THE EXTREMES OF MATERNAL AGE.}

5.3.1. Prior hypothesis: There is greater morbidity from eclampsia amongst older women.

\subsubsection{Results.}

Thirty-three $(9 \%)$ women were aged 35 or more when they developed eclampsia. This group of older women were more likely to have a significant past medical history (i.e. of hypertension, diabetes, auto-immune disorder or epilepsy) than the younger women (relative risk (95\% confidence interval) of $1.85(1.12-3.05)$ ) and were significantly less likely to be primigravidae (relative risk of $0.43(0.25-0.72)$. There were however no significant differences between the two groups in any of the maternal or fetal outcome measures as shown in Table 5.3.

Thus in this study the risk of maternal or fetal morbidity or mortality from eclampsia for women over the age of 35 was not significantly greater than for younger women. 
TABLE 5.3. Differences between women greater than or equal to 35 years of age versus all other women.

\begin{tabular}{|c|c|c|c|c|c|}
\hline \multirow[t]{2}{*}{ Factor of interest } & \multicolumn{2}{|c|}{$\begin{array}{c}\text { Women } \geq 35 \text { yrs } \\
n=33\end{array}$} & \multicolumn{2}{|c|}{$\begin{array}{c}\text { Women }<35 \text { yrs } \\
n=350\end{array}$} & \multirow{2}{*}{$\begin{array}{c}\text { Statistical test } \\
\text { Chi square (Yates) } \\
\text { Relative risk }(95 \% \mathrm{CI})\end{array}$} \\
\hline & No. & $\% / \mathrm{SD}$ & No. & $\% / \mathrm{SD}$ & \\
\hline *Past medical history & 12 & 36 & 68 & 20 & $\begin{array}{c}4.10 p=0.043 \\
1.85(1.12-3.05)\end{array}$ \\
\hline Primiparous & 10 & 30 & 276 & 79 & $\begin{array}{c}35.58 \mathrm{p}<0.001 \\
0.43(0.25-0.72)\end{array}$ \\
\hline Less than traditional antenatal care & 8 & 29 & 89 & 32 & $0.04 \mathrm{p}=0.844$ \\
\hline Prodromal symptoms & 23 & 72 & 204 & 59 & $1.49 \mathrm{p}=0.222$ \\
\hline Prodromal signs (seen within 7 days) & 23 & 85 & 266 & 89 & ${ }^{*} \mathrm{p}=0.349$ \\
\hline $\begin{array}{l}\text { Diastolic BP immediately before } \\
\text { seizure (mean \& SD) }\end{array}$ & 96 & 16.1 & 97 & 14.4 & $\dagger 0.076 p=0.783$ \\
\hline Maximum diastolic BP (mean \& SD) & 116 & 12.1 & 116 & 12.6 & $\uparrow 0.038 p=0.844$ \\
\hline First fit outside of hospital & 8 & 24 & 78 & 22 & $0.15 \mathrm{p}=0.698$ \\
\hline Delivery by caesarean section & 17 & 52 & 189 & 54 & $0.01 \mathrm{p}=0.927$ \\
\hline Antepartum & 11 & 33 & 136 & 39 & $0.19 \mathrm{p}=0.662$ \\
\hline Preterm seizures & 15 & 45 & 154 & 44 & $0.00 \mathrm{p}=0.982)$ \\
\hline Multiple seizures & 13 & 39 & 145 & 42 & $0.00 \mathrm{p}=0.945$ \\
\hline Major maternal complication & 12 & 36 & 126 & 35 & $0.00 \mathrm{p}=0.951$ \\
\hline Admission to intensive care unit & 13 & 39 & 159 & 45 & $0.23 \mathrm{p}=0.629$ \\
\hline Maternal mortality & 1 & 3 & 6 & 2 & $\ddagger p=0.471$ \\
\hline GP reported maternal morbidity & 16 & 57 & 178 & 64 & $0.24 \mathrm{p}=0.624$ \\
\hline $\begin{array}{l}\text { Small for gestational age fetus } \\
\text { (amongst } \mathbf{3 5 6} \text { singletons) }\end{array}$ & 11 & 36 & 89 & 28 & $0.51 \mathrm{p}=0.473$ \\
\hline Fetal/infant mortality & 4 & 12 & 26 & 7 & $\ddagger \mathrm{p}=0.228$ \\
\hline $\begin{array}{l}\text { Infant morbidity (amongst surviving } \\
\text { infants) }\end{array}$ & 8 & 28 & 21 & 19 & $0.65 \mathrm{p}=0.420$ \\
\hline
\end{tabular}

This table includes all 383 women with eclampsia. *Past medical history includes a past history of diabetes, epilepsy, renal disease or hypertension. $\dagger$ - Kruskal-Wallis $H$ test. $\ddagger$ - Fisher exact test used. 


\subsection{ECLAMPSIA IN MULTIPAROUS WOMEN.}

5.4.1. Prior hypothesis: A significant proportion of eclampsia occurs in multiparous women and these women have a greater morbidity from eclampsia.

\subsubsection{Results.}

The parity of women with eclampsia was known in all but one of the subjects of the study. Twenty-five percent (96) of the women were multiparous but only $1 \%$ (4) were grand multiparae (i.e. 5 or more previous viable pregnancies).

The mean age of multiparous women was 30 years (standard deviation 5.5) with a range from 19 years to 43 . The mean age of multiparous women was significantly higher than primiparae.

2

Twenty-six (27\%) of the multiparous women had a history of pre-eclampsia and $1(1 \%)$ had a history of eclampsia. There were no significant differences between multiparae and primiparae in terms of past medical history (i.e. a previous history of diabetes, epilepsy, hypertension or auto-immune disorder), the proportion with less than traditional antenatal care, the type of convulsion or the gestation at which first convulsion occurred. Nor did they differ in diastolic blood pressures, the proportion who fitted outside of hospital or in terms of the number with pre-fit hypertension or proteinuria. Multiparous women were significantly more likely to report the presence of prodromal signs of headache, epigastric pain or visual disturbances (Table 5.4.).

There were no significant differences between primiparous and multiparous women in terms of maternal outcome measures. Specifically no significant differences were found in the proportion with multiple seizures, major maternal complications, the proportion requiring admission to intensive care units or in maternal mortality or general practitioner reported morbidity rates. Similarly the outcome measures for infants were not significantly different in multiparous compared with primiparous women (Table 5.4.).

In summary eclampsia does occur in multiparous women. There is no evidence from this study that multiparous women have any greater maternal or fetal morbidity or mortality then primiparous women. 
TABLE 5.4. Differences between multiparous and primiparous women.

\begin{tabular}{|c|c|c|c|c|c|}
\hline \multirow[t]{2}{*}{ Factor of interest } & \multicolumn{2}{|c|}{$\begin{array}{c}\text { Multiparae } \\
\mathrm{n}=96\end{array}$} & \multicolumn{2}{|c|}{$\begin{array}{c}\text { Primiparae } \\
\mathrm{n}=286\end{array}$} & \multirow{2}{*}{$\begin{array}{c}\text { Statistical test } \\
\text { Chi square (Yates) } \\
\text { Relative risk (95\% CI) }\end{array}$} \\
\hline & No. & $\% / \mathrm{SD}$ & No. & $\% / \mathrm{SD}$ & \\
\hline Age (mean \& SD) & 30 & 5.5 & 25 & 5.5 & ANOVA $p<0.001$ \\
\hline *Past medical history & 26 & 27 & 54 & 19 & $2.50 \mathrm{p}=0.114)$ \\
\hline Less than traditional antenatal care & 24 & 30 & 73 & 33 & $0.05 \mathrm{p}=0.825$ \\
\hline Prodromal symptoms & 70 & 73 & 157 & 56 & $\begin{array}{c}7.98 \mathrm{p}=0.005 \\
1.31(1.11-1.53)\end{array}$ \\
\hline Prodromal signs (seen within 7 days) & 78 & 93 & 211 & 88 & $0.28 p=0.258$ \\
\hline $\begin{array}{l}\text { Diastolic BP immediately before } \\
\text { seizure (mean \& SD) }\end{array}$ & 96 & 16.1 & 97 & 14.0 & $\dagger 0.073 \mathrm{p}=0.786$ \\
\hline Maximum diastolic BP (mean \& SD) & 117 & 14.3 & 115 & 11.9 & $\dagger 0.54 \mathrm{p}=0.464$ \\
\hline Antepartum & 35 & 37 & 111 & 39 & $0.08 \mathrm{p}=0.772$ \\
\hline Preterm seizures & 39 & 41 & 129 & 45 & $0.42 \mathrm{p}=0.518$ \\
\hline First fit outside of hospital & 16 & 17 & 70 & 24 & $2.08 \mathrm{p}=0.149$ \\
\hline Multiple seizures & 38 & 40 & 120 & 42 & $0.12 \mathrm{p}=0.735$ \\
\hline Delivery by caesarean section & 46 & 48 & 159 & 56 & $1.41 \mathrm{p}=0.235$ \\
\hline Admission to intensive care unit & 47 & 49 & 124 & 43 & $0.70 \mathrm{p}=0.668$ \\
\hline Major maternal complication & 40 & 42 & 95 & 33 & $1.89 p=0.169$ \\
\hline Maternal mortality & 3 & 3 & 4 & 1 & $\ddagger \mathrm{p}=0.245$ \\
\hline GP reported maternal morbidity & 50 & 66 & 143 & 62 & $0.18 p=0.668$ \\
\hline $\begin{array}{l}\text { Small for gestational age fetus } \\
\text { (amongst } 356 \text { singletons) }\end{array}$ & 20 & 26 & 80 & 29 & $0.22 \mathrm{p}=0.639$ \\
\hline Fetal/infant mortality & 6 & 5 & 24 & 8 & $0.52 \mathrm{p}=0.471$ \\
\hline $\begin{array}{l}\text { Infant morbidity (amongst surviving } \\
\text { infants) }\end{array}$ & 16 & 17 & 53 & 21 & $0.75 p=0.385$ \\
\hline
\end{tabular}

This table includes 382 women with eclampsia (one woman was excluded due to missing data on parity). ANOVA - One way analysis of variance. *Past medical history includes a past history of diabetes, epilepsy, renal disease or hypertension. $\dagger-$ Kruskal-Wallis $\mathrm{H}$ test. $\ddagger$ - Fisher exact test. 


\subsection{ANTEPARTUM, INTRAPARTUM AND POSTPARTUM ECLAMPSIA.}

The results of the pilot study indicated that women with antepartum eclampsia had more maternal complications than women whose first fit occurred after the onset of labour. The numbers involved in the pilot study were very small so it was not possible to be confident that this finding was significant. The following hypothesis was formulated after completion of the pilot study and tested in the main study which involved a larger number of women.

5.5.1. Hypothesis arising from pilot study: Eclampsia occurring prior to labour is a more severe form of eclampsia than intra- or postpartum eclampsia.

\subsubsection{Results.}

Initial analysis was conducted on each of the three subgroups; antepartum, intrapartum and postpartum eclampsia. The intrapartum group was relatively small $(n=68)$ and very similar to the postpartum group. To simplify analysis and allow computation of relative risks further statistical analysis was conducted by comparing antepartum eclampsia with the combined intra- and postpartum group.

Women with antepartum, intrapartum and postpartum eclampsia did not differ significantly in terms of age, parity or past medical history. Antepartum eclampsia was more often associated with prodromal symptoms and less often with prodromal signs. Women with antepartum seizures had significantly higher diastolic blood pressures immediately before the seizure (taken within 1 hour of onset of seizures), and higher maximum diastolic blood pressures compared with women whose seizures occurred after the onset of labour. Women who developed convulsions prior to the onset of labour were significantly more likely to have their first fit outside of hospital, to have multiple convulsions, one or more major maternal complications and to be admitted to an intensive care unit. In this group there were also significantly more small for gestational age fetuses a higher fetal/infant mortality rate and a higher infant morbidity rate. The details are summarised in Table 5.6.

In Figure 4.4. it was shown that the type of eclampsia was closely associated with gestational maturity: most of the antepartum convulsions (76\% 111/147) occurred preterm and most of the intra- or postpartum seizures (75\% 178/236) presented at term. In view of this close relationship stratified analyses were performed to see which of the factors were independently associated with antepartum seizures. Antepartum eclampsia was associated with the presence of prodromal symptoms (relative risk (95\% confidence interval) 1.23 $(1.04-1.47))$ the absence of prodromal signs (relative risk $0.86(0.78-0.96))$, seizures outside of hospital (relative risk 36.24 (9.43 - 139.36)), delivery by Caesarean section 
(relative risk $2.29(1.90-2.76)$ ), multiple convulsions (relative risk $1.52(1.15-1.99))$, one or more major maternal complications (relative risk $1.63(1.21-2.19)$ ) admission to intensive care units (relative risk $1.43(1.12-1.84)$ ) and greater fetal/infant mortality (relative risk $3.27(1.23-8.68)$ ). All of these associations were significant independent of the gestation at which the seizure occurred (Table 5.6).

In summary in this study eclampsia occurring before the onset of labour was associated with more severe maternal consequences than eclampsia occurring after the onset of labour.

5.5.2. Prior hypothesis: Post-partum eclampsia which occurs without preexisting signs of severe pre-eclampsia is a relatively benign condition.

The above prior hypothesis was formulated from the impression of clinicians at the John Radcliffe Hospital that some women developed postpartum eclampsia without having established proteinuria and hypertension prior to delivery and that these women were relatively unaffected by their illness.

To test this hypothesis a group of women who developed eclampsia in the post-partum period without having documented proteinuria and hypertension prior to the onset of seizures was selected. This group of women was then compared with all other eclamptic women (i.e. antepartum, intrapartum eclampsia and postpartum eclampsia where there was established proteinuria and hypertension prior to the onset of seizures) in terms of maternal and fetal outcome measures.

\subsubsection{Results.}

On initial analysis the group without pre-seizure proteinuric hypertension were significantly different in several maternal and fetal outcome measures. They were less likely to require admission to intensive care, less likely to have a major maternal complication and their infants were less likely to be small for gestational age (Table 5.5.). These women were also significantly less likely to have preterm seizures which (as shown in section 5.6. below) is associated with poorer maternal and fetal outcomes. The data were therefore stratified for preterm and term onset of seizures and re-analysed to see if the differences in outcome still held once the fact that more of them occurred at term was accounted for. On stratification, all of the significant associations disappeared except for the fact that the women postpartum eclampsia without pre-fit proteinuric pre-eclampsia were significantly less likely to be admitted to an intensive care unit with a relative risk (95\% confidence interval) of 0.61 (0.39- 0.96). 
In summary in terms of maternal outcome measures, women who develop eclampsia postpartum without pre-existing proteinuria and hypertension seem to be less severely affected than other women. However once the fact that most of these seizures occur at term is accounted for most of the apparent differences cease to be statistically significant.

TABLE 5.5. Differences between postpartum eclampsia without pre-existing proteinuria and hypertension compared with all other eclampsia.

\begin{tabular}{|c|c|c|c|c|c|c|}
\hline \multirow[t]{2}{*}{ Factor of interest } & \multicolumn{2}{|c|}{$\begin{array}{c}\text { Postpartum } \\
\text { eclampsia } \\
\text { without pre-fit } \\
\text { pre-eclampsia } \\
\quad n=61\end{array}$} & \multicolumn{2}{|c|}{ Others } & \multirow[t]{2}{*}{$\begin{array}{c}\text { Statistical test } \\
\text { Chi square (Yates) } \\
\text { Relative risk }(95 \% \mathrm{CI})\end{array}$} & \multirow{2}{*}{$\begin{array}{c}\text { Results of stratified } \\
\text { analysis } \\
\text { M-H Sum. Chi Square } \\
\text { Sum. relative risk (95\% } \\
\text { CI) }\end{array}$} \\
\hline & No. & $\%$ & No. & $\%$ & & \\
\hline Preterm seizures & 8 & 13 & 153 & 49 & $\begin{array}{c}25.60 p<0.001 \\
0.27(0.14-0.51)\end{array}$ & NA \\
\hline Admission to intensive care unit & 16 & 26 & 148 & 48 & $\begin{array}{c}8.59 p=0.003 \\
0.55(0.36-0.85)\end{array}$ & $\begin{array}{c}4.85 p=0.028 \\
0.61(0.39-0.96)\end{array}$ \\
\hline Major maternal complication & 11 & 2 & 118 & 38 & $\begin{array}{c}8.07 p=0.005 \\
0.48(0.27-0.83)\end{array}$ & $\begin{array}{c}3.53 \mathrm{p}=0.060 \\
0.57(0.32-1.03)\end{array}$ \\
\hline Maternal mortality & 0 & 0 & 7 & 2 & ${ }^{*} \mathrm{p}=0.282$ & NA \\
\hline GP reported maternal morbidity & 28 & 62 & 160 & 63 & $0.00 \mathrm{p}=0.970$ & NA \\
\hline $\begin{array}{l}\text { Small for gestational age fetus } \\
\text { (amongst } 356 \text { singletons) }\end{array}$ & 4 & 8 & 93 & 32 & $\begin{array}{c}11.26 \mathrm{p}<0.001 \\
0.25(0.09-0.64)\end{array}$ & $\begin{array}{c}2.21 \mathrm{p}=0.137 \\
0.45(0.16-1.30)\end{array}$ \\
\hline Fetal/infant mortality & 1 & 1 & 28 & 9 & $3.46 \mathrm{p}=0.062$ & NA \\
\hline $\begin{array}{l}\text { Infant morbidity (amongst } \\
\text { surviving infants) }\end{array}$ & 6 & 10 & 59 & 21 & $3.05 p=0.08$ & NA \\
\hline
\end{tabular}

This table includes 372 cases (there were 11 cases where information on presence or absence of pre-fit preeclampsia was missing). * Fisher exact test. $\mathrm{NA}=$ Not applicable Relative risk $(95 \% \mathrm{CI})$ is the relative risk (95\% confidence interval) of the factor for woman with postpartum eclampsia without pre-fit pre-eclampsia. Stratified analysis - the tables were stratified for the gestation at fit (i.e. preterm or term). M-H Sum. Chi square = Mantel- Haenszel Summary Chi square. Sum relative risk $=$ the summary relative risk $(95 \%$ confidence interval) of the factor for women with postpartum eclampsia without pre-fit pre-eclampsia. 
TABLE 5.6. Differences between Antepartum and Intra- \& Postpartum Eclampsia.

\begin{tabular}{|c|c|c|c|c|c|c|}
\hline \multirow[t]{2}{*}{ Factor of interest } & \multicolumn{2}{|c|}{$\begin{array}{l}\text { Antepartum } \\
\qquad n=147\end{array}$} & \multicolumn{2}{|c|}{$\begin{array}{c}\text { Intrapartum \& } \\
\text { Postpartum } \\
n=236\end{array}$} & \multirow{2}{*}{$\begin{array}{c}\text { Statistical } \\
\text { significance } \\
\text { Chi square (Yates) } \\
\text { Relative risk (95\% CI) }\end{array}$} & \multirow{2}{*}{$\begin{array}{c}\text { Results of stratified } \\
\text { analysis } \\
\text { M-H Sum. Chi Square } \\
\text { Sum. relative risk (95\% } \\
\text { CI) }\end{array}$} \\
\hline & No. & $\% / S D$ & No. & $\% /$ SD & & \\
\hline Mean age in years (mean \& SD) & 26 & 5.9 & 26 & 6.0 & ANOVA $p=0.591$ & NA \\
\hline *Past medical history & 35 & 24 & 45 & 19 & $1.13 p=0.287$ & NA \\
\hline Primiparae & 111 & 76 & 175 & 74 & $0.08 \mathrm{p}=0.772$ & NA \\
\hline $\begin{array}{l}\text { Less than traditional antenatal } \\
\text { care }\end{array}$ & 43 & 36 & 54 & 29 & $1.42 \mathrm{p}=0.233$ & NA \\
\hline Prodromal symptoms & 107 & 75 & 120 & 51 & $\begin{array}{c}20.79 p<0.001 \\
1.48(1.26-1.73)\end{array}$ & $\begin{array}{c}5.09 p=0.024 \\
1.23(1.04-1.47)\end{array}$ \\
\hline $\begin{array}{l}\text { Prodromal signs } \\
\text { (seen within last } 7 \text { days) }\end{array}$ & 76 & 83 & 213 & 91 & $\begin{array}{c}4.34 p=0.037 \\
0.90(0.82-1.00)\end{array}$ & $\begin{array}{c}7.30 \mathrm{p}=0.006 \\
0.87(0.78-0.96)\end{array}$ \\
\hline $\begin{array}{l}\text { Diastolic BP immediately before } \\
\text { seizure (mean \& SD) }\end{array}$ & 105 & 14.2 & 95 & 13.9 & $\begin{array}{l}\text { Kruskal Wallis } \\
\text { H } 18.1 \text { p }<0.001\end{array}$ & $\dagger$ \\
\hline $\begin{array}{l}\text { Maximum diastolic BP } \\
\text { (mean \& SD) }\end{array}$ & 120 & 13.0 & 113 & 11.6 & $\begin{array}{l}\text { Kruskal Wallis } \\
\text { H } 26.48 \text { p }<0.001\end{array}$ & $\dagger$ \\
\hline Preterm seizures & 111 & 76 & 58 & 25 & $\begin{array}{c}93.26 p<0.001 \\
3.07(2.41-3.91)\end{array}$ & NA \\
\hline Fit outside hospital & 81 & 56 & 5 & 2 & $\begin{array}{c}14.2 p<0.001 \\
26.19(10.87-63.09)\end{array}$ & $\begin{array}{c}102.94 \mathrm{p}<0.001 \\
36.24(9.43-139.36)\end{array}$ \\
\hline Multiple seizures & 78 & 54 & 80 & 34 & $\begin{array}{c}13.84 p<0.001 \\
1.59(1.26-2.00)\end{array}$ & $\begin{array}{c}8.29 p=0.004 \\
1.52(1.15-1.99)\end{array}$ \\
\hline Delivered by caesarean section & 135 & 92 & 71 & 30 & $\begin{array}{c}136.49 p<0.001 \\
3.05(2.50-3.73)\end{array}$ & $\begin{array}{c}90.84 p<0.001 \\
2.29(1.90-2.76)\end{array}$ \\
\hline Admission to intensive care unit & 85 & 58 & 87 & 37 & $\begin{array}{c}15.25 p<0.001 \\
1.57(1.26-1.95)\end{array}$ & $\begin{array}{c}7.43 \mathrm{p}=0.006 \\
1.43(1.12-1.84)\end{array}$ \\
\hline Major maternal complication & 74 & 51 & 61 & 26 & $\begin{array}{c}23.3 \mathrm{p}<0.001 \\
1.96(1.50-2.57)\end{array}$ & $\begin{array}{c}6.56 p=0.010 \\
1.63(1.21-2.19)\end{array}$ \\
\hline Maternal death & 3 & 2 & 4 & 2 & $\ddagger \mathrm{p}=0.546$ & NA \\
\hline GP reported maternal morbidity & 84 & 69 & 110 & 60 & $2.40 \mathrm{p}=0.121$ & NA \\
\hline $\begin{array}{l}\text { Small for gestational age fetuses } \\
\text { (356 singletons only) }\end{array}$ & 62 & 44 & 38 & 18 & $\begin{array}{c}26.26 p<0.001 \\
2.42(1.72-3.42)\end{array}$ & $\begin{array}{c}1.27 \mathrm{p}=0.260 \\
1.25(0.87-1.79)\end{array}$ \\
\hline Total fetal/infant mortality & 24 & 16 & 6 & 2 & $\begin{array}{c}24.80 p<0.001 \\
7.03(2.94-16.82)\end{array}$ & $\begin{array}{c}5.82 \mathrm{p}=0.016 \\
3.27(1.23-8.68)\end{array}$ \\
\hline $\begin{array}{l}\text { Infant morbidity (amongst } \\
\text { surviving infants) }\end{array}$ & 41 & 36 & 28 & 12 & $\begin{array}{c}25.23 p<0.001 \\
2.94(1.92-4.50) \\
\end{array}$ & $\begin{array}{c}3.57 \mathrm{p}=0.059 \\
1.55(1.00-2.41) \\
\end{array}$ \\
\hline
\end{tabular}

SD = Standard deviation, NA = Not applicable *Past medical history includes a history of diabetes, epilepsy, renal disease or hypertension. $\ddagger$ - Fisher exact test. Relative risk $(95 \% \mathrm{CI})$ is the relative risk $(95 \% \mathrm{CI})$ of the factor for woman with antepartum eclampsia. Stratified analysis - tables were stratified for the gestation at fit (i.e. preterm or term). M-H Sum. Chi square = Mantel- Haenszel Summary Chi square. Sum relative risk = summary relative risk $(95 \% \mathrm{CI})$ of the factor for women with antepartum eclampsia. † Kruskal-Wallis $\mathrm{H}$ test Women with antepartum seizures occurring preterm had significantly higher diastolic blood pressures immediately before the fit and significantly higher maximum diastolic blood pressures than women with intra\& postpartum seizures occurring preterm. When eclampsia occurred at term there was no significant difference between antepartum and intra- \& postpartum seizures with regard to the diastolic blood pressures. 


\subsection{EARLY ONSET PRE-ECLAMPSIA - IS IT A MORE SEVERE ILLNESS?}

5.6.1. Hypothesis arising from pilot study: Pre-term eclampsia has more severe consequences for mother and fetus than eclampsia which occurs at term.

\subsubsection{Results.}

Forty-four percent (169) of women with eclampsia had their first seizure preterm (i.e. before 37 completed weeks of gestation), the remainder occurred at term. Preterm and term cases were compared.

Maternal age, parity and antenatal care were similar in the preterm and term groups. Women with preterm eclampsia were more likely to report prodromal symptoms and to have a past medical history of diabetes, epilepsy, hypertension or auto-immune disorders. There was no significant differences in the presence of prodromal signs (see Table 5.8) however women with preterm eclampsia did have significantly higher diastolic blood pressures immediately before the onset of seizures (i.e. taken within one hour of the onset of seizures) and significantly higher maximum recorded diastolic blood pressures.

Women with preterm eclampsia were more severely affected than those presenting at or after term in respect to multiple seizures, major maternal complications and admission to intensive care (Table 5.8.). There were no significant differences in general practitioner reported maternal morbidity or maternal death rates (although the very small number of deaths mean that there was limited power to detect any difference). These women had significantly more small for gestational age fetuses, more fetal/infant deaths and more infant morbidity.

The data were stratified to take into account the relationship between type of eclampsia and gestational age at presentation, and re-analysed to determine which factors were independently associated with preterm seizures. Preterm eclampsia was independently associated with; the presence of prodromal symptoms (relative risk (95\% confidence interval) $1.42(1.18-1.71)$, delivery by Caesarean section (relative risk $1.43(1.22-1.67)$ and the presence of one or more major maternal complications (relative risk 1.38 (1.02 1.85). Preterm eclampsia was also associated with small for gestational age fetuses (relative risk 4.18 (2.49 - 7.03) higher fetal/infant mortality rates (relative risk 16.91 (1.98 - 144.29) and higher rates of infant morbidity in surviving infants (relative risk 5.73 (2.90 - 11.35). All these associations were independent of the timing of the seizure in relation to the onset of labour (Table 5.8.). 
In summary, preterm eclampsia is associated with small for gestational age fetuses and higher fetal/infant mortality and morbidity. In addition preterm eclampsia appears to be associated with higher rates of maternal complications than eclampsia occurring at term.

5.6.2. Prior hypothesis: A significant proportion of eclampsia occurs in women at or before 32 weeks gestation.

\subsubsection{Results.}

Over one fifth $(21 \%-81)$ of the women developed eclampsia before 31 completed weeks. The earlier the onset of eclampsia the more severe the consequences appeared to be (Table 5.7.). This was true in terms of fetal/infant mortality and morbidity and also in terms of the number of fetuses that were small for gestational age and the number of mothers with major maternal complications. These trends were still significant even when data was stratified for the type of eclamptic seizure (i.e. antepartum versus intra- or postpartum seizures).

In summary, the hypothesis was upheld in that twenty-one percent of all eclampsia occurred at or before 32 weeks gestation, and the early onset was associated with poorer outcomes.

TABLE 5.7. Summary of differences in maternal and fetal outcome measures in women with very preterm, preterm and term eclampsia.

\begin{tabular}{|c|c|c|c|c|c|c|c|c|}
\hline \multirow[t]{2}{*}{ Factor of interest } & \multicolumn{2}{|c|}{$\begin{array}{c}<32 \text { weeks } \\
n=81\end{array}$} & \multicolumn{2}{|c|}{$\begin{array}{c}32 \text { - } 36 \text { weeks } \\
n=88\end{array}$} & \multicolumn{2}{|c|}{$\begin{array}{c}\geq \mathbf{3 7} \text { weeks } \\
n=214\end{array}$} & \multirow{2}{*}{$\begin{array}{l}\text { Statistical test } \\
\text { Chi square for } \\
\text { linear } \\
\text { trend }(2 \mathrm{df}) \\
\end{array}$} & \multirow{2}{*}{$\begin{array}{c}\text { Results of } \\
\text { stratified analysis } \\
\text { Chi square for } \\
\text { linear } \\
\text { trend }(2 \mathrm{df}) \\
\end{array}$} \\
\hline & No. & $\%$ & No. & $\%$ & No. & $\%$ & & \\
\hline Antepartum & 66 & 82 & 45 & 51 & 36 & 17 & $111.73 p<0.001$ & NA \\
\hline Admission to intensive care unit & 51 & 63 & 40 & 46 & 81 & 38 & $14.32 p<0.001$ & $0.56 \mathrm{p}=0.456$ \\
\hline Major maternal complication & 46 & 58 & 33 & 38 & 56 & 26 & $24.66 p<0.001$ & $7.70 \mathrm{p}=0.006$ \\
\hline Maternal mortality & 2 & 3 & 2 & 2 & 3 & 1 & $0.46 \mathrm{p}=0.499$ & NA \\
\hline GP reported maternal morbidity & 51 & 79 & 44 & 62 & 99 & 59 & $5.74 \mathrm{p}<0.017$ & $3.24 p=0.0718$ \\
\hline $\begin{array}{l}\text { Small for gestational age fetus } \\
\text { (amongst } \mathbf{3 5 6} \text { singletons) }\end{array}$ & 44 & 57 & 34 & 44 & 22 & 11 & $66.61 p<0.001$ & $39.94 p<0.001$ \\
\hline Fetal/infant mortality & 24 & 29 & 4 & 4 & 2 & 1 & $61.32 p<0.001$ & $29.97 \mathrm{p}<0.001$ \\
\hline $\begin{array}{l}\text { Infant morbidity (amongst } \\
\text { surviving infants) }\end{array}$ & 35 & 67 & 21 & 24 & 13 & 6 & $90.93 p<0.001$ & $62.13 \mathrm{P}<0.008$ \\
\hline
\end{tabular}

Table includes all 383 women with eclampsia. SD - Standard deviation. NA - Not applicable. Stratified analysis - the tables were stratified for the type of fit (i.e. antepartum eclampsia or eclampsia occurring after the onset of labour). 
TABLE 5.8. Differences between preterm and term eclampsia.

\begin{tabular}{|c|c|c|c|c|c|c|}
\hline \multirow{2}{*}{ Factor of interest } & \multicolumn{2}{|c|}{$\begin{array}{l}\text { Preterm } \\
n=169\end{array}$} & \multicolumn{2}{|c|}{$\begin{array}{l}\text { Term } \\
\mathrm{n}=214\end{array}$} & \multirow{2}{*}{$\begin{array}{c}\text { Statistical } \\
\text { significance } \\
\text { Chi square (Yates) } \\
\text { Relative risk }(95 \% \mathrm{CI})\end{array}$} & \multirow{2}{*}{$\begin{array}{c}\text { Results of stratified } \\
\text { analysis } \\
\text { M-H Sum. Chi Square } \\
\text { Sum. relative risk (95\% } \\
\text { CI) }\end{array}$} \\
\hline & No. & $\% / \mathrm{SD}$ & No. & $\% / \mathrm{SD}$ & & \\
\hline Mean age in years (mean \& SD) & 26 & 5.6 & 26 & 6.3 & ANOVA $\mathrm{p}=0.800$ & NA \\
\hline *Past medical history & 44 & 27 & 36 & 17 & $\begin{array}{c}4.61 \mathrm{p}=0.032 \\
1.57(1.06-2.32)\end{array}$ & $\begin{array}{c}3.20 \mathrm{p}=0.074 \\
1.54(0.99-2.37)\end{array}$ \\
\hline Primiparae & 129 & 77 & 157 & 73 & $0.42 \mathrm{p}=0.518$ & NA \\
\hline $\begin{array}{l}\text { Less than traditional antenatal } \\
\text { care }\end{array}$ & 45 & 33 & 52 & 31 & $0.10 \mathrm{p}=0.751$ & NA \\
\hline Prodromal symptoms & 124 & 76 & 103 & 48 & $\begin{array}{c}29.05 \mathrm{p}<0.001 \\
1.58(1.34-1.86)\end{array}$ & $\begin{array}{c}13.29 p<0.001 \\
1.42(1.18-1.71)\end{array}$ \\
\hline $\begin{array}{l}\text { Prodromal signs } \\
\text { (seen within last } 7 \text { days) }\end{array}$ & 113 & 90 & 176 & 88 & $0.24 \mathrm{p}=0.625$ & NA \\
\hline $\begin{array}{l}\text { Diastolic BP immediately before } \\
\text { seizure (mean \& SD) }\end{array}$ & 101 & 13.9 & 95 & 21.2 & $\begin{array}{l}\text { Kruskal Wallis } \\
\mathrm{H}=8.76 \mathrm{p}=0.003\end{array}$ & NSD \\
\hline $\begin{array}{l}\text { Maximum diastolic BP } \\
\text { (mean \& SD) }\end{array}$ & 119 & 12.7 & 113 & 11.7 & $\begin{array}{l}\text { Kruskal Wallis } \\
\mathrm{H}=23.63 \mathrm{p}<0.001\end{array}$ & $\dagger$ \\
\hline Antepartum seizures & 111 & 66 & 36 & 17 & $\begin{array}{c}96.08 \mathrm{p}<0.001 \\
3.94(2.87-5.41)\end{array}$ & NA \\
\hline Fit outside of hospital & 64 & 38 & 22 & 10 & $\begin{array}{c}40.16 p<0001 \\
3.71(2.39-5.76)\end{array}$ & $\begin{array}{c}0.20 p=0.656 \\
1.12(0.76-1.65)\end{array}$ \\
\hline Multiple seizures & 81 & 49 & 77 & 36 & $\begin{array}{c}5.55 p=0.018 \\
1.35(1.06-1.71)\end{array}$ & $\begin{array}{c}0.29 \mathrm{p}=0.589 \\
1.10(0.83-1.44)\end{array}$ \\
\hline Delivered by caesarean section & 134 & 79 & 72 & 34 & $\begin{array}{c}77.32 \mathrm{p}<0.001 \\
2.36(1.92-2.89)\end{array}$ & $\begin{array}{c}17.64 p<0.001 \\
1.43(1.22-1.67)\end{array}$ \\
\hline Admission to intensive care unit & 91 & 54 & 81 & 38 & $\begin{array}{c}9.13 p=0.003 \\
1.42(1.14-1.77)\end{array}$ & $\begin{array}{c}1.44 \mathrm{p}=0.230 \\
1.18(0.92-1.52)\end{array}$ \\
\hline Major maternal complication & 79 & 47 & 56 & 26 & $\begin{array}{c}17.01 p<0.001 \\
1.80(1.36-2.37)\end{array}$ & $\begin{array}{c}3.94 p=0.047 \\
1.38(1.02-1.85)\end{array}$ \\
\hline Maternal death & 4 & 2 & 3 & 1 & $\ddagger \mathrm{p}=0.372$ & NA \\
\hline GP reported maternal morbidity & 95 & 69 & 99 & 59 & $3.01 \mathrm{p}=0.083$ ) & NA \\
\hline $\begin{array}{l}\text { Small for gestational age fetuses } \\
\text { (356 singletons only) }\end{array}$ & 78 & 50 & 22 & 11 & $\begin{array}{c}63.92 p<0.001 \\
4.53(2.96-6.92)\end{array}$ & $\begin{array}{c}40.72 \mathrm{p}<0.001 \\
4.18(2.49-7.03)\end{array}$ \\
\hline Fetal/Infant mortality & 28 & 15 & 2 & 1 & $\begin{array}{c}29.67 \mathrm{p}<0.001 \\
17.79(4.29-73.69)\end{array}$ & $\begin{array}{c}12.60 \mathrm{p}<0.001 \\
16.91(1.98-144.29)\end{array}$ \\
\hline $\begin{array}{l}\text { Infant morbidity } \\
\text { (amongst surviving infants) }\end{array}$ & 56 & 40 & 13 & 6 & $\begin{array}{c}57.08 \mathrm{p}<0.001 \\
6.32(3.60-11.11) \\
\end{array}$ & $\begin{array}{c}37.01 \mathrm{p}<0.001 \\
5.73(2.90-11.35) \\
\end{array}$ \\
\hline
\end{tabular}

Table includes all 383 women with eclampsia. *Past medical history includes a history of diabetes, epilepsy, renal disease or hypertension. $\ddagger$ - Fisher exact test. SD - Standard deviation. NA - Not applicable. NSD - No significant difference. Relative risk $(95 \% \mathrm{CI})$ is the relative risk $(95 \% \mathrm{CI})$ of the factor for woman with preterm eclampsia. Stratified analysis - tables were stratified for the type of fit (i.e. antepartum eclampsia or intra- or post partum). M-H Sum. Chi square $=$ Mantel- Haenszel Summary Chi square. Sum relative risk $=$ summary relative risk $(95 \% \mathrm{CI})$ of the factor for women with preterm eclampsia. $\dagger$ - Kruskal Wallis test - women with preterm seizures had significantly higher maximum diastolic blood pressures than women with term seizures independent of type of fit. 


\subsection{LOGISTIC REGRESSION ANALYSIS.}

The previous analyses involved the testing of prior hypotheses and a number of the variables were found to be associated with poorer maternal or fetal outcomes such as; maternal death, the presence of major maternal complications, general practitioner recorded maternal morbidity, fetal death and fetal morbidity. It became clear that preterm and antepartum fits amongst other variables were associated with each outcome as well as with each other. Stratification of tables was done to try to clarify which variables were independently associated with the outcomes but stratification of more than one level was not possible as the number of cases in each cell of the tables became too small to conduct meaningful statistical analyses. In this complex situation multiple logistic regression analysis was used as an analytic tool in an effort to assess which variables were important in contributing to the outcomes of interest, .

\subsubsection{Introduction to multiple logistic regression analysis.}

There were only seven maternal deaths in the study and this number was too small to allow meaningful multiple regression analysis. For the remaining 4 outcome variables multiple logistic regression analysis was conducted using SPSS for Windows and the statistical method outlined in section 3.8.2.1.

In each regression analysis selection of candidate dependent variables was based on biological plausibility and the previously described univariate analysis (Altman.D, 1991). The variables, their type and code are shown in Table 5.9.

Odds ratios were calculated for each independent variable. Given the limitations of the logistic regression model and the frequency of the occurrence of the outcome variables in this population the odds ratios shown can not be considered to be valid estimates of the relative risk. However they can be used as indicators of the relative importance of each of the independent variables controlling for the contribution of each of the other variables in the model. 
TABLE 5.9. Variables, their type and coding system used in multiple logistic regression analyses.

\begin{tabular}{|c|c|c|}
\hline Variables & Type & Coding system used \\
\hline Maternal age & Categorical & $0=20-34$ yrs $1=<20$ yrs $2=\geq 35 \mathrm{yrs}$ \\
\hline Past medical history & Binary & $0=$ none $1=$ significant past history \\
\hline Previous pregnancy & Binary & $0=$ primiparous $1=$ multiparous \\
\hline Multiple pregnancy & Binary & $0=$ singleton $1=$ multiple pregnancy \\
\hline No antenatal care & Binary & $0=$ some antenatal care $1=$ no antenatal care \\
\hline First recorded diastolic BP & Continuous & $\begin{array}{l}\text { First diastolic BP minus the median first diastolic BP } \\
(70)\end{array}$ \\
\hline First recorded systolic BP & Continuous & First systolic BP minus the median first systolic BP (110) \\
\hline Pre fit proteinuric pre-eclampsia & Binary & $\begin{array}{l}0=\text { none } 1=\text { proteinuric pre-eclampsia recorded prior to } \\
\text { fit }\end{array}$ \\
\hline Maximum recorded diastolic $\mathrm{BP}$ & Continuous & $\begin{array}{l}\text { Maximum diastolic BP minus the median maximum } \\
\text { diastolic BP (115) }\end{array}$ \\
\hline Maximum recorded systolic BP & Continuous & $\begin{array}{l}\text { Maximum systolic BP minus the median maximum } \\
\text { systolic BP (180) }\end{array}$ \\
\hline Change in diastolic BP & Continuous & $\begin{array}{l}\text { Change in diastolic BP minus the median change } \\
\text { diastolic BP (48) }\end{array}$ \\
\hline Change in systolic BP & Continuous & $\begin{array}{l}\text { Change systolic BP minus the median change systolic BP } \\
(65)\end{array}$ \\
\hline Maximum recorded proteinuria & Categorical & $0=$ none, trace or,$+ 1=++, 2=+++3=++++$ \\
\hline Location of fit & Binary & $0=$ hospital $1=$ elsewhere \\
\hline Multiple seizures & Binary & $0=$ single seizure $1=$ multiple seizures \\
\hline Type of fit & Binary & $0=$ intra- or postpartum seizure $1=$ antepartum seizure \\
\hline Gestation of fit & Binary & $0=$ term seizure $1=$ preterm seizure \\
\hline Symptoms & Binary & $0=$ no symptoms $1=$ symptomatic \\
\hline Method of delivery & Binary & $0=$ vaginal delivery $1=$ caesarean section \\
\hline Small for gestational age fetus & Binary & $0=\geq 10^{\text {th }}$ centile $1=<10^{\text {th }}$ centile for gestational age \\
\hline Location of fit $x$ multiple fits & Interaction & \\
\hline Type of fit $x$ gestation of fit & Interaction & \\
\hline $\begin{array}{l}\text { Type of fit } x \text { small for gestational } \\
\text { age fetus }\end{array}$ & Interaction & \\
\hline $\begin{array}{l}\text { Gestation at fit } x \text { small for } \\
\text { gestational age fetus }\end{array}$ & Interaction & \\
\hline Major maternal complication & Binary & $0=$ none $1=$ at least one \\
\hline $\begin{array}{l}\text { General practitioner reported } \\
\text { morbidity }\end{array}$ & Binary & $0=$ none $1=$ at least some \\
\hline Fetal/infant death & Binary & $0=$ living fetus/infant $1=$ death of fetus/infant \\
\hline Infant morbidity & Binary & $0=$ none $1=$ at least some \\
\hline
\end{tabular}




\subsubsection{Associations with major maternal complications.}

Overall $135(35 \%)$ women developed at least one major complication following their eclamptic seizures. The types of complications considered major are listed in Table 4.9. The variables tested for inclusion in the model with major maternal complications as the dependent variable were: Maternal age, Past medical history, Previous pregnancy, Multiple pregnancy, No antenatal care, Pre-fit proteinuric pre-eclampsia, First recorded diastolic blood pressure, First recorded systolic blood pressure, Maximum recorded diastolic blood pressure, Maximum recorded systolic blood pressure, Change in diastolic blood pressure, Change in systolic blood pressure, Maximum recorded proteinuria, Location of fit, Multiple seizures, Type of fit, Gestation of fit, Symptoms (headaches, epigastric pain, visual disturbance), Small for gestational age fetus/infant, Location of fit x Multiple fits and Type of fit $\mathrm{x}$ Gestation of fit.

\subsubsection{Results}

Table 5.10. shows parameters for those variables that were incorporated into the model. For the blood pressure variables (i.e. continuous variable) the odds ratio shown represents the odds for a change of $5 \mathrm{~mm} \mathrm{Hg}$ from the median value. Thus the variables that were independently associated with major maternal complications were multiple seizures, antepartum seizures, maximum recorded diastolic blood pressure and maximum recorded systolic blood pressure. None of the other variables listed above met the inclusion criteria specified for the model, suggesting that the predisposition to major maternal complications is best "explained" by the combination of these four predictor variables.

TABLE 5.10. Multiple logistic regression model of variables independently associated with major maternal complications.

\begin{tabular}{|c|c|c|c|c|}
\hline Variables included in the model & Coeff & S.E. & $\mathbf{P}$ & Odds ratio $(95 \% \mathrm{CI})$ \\
\hline Multiple fit & 1.0123 & 0.2549 & 0.0001 & $2.75(1.67-4.54)$ \\
\hline Type of fit & 0.7568 & 0.2601 & 0.0036 & $2.13(1.28-3.55)$ \\
\hline $\begin{array}{l}\text { Maximum diastolic blood pressure } \\
\text { (median } 115 \mathrm{~mm} \mathrm{Hg} \text { ) }\end{array}$ & 0.0379 & 0.0142 & 0.0076 & $1.21(1.05-1.39)^{*}$ \\
\hline $\begin{array}{l}\text { Maximum systolic blood pressure } \\
\text { (median } 180 \mathrm{~mm} \mathrm{Hg} \text { ) }\end{array}$ & 0.0146 & 0.0072 & 0.0425 & $1.08(0.71-1.63)^{*}$ \\
\hline Constant & -1.4796 & 0.1988 & 0.0000 & \\
\hline
\end{tabular}

Number of cases included in analysis $=349$ (383 selected and 34 cases rejected due to missing data)

* Odds ratio shown $=$ odds for change of $5 \mathrm{~mm} \mathrm{Hg}$ from the median value 


\subsubsection{Associations with general practitioner reported maternal morbidity.}

One hundred and ninety four (63\%) of the 306 women whose general practitioners responded had some documented post eclamptic morbidity. The variables tested for inclusion in the regression model for general practitioner reported morbidity were: Maternal age, Past medical history, Previous pregnancy, Multiple pregnancy, No antenatal care, First recorded diastolic blood pressure, First recorded systolic blood pressure, Pre fit proteinuric pre-eclampsia, Maximum recorded diastolic blood pressure, Maximum recorded systolic blood pressure, Change in diastolic blood pressure, Change in systolic blood pressure, Maximum recorded proteinuria, Location of fit, Multiple seizures, Type of fit, Gestation of fit, Symptoms, Method of delivery, Major maternal complication, Small for gestational age fetus, Fetal/infant death, Location of fit $\mathrm{x}$ multiple fits and Type of fit $\mathrm{x}$ Gestation of fit.

\subsubsection{Results}

The final multiple regression model for variables associated with general practitioner reported maternal morbidity are shown in Table 5.11. There were 280 cases included in the analysis. One hundred and three cases were excluded either because the general practitioner questionnaire was not returned or there was other missing data.

The variables associated with the presence or absence of maternal morbidity are fetal/infant death, major maternal complications, the first systolic blood pressure, and the maximum systolic blood pressure.

TABLE 5.11. Final multiple logistic regression model of variables associated with general practitioner reported maternal morbidity.

\begin{tabular}{|c|c|c|c|c|}
\hline Variables included in the model & Coeff & S.E. & $\mathbf{P}$ & Odds ratio $(95 \% \mathrm{CI})$ \\
\hline Fetal/infant death & 1.3179 & 0.6439 & 0.0407 & $3.74(1.03-13.2)$ \\
\hline Major maternal complication & 0.6275 & 0.2943 & 0.0330 & $1.87(1.05-3.33)$ \\
\hline $\begin{array}{l}\text { First systolic blood pressure } \\
\text { (median }=110 \mathrm{~mm} \mathrm{Hg} \text { ) }\end{array}$ & 0.0331 & 0.0110 & 0.0026 & $1.18(1.05-1.31)^{*}$ \\
\hline $\begin{array}{l}\text { Maximum systolic blood pressure } \\
\text { (median }=180 \mathrm{~mm} \mathrm{Hg})\end{array}$ & 0.0127 & 0.0065 & 0.0493 & $1.07(1.00-1.14)^{*}$ \\
\hline Constant & 0.1461 & 0.1655 & 0.3775 & \\
\hline
\end{tabular}

Number of cases included in the analysis $=280$ (326 selected 46 excluded due to missing data)

* Odds ratio shown $=$ odds for change of $5 \mathrm{~mm} \mathrm{Hg}$ from the median value 


\subsubsection{Associations with fetal/infant death.}

The logistic regression analysis on fetal/infant death was carried out on the 356 singleton pregnancies. All thirty fetal/infant deaths occurred amongst singletons. The variables tested for inclusion in the regression model were: Maternal age, Past medical history, Previous pregnancy, No antenatal care, First recorded diastolic blood pressure, First recorded systolic blood pressure, Pre-fit proteinuric pre-eclampsia, Maximum recorded diastolic blood pressure, Maximum recorded systolic blood pressure, Change in diastolic blood pressure, Change in systolic blood pressure, Maximum recorded proteinuria, Location of fit, Multiple seizures, Type of fit, Gestation of fit, Symptoms, Major maternal complication, Small for gestational age fetus, Location of fit $\mathrm{x}$ multiple fits, Type of fit $\mathrm{x}$ Gestation of fit, Type of fit $\mathrm{x}$ small for gestational age fetus and Gestation at fit $\mathrm{x}$ small for gestational age fetus.

\subsubsection{Results}

The logistic regression analysis for variables associated with fetal/infant death was carried out in a forward stepwise manner as described above. The model produced included the variables Gestation of fit, No antenatal care and the interaction variable Gestation of fit $\mathrm{x}$ Type of fit as shown in Table 5.12.

Logistic regression analysis involves a hierarchical strategy which means that if an interaction term (i.e. Gestation of fit $x$ Type of fit) is included then the model should usually also include all component effects (i.e. both Gestation of fit and Type of fit). As this was not the case with the model shown in Table 5.12. the regression was repeated with forced entry of the variable Type of fit. When this fourth variable was included in the model the only variable which was still significantly associated with fetal/infant death was the Gestation of fit. After discussion with a statistician it was felt that the best model was still the original model shown above. The fact that Type of fit was not significant on its own but was in combination with Gestation of fit implies that antepartum seizures were not associated with higher rates of fetal/infant death on their own but that the combination of antepartum and preterm seizures was associated with higher rates of fetal/infant death. This seems plausible as a preterm fetus may not be able to cope with the period of relative hypoxia during a maternal antepartum seizure whereas a term infant (with greater reserves) may be unaffected by the same period of relative hypoxia. 
TABLE 5.12. Final multiple logistic regression model of variables associated with fetal/infant death.

\begin{tabular}{lcrrl}
\hline \multicolumn{1}{c}{ Variables included in the model } & Coeff & S.E. & P & \multicolumn{1}{c}{ Odds ratio (95\% CI) } \\
\hline Gestation of fit & 2.7462 & 1.1448 & 0.0165 & $15.6(1.65-147)$ \\
No antenatal care & 2.3795 & 1.4028 & 0.0898 & $10.8(0.69-169)$ \\
Gestation of fit x type of fit & 1.2757 & 0.6367 & 0.0451 & $55.8(1.70-1830)$ \\
Constant & -5.3260 & 1.0136 & 0.000 & \\
\hline
\end{tabular}

Number of cases included $=323$ ( 356 singleton pregnancies selected, 33 excluded due to missing data)

\subsubsection{Associations with infant morbidity - multiple logistic regression analysis}

Infant morbidity in this study was assessed by report in maternal case-notes, the general practitioner questionnaire or from the special care baby unit. Table 4.11. show the type of morbidity which was included and information was available on 278 of the 326 surviving singletons. The variables tested for inclusion in the multiple logistic regression model were: Maternal age, Past medical history, Previous pregnancy, No antenatal care, First recorded diastolic blood pressure, First recorded systolic blood pressure, Pre-fit proteinuric preeclampsia, Maximum recorded diastolic blood pressure, Maximum recorded systolic blood pressure, Change in diastolic blood pressure, Change in systolic blood pressure, Maximum recorded proteinuria, Location of fit, Multiple seizures, Type of fit, Gestation of fit, Symptoms, Major maternal complication, Small for gestational age fetus, Location of fit $\mathrm{x}$ multiple fits, Type of fit $\mathrm{x}$ gestation of fit, Type of fit $\mathrm{x}$ small for gestational age fetus and Gestation at fit $\mathrm{x}$ small for gestational age fetus

\subsubsection{Results}

The final logistic regression model of variables associated with infant morbidity is shown in Table 5.13. Pre-term infants and those born to women who had symptoms (headache, visual disturbance or epigastric pain) in association with eclampsia were significantly more likely to have morbidity reported.

TABLE 5.13. Final multiple logistic regression model of variables associated with infant morbidity.

\begin{tabular}{lcccc}
\hline \multicolumn{1}{c}{ Variables included in the model } & Coeff & S.E. & P & Odds ratio (95\% CI) \\
\hline Gestation at fit & 2.2677 & 0.3744 & 0.0000 & $9.65(4.64-20.1)$ \\
Symptomatic pre-eclampsia & 1.1854 & 0.4010 & 0.0031 & $3.27(1.49-7.17)$ \\
Constant & -3.3748 & 0.4240 & 0.0000 & \\
\hline
\end{tabular}

278 cases included in analysis (326 surviving singletons selected and 48 rejected due to missing data) 


\subsection{DISCUSSION.}

\subsubsection{Antenatal care.}

The role of antenatal care in pre-eclampsia and eclampsia is a matter for debate. As stated in Chapter 1 there is good evidence that the introduction of routine antenatal care was associated with a decrease in the incidence of eclampsia. However in developed countries with a high rate of antenatal care uptake there has been increasing discussion about the ideal frequency and content of antenatal visits (Backe \& Nakling, 1993; Hall et al. 1980) particularly in relation to the detection and management of women with pre-eclampsia (Hall \& Campbell, 1992; Wallenburg, 1989).

The supposed benefit of antenatal care is that it allows detection of early signs of preeclampsia so that with adequate intervention and management eclampsia can be avoided or at least its complications minimised. The underlying assumption is that prodromal symptoms and signs of eclampsia are always present if looked for frequently enough. The majority of all women in this study had intervals between checks on blood pressure of less than two weeks prior to developing eclampsia. Therefore the opportunity for early detection and intervention was still present.

When assessing the antenatal group alone, women with a long screening interval tended to have worse maternal and fetal outcomes compared with women being screened every fortnight or more frequently. They were significantly more likely to have their first fit outside of hospital and to have multiple seizures. In this relatively small sample all other differences failed to reach statistical significance but the trend was remarkably consistent. Screening all women at least once a fortnight may allow early intervention and reduce the number of antepartum seizures which occur outside of hospital and may even reduce the number of multiple antepartum seizures. However from this study it is not possible to conclude that frequent screening reduces maternal or fetal morbidity or mortality from antepartum seizures.

\subsubsection{Unheralded eclampsia.}

Clinicians who think in terms of the traditional model of pre-eclampsia/eclampsia (Figure 1.1.) use the presence and severity of hypertension and proteinuria to try to predict the onset of eclampsia. The analysis on unheralded eclampsia shows that if the presence of proteinuric hypertension is the sole criteria for predicting eclampsia, then even if women were screened every 24 hours $33 \%$ of eclamptic women would not be detected as being at risk before their first seizure. The clinical reality is that screening checks are less frequent 
than every 24 hours and the proportion who present with unheralded eclampsia is even larger (i.e. $48 \%$ of women in this study did not have recorded hypertension and proteinuria prior to their first fit).

There are two conclusions to be drawn from this. Firstly, it has to be accepted that using conventional screening procedures eclampsia will not always be predicted or prevented. It is therefore important to optimise the diagnosis and management of eclampsia so that when it does occur its effects are minimised.

The second conclusion is that to make progress in the prevention of eclampsia newer, more effective screening measures need to be found. If the current theory of pathogenesis of preeclampsia/eclampsia (Figure 1.2.) is accepted then hypertension and proteinuria are just two examples of the various systemic disturbances created by endothelial cell damage. When the factor which links the ischaemic placenta with endothelial cell damage is known it may be possible to screen for its presence. However this is not likely to become available in the next few years.

Although $33 \%$ of women did not have proteinuric hypertension prior to the onset of seizures $93 \%$ did have either proteinuria or hypertension before fitting. It seems that the presence of either hypertension or proteinuria should be used as an initial warning to signal further investigation looking for other signs of endothelial cell damage. Table 4.7. shows that biochemical parameters of systemic disturbances were not measured routinely even in women hospitalised before their first seizure.

\subsubsection{Morbidity in older women.}

Several studies in the past have reported increased maternal (Cummins, 1973; Porapakkham, 1979) and fetal (Neutra \& Neff, 1975) mortality of eclampsia in older women. At least some of this increased risk has been attributed to a greater rate of coincident illness such as chronic hypertension. In this study the small group of older women (35 or more years of age) were more likely to have a significant past medical history of a chronic condition but they did not fare any worse than the rest of the eclamptic women in terms of maternal or fetal outcomes. Several of the previous studies have come from developing countries and it could be postulated that the women were less likely to have access to effective medical care and therefore were more likely to have poorly controlled chronic illness. The past medical histories recorded in women in this study included a range of hypertension, diabetes, auto-immune disorders and epilepsy. Most of the women in this study were on medication and were stable at the time of eclampsia, hence their resilience to 
the insult of eclampsia may have varied little from women without a previously diagnosed chronic condition.

\subsubsection{Multiparous women.}

In recent population based studies of eclampsia the proportion of multiparous women has been reported to be between 20-30\% (Wightman et al. 1978; Moller \& Lindmark, 1986; Templeton \& Campbell, 1979) and the findings of this study are consistent with this.

There have previously been suggestions that multiparous women are at an increased risk of morbidity and mortality from eclampsia (Porapakkham, 1979). No such association was found in this study. There is no logical reason why multiparity itself should confer a greater risk of morbidity and mortality from eclampsia. However both grand multiparity and preexisting medical illness are known to increase susceptibility to increased morbidity in any pregnancy. In this study there were relatively few grand multiparae and the multiparous women had no greater incidence of significant past medical history (hypertension, epilepsy, renal failure, diabetes, auto immune disorders) compared to primiparous women which may explain why there were no significant differences in outcome.

In this study multiparous women were more likely to have reported symptoms prior to the onset of eclampsia. One possible explanation is that having experienced one pregnancy multiparous women knew what to expect and therefore were more likely to report unusual symptoms.

\subsubsection{Antepartum vs. intra- and postpartum convulsions.}

The results in Section 5.5. support the finding from previous studies (Odum \& Akinkugbe, 1991; Lopez Llera, 1992) that women who develop antepartum eclampsia have poorer maternal outcomes than those who develop it during or after labour. This study has taken into account the close relationship between preterm and antepartum seizures and the association of poorer maternal outcomes with antepartum seizures still stands.

The reasons for the poorer outcome are uncertain as there were no significant differences in the past medical histories or age of these women. The antepartum group did have significantly higher diastolic blood pressures prior to the onset of seizures and higher maximal blood pressures. This finding differs from that of Llopez Llera (Lopez Llera, 1992) and may indicate that the physical disturbances associated with the eclamptic seizures were more intense antepartum. The problems may have also been exacerbated by the fact 
that more of these women had multiple seizures, seizures at home (leading to delays in the institution of effective management) and more often were delivered by caesarean section.

Llopez Llera (1992) compared antepartum seizures with intra- and postpartum seizures and found poorer fetal as well as maternal outcomes in the antepartum group but he did not stratify to take account of the influence of gestational age. This study had similar findings prior to the stratification of data but once the effect of prematurity was controlled for, the apparent differences in fetal/infant mortality and small for gestational age infants disappeared. The surviving infants of mothers with antepartum seizures still had significantly more general practitioner reported morbidity than other infants in the study. This may be associated with the fact that infants were exposed to a period of relative hypoxia during the fit(s) or could reflect biases in the reporting to general practitioners by mothers who had severe disease themselves and gave birth prematurely.

It had been thought that women who developed eclampsia postpartum without a long prodromal period of pre-eclampsia would have fewer maternal complications than all other women. This arose from clinical observations and the postulated reasoning for it was that treatment would be initiated early and aggressively in these women as most were in hospital and there was no need to balance the needs of the fetus with the mother. In fact the group with postpartum eclampsia and no pre-existing proteinuria and hypertension fared no better than the others in terms of maternal outcomes. This finding may indicate that it is the seizures themselves that dictate maternal outcome rather than the extent or severity of the prodromal illness. Another possible explanation is that these women actually had delays in the initiation of appropriate management because their carers were hesitant about making the diagnosis of eclampsia when there had not been previous hypertension and proteinuria.

\subsubsection{Preterm vs. term.}

Prematurity has previously been well established as one of the major causes of poor perinatal outcome (Neutra, 1975; Sibai et al. 1983) and the findings in this study were consistent with this.

In Chapter 1 (Section 1.5.2.) studies which found higher fetal and maternal mortality and morbidity rates associated with preterm eclampsia (Moller \& Lindmark, 1986; Lopez Llera et al. 1976) were discussed. This study (considerably larger than previous studies) found no difference in maternal mortality or general practitioner reported morbidity in preterm and term groups. However women with preterm eclampsia had significantly more major maternal complications independent of the onset of seizures in relation to labour. 
The logistic regression analysis was done to try to clarify the relationships between the multiple independent variables and the major outcome variables.

\subsubsection{Major maternal complications}

From the subgroup analysis (Section 5.5.) it was observed that those women who had antepartum seizures were more likely to have at least one major maternal complication. The logistic regression analysis confirms this result while controlling for other potential confounders and shows that the presence of multiple seizures and high maximum diastolic and systolic blood pressures were also independently associated with the presence of major maternal complications.

The complications considered 'major' were shown in Table 4.9. Multiple seizures and very high blood pressures are plausible antecedents to these events. Multiple seizures indicate several periods of relative hypoxia and an increased risk of aspiration. Antenatal seizures mean that carers have to balance the needs of mother and fetus and there may be delays in management while fetal well-being is assessed which allows progression of the disease state. Finally it is well established that severe degrees of hypertension predispose to cerebrovascular accidents, renal failure and cortical blindness.

It is important to know whether rigorous blood pressure and seizure control could reduce the rate of major maternal complications. However a study aimed at testing this issue would be difficult to undertake in the developed world because of the relative rarity of eclampsia. The Report of the Confidential Enquiry into Maternal Deaths has repeatedly stressed the need for rigorous blood pressure control to prevent maternal death from eclampsia (DHSS \& Welsh Office. 1989; DHSS et al. 1991). The evidence of this study is that high blood pressure in eclampsia is independently associated with major complications.

\subsubsection{Maternal morbidity}

Table 4.10. shows the types of morbidity reported by general practitioners. The most common morbid feature reported by general practitioners was hypertension so it is unsurprising to find that high systolic blood pressures were associated with reported maternal morbidity. High first recorded systolic blood pressure (as well as maximum systolic blood pressure) was also associated which may indicate that the general practitioners were reporting on a re-presentation of an old problem of hypertension. 
Many of the complaints included in Table 4.10. such as anxiety, headaches and depression are associated with grief reactions. It is therefore understandable that these symptoms would be more common after either a fetal/infant death or a major maternal illness.

\subsubsection{Fetal/infant deaths}

The analysis of the 30 fetal/infant deaths should be viewed with caution as there were several factors which may have biased the analysis. First, in this analysis, all of the fetal/infant deaths have been considered together, although there may be different factors associated with intrauterine fetal deaths compared with neonatal deaths. Separate consideration of the factors involved in second trimester miscarriages, stillbirths and neonatal deaths could not be done as each individual group was too small. Second, the study involved review of the maternal case-notes only, therefore information on the infants was not always complete. As described in Chapter 4 there were 7 cases of infants admitted to special care nurseries where it was not confirmed that they were discharged alive. These infants were included as survivors in this analysis although some may have succumbed in the late neonatal period.

It is well established that preterm birth is associated with higher rates of fetal and infant deaths in eclampsia (Neutra, 1975; Sibai et al. 1983) and this study is consistent with the previous findings. The complete lack of any antenatal care was also associated with a trend (although it was not statistically significant $p=0.09$ ) to increased risk of fetal/infant mortality. As there were only 13 women who had no antenatal care this result must be viewed with caution.

Unlike previous studies (Neutra \& Neff, 1975) birth weight less than the tenth centile for gestational age was not found to be independently associated with increased fetal/infant deaths. This result was surprising as one might expect that small for gestational age infants would succumb more easily during a maternal eclamptic seizure.

\subsubsection{Infant morbidity}

The data on infant morbidity and mortality was less complete than any other type of data in the study. Therefore caution must be used in the interpretation of the results of this logistic regression analysis.

As expected from prior clinical experience and from the univariate analyses, preterm seizures were strongly associated with infant morbidity. Weight less than the tenth centile for gestational age was not independently associated with infant morbidity in this study. 
Unexpectedly the presence of symptoms in the mother was associated with an increased risk of fetal morbidity. The association was significant at the $\mathrm{P}<0.005$ level. While it is difficult to imagine a physiological basis for the association, it is not difficult to conceive of a psychological link. Mothers who experience and report symptoms themselves, may be more likely to note and report symptoms in their children. 


\section{CHAPTER 6.}

\section{CONCLUSIONS}


"I know of no previous effort to present results of a wide national scope, embracing many hospitals in different parts of the country. The results obtained clearly justify the method, although doubtless by closer co-operation and greater experience results even more valuable may be obtained in the future".

$T$ W Eden on the 1922 Report on Eclampsia

This study is the first to follow the precedent set by Eden 70 years ago and it is hoped that the results act as a foundation for future work on eclampsia.

\subsection{SUMMARY OF MAIN FINDINGS.}

Notifications of cases of eclampsia were obtained from obstetricians and midwives at all 297 hospitals with a consultant obstetric unit in the United Kingdom. Three-hundred and eighty-three cases were confirmed as eclampsia, and despite checks with existing maternity data bases, no unreported cases were discovered. It therefore appears that this study represents the total population of women with eclampsia in the United Kingdom during 1992.

Data were collected from detailed hospital case-note review and from a general practitioner questionnaire. There was a high level of complete data collection which, combined with the total ascertainment gives confidence in the accuracy of incidence rates and the maternal and fetal case fatality rates derived from the study.

The incidence of eclampsia in the United Kingdom during 1992 was 4.9/10,000 maternities (95\% confidence interval 4.5-5.4). Teenagers and women with multiple pregnancies were at particular risk of developing the condition.

In this study the great majority of cases of eclampsia occurred in women with frequent screening checks for the signs of pre-eclampsia. Ninety-seven percent of the women had some antenatal care and $71 \%$ received care which conformed to the traditional pattern. Seventeen percent of women with antenatal seizures had a screening interval between last check on blood pressure and first fit of more than 14 days.

Eclampsia occurred in a significant proportion of women without prodromal symptoms and signs indicating that even very frequent screening checks will not prevent all eclampsia. 
Thirty-three percent of the women who had been screened in the 24 hours prior to the onset of seizures did not have hypertension and proteinuria - the classical duo of pre-eclamptic signs and $7 \%$ had neither sign. Forty-one percent of all women had no antecedent symptoms.

Thirty-eight percent of fits occurred antepartum, $18 \%$ intrapartum and $44 \%$ postpartum. Twelve percent of the postpartum cases occurred more than 48 hours after delivery. Fortyfour percent of women had their first seizure preterm and over one fifth (21\%) of all fits occurred before 32 weeks completed gestation.

Prophylactic antihypertensives and anticonvulsants were used in $37 \%$ and $21 \%$ of women hospitalised prior to the onset of seizures respectively. After the onset of seizures $98 \%$ of women were given some form of anticonvulsant and $78 \%$ were given at least one antihypertensive medication. The medications most commonly used in the acute management of seizures were diazepam, phenytoin, hydralazine and labetalol.

The maternal case fatality rate from eclampsia was $1.8 \%$. Forty-five percent of women were admitted to an intensive care unit and $35 \%$ had at least one major complication associated with the eclamptic seizure. In this study there was no evidence that women over the age of 35 had any greater morbidity associated with eclampsia. Similarly, although 25\% of all cases of eclampsia occurred in multiparous women there was no evidence that they had an increased risk of morbidity from the syndrome.

The total study perinatal mortality was $7.3 \%$. There was a stillbirth rate of $22.2 / 1000$ and a neonatal mortality rate of $32.0 / 1000$. Seventy five percent of the deaths occurred in women with eclampsia before 32 completed weeks of pregnancy and $75 \%$ in infants/fetuses weighing less than 1500 grams. Twenty eight percent of the infants were small for gestational age and $56 \%$ required admission to special care baby units.

Women in this study who developed post-partum eclampsia without pre-existing sign of severe pre-eclampsia were not significantly different in terms of their outcome than all other women with eclampsia. However their infants were significantly less likely to be small for gestational age.

There appear to be real differences between antepartum eclampsia and eclampsia occurring after the onset of labour. Antepartum eclampsia appears to be associated with more severe maternal consequences. Preterm eclampsia (which often occurred antepartum) was independently associated with poorer fetal and infant outcomes. 
Multiple logistic regression analysis was carried out to assess which facets of the eclamptic illness were associated with poorer maternal and fetal outcomes. The presence of antepartum seizures, multiple seizures and high maximum systolic and/or diastolic blood pressures were all independently associated with an increased risk of developing one or more major complications. The factors associated with increased risk of general practitioner reported maternal morbidity were fetal or infant death, one or more major maternal complications, and a high first and/or maximum systolic blood pressure.

The logistic regression analysis of variables associated with fetal and infant deaths showed that preterm seizures, the lack of antenatal care and particularly preterm seizures occurring antepartum were associated with increased risk of fetal/infant death.

This is the first national study of eclampsia in over 70 years and one of very few large, population based studies of eclampsia in the developed world. It was conducted to develop an accurate picture of eclampsia in the United Kingdom in the belief that an understanding of the current situation is essential for developing sensible strategies for future improvements. The study has achieved its main aims but where does it leave us and what are the next steps in understanding the condition of eclampsia and reducing the impact of the syndrome?

\subsection{FUTURE DIRECTIONS.}

Reduction in the impact of eclampsia in the United Kingdom requires improvement in prevention, acute management and in the management of residual morbidity. In addition there needs to be continued measurement of epidemiological indices to assess accurately if real progress is being made.

Screening for pre-eclampsia/eclampsia currently relies on detecting hypertension and proteinuria and therefore cannot be expected to prevent the significant proportion of eclampsia which occurs without anticipatory signs. New, clinically applicable, screening methods are needed to identify women at risk of pre-eclampsia and eclampsia rather than just those who are already exhibiting the signs of the condition.

Current research is directed at finding the link between placental ischaemia and the endothelial cell damage which underlies the pathophysiological changes of pre-eclampsia and eclampsia (Roberts \& Redman, 1993). Once the link is understood it may be possible to develop a screening test looking for the basic pathology of the syndrome. This would potentially allow intervention prior to the onset of the signs and symptoms of the disease and may give greater chance of prevention. 
Although the ultimate aim is to prevent eclampsia realistically this will not be achieved in the immediate future and even when prevention is possible some cases will still occur. Therefore research and clinical trials should also be directed towards improving management and reducing the high rates of morbidity and mortality associated with eclampsia.

There needs to be widespread recognition by clinicians that eclampsia presents in heterogeneous manners so that when cases occur there is no delay in the initiation of management.

Every obstetric unit should have a written protocol for the management of convulsions in pregnancy to avoid delays in diagnosis and management. After a fit occurs confirmatory signs should be sought assiduously in the first 48 hours even if there are no features of preeclampsia previously. The signs should include disturbances of liver and coagulation function as well as new hypertension and proteinuria as the presence of these signs can give prior warning of maternal de-compensation.

The results of a large multi-centre randomised trial of methods for treating eclampsia conducted in South America, Asia and South Africa are due to be published shortly and are eagerly awaited (Duley, 1992b). The study should provide soundly based guidance on treatment of eclampsia. It will be important that the results of trials like these are widely disseminated and that the lessons learned from clinical trials are actually applied in the clinical setting.

The average case load per consultant obstetrician in the United Kingdom is dropping to well below 1000 deliveries a year and yet the incidence of eclampsia is approximately 1 in 2000 pregnancies. Individual doctors can expect to see the syndrome too infrequently to acquire competence in its diagnosis and management. It would therefore seem desirable that at least one consultant in every obstetric unit takes a special interest in the problem. Another approach is to have regional centres of excellence which would accept transfers of severe or early onset cases. The Confidential Enquiry into Maternal Deaths has been advocating the establishment of such centres for several years (DHSS \& Welsh Office. 1989; DHSS et al. 1991; DHSS et al. 1994) and yet few exist. Regional centres and unit specialists will not be a panacea but could provide invaluable resources of information and help in managing difficult cases.

This study has provided the first documented evidence of the minor maternal morbidity associated with eclampsia such as anxiety, headaches and depression. These types of morbidity can be major in terms of the impact they have on daily life and should not be 
underestimated. Women and their general practitioners need education about what to expect, information about future pregnancies and support in coping with their grief and fears. Regional centres of excellence may be able to provide information and to care for women in future pregnancies. Self-help, consumer based lobby groups such as APEC (Action on Pre-Eclampsia) should be supported by ensuring they have up to date, accurate and scientifically based information.

Finally there needs to be continued monitoring of the progress made in the understanding and management of eclampsia. There is a risk that because the condition is relatively rare it may drop from the consciousness of clinicians despite its high contribution to maternal mortality. It was originally hoped that at the completion of this study in 1992 a simplified eclampsia register would be established to monitor changes in the incidence and mortality from eclampsia. Unfortunately although the infrastructure for such a register existed it was not funded. It is hoped that it will not be a further 70 years before a national study of eclampsia is repeated.

The real test of progress in our knowledge of pre-eclampsia and eclampsia is whether or not we can reduce its incidence, morbidity and mortality. To properly assess progress reliable data on these indices will be needed at regular, frequent intervals not 70 years apart. 


\section{APPENDICES}

1. Data sheet for the main study.

2. General Practitioner Questionnaire.

3. Publications arising from thesis. 


\section{DATA SHEET}

1. STUDYNO (1-600)

2. REGION NHS region of hospital at which woman cared for

$1=$ East Anglia

2 = Mersey

$3=$ North East Thames

$4=$ Northern

$5=$ Northern Ireland

$6=$ North Western

$7=$ North West Thames

$8=$ Oxford

$9=$ Scotland
10. = South East Thames

11. = Southwestern

12. $=$ South West Thames

13. = Trent

14. $=$ Wales

15. $=$ Wessex

16. $=$ West Midlands

17. $=$ Yorkshire

\section{COMPLETENESS OF INFORMATION COLLECTED}

3. HOSPNOTE hospital where eclampsia occurred - notes reviewed $(\mathrm{Y} / \mathrm{N} / \mathrm{U})$

4. OTHERNOT other hospital/private practice where care given - notes reviewed (Y/N/U)

5. COOPCARD antenatal care record seen (Y/N/U)

6. GPQUEST general practitioner questionnaire completed (Y/N/U)

\section{SOCIAL AND DEMOGRAPHIC DATA}

7. DOB date of birth $(--/--/--)$

8. AGE

$$
\begin{aligned}
& 1=\leq 14 \\
& 2=15-19 \\
& 3=20-24 \\
& 4=25-29 \\
& 5=30-34 \\
& 6=35-39 \\
& 7=40-44 \\
& 8=\geq 45
\end{aligned}
$$

(record actual age and also code)

9. COUNTBTH country of birth

$$
\begin{aligned}
& 1=\text { United Kingdom } \\
& 2=\text { other European } \\
& 3=\text { Indian }
\end{aligned}
$$

$$
\begin{aligned}
& 4=\text { other Asian } \\
& 5=\text { African } \\
& 6=\text { other (specify) }
\end{aligned}
$$

10. ETHNIC ethnic origins

$$
1=\text { United Kingdom }
$$

2 = other European

$3=$ Indian

$$
\begin{aligned}
& 4=\text { other Asian } \\
& 5=\text { African } \\
& 6=\text { other (specify) }
\end{aligned}
$$

11. MARRSTAT marriage status

$$
1=\text { single }
$$

$2=$ married

$$
3=\text { widowed }
$$$$
4 \text { = divorced }
$$

12. POSTCODE

13. RESICODE residential code

14. PATOCCUP patients occupation

15. SPOUSEOCC spouses occupation

16. SOCCLASS social class according to whichever is higher rating occupation 
17. PMHHIST1 past medical history 1

18. PMHHIST2 past medical history 2

19. PMHHIST3 past medical history 3

20. PMHHIST4 past medical history 4

$1=$ no significant past medical history

2 = insulin dependent diabetes mellitus

$3=$ epilepsy

4 = renal disease (known stuctural or biopsy proven)

5 = autoimmune disorder

$6=$ hypertension requiring treatment other than in pregancy

$7=$ hypertension on oral contraceptive pill

$8=$ other (specify)

21. HVIAPREG history of viable pregnancies

$1=$ no previous viable pregnancies

2 = one previous pregnancy

$3=$ two previous pregnancies

$4=$ three previous pregnancies

$5=$ four previous pregnancies

$6=$ five previous pregnancies

$7=$ six or greater previous pregnancies

22. HNVIAPRG history of non viable pregnancies
$1=$ no previous non viable pregnancies
$2=$ TOP $<20 / 40$
$3=$ miscarriage $<20 / 40$
$4=$ TOP $<20 / 40 \times 2$
$5=$ miscarriage $<20 / 40 \times 2$
$6=$ both TOP \& miscarriage
$7=$ other (specify)

23. HISTPECL history of preeclampsia

$1=$ no previous pregnancy

$2=$ no history of preeclampsia in previous pregnancies

$3=$ history of preeclampsia in provious pregnancy

24. HISTECL history of eclampsia
$1=$ no previous pregnancy
$2=$ no history of eclampsia
3 = eclampsia in previous pregnancy

25.CIGS number of cigarrettes smoked per day at booking

$$
\begin{aligned}
& 1=\text { none } \\
& 2=\leq 5 \\
& 3=6-10
\end{aligned}
$$

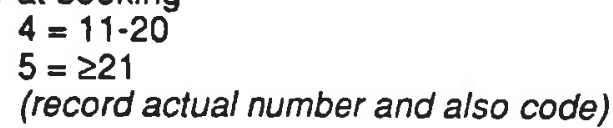

26. PREPMED1 prepregnancy medication 1

27. PREPMED2 prepregnancy medication 2

28. PREPMED3 prepregnancy medication 3

$$
\begin{aligned}
& 1=\text { none } \\
& 2=\text { anticonvulsant } \\
& 3=\text { antihypertensive }
\end{aligned}
$$

$$
\begin{aligned}
& 4=\text { steroids } \\
& 5=\text { other (specify) }
\end{aligned}
$$

\section{ANTENATAL CARE}

29. BESTEDD best estimate of expected date of confinement $(--/-/--)$

30. PLANPRE pregnancy planned (Y/N)

31. BOOKDATE date at first booking (--/--/--)

32. BOOKGEST gestation (in weeks) at first booking visit 
33. BOOKHGT booking height (in $\mathrm{cm}$ )

34. BOOKWGHT booking weight (in $\mathrm{kgs}$ )

35. BLOODGRP maternal blood group

36. FETALNO number of fetus's

$$
\begin{aligned}
& 1=\text { one } \\
& 2=\text { two } \\
& 3=\text { three }
\end{aligned}
$$

$4=$ four

5 = hydatidiform mole

37. ANCPLAN antenatal care plan

$1=$ none

$2=\mathrm{gp} /$ community midwife only

$3=$ shared gp/consultant/midwife

$4=$ consultant only

$5=$ other (specify)

38. INPLCDEL intended place of delivery

$$
\begin{aligned}
& 1=\text { none } \\
& 2=\text { gp obstetric unit } \\
& 3=\text { consultant obstetric unit }
\end{aligned}
$$

$$
\begin{aligned}
& 4=\text { home } \\
& 5=\text { other (specify) }
\end{aligned}
$$

39. ANVGPB20 number of antenatal visits by $\mathrm{gp} / \mathrm{midwife}$ before 20 weeks gestation

$$
\begin{aligned}
& 1=\text { none } \\
& 2=1 \text { visit before } 20 \text { weeks } \\
& 3=2 \text { visits before } 20 \text { weeks } \\
& 4=3 \text { visits before } 20 \text { weeks } \\
& 5=4 \text { visits before } 20 \text { weeks } \\
& 6=5 \text { visits before } 20 \text { weeks }
\end{aligned}
$$

$$
\begin{aligned}
7 & =6 \text { visits before } 20 \text { weeks } \\
8 & =7 \text { visits before } 20 \text { weeks } \\
9 & =8 \text { visits before } 20 \text { weeks } \\
10 & =9 \text { visits before } 20 \text { weeks } \\
11 & =10 \text { or more visits before } 20 \text { weeks }
\end{aligned}
$$

40. ANVCNB20 number of antenatal visits to consultant unit before 20 weeks gestation
$1=$ none
$2=1$ visit before 20 weeks
$3=2$ visits before 20 weeks
$4=3$ visits before 20 weeks
$5=4$ visits before 20 weeks
$6=5$ visits before 20 weeks

$$
\begin{aligned}
7 & =6 \text { visits before } 20 \text { weeks } \\
8 & =7 \text { visits before } 20 \text { weeks } \\
9 & =8 \text { visits before } 20 \text { weeks } \\
10 & =9 \text { visits before } 20 \text { weeks } \\
11 & =10 \text { or more visits before } 20 \text { weeks }
\end{aligned}
$$

41. ANVGPA20 number of antenatal visits to gp/midwife after 20 weeks gestation
1 = none
$7=6$ visits before 20 weeks
$2=1$ visit before 20 weeks
$3=2$ visits before 20 weeks
$4=3$ visits before 20 weeks
$5=4$ visits before 20 weeks
$6=5$ visits before 20 weeks
$8=7$ visits before 20 weeks
$9=8$ visits before 20 weeks
$10=9$ visits before 20 weeks
$11=10$ or more visits before 20 weeks

42. ANVCNA20 number of antenatal visits to consultant unit after 20 weeks gestation
$1=$ none
$2=1$ visit before 20 weeks
$3=2$ visits before 20 weeks
$4=3$ visits before 20 weeks
$5=4$ visits before 20 weeks
$6=5$ visits before 20 weeks

$$
\begin{aligned}
7 & =6 \text { visits before } 20 \text { weeks } \\
8 & =7 \text { visits before } 20 \text { weeks } \\
9 & =8 \text { visits before } 20 \text { weeks } \\
10 & =9 \text { visits before } 20 \text { weeks } \\
11 & =10 \text { or more visits before } 20 \text { weeks }
\end{aligned}
$$

43. TRCAREBF transfer of care before fit (Y/N)

44. TRCAREAF transfer of care after fit (Y/N)

45. DNAANC did not attend a booked antenatal visit $(\mathrm{Y} / \mathrm{N})$

46. DNAANADM did not accept an advised antenatal/postnatal admission prior to eclampsia (Y/N)

47. SELFDISC discharged self against advice during an antenatal/postnatal admission prior to eclampsia $(\mathrm{Y} / \mathrm{N})$ 
48. WGHTGAIN weight gain antenatally

1 = weight gain $<1 \mathrm{~kg}$ per week (averaged over time)

2 = weight gain $\geq 1 \mathrm{~kg}$ per week (averaged over time)

49. FIRSTBPS systolic BP at first antenatal visit

50. FIRSTBPD diastolic BP at first antenatal visit

51. MAXBPBFS maximum systolic BP before eclamptic seizure

52. MAXBPBFD maximum diastolic BP before eclamptic seizure

53. LASTBPBS last systolic BP before eclamptic seizure

54. LASTBBPD last diastolic BP before eclamptic seizure

55. DATELBPB date of last BP recorded before seizure (--1--/--)

56. TIMELBPB time of last BP recorded before seizure

57. INTERBPB interval between last recorded BP and seizure
$1=<1$ hour
$2=1-6$ hours
$3=7-12$ hours
$4=13-24$ hours
$5=2-3$ days
$6=4-5$ days
$7=6-7$ days
$8=8-14$ days
$9=>14$
(record actual interval and then code)

58. FSTBPAFS first recorded systolic BP after seizure

59. FSTBPAFD first recorded diastolic BP after seizure

60. DATEFBPA date of first BP recording after seizure $(--/-1--)$

61. TIMEFBPA time of first BP recording after seizure

62. INTERBPA interval between seizure and first recorded BP after seizure

$$
\begin{aligned}
& 1=<1 \text { hour } \\
& 2=1-6 \text { hours } \\
& 3=7-12 \text { hours } \\
& 4=13-24 \text { hours } \\
& 5=2-3 \text { days }
\end{aligned}
$$

$$
\begin{aligned}
& 6=4-5 \text { days } \\
& 7=6-7 \text { days } \\
& 8=8-14 \text { days } \\
& 9=>14 \\
& \text { (record actual interval and then code) }
\end{aligned}
$$

63. MAXBPAFS maximum systolic BP after seizure

64. MAXBPAFD maximum diastolic BP after seizure

65. MAXBPS maximum systolic BP at any time

66. MAXBPD maximum diastolic BP at any time

67. GESTFHT gestation that first hypertensive reading recorded

68. FRSTPROT proteinuria recorded at first antenatal visit

$$
\begin{aligned}
& 1=\text { NAD } \\
& 2=\text { Trace } \\
& 3=+
\end{aligned}
$$

$$
\begin{aligned}
& 3=++ \\
& 5=+++ \\
& 6=2++++
\end{aligned}
$$


69. LPROTBFF last measurement of proteinuria before seizure

$1=$ NAD
$2=$ Trace
$3=+$

$3=++$

$2=+++$

$6=2++++$

70. DATELPBF date of last test for proteinuria before seizure $(--1--/--)$

71. INTPBF interval between last test for proteinuria and first fit
$1=<1$ hour
$2=1-6$ hours
$3=7-12$ hours
$4=13-24$ hours
$5=2-3$ days
$6=4-5$ days
$7=6-7$ days
$8=8-14$ days
$9=>14$
(record actual interval and then code)

72. PROTAFF first measurement of proteinuria after seizure
$1=$ NAD
$2=$ Trace
$3=++$
$3=+$
$2=+++$
$6=2++++$

73. DATEFPAF date of first test for proteinuria after seizure $(--/--/-)$

74. INTPAF interval between fit and first test for proteinuria after fit
$1=<1$ hour
$6=4-5$ days
$2=1-6$ hours
$7=6-7$ days
$3=7-12$ hours
$4=13-24$ hours
$8=8-14$ days
$9=>14$
$5=2-3$ days
(record actual interval and then code)

75. MAXPROTB maximum proteinuria recorded before seizure
$1=$ NAD
$2=$ Trace
$3=++$
$3=+$
$5=++t$
$6=2++++$

76. MAXPROTA maximum proteinuria recorded after seizure
$1=$ NAD
2 = Trace
$3=++$
$3=+$
$5=+++$
$6=\geq++++$

77. MAXPROT maximum proteinuria recorded at any time
$1=$ NAD
$3=++$
$2=$ Trace
$5=+++$
$3=+$
$6=2++++$

78. ANPROT antenatal proteinuria at any time (Y/N/U) (proteinuria means a random sample of ++ or $\geq 0.5 \mathrm{gms}$ on a 24 hour collection of urine)

79. ANHT antenatal hypertension at any time (Y/N/U) (hypertension is a booking diastolic pressure of $<90 \mathrm{~mm} \mathrm{Hg}$, a maximum diastolic of $>90 \mathrm{~mm} \mathrm{Hg}$ and a diastolic increment of $\geq 25 \mathrm{~mm} \mathrm{Hg}$ )

80. ANPROPET antenatal proteinuric PET at any time (Y/N/U)

81. DATEPET date that all criteria for proteinuric PET first met $(--/--/--)$

82. ANDIAG diagnosis which appears in casenotes
$1=$ none
2 = "hypertension" or "raised BP"
$5=$ preeclampsia
3 = "pregnancy induced hypertension"
6 = fulminating preeclampsia
$4=$ ?preeclampsia
$7=$ ?eclampsia

83. MEDDURP1 medications taken consistently during pregnancy 1

84. MEDDURP2 medications taken consistently during pregnancy 2 
85. MEDDURP3 medications taken consistently during pregnancy 3
$1=$ none
2 = anticonvulsants
$3=$ antihypertensive
5 = aspirin
$6=$ antibiotics
7 = other (specify)
$4=$ steroids

86. SYMPBFF symptoms described by patient before first seizure
$1=$ none
2 = headache
6 = headach $\theta$ \& epigastric pain
3 = visual disturbances
7 = visual disturbances \& epigastric pain
4 = epigastric pain
5 = headache \& visual disturbances
$8=$ headache, visual disturbances \& epigastric pain
$9=$ Other (specify)

87. PROPANTH prophylactic antihypertensive medication
1 = none
2 = aspirin
3 = methyl dopa
4 = nifedipine
5 = oxyprenalol

$6=$ labotalol

7 = hydrallazine

$8=$ prazosin

$9=$ diuretic

10 = other (specify)

88. PROPSED prophylactic sedative
$1=$ none
2 = diazepam
3 = chlormethiazole
$4=$ morphine
$5=$ pethidine
$6=$ general anaesthetic
7 = other (specify)

89. PROPANTC prophylactic anticonvulsant medication
1 = none
4 = barbiturate
- 2 = phenytoin
3 = magnesium sulphate
$5=$ other (specify)

90. NOPROPM number of prophylactic medications used
$1=$ none
2 = one
5 = four
$3=$ two
$6=f i v e$
$4=$ three
$7=$ six or more

91. COMPREG1 complications of pregnancy 1

92. COMPREG2 complications of pregnancy 2

93. COMPREG3 complications of pregnancy 3

$1=$ none

2 = anaemia

$3=$ vaginal infection

4 = threatened miscarriage

$5=$ antepartum haemorrhage

$6=$ placental abruption

$7=$ IUGR

$8=$ other (specify)

94. INVBFSU investigation before fit of serum urea $(Y / N)$

95. VALBFSU maximum value of serum urea before fit

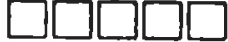

96. INVBFSC investigation before fit of serum creatinine (Y/N)

97. VALBFSC maximum value of serum creatinin before fit

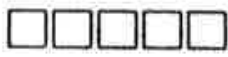

98. INVBFSB investigation before fit of serum bilinubin (Y/N)

99. VALBFSB maximum value of serum bilirubin before fit

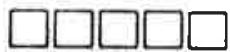

100. INVBFAST investigation before fit of AST (Y/N)

101. VALBFAST maximum value of AST before fit 
102. INVBFPLT investigation before fit platelets $(Y / N)$

103. VALBFPLT minimum value of platelets before fit

104. INVBFCLT investigation of APTT/PR before fit (Y/N)

105. VALBFCLT value of APTT/PR before fit

$1=$ normal

2 = prolonged

106. INVBFFDP investigation before fit of fibrin degredation products $(Y / N)$

107. VALBFFDP value of fibrin degredation products before fit

$1=$ normal

2 = elevated

\section{FEATURES OF ECLAMPSIA}

108. DATEFIT date of first fit $(--/--/--)$

109. TIMEFIT time of first fit

110. GESTFIT gestational age at first fit, if postpartum fit then gestational age at delivery (wks)

111. TYPEFIT type of eclampsia

$1=$ antepartum

2 = intrapartum

$3=$ postpartum

4 = unknown

112. LOCNFIT location at time of first fit

$1=$ home

$2=$ transit between home and hospital

3 = GP unit/peripheral hospital

$4=$ transit between hospitals

$5=$ consultant obstetric hospital

$6=$ other (specify)

113. NOFFITS total number of fits
$1=$ one
$5=$ five
$2=$ two
$6=\operatorname{six}$
$3=$ three
$7=$ seven or more
$4=$ four

114. NOFITSBM number of fits before management commenced
$1=$ none
2 = one
5 = four
$3=$ two
6 = five
$4=$ three
$7=\operatorname{six}$
8 = seven or more

115. NOFITSAF number of fits after management commenced
$1=$ none
5 = four
2 = one
$6=$ five
$3=$ two
$7=$ six
$4=$ three
$8=$ seven or more

116. LENHSBF length of hospital stay before first fit (days)

$\begin{array}{lrlll}1=0 & 6=4 & 11=9 & 16=14 & 21=19 \\ 2=<1 & 7=5 & 12=10 & 17=15 & 22=20 \\ 3=1 & 8=6 & 13=11 & 18=16 & 23=221 \\ 4=2 & 9=7 & 14=12 & 19=17 & \\ 5=3 & 10=8 & 15=13 & 20=18 & \end{array}$

117. MEDMAN1 medications used in management 1

118. MEDMAN2 medications used in management 2

119. MEDMAN3 medications used in management 3 
120. MEDMAN4 medications used in management 4

121. MEDMAN5 medications used in management 5

122. MEDMAN6 medications used in management 6

$$
\begin{aligned}
& 1=\text { none } \\
& 2=\text { diazepam } \\
& 3=\text { chlormethiazole } \\
& 4=\text { hydrallazine } \\
& 5=\text { nifedipine } \\
& 6=\text { methyl dopa } \\
& 7=\text { labetalol } \\
& 8=\text { prazosin } \\
& 9=\text { oxyprenalol }
\end{aligned}
$$

$1,0=$ phenytoin

$11=$ magnesuim sulphate

12 = diurectic

$13=$ morphine

$14=$ pethidin $\theta$

$15=$ general anaesthetic

$16=$ barbiturate

$17=$ other (specify)
$.1=$ IV infusion

$.2=$ IV bolus

$.3=1 \mathrm{M}$

$.4=$ oral

123. BLDTRANS blood transfusion required $(\mathrm{Y} / \mathrm{N})$

124. NOUNTBLD number of units of blood transfused

125. PLTTRANS platelets transfusion required $(\mathrm{Y} / \mathrm{N})$

126. NOUNTPLT number of units of platelets transfused

127. INVAFSU investigation after fit of serum urea $(Y / N)$

128. VALAFSU maximum value of serum urea after fit

129. INVAFSC investigation after fit of serum creatinine (Y/N)

130. VALAFSC maximum value of serum creatinine after fit

131. INVAFSB investigation after fit of serum bilirubin (Y/N)

132. VALAFSB maximum value of serum bilirubin after fit

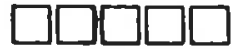

133. INVAFAST investigation after fit of AST (Y/N)

134. VALAFAST maximum value of AST after fit

135. INVAFPLT investigation after fit platelets (Y/N)

136. VALAFPLT minimum value of platelets after fit

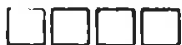

137. INVAFCLT investigation of APPT/PR after fit (Y/N)

138. VALAFCLT value of APPT/PR after fit $1=$ normal

$$
2 \text { = prolonged }
$$

139. INVAFFDP investigation after fit of fibrin degredation products (Y/N)

140. VALAFFDP value of fibrin degredation products after fit 1 = normal

2 = elevated

\section{FEATURES OF DELIVERY}

141. DATEDEL date of delivery $(--/-/--)$

142. TIMEDEL time of delivery 
144. SITEDEL site of delivery
$1=$ home
2 = transit between home \& hospital
$4=$ transit between hospitals
$3=$ GP unit/peripheral hospital
$5=$ consultant obstetric hospital

145. INTFDAF interval between fit $\&$ delivery for antenatal fits

$1=<0.5$ of an hour

$5=3: 1-4.0$ hours

$2=0.6-1$ hour

$6=4 \cdot 1-6.0$ hours

$3=1.1-2.0$ hours

$7=6.1-12$ hours

$4=2.1-3.0$ hours

$8 \Rightarrow 12$ hours (record actual interval and then code)

146. INTDFPF interval between delivery \& fit for postnatal fits

$1=<0.5$ of an hour

$2=0.6-1$ hour

$3=1.1-2.0$ hours

$5=3.1-4.0$ hours

$6=4.1-6.0$ hours

$4=2.1-3.0$ hours

$8=>12$ hours (record actual interval and then code)

147. INDUCLAB induction of labour

$1=$ no induction

$2=$ ARM

$3=$ prostaglandins

$4=$ syntocinon

$5=$ ARM \& prostaglandins

$6=$ ARM \& syntocinon

$7=$ prostaglandins \& syntocinon

$8=$ ARM \& prostaglandins \& syntocinon

148. AUGMENT augmentation of labour ( $Y / N)$

149. METHDEL method of delivery

$$
\begin{aligned}
& 1=\text { mother undelivered } \\
& 2=\text { spontaneous cephalic } \\
& 3=\text { forceps } \\
& 4=\text { ventouse } \\
& 5=\text { vaginal breech }
\end{aligned}
$$

$$
\begin{aligned}
& 5=\text { elective } \mathrm{C} / \mathrm{S} \text { before labour } \\
& 6=\text { elective } \mathrm{C} / \mathrm{S} \text { after labour } \\
& 7=\text { emergency } \mathrm{C} / \mathrm{S} \text { before labour } \\
& 8=\text { emergency } \mathrm{C} / \mathrm{S} \text { after labour }
\end{aligned}
$$

150. ANAEST type of anaesthesia used

$1=$ none

$2=\mathrm{NO} 2$

$3=$ opiates

$4=$ epidural

$5=$ general anaesthetic

$6=$ other (specifiy)

\section{MATERNAL OUTCOME}

151. COMPDEL 1 complications of delivery 1

152. COMPDEL2 complications of delivery 2.

153. COMPDEL3 complications of delivery 3

154. COMPDEL 4 complications of delivery 4
$1=$ none
2 = maternal pyrexia $\geq 38^{\circ} \mathrm{C}$
$3=$ haemorrhage $\geq 500 \mathrm{mls}$

$4=$ retained products of conception

$5=$ other (specify)

155. COMPHOS1 complications of eclampsia hospital stay 1

156. COMPHOS2 complications of eclampsia hospital stay 2

157. COMPHOS3 complications of eclampsia hospital stay 3

158. COMPHOS4 complications of eclampsia hospital stay 4 
159. COMPHOS5 complications of eclampsia hospital stay 5
$1=$ none
$8=$ cortical blindness
$2=$ urinary tract infection
9 = disemminated intravascular coagulation
$3=$ lower respiratory tract infection
$10=$ renal failure
$4=$ deep vein thrombosis
11 \& pulmonary oedema
$5=$ pulmonary embolus
$12=$ cardiac arrest
$6=$ cerebro-vascular accident
13 = required ventilation
14 = other (specify)

160. LENHSAF length of hospital stay after fit (days)

$\begin{array}{rrrrr}1=0 & 6=4 & 11=9 & 16=14 & 21=19 \\ 2=<1 & 7=5 & 12=10 & 17=15 & 22=20 \\ 3=1 & 8=6 & 13=11 & 18=16 & 23=221 \\ 4=2 & 9=7 & 14=12 & 19=17 & \\ 5=3 & 10=8 & 15=13 & 20=18 & \end{array}$

161. ADMICU admission to intensive care unit (Y/N)

162. LENICU length of stay in intensive care unit (days)

$\begin{array}{rlrl}1 & =0 & 6 & =4 \\ 2 & =<1 & 7 & =5 \\ 3 & =1 & 8 & =6 \\ 4 & =2 & 9 & =7 \\ 5 & =3 & 10 & =\geq 8\end{array}$

163. MATDEATH maternal death (Y/N)

164. TIMEDEAT timing of death

$1=$ not applicable

2 = antenatal

$3=$ intrapartum
$4=$ postpartum

165. DATEDEAT date of death $(--/-/--)$

166. CAUSEDEATH cause of death

$1=$ not applicable

2 = cerebrovascular accident

3 = pulmonary embolus

$4=$ cardiac arrest

5 = haemorrhage

$$
\begin{aligned}
& 6=\text { sepsis } \\
& 7=\text { respiratory failure } \\
& 8=\text { other (specify) } \\
& 9=\text { unknown }
\end{aligned}
$$

167. MATMORB1 maternal morbidity recorded by general practitioner 1

168. MATMORB2 maternal morbidity recorded by general practitioner 2

169. MATMORB3 maternal morbidity recorded by general practitioner 3
1 = none
2 = fever
$3=$ respiratory tract infection
$4=$ urinary tract infection
$5=$ pain in perineum
$6=$ pain in wound site
$7=$ vaginal bleeding
$8=$ hypertension
$9=$ headaches

$10=$ memory loss

$11=$ neurological deficit

12 = anxiety about baby

$13=$ anxiety about own health

$14=$ anxiety re future pregnancies

$15=$ depression

$16=$ psychosis

$17=$ other (specify)

170. REFMORB1 specialist referral required for management of morbidity 1 (Y/N)

171. REFMORB2 specialist referral required for management of morbidity 2 (Y/N) 


\section{FETAL OUTCOME}

172. FETOUT1 outcome of fetus 1

$1=$ intrauterine death

$3=$ neonatal death

2 = intrapartum death

$4=$ neonatal survivor

173. APGAR1-1 apgar at one minute for baby 1

$\begin{array}{rlrl}1 & =0 & 7 & =6 \\ 2 & =1 & 8 & =7 \\ 3 & =2 & 9 & =8 \\ 4 & =3 & 10 & =9 \\ 5 & =4 & 11 & =10 \\ 6 & =5 & & \end{array}$

174. APGAR5-1 apgar at five minutes for baby 1

$1=0$

$7=6$

$2=1$

$8=7$

$3=2$

$9=8$

$4=3$

$10=9$

$5=4$

$11=10$

$6=5$

175. SEX 1 sex of baby 1 (M/F)

176. BIRTHWGT1 birthweight of baby 1 (in grams)

177. CENTILE1 centile of birthweight baby 1
$1=<3 \%$
$2=3-10 \%$
$3=11-50 \%$
$4=51-97 \%$
$5=>97 \%$
(record actual centile and code)

178. SCBU1 time spent in SCBU baby 1 (days)
$1=$ none
$2=<1$
$3=1-7$
$4=8-14$
$5=15-21$
$6=21-28$
$7=29-56$
$B=>56$
(record actual number of days and code)

179. BLDGPB1 blood group of baby 1

180. FMORB1-1 morbidity of baby 1 number 1

181. FMORB1-2 morbidity of baby 1 number 2

182. FMORB1-3 morbidity of baby 1 number 3
$1=$ none
$2=$ respiratory problems
$3=$ developmental delay
$4=$ specific neurological deficit
$5=$ retinopathy
$6=$ other (specify)

183. CAUSBD1 cause of baby's death 1
$1=$ not applicable
$7=$ pulmonary immaturity
2 = congenital malformation
$3=$ isoimmunizatopm
8 = hyaline membrane disease
$4=$ antepartum asphyxia
9 = intracranial haemorrhage
$5=$ intrapartum asphyxia
$10=$ infection
$6=$ birth trauma
11 = miscellaneous
12 = unclassified or unknown

184. DATEDTH1 date of death of baby $1(--/ .-1--)$

185. FETOUT2 outcome of fetus 2

$1=$ intrauterine death

$3=$ neonatal death

$2=$ intrapartum death

$4=$ neonatal survivor 
186. APGAR1-2 apgar at one minute for baby 2
$1=0$
$2=1$
$3=2$
$4=3$
$7=6$
$8=7$
$9=8$
$10=9$
$5=4$
$11=10$
$6=5$

187. APGAR5-2 apgar at five minutes for baby 2

$1=0$

$2=1$

$3=2$

$4=3$

$8=7$

$9=8$

$5=4$

$10=9$

$6=5$

$11=10$

188. SEX2 sex of baby 2 (MF)

189. BIRTHWGT2 birthweight of baby 2 (in grams)

190. CENTILE2 centile of birthweight baby 2
$1=<3 \%$
$2=3-10 \%$
$4=51-97 \%$
$3=11-50 \%$
$5=>97 \%$
(record actual centile and code)

191. SCBU2 time spent in SCBU baby 2 (days)
$1=$ none
$2=<1$
$5=15-21$
$3=1-7$
$6=21-28$
$4=8-14$
$7=29-56$
$8=>56$

(record actual number of days and code)

192. BLDGPB2 blood group of baby 2

193. FMORB2-1 morbidity of baby 2 number 1

194. FMORB2-2 morbidity of baby 2 number 2

195. FMORB2-3 morbidity of baby 2 number 3

$1=$ none

$4=$ specific neurological deficit

$2=$ respiratory problems

$5=$ retinopathy

$3=$ developmental delay

$6=$ other (specify)

196. CAUSBD2 cause of baby's death 2

$1=$ not applicable $\quad 7=$ pulmonary immaturity

2 = congenital malformation $8=$ hyaline membrane disease

3 = isoimmunizatoph $\quad 9=$ intracranial haemorrhage

$4=$ antepartum asphyxia $\quad 10=$ infection

$5=$ intrapartum asphyxia $\quad 11=$ miscellaneous

6 = birth trauma $\quad 12=$ unclassified or unknown

197. DATEDTH2 date of death of baby $2(--/-/--)$

198. FETOUT3 outcome of fetus 3
$1=$ intrauterine death
$3=$ neonatal death
$2=$ intrapartum death
$4=$ neonatal survivor

199. APGAR1-3 apgar at one minute for baby 3

$\begin{array}{rlrl}1 & =0 & 7 & =6 \\ 2 & =1 & 8 & =7 \\ 3 & =2 & 9 & =8 \\ 4 & =3 & 10 & =9 \\ 5 & =4 & 11 & =10 \\ 6 & =5 & \end{array}$


200. APGAR5-3 apgar at five minutes for baby 3
$1=0$
$2=1$
$3=2$
$4=3$
$5=4$
$6=5$

$$
\begin{aligned}
7 & =6 \\
8 & =7 \\
9 & =8 \\
10 & =9
\end{aligned} .
$$$$
11=10
$$

201. SEX3 sex of baby 3 (MF)

202. BIRTHWGT3 birthweight of baby 3 (in grams)

203. CENTILE3 centile of birthweight baby 3
$1=<3 \%$
$2=3-10 \%$
$3=11-50 \%$
$4=51-97 \%$
$5=>97 \%$
(record actual centile and code)

204. SCBU3 time spent in SCBU baby 3 (days)
$1=$ none
$2=<1$
$3=1-7$
$4=8-14$
$5=15-21$
$6=21-28$
$7=29-56$
$8=>56$
(record actual number of days and code)

205. BLDGPB3 blood group of baby 3

206. FMORB3-1 morbidity of baby 3 number 1

207. FMORB3-2 morbidity of baby 3 number 2

208. FMORB3-3 morbidity of baby 3 number 3

$$
\begin{array}{ll}
1 \text { = none } & 4=\text { specific neurological deficit } \\
2 \text { = respiratory problems } & 5=\text { retinopathy } \\
3 \text { = developmental delay } & 6=\text { other (specify) }
\end{array}
$$

209. CAUSBD 3 cause of baby's death 3

$$
\begin{aligned}
& 1=\text { not applicable } \\
& 2 \text { = congenital malformation } \\
& 3=\text { isoimmunizatopm } \\
& 7 \text { = pulmonary immaturity } \\
& 4=\text { antepartum asphyxia } \\
& 5=\text { intrapartum asphyxia } \\
& 6=\text { birth trauma } \\
& 8 \text { = hyaline membrane disease } \\
& 9=\text { intracranial haemorrhage } \\
& 10 \text { = infection } \\
& 11 \text { = miscellaneous } \\
& 12 \text { = unclassified or unknown }
\end{aligned}
$$

210. DATEDTH3 date of death of baby $3(--/--/--)$

\section{MEMO}

(record field number and memo relating to field) 
212. INVBFURT investigation before fit of serum urate $(Y / N)$

213. VALBFURT maximum value of serum urate before fit

214 MEDMAN7 medications used in management 7
$1=$ none
$10=$ phenytoin
2 = diazepam
11 = magnesium sulphate
3 = chlormethiazole
4 = hydrallazine
$12=$ diuretic
$13=$ morphine
$5=$ nifedipine
$14=$ pethidine
6 = methyl dopa
$15=$ general anaesthetic
7 = labetalol
$16=$ barbiturate
8 = prazosin
9 = oxyprenalol
$17=$ other $($ specify)

215. INVAFURT investigation after fit of serum urate (Y/N)

216. VALAFURT maximum value of serum urate after fit

217. TIMEDEL2 time of delivery fetus 2

$.1=\mathrm{IV}$ infusion

$.2=$ IV bolus

$.3=\mathrm{IM}$

$.4=$ oral

.5 = sublingual

218. TIMEDEL3 time of delivery fetus 3

219. METHDEL2 method of delivery fetus 2

220. METHDEL3 method of delivery fetus 3

$1=$ mother undelivered

6 = elective $\mathrm{C} / \mathrm{S}$ before labour

2 = spontaneous cephalic

7 = elective $\mathrm{C} / \mathrm{S}$ after labour

3 = forceps

4 = ventouse

5 = vaginal breech

8 = emergency $\mathrm{C} / \mathrm{S}$ before labour

$\mathbf{9}=$ emergency $\mathrm{C} / \mathrm{S}$ after labour

221. ANAEST2 type of anaesthesia used no. 2

222. ANAEST3 type of anaesthesia used no. 3
$1=$ none
4 = epidural
$2=\mathrm{NO}_{2}$
$5=$ general anaesthetic
$3=$ opiates
$6=$ other (specify)

223. MATMORB4 maternal morbidity recorded by general practitioner 1

$1=$ none

$10=$ memory loss

2 = fever

11 = neurological deficit

$3=$ respiratory tract infection

4 = urinary tract infection

12 = anxiety about baby

5 = pain in perineum

6 = pain in wound site

$7=$ vaginal bleeding

13 = anxiety about own health

$8=$ hypertension

$14=$ anxiety re future pregnancies

$9=$ headaches

$15=$ depression

$16=$ psychosis

$17=$ other (specify)

$$
\begin{aligned}
& .1=\text { mild } \\
& .2=\text { moderate } \\
& .3=\text { severe }
\end{aligned}
$$

224. FMORB1-4 morbidity of baby 1 number 4

225. FMORB2-4 morbidity of baby 2 number 4 
226. FMORB3-4 morbidity of baby 3 number 4
$1=$ none
4 = specific neurological deficit
2 = respiratory problems
3 = developmental delay
5 = retinopathy
6 = other (specify)

227. CLASSIF classification of case
1 = classical eclampsia
2 = eclampsia but only + protein
3 = eclampsia with other reason for question of eligability
4 = fulmination preeclampsia only
5 = epilepsy
6 = other cause for seizure 


\section{ANTENATAL CARE FORM}

Blood Urine Fundal

of

Gestation Weight

Blood

Urine

Fundal

Presentation

Visit

(weeks)

Pressure

(protein)

Height

Remarks

(GP/MW/Hospital, scans, blood tests,

$(\mathrm{mm} \mathrm{Hg}$ ) (glucose)

(GP/MW/Hospital, scans
or intercurrent illnesses)

- 


\section{GENERAL PRACTITIONER QUESTIONNAIRE}

\section{PATIENT STUDY NO.}

According to our records this woman had an eclamptic seizure at on

Her L.M.P. for this pregnancy was which was certain / uncertain.

Her E.D.D. was which was revised / unrevised.

Her actual delivery date was

(Please correct any of the above if they do not correlate with your records.)

If this woman is no longer registered with you the please tick _

\section{PRE PREGNANCY:}

(please fill in these values from your records or ring not available if unknown)

- Blood pressure

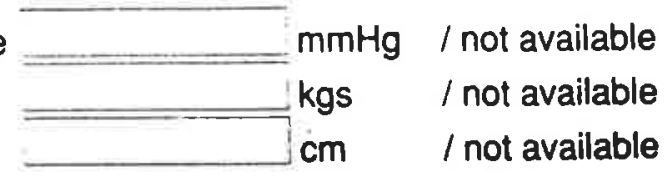

- Weight

a Height cm / not available

\section{BOOKING:}

Date of first visit to you during this pregnancy

Antenatal care was undertaken by;

(tick one only)

Consultant only

GP only

GP \& midwife

GP, other doctor \& midwite

GP, consultant \& midwile

Unknown

Other - please specify

- Plan for place of delivery;

(tick one only)

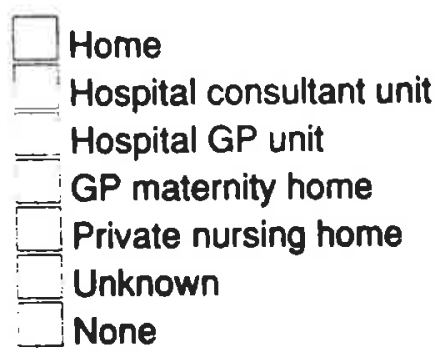

- Was booking for delivery changed?$$
\text { Yes }
$$$$
\text { Unknown }
$$
- If yes, to what changed?

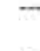 Unknown
- If yes, then why was it changed? 


\section{ANTENATAL CARE:}

BEST is trying to collect the full antenatal care record of every woman that has an eclamptic fit during 1992.

- Have you or the community midwives attached to your practice provided any antenatal care for this woman?

Yes

No

Unknown

(If Yes then please photocopy the patients co-operation card (or other antenatal care record) and include It with this questionnaire. If you do not have access to a photocopier (or do not wish to photocopy the notes) then we ask that you copy all the relevant information from ALL visits onto the blank form on the back page of this questionnaire.)

- Maximum BP recorded by you in pregnancy; BP

Date

Maximum protein on urinalysis

Protein

Date

\section{PRODROMAL SYMPTOMS AND SIGNS:}

Did this woman see you, your partners or the community midwife in the two weeks prior to

$\begin{array}{ll} & \text { Yes } \\ \text { If yes, did she complain of; } & \text { headache } \\ \text { (tick all that are applicable) } & \text { epigastric pain } \\ & \text { visual disturbance } \\ & \text { other symptoms } \\ \text { (please specify) }\end{array}$

- If yes, what was her; B.P.

Urinalysis at the time of the visit.

- Did this woman seek an extra or unscheduled appointment or ring for advice because of any of the above symptoms in the two weeks prior to

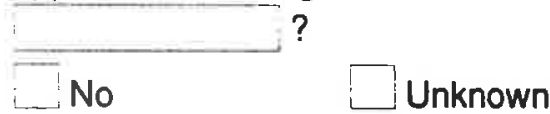

\section{POST ECLAMPSIA:}

Yes

No

Unknown

- Have you seen this woman since her eclamptic fit on

Yes

No

Unknown

(If Yes, please fill in the following chart by ticking the box next to any symptom or sign that was discussed or that you felt was an issue during the consultation. Please record the date of the consultation and also grade the severity of the problem)

\begin{tabular}{lll}
$\begin{array}{l}\text { Main Reason for } \\
\text { Consultation }\end{array}$ & $\begin{array}{l}\text { Date of } \\
\text { Consultation }\end{array}$ & $\begin{array}{l}\text { Severity of Problem; } \\
\text { Mild/Moderate/Severe }\end{array}$ \\
\hline Fever & & $\begin{array}{l}\text { Mild/Moderate/Severe } \\
\text { Mespiratory tract infection }\end{array}$ \\
Mrinary tract infection & Mild/Moderate/Severe \\
Pain in perineum & Mild/Moderate/Severe \\
Pain in wound site & Mild/Moderate/Severe \\
Vaginal bleeding & Mild/Moderate/Severe \\
Hypertension & Mild/Moderate/Severe \\
Headaches & Mild/Moderate/Severe \\
Memory loss & Mild/Moderate/Severe \\
Neurological deficit & Mild/Moderate/Severe \\
Anxiety about baby & Mild/Moderate/Severe \\
Anxiety about own health & Mild/Moderate/Severe \\
Anxiety re future pregnancies & Mild/Moderate/Severe
\end{tabular}




\begin{tabular}{lll}
$\begin{array}{l}\text { Main Reason for } \\
\text { Consultation }\end{array}$ & $\begin{array}{l}\text { Date of } \\
\text { Consultation }\end{array}$ & $\begin{array}{l}\text { Severity of Problem; } \\
\text { Mild/Moderate/Severe }\end{array}$ \\
\hline Depression & & $\begin{array}{l}\text { Mild/Moderate/Severe } \\
\text { Mild/Moderate/Severe } \\
\text { Psychosis }\end{array}$ \\
Other & & $\begin{array}{l}\text { Mild/Moderate/Severe } \\
\text { (please specify) }\end{array}$ \\
& Mild/Moderate/Severe \\
Mild/Moderate/Severe
\end{tabular}

- Has this woman required specialist referral or hospital admission for any of these symptoms?
Yes
No
Unknown

If yes then for which ones?

\section{FETAL OUTCOME}

Because of the anonomisation of records sent to us, BEST has extreme difficulty in tracing the fetal outcomes of pregnancies complicated by eclampsia. If at all possible please help us by filling out the following section referring to fetal outcome.

- This infant was

$$
\begin{aligned}
& \square \text { Liveborn and is surviving } \\
& \square \text { Liveborn but died in neonatal period } \\
& \text { Stillborn } \\
& \text { Unknown }
\end{aligned}
$$

- Sex of infant

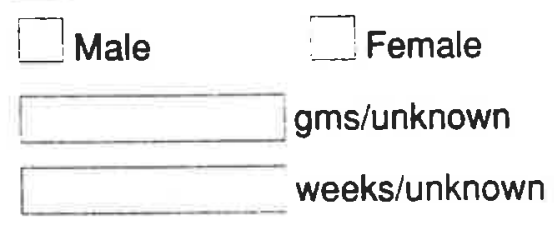

- Gestational age

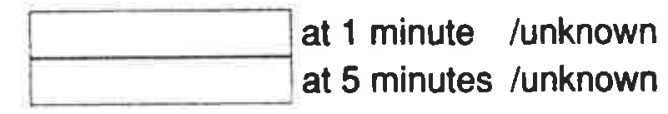

- If liveborn then the apgars at birth at 5 minutes /unknown

- If liveborn then did the infant suffer with any significant morbidity.
[. Yes
$\square$ No
Unknown

If yes please describe the type of morbidity:

If yes then are these problems ongoing?
$\square$ Yes
No

For neonatal deaths; what was the cause of death? date of death

For stillborn infants; what was the cause of death?

THANK YOU FOR COMPLETING THIS QUESTIONNAIRE

(Please check that you have completed all the questions and that you have either, filled in the blank antenatal care form over-leaf or, have included a photocopy of the woman's antenatal record)

\section{PLEASE RETURN TO BEST IN THE ENCLOSED POSTAGE PAID ENVELOPE AS SOON AS POSSIBLE - THANKS AGAIN}


Date of

Visit

Gestation Weight

(weeks)

(kgs)

Blood

(protein)

Height

( $\mathrm{mm} \mathrm{Hg}$ ) (glucose)
Presentation

Remarks

GP/MW/Hospital, scans,blood tests,

or intercurrent illnesses) 
Douglas, K. A., \& Redman, C. W. G. (1992). Eclampsia in the United Kingdom. The 'BEST' way forward. Btitish Journal of Obstetrics \& Gynaecology, 99(5), 355-356.

NOTE:

This publication is included in the print copy of the thesis held in the University of Adelaide Library.

It is also available online to authorised users at: https://doi.org/10.1111/j.1471-0528.1992.tb13745.x 


\title{
Eclampsia in the United Kingdom
}

\author{
K A Douglas, C W G Redman
}

\begin{abstract}
Objectives-To measure the incidence of eclampsia, establish how often it is preceded by signs of pre-eclampsia, document the morbidity associated with eclampsia, and determine the maternal case fatality rates.

Design-A prospective, descriptive study of every case of eclampsia in the United Kingdom in 1992. Information was collected from reviews of hospital case notes and questionnaires to general practi-
\end{abstract} tioners.

Setting-All 279 hospitals in the United Kingdom with a consultant obstetric unit.

Results-Obstetricians and midwives notified 582 possible cases, and 383 were confirmed as eclampsia. The national incidence of eclampsia was $4 \cdot 9 / 10000$ maternities ( $95 \%$ confidence interval 4.5 to 5.4 ). Most convulsions occurred despite antenatal care $(70 \%)$ and within one week of the woman's last visit to a doctor or midwife (85\%). Three quarters of first seizures occurred in hospital, of which $38 \%$ developed before both proteinuria and hypertension had been documented. Forty four per cent of cases occurred postpartum, more than a third (38\%) antepartum, and the remainder (18\%) intrapartum. Nearly one in 50 women $(1.8 \%)$ died, and $35 \%$ of all women had at least one major complication. The rate of stillbirths and neonatal deaths was $22 \cdot 2 / 1000$ and $34 \cdot 1 / 1000$, respectively. Preterm eclampsia occurred more commonly antepartum and was associated with more maternal complications and fetuses that were small for gestational age, as well as with higher rates of stillbirth and neonatal mortality. Antepartum eclampsia, which was more likely to occur preterm, was associated with a higher rate of maternal complications and a higher neonatal mortality. Both factors (gestational prematurity and antepartum occurrence) contributed independently to the severity of the outcome.

Conclusion-Eclampsia occurs in nearly one in 2000 maternities in the United Kingdom and is associated with high maternal morbidity and fatality in cases. It may present unheralded by warning signs. Preterm and antenatal eclampsia seem to be particularly severe.

Nuffield Department of

Obstetrics and Gynaecology, John Radcliffe Hospital, Oxford University, Oxford OX3 9DU

KA Douglas, visiting research fellow

CW G Redman, professor of obstetric medicine

Correspondence to: Professor Redman.

\section{Introduction}

Eclampsia is the occurrence of convulsions in association with the signs and symptoms of pre-eclampsia. The syndrome of pre-eclampsia can affect all maternal organ systems, but it is usually detected by the presence of new hypertension, proteinuria, and oedema in pregnancy. ${ }^{\text {Th }}$ Those involved in obstetric care in the United Kingdom deal with pre-eclampsia daily but may perceive eclampsia as a problem of the past now limited to developing countries. In fact eclampsia is still a major cause of maternal mortality in the United Kingdom and has been since the 1950 s. $^{2}$

There are no systematically collected population based data that allow the incidence of eclampsia or the morbidity and mortality associated with it to be measured. The only previous national study of eclampsia was conducted in $1922,{ }^{3}$ before the advent of routine antenatal screening. The British eclampsia survey was conducted in 1992 to redress this dearth of information. The aims were to measure the incidence of eclampsia and its maternal and perinatal mortality. ${ }^{4}$

\section{Methods}

A protocol detailing the aims and methodology of the study was sent to every local research ethics committee in the United Kingdom, and in all cases when formal approval was deemed to be necessary it was sought and obtained. The Royal College of Obstetricians and Gynaecologists, the Royal College of Midwives, and the Royal College of General Practitioners all formally approved the protocol and encouraged their members to participate.

Requests for notifications of any case of possible eclampsia or unexplained seizure occurring antenatally, intrapartum, or in the first 10 days postpartum during 1992 were sent every three months to all 1011 consultant obstetricians in the United Kingdom and a midwife contact at each of the 279 hospitals with an obstetric unit. A reply was requested whether or not there was a case to be reported, and reminders were sent to non-responders six weeks later. After the notification a photocopy of the woman's entire hospital case notes was sent to us after identifying data had been removed. A detailed review of the case notes was then completed by one of us (KAD), and cases were included if they met the criteria outlined below.

Eclampsia was defined as the occurrence of convulsions during pregnancy or in the first 10 days postpartum together with at least two of the following features within 24 hours after the convulsions: hypertension (a booking diastolic pressure of $<90 \mathrm{~mm} \mathrm{Hg}$, a maximum diastolic of $\geqslant 90 \mathrm{~mm} \mathrm{Hg}$, and a diastolic increment of $\geqslant 25 \mathrm{~mm} \mathrm{Hg}^{5}$ ); proteinuria (at least + protein in a random urine sample or $\geqslant 0.3 \mathrm{~g}$ in a 24 hour collection); thrombocytopenia (platelet count of less than $\left.100 \times 10^{\circ} / 1\right)$; or an increased plasma aspartate transaminase concentration ( $\geqslant 42 \mathrm{IU} / \mathrm{l})$.

Data were collected on antepartum and intrapartum care, the eclamptic episode, and maternal and perinatal outcomes. Antenatal care was assessed relative to a typical pattern of a booking visit before 20 weeks' gestation, monthly visits until 30 weeks, fortnightly visits until 36 weeks, and weekly visits thereafter. Care that did not conform to this pattern did not necessarily imply substandard care.

The gestational age at the time of seizure was recorded, and for postpartum seizures this was taken to be the gestational age at delivery. Eclampsia was defined as unheralded if the woman was in hospital and did not have established proteinuria and hypertension before the fit, although these signs then had to be documented in the first 24 hours afterwards. Maternal 
complications were defined as those recorded in hospital case notes and were classified as major (for example, disseminated intravascular coagulopathy, renal failure, cerebrovascular accident) or minor (for example, infection of urinary tract or lower respiratory tract).

General practitioners were sent a questionnaire about antenatal care, maternal puerperal morbidity, and neonatal outcome. Posteclamptic morbidity was assessed by general practitioners reporting if they had seen the woman and if any of 16 listed factors had been an issue during consultation.

Denominator data for live births and stillbirths in the United Kingdom were obtained from the Office of Population Censuses and Surveys for England and Wales, the report of the general registrar of Scotland, and from the office of the general registrar for Northern Ireland. A maternity denotes a pregnancy which resulted in a stillbirth or live birth. A stillbirth refers to a child born after the 24th week of pregnancy without any sign of life. Fetuses were classified as small for gestational age if their birth weights were less than the 10th centile for their gestational age by using charts based on Oxford data. ${ }^{6}$

Means (SD) are given for continuous variables. Groups of continuous variables were compared by using the Kruskal-Wallis test for two groups. Confidence intervals for rates were calculated. Further statistical analyses utilised $\chi^{2}$ test with Yates's correction, Fisher's exact test, and $\chi^{2}$ test for trend when appropriate (with $1 \mathrm{df}$ unless otherwise stated). Relative risks and the associated 95\% confidence intervals were also calculated. For stratified analyses the Mantel-Haenszel summary $\chi^{2}$ and summary relative risks were calculated.

\section{Results}

Participation-All 279 (100\%) obstetric units responded to at least three of our four requests for information. Ninety five per cent (265) of hospitals responded to all four requests, and there were no significant differences in the response rates from individual countries.

Outcome of notifications-Nine hundred and twenty forms with notifications were received detailing 582 individual cases. Figure 1 shows the outcome of the 582 notifications. Sixty cases reported by midwives were deemed inappropriate by consultants, but otherwise notifications from both sources agreed. Three hundred and eighty three cases were included as eclampsia, and in $49 \%$ (189) of these we had received more than one notification of the case. The lower part of figure 1 shows the completeness of data collection.

Ascertainment-Confirmed cases were compared with those registered in the Cardiff births survey, in the North West Thames regional database, in the Scottish Information and Statistics Division maternity data, and with the Department of Health's register of maternal deaths. In each comparison we have recorded a greater number of cases than the existing database system.

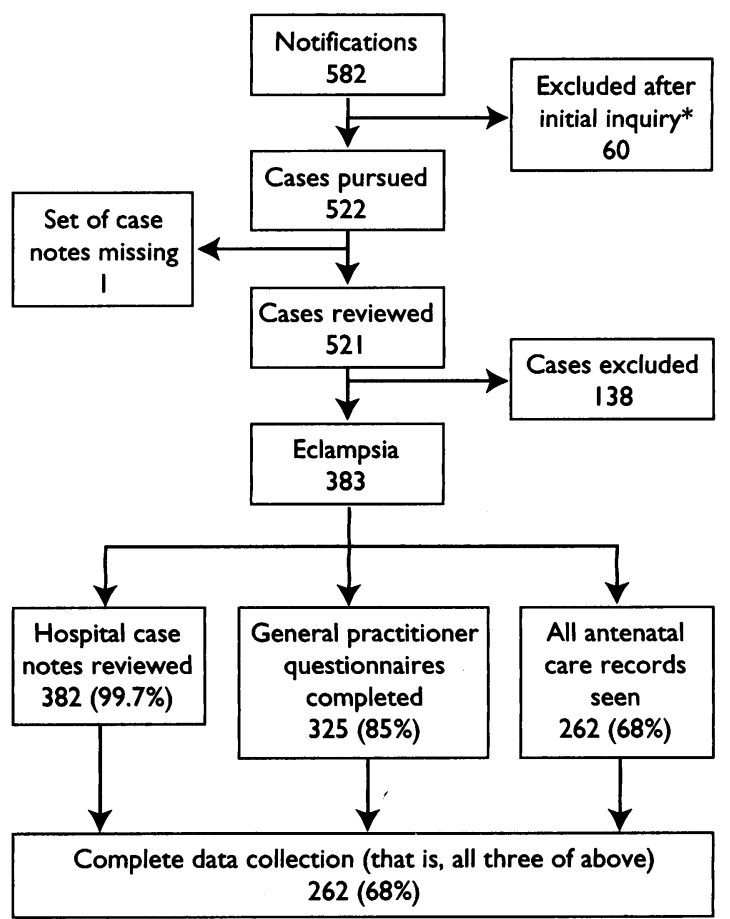

* Consultants concerned thought notification inappropriate either because no convulsion had occurred or because it was fully explained by diagnosis other than eclampsia

FIG 1-Outcome of notifications and completeness of data collection

Incidence-There were 774436 maternities in the United Kingdom during 1992 and 383 confirmed cases of eclampsia, which gives an incidence of $4.9 / 10000$ maternities (95\% confidence interval 4.5 to 5.4 ) (table I). The incidence in the individual regional health authorities of England varied from 3.4/10000 to $7 \cdot 6 / 10000$, but the differences were not significant.

Risk factors-Teenagers were three times more likely to suffer eclampsia than were older women (relative risk ( $95 \%$ confidence interval) $3.0(2 \cdot 4$ to 4.0$)$ ) (table I). Women with multiple pregnancies were also significantly more susceptible (table I), with a relative risk of $6.0(4 \cdot 1$ to $8 \cdot 9)$. No increase was detected in women over the age of 34 years. Twenty six (27\%) of the 96 women with previous viable pregnancies had a history of pre-eclampsia, and one had a history of eclampsia. Thus $18 \%$ (70) of all cases were parous women with no previous history of pre-eclampsia or eclampsia.

Antenatal care-Thirteen women (3\%) had no antenatal care before the onset of convulsions. Of the remainder it was possible to review the entire record of antenatal visits in $291(79 \%)$ cases. Of these, $206(71 \%)$ had antenatal care corresponding to or exceeding our standard pattern. Eighteen women $(6 \%)$ booked after 20 weeks, and $67(23 \%)$ had less than the standard frequency of visits. Women with less frequent antenatal visits were not significantly different from those with standard antenatal care in terms of the type of first seizure, where it occurred, or the gestational age at

TABLE I-Incidence rates of eclampsia in United Kingdom by country, maternal age, and number of fetuses

\begin{tabular}{|c|c|c|c|c|c|c|c|c|c|c|}
\hline \multirow[b]{2}{*}{ Detail } & \multirow[b]{2}{*}{$\begin{array}{c}\text { United } \\
\text { Kingdom }\end{array}$} & \multicolumn{4}{|c|}{ Country } & \multicolumn{3}{|c|}{ Maternal age (years) } & \multicolumn{2}{|c|}{ Number of fetuses } \\
\hline & & England & Scotland & Wales & $\begin{array}{l}\text { Northern } \\
\text { Ireland }\end{array}$ & $\leqslant 19$ & $20-34$ & $\geqslant 35$ & Multiple & Singleton \\
\hline $\begin{array}{l}\text { No of cases of eclampsia } \\
\text { No of maternitiest } \\
\text { Rate of eclampsia/10 } 1000 \text { maternities ( } 95 \% \text { confidence } \\
\text { interval) }\end{array}$ & $\begin{array}{c}383 \\
774436 \\
4 \cdot 9 \\
(4 \cdot 5 \text { to } 5 \cdot 4)\end{array}$ & $\begin{array}{c}307 \\
646456 \\
4 \cdot 7 \\
(4 \cdot 2 \text { to } 5 \cdot 3)\end{array}$ & $\begin{array}{c}44 \\
65307 \\
6 \cdot 7 \\
(4 \cdot 6 \text { to } 8 \cdot 7)\end{array}$ & $\begin{array}{c}15 \\
37248 \\
4 \cdot 0 \\
(2 \cdot 0 \text { to } 6 \cdot 1)\end{array}$ & $\begin{array}{c}17 \\
25425 \\
6 \cdot 7 \\
(3 \cdot 5 \text { to } 9 \cdot 9)\end{array}$ & $\begin{array}{c}72 \\
54885 \\
13 \cdot 1 \ddagger \\
(10 \cdot 1 \text { to } 16 \cdot 1)\end{array}$ & $\begin{array}{c}278 \\
645246 \\
4 \cdot 3 \\
(3 \cdot 8 \text { to } 4 \cdot 8)\end{array}$ & $\begin{array}{c}33 \\
74244 \\
4 \cdot 4 \\
(2 \cdot 9 \text { to } 6 \cdot 0)\end{array}$ & $\begin{array}{c}27 \\
9618 \\
28 \cdot 1 \oint \\
(17 \cdot 5 \text { to } 38 \cdot 6)\end{array}$ & $\begin{array}{c}356 \\
764818 \\
4 \cdot 7 \\
(4 \cdot 2 \text { to } 5 \cdot 1)\end{array}$ \\
\hline
\end{tabular}

*OPCS population data; maternal age unknown in 61 cases.

*

$\dagger$ Population data on number of maternities from OPCS VS2 1992, general registrar's office Northern Ireland, and general registrar's office Scotland

$\delta X^{2}$ with Yates's correction $=100 \cdot 4, P<0.0001$; relative risk $(95 \%$ confidence interval) $6.0(4 \cdot 1$ to $8 \cdot 9)$ of women with multiple pregnancies compared with those with singleton pregnancies. 
which it occurred. Nor were any differences detected in terms of maternal or fetal outcome (data not shown).

Premonitory signs and symptoms-Three hundred and twenty five $(85 \%)$ women had been seen by a doctor or midwife in the week before their first convulsion. At the time of the last antenatal visit $36(11 \%)$ had no recorded hypertension or proteinuria, $32(10 \%)$ had proteinuria but no hypertension, $71(22 \%)$ had hypertension alone, and $186(57 \%)$ women had both proteinuria and hypertension. Two hundred and ninety four $(77 \%)$ women were in hospital when eclampsia first occurred, and even in this subgroup only $182(62 \%)$ had established proteinuria and hypertension before the first fit. Sixty four women for whom we had a complete record of antenatal care had their first convulsion while under community based care, and five $(8 \%)$ of these women had documented proteinuria and hypertension before their first convulsion. Two hundred and twenty seven (59\%) women had one or more antecedent symptoms including headaches in 188 $(50 \%)$, visual disturbances in $72(19 \%)$, and epigastric pain in $71(19 \%)$. Fourteen women $(4 \%)$ had all three symptoms before convulsing. The maximum blood pressures before the onset of eclampsia were often not dramatically increased: of those who were in hospital the highest recorded diastolic blood pressure before the onset of seizures was $100 \mathrm{~mm} \mathrm{Hg}$ or less in 100 $(34 \%)$. In 201 women the blood pressure was measured within one hour of the onset of seizures, and the mean (SD) diastolic blood pressure was $97(14 \cdot 6) \mathrm{mm} \mathrm{Hg}$. Very high diastolic blood pressures $(\geqslant 120 \mathrm{~mm} \mathrm{Hg}$ ) before the onset of convulsions were recorded in only $19 \%(70)$ of women overall $(23 \%(69)$ in women receiving hospital care and $2 \%$ (one) in women under community care).

Timing of fit in relation to labour-One hundred and forty seven women (38\%) had antepartum eclampsia, 68 women (18\%) intrapartum, and $168(44 \%)$ postpartum eclampsia. Twenty $(12 \%)$ of the postpartum cases occurred more than 48 hours after delivery, three $(2 \%)$ more than seven days after delivery.

Investigations-Investigations before the onset of eclampsia were not routinely done; in women admitted to hospital with established proteinuria and hypertension only $54 \%(98 / 182)$ had plasma creatinine concentrations measured and $30 \%(54 / 182)$ had plasma transaminase concentrations measured. Even after the onset of eclampsia only $63 \%$ (240) of the women had their plasma transaminase concentrations measured.

Maternal outcome-There were seven maternal deaths, giving a maternal case fatality of $1.8 \%(95 \%$ confidence interval $0 \cdot 7 \%$ to $3 \cdot 7 \%$ ). In addition to the maternal deaths one woman was left in a persistent vegetative state after a massive cerebrovascular haemorrhage. One hundred and thirty five women $(35 \%)$ had at least one major complication (table II) The length of hospital stay after eclampsia varied from

TABLE II-Major maternal complications in eclampsia recorded in hospital case notes

\begin{tabular}{lcc}
\hline Major maternal complications & No of cases $(\mathrm{n}=382)^{\star}$ & $\%$ Of cases \\
\hline Death & 7 & $1 \cdot 8$ \\
Persistent vegetative state & 1 & $<1$ \\
Cardiac arrest & $6(4)$ & $1 \cdot 6$ \\
Cerebrovascular accident & $7(1)$ & $1 \cdot 8$ \\
Adult respiratory distress syndrome & $7(3)$ & $1 \cdot 8$ \\
HELLP† & $27(2)$ & 7 \\
Disseminated intravascular coagulopathy (other than HELLP) & $33(3)$ & 5 \\
Pulmonary oedema & 18 & $1 \cdot 0$ \\
Mendleson's syndrome & $4(1)$ & 6 \\
Renal failure & $24(4)$ & $1 \cdot 3$ \\
Pulmonary embolus & 5 & $<1$ \\
Septicaemia & $2(1)$ & $<1$ \\
Cortical blindness (transient) & 2 & 23 \\
Required ventilation & $87(6)$ & \\
\hline
\end{tabular}

*Figures in parentheses are complications suffered in the seven fatal cases of eclampsia.

tHELLP- Haemolysis, elevated liver enzymes, and low platelets syndrome. two days to more than 40 days; $46(12 \%)$ women stayed more than two weeks and $19(5 \%)$ for more than three weeks. One hundred and ninety four (63\%) of the 306 women whose general practitioners responded had some documented posteclamptic morbidity. Hypertension was the most common problem $(23 \%(69))$, but anxieties about personal health $(14 \%(44))$, future pregnancies $(14 \%(44))$, headaches $(12 \%(37))$, and depression $(10 \%$ (29)) were also common.

Fetal and infant outcome-There were 411 fetuses; 356 singletons, 26 twins, and one set of triplets. All but one of the fetuses were weighed at delivery: $35(9 \%)$ weighed $1000 \mathrm{~g}$ or less and $44(11 \%)$ weighed between 1001 and $1500 \mathrm{~g}$. Of the 356 singletons, $100(28 \%)$ were small for gestational age. Thirty fetal and infant deaths were reported. Five of these were intrauterine deaths in women who were delivered between 20 and 24 weeks' gestation because of worsening maternal condition. There were nine stillbirths in fetuses over 24 weeks' gestation (stillbirth rate $22 \cdot 2 / 1000 ; 95 \%$ confidence interval $10 \cdot 2$ to $41 \cdot 7$ ), and 13 infants died in the neonatal period (neonatal mortality $32.0 / 1000(17 \cdot 2$ to $54 \cdot 1)$ ). In addition, three deaths were reported from complications of prematurity in infants aged more than 4 weeks. All of the deaths were among singletons, most $(80 \%$ (24)) weighing less than $1500 \mathrm{~g}$ at delivery.

\section{FURTHER ANALYSES}

It has been previously suggested that preterm eclampsia has more serious maternal and fetal consequences than eclampsia at term ${ }^{7}$ and that eclampsia is less severe when it develops postpartum. ${ }^{8}$ To test these hypotheses we analysed in more detail the type of convulsion (antepartum, intrapartum, or postpartum) and the gestational age at which the crisis occurred.

Type of eclampsia and gestational maturity- Almost half $(44 \%$ (169)) of the cases presented preterm (before 37 completed weeks of gestation), and over a fifth ( $21 \%$ (81)) developed before 31 weeks. The type of eclampsia was closely associated with gestational maturity (fig 2 ).

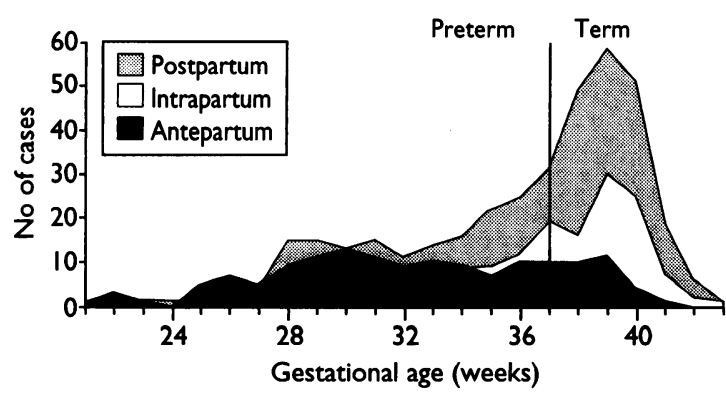

FIG 2-Number of cases of eclampsia by gestational age at first seizure. For postpartum convulsions gestational age at first seizure taken as gestational age at delivery

Most of the antepartum convulsions (76\%; 111/147) occurred preterm; most of those that were intrapartum or postpartum $(75 \% ; 178 / 236)$ presented at term. We compared antepartum with intrapartum and postpartum eclampsia. Antepartum eclampsia was more often associated with prodromal symptoms (relative risk $1.23(1.04$ to 1.47$)$ ) and less often with prodromal signs (relative risk $0.87(0.78$ to 0.96$))$. Antepartum cases were in some respects more severe than those occurring at or after delivery: there were significantly more cases with multiple convulsions, major maternal complications, small for gestational age fetuses, and a significantly higher neonatal mortality. Table III summarises the details. Preterm and term cases were also compared. Maternal age, parity, and the number of antenatal visits were similar in the preterm and term groups (data not shown). Women with preterm eclampsia were more severely affected than those presenting at or after term (table IV). Given the close 


\begin{tabular}{|c|c|c|c|c|}
\hline Factor of interest & $\begin{array}{l}\text { No }(\%) \text { of } \\
\text { antepartum cases } \\
(n=147)\end{array}$ & $\begin{array}{c}\text { No (\%) of } \\
\text { intrapartum and } \\
\text { postpartum cases } \\
(n=236)\end{array}$ & $\begin{array}{l}\text { Relative risk }{ }^{\star} \\
(95 \% \text { confidence interval) }\end{array}$ & $\begin{array}{c}\begin{array}{c}\text { Summary relative risk } \\
\text { with stratified analysis }\end{array} \\
\text { (corrected for gestation } \\
\text { of fit) } \\
\text { (95\% confidence interval) }\end{array}$ \\
\hline Prodromal symptoms & $107(75)$ & $120(51)$ & $1.48(1.26$ to 1.73$)$ & $1.23(1.04$ to 1.47$)$ \\
\hline Prodromal signs ( 325 seen within past seven days) & $76(83)$ & $213(91)$ & $0.90(0.82$ to 1.00$)$ & $0.87(0.78$ to 0.96$)$ \\
\hline Preterm seizures & $111(76)$ & $58(25)$ & $3.07(2.41$ to 3.91$)$ & NA \\
\hline Seizure outside hospital & $82(56)$ & $6(3)$ & 22.09 (9.90 to 49.30$)$ & $36.24(9.43$ to $139 \cdot 36)$ \\
\hline Multiple seizures & $78(54)$ & $80(34)$ & $1.59(1.26$ to 2.00$)$ & $1.52(1.15$ to 1.99$)$ \\
\hline Delivered by caesarean section & $135(92)$ & $71(30)$ & $3.05(2.50$ to 3.73$)$ & $2.29(1.90$ to 2.76$)$ \\
\hline Major maternal complication & $74(51)$ & $61(26)$ & $1.96(1.50$ to 2.57$)$ & $1.63(1.21$ to 2.19$)$ \\
\hline Maternal death & $3(2)$ & $4(2)$ & $1.20(0.27$ to 5.30$)$ & NA \\
\hline Small for gestational age & $62(44)$ & $38(18)$ & $2.42(1.72$ to 3.42$)$ & $6.25(0.87$ to 1.79$)$ \\
\hline Stillbirth rate & $5(3)$ & $4(2)$ & $2.27(0.62$ to 8.34$)$ & NA \\
\hline Neonatal mortality & $12(9)$ & $1(<1)$ & $22.27(2.93$ to 169.52$)$ & $29 \cdot 18(0.60$ to 1424.68$)$ \\
\hline
\end{tabular}

TABLE IV-Summary of differences between preterm and term eclampsia

^Relative risk of factor for woman with antepartum eclampsia.

\begin{tabular}{|c|c|c|c|c|}
\hline Factor of interest & $\begin{array}{c}\text { No }(\%) \text { of } \\
\text { preterm cases } \\
(n=169)\end{array}$ & $\begin{array}{l}\text { No }(\%) \text { of } \\
\text { term cases } \\
(n=214)\end{array}$ & $\begin{array}{l}\text { Relative risk }^{\star} \\
\text { (95\% confidence interval) }\end{array}$ & $\begin{array}{c}\begin{array}{c}\text { Summary relative risk } \\
\text { with stratified analysis } \\
\text { (corrected for type of } \\
\text { seizure) }\end{array} \\
(95 \% \text { confidence interval) }\end{array}$ \\
\hline $\begin{array}{l}\text { Prodromal symptoms } \\
\text { Prodromal signs ( } 325 \text { seen within past seven days) } \\
\text { Antepartum seizures } \\
\text { Seizure outside hospital } \\
\text { Multiple seizures } \\
\text { Delivered by caesarean section } \\
\text { Major maternal complication } \\
\text { Maternal death } \\
\text { Small for gestational age } \\
\text { Stillbirth rate } \\
\text { Neonatal mortality }\end{array}$ & $\begin{array}{r}124(76) \\
113(90) \\
111(66) \\
65(38) \\
81(49) \\
134(79) \\
79(47) \\
4(2) \\
78(50) \\
8(5) \\
12(7)\end{array}$ & $\begin{array}{l}103(48) \\
176(88) \\
36(17) \\
23(11) \\
77(36) \\
72(34) \\
56(26) \\
3(1) \\
22(11) \\
1(<1) \\
1(<1)\end{array}$ & $\begin{array}{c}1.58(1.34 \text { to } 1.86) \\
1.03(0.95 \text { to } 1.11) \\
3.90(2.84 \text { to } 5.36) \\
3.60(2.34 \text { to } 5.54) \\
1.35(1.06 \text { to } 1.71) \\
2.36(1.92 \text { to } 2.89) \\
1.80(1.36 \text { to } 2.37) \\
1.69(0.38 \text { to } 7.44) \\
4.53(2.96 \text { to } 6.92) \\
10.45(1.32 \text { to } 82.82) \\
16.36(2.15 \text { to } 124.58)\end{array}$ & $\begin{array}{c}1.42(1.18 \text { to } 1.71) \\
\text { NA } \\
\text { NA } \\
1.12(0.76 \text { to } 1.65) \\
1.10(0.83 \text { to } 1.44) \\
1.43(1.22 \text { to } 1.67) \\
1.38(1.02 \text { to } 1.85) \\
\text { NA } \\
4 \cdot 18(2.49 \text { to } 7.03) \\
13.27(0.91 \text { to } 193.31) \\
12.49(0.40 \text { to } 394.03)\end{array}$ \\
\hline
\end{tabular}

NSD=No significant difference. NA=Not applicable.

*Relative risk of factor for woman with preterm eclampsia.

relation between type of eclampsia and gestational age at presentation, stratified analyses were performed to determine which factors were independently associated. Multiple seizures were associated with antepartum eclampsia but not with gestational immaturity, whereas small for gestational age fetuses were associated with gestational immaturity but not the type of eclampsia. The presence of prodromal symptoms, one or more major maternal complications, and delivery by caesarean section were associated independently with both preterm and antepartum eclampsia (tables III and IV).

\section{Discussion}

This is the first comprehensive study of eclampsia in the United Kingdom. The total ascertainment together with the completeness of the data collection on maternal death mean that the incidence rates and maternal case fatalities for eclampsia are likely to be accurate.

The incidence of $4.9 / 10000$ is similar to that reported for the United States (4.3/10000 in 1983-6) ${ }^{9}$ but higher than that in Sweden $\left(2 \cdot 7 / 10000\right.$ in $\left.1980^{7}\right)$. Our inclusion criteria were similar to those used in the American study ${ }^{9}$ whereas the Swedish criteria were broader. Thus, the difference in incidence between Sweden and the two other countries may be greater.

\section{DECLINING INCIDENCE}

The incidence of eclampsia in the United Kingdom has declined since 1922 (when the rate was $80 / 10000^{3}$ ), but the evidence for any substantial decrease in the past 30 years is less compelling. Analysis of regional incidences suggests that there has been little if any improvement over the past two decades. There were 5.3 cases per 10000 deliveries among residents of South Glamorgan in 1965-74' ${ }^{10}$ compared with $4 \cdot 0 / 10000$ maternities for all of Wales in this study. In the Grampian region of Scotland in 1978-83 the rate was $8 / 10000^{11}$ compared with our data for the whole of
Scotland of 6.7/10000. Criteria for eclampsia were not explicitly stated in the above studies, but from the limited information provided it is clear that we would have excluded some of their cases.

In New Zealand eclampsia was a notifiable disease for many years, and its incidence declined from $32 / 10000$ to $8 / 10000$ between $1928-33$ and $1956-8^{12}$-a reduction Corkhill attributed to the advent of routine screening for early signs of pre-eclampsia. ${ }^{12}$ The reduction in incidence in the United Kingdom between the 1920s and 1970s also occurred as antenatal care became universally available. This, together with the fact that countries without effective antenatal screening programmes still have much higher incidence rates, ${ }^{13-15}$ provides circumstantial evidence to support Corkhill's hypothesis.

Most cases in this series, however, occurred despite a normal frequency of antenatal assessments $(70 \%)$ and even after admission to hospital (77\%). Furthermore, eclampsia was often (38\%) unheralded by hypertension and proteinuria, even in women in hospital, so it is not surprising that the convulsions could not be prevented. Unheralded eclampsia has been noted before but in proportionally fewer cases (about one in six)..$^{16} 17$

Corkhill's hypothesis and our findings are compatible. Screening by measurement of blood pressure and analysis of urine may prevent most eclampsia preceded by hypertension and proteinuria (evidenced by the reduction in incidence in the United Kingdom between 1922 and 1970). As eclampsia with a classic presentation is prevented, however, atypical presentations become a proportionately greater problem (hence the relatively stable incidence since the 1970 s and the clinical findings in this study).

The implications are that to achieve further substantial reductions in the incidence of eclampsia we need to develop new screening and diagnostic tests for features other than hypertension and proteinuria. Meanwhile, the development of more effective management should help to reduce the impact and complications of eclampsia when it does occur. The 
multicentre trial currently being analysed should provide some soundly based guidance on treatment for eclampsia. ${ }^{18}$

\section{SECONDARY PREVENTION}

Our study has measured the maternal case fatality of eclampsia in the United Kingdom for the first time. The figure of just under one in $\mathbf{5 0}$ shows that eclampsia is still a marker of an extremely dangerous disorder. It is not, however, the convulsions themselves that are hazardous (idiopathic epilepsy has a much lower case fatality) but the severity of the underlying disturbances. Therefore the focus of secondary prevention should not be simply to prevent convulsions in a desperate situation but to prevent the dangerous state itself.

Hypertension and proteinuria are not the only nor necessarily the most important signs of preeclampsia. ${ }^{19}$ Renal function tests, thrombocytopenia, and abnormal plasma concentrations of liver enzymes give important information about the extent to which the maternal system is affected. Monitoring these indices can give early warning of impending decompensations such as the HELLP (haemolysis, elevated liver enzymes and low platelets) syndrome $e^{20}$ or renal failure, as emphasised in reports of the confidential inquiries into maternal mortality. ${ }^{2}$ It was therefore disappointing that biochemical tests were often not done even after the onset of convulsions.

It is important to note that proteinuria was the only premonitory sign in $10 \%$ of cases and that one third of women had only mild hypertension before the onset of convulsions. In these cases there were often delays in making a diagnosis, apparently because of the belief that eclampsia "should" be associated with high blood pressures. Eclampsia is still commonly perceived as the end of a linear spectrum that stretches from normal pregnancy, through mild hypertension, proteinuric pre-eclampsia, and finally eclampsia, so that convulsions are not expected until the woman has severe hypertension, proteinuria, and symptoms.

An alternative view (consistent with current theories of pathogenesis) is that seizures are one of a range of signs and symptoms caused by widespread endothelial cell damage secondary to an ischaemic placenta. ${ }^{1}$ The initial signs and symptoms of the syndrome are therefore dictated by the site and extent of endothelial cell damage and not by a predetermined hierarchy. It is then logical that seizures may precede hypertension or proteinuria. In other words, the term pre-eclampsia is misleading because eclampsia can precede preeclampsia. Furthermore, just as hypertension may be the only manifestation of the pre-eclampsia syndrome, seizures may occur without other signs and still be a marker of the same underlying pathological process.

\section{CONCLUSIONS}

In conclusion, eclampsia complicates nearly one in 2000 pregnancies in the United Kingdom; nearly one in 50 affected women die as do one in 14 of their offspring. As the average case load per consultant drops to well below 1000 deliveries a year individual doctors can expect to see this complication too infrequently to acquire competence in its diagnosis and management. It would therefore seem desirable that at least one consultant in every unit takes a special interest in the problem. There is also the need for support from regional centres as outlined in the recent confidential inquiries into maternal deaths. ${ }^{21}$

Most eclamptic convulsions occur in hospital, and they may be unheralded by warning signs or symptoms. Preterm and antenatal eclampsia seem to be particularly dangerous. Every obstetric unit would benefit from having a well established protocol for coping with presentations of convulsions in pregnancy to avoid

\section{Clinical implications}

- Eclampsia complicates nearly one in 2000 pregnancies in the United Kingdom; nearly one in 50 affected women die of the condition as do one in 14 of their offspring

- Preterm and antenatal eclampsia seem to be particularly dangerous to both mother and fetus

- This study shows that most eclamptic convulsions in the United Kingdom occur in hospital in women who have received antenatal care

- Eclamptic seizures are not always predated by the common warning signs of pre-eclampsia; in particular, women may fit at relatively low diastolic blood pressures

- Every obstetric unit should have a well established protocol for coping with eclampsia to avoid delays in the diagnosis and management

delays in diagnosis and management. Confirmatory signs should be sought assiduously in the first 48 hours after a fit even if there are no features of preeclampsia previously. The signs should include disturbances of liver and coagulation function as well as new hypertension and proteinuria. Screening for hypertension and proteinuria has provided a simple and effective way of reducing the dangers of preeclampsia and eclampsia in the past, but new methods need to be sought to reduce the impact of the residual problems not detected by these signs.

This study was funded by the medical audit unit of the Royal College of Obstetricians and Gynaecologists. KAD was supported by the Reginald Walker Postgraduate Fellowship of the University of Adelaide, Australia. We thank Helen Temple, Marion Hall, Mike Maresh, Iain Chalmers, Adrian Grant, Lelia Duley, David Jewell, and Chris Mount for advice and help in establishing the study.

The study was possible only because of the participation of over 1000 obstetricians and several hundred midwives and general practitioners throughout the United Kingdom.

1 Roberts JM, Redman CWG. Pre-eclampsia: more than pregnancy-induced hypertension. Lancet 1993;341:1447-51.

2 Department of Health, Welsh Office, Scottish Home and Health Department, Department of Health and Social Services Northern Ireland. Report on confidential enquiries into maternal deaths in the United Kingdom 1985-87. London: HMSO, 1991.

3 Eden TW. Eclampsia. Fournal of Obstetrics and Gynaecology of the British Empire 1922;29:386-401.

4 Douglas KA, Redman CWG. Eclampsia in the United Kingdom. The "BEST"

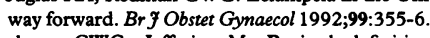

5 Redman CWG, Jefferies M. Revised definition of pre-eclampsia. Lancet 1988;i:809-12.

6 Yudkin P, Aboualfa M, Eyre J, Redman CWG, Wilkinson A. New birthweigh and head circumference centiles for gestational ages 24 to 42 weeks. Early Hum Dev 1987;15:45-52.

7 Moller B, Lindmark G. Eclampsia in Sweden, 1976-1980. Acta Obstet Gynecol Scand 1986;65:307-14.

8 Lopez Lera M. Main clinical types and subtypes of eclampsia. Am $\mathcal{f}$ Obstet Gynecol 1992;166:4-9.

9 Saftlas AF, Olson DR, Franks AL, Atrash HK, Pokras R. Epidemiology of preeclampsia and eclampsia in the United States, 1979-1986. Am ₹ Obstet Grecol 1990;163:460-5.

10 Wightman H, Hibbard BM, Rosen M. Perinatal mortality and morbidity associated with eclampsia. $B M f$ 1978;ii:235-7.

11 Hall $M$, Campbell D. Cost-effectiveness of present programs for detection of asymptomatic hypertension in relation to the severity of hypertension and proteinuric hypertension. Int $f$ Technol Assess Health Care 1992; 8(suppl 1):75-81.

12 Corkill TF. Experience of toxaemia control in Australia and New Zealand. Pathology and Microbiology 1961;24:428-34.

13 Moodley J, Naicker RS, Mankowitz E. Eclampsia-a method of managemen A preliminary report. $S$ Afr Med f 1983;63:530-5.

14 Porapakkham S. An epidemiologic study of eclampsia. Obstet Gynecol 1979;54:26-30.

15 Moore PJ, Munoz WP. Eclampsia in the black population of the Natal midlands. S Afr Med $\mathcal{F}$ 1985;67:597-9.

16 Sibai BM, Abdella TN, Spinnato JA, Anderson GD. Eclampsia V. The incidence of nonpreventable eclampsia. Am f Obstet Gynecol 1986;154: $581-6$. 
17 Campbell DM, Templeton AA. Is eclampsia preventable? In: Bonnar J, MacGillivray I, Symonds EM, eds. Pregnancy hypertension. Baltimore: University Park Press, 1980:483-8.

18 Duley L. Trial to compare the effects of magnesium sulphate and diazepam in the management of eclampsia, on maternal mortality and serious morbidity In: Chalmers I, ed. Oxford database of perinatal trials. Disk issue 7: record number 6561. Oxford: 1992.

19 Redman CWG, Roberts J. Management of pre-eclampsia. Lancet 1993;341
20 Weinstein L. Syndrome of hemolysis, elevated liver enzymes, and low platelet count. A severe consequence of hypertension in pregnancy. $A m \mathcal{f}$ Obstet Gynecol 1982;142:159-67.

21 Department of Health, Welsh Office, Scottish Home and Health Department, Department of Health and Social Services Northern Ireland. Report on confidential enquiries into maternal deaths in the United Kingdom 1988-90. London: HMSO, 1994

\title{
Midwife managed delivery unit: a randomised controlled comparison with consultant led care
}

\author{
V A Hundley, F M Cruickshank, G D Lang, C M A Glazener, J M Milne, M Turner, D Blyth, \\ J Mollison, C Donaldson
}

Department of Obstetrics and Gynaecology, University of Aberdeen, Aberdeen Maternity Hospital, Aberdeen AB9 2ZA

V A Hundley, research fellow G D Lang, consultant obstetrician

D Blyth, research assistant M Turner, project assistant

Aberdeen Royal Hospitals NHS Trust, Aberdeen AB9 2ZA

F M Cruickshank, research sister, Aberdeen Maternity Hospital

J M Milne, nurse manager, directorate of obstetrics and gynaecology

Health Services Research Unit, University of Aberdeen, Foresterhill, Aberdeen AB9 2ZD C M A Glazener, Wellcome research fellow in postnatal care

J Mollison, research assistant

\section{Health Economics}

Research Unit, Department of Public Health, University of Aberdeen, Foresterhill, Aberdeen AB9 2ZD

C Donaldson, deputy director

Correspondence to:

Ms Hundley.

\section{Abstract}

Objective-To examine whether intrapartum care and delivery of low risk women in a midwife managed delivery unit differs from that in a consultant led labour ward.

Design-Pragmatic randomised controlled trial. Subjects were randomised in a 2:1 ratio between the midwives unit and the labour ward.

Setting-Aberdeen Maternity Hospital, Grampian.

Subjects-2844 low risk women, as defined by existing booking criteria for general practitioner units in Grampian. 1900 women were randomised to the midwives unit and 944 to the labour ward.

Main outcome measures-Maternal and perinatal morbidity.

Results-Of the women randomised to the midwives unit, $647(34 \%)$ were transferred to the labour ward antepartum, $303(16 \%)$ were transferred intrapartum, and $80(4 \%)$ were lost to follow up. 870 women $(46 \%)$ were delivered in the midwives unit. Primigravid women (255/596, 43\%) were significantly more likely to be transferred intrapartum than multigravid women $(48 / 577,8 \%)$. Significant differences between the midwives unit and labour ward were found in monitoring, fetal distress, analgesia, mobility, and use of episiotomy. There were no significant differences in mode of delivery or fetal outcome.

Conclusions-Midwife managed intrapartum care for low risk women results in more mobility and less intervention with no increase in neonatal morbidity. However, the high rate of transfer shows that antenatal criteria are unable to determine who will remain at low risk throughout pregnancy and labour.

\section{Introduction}

If women are to have choice in the location for their delivery, the maternity services must provide a safe and acceptable range of options. In Aberdeen we have developed a midwife managed delivery unit that aims to offer women choice, participation, and control in their labour. Over the past 40 years in Britain women have had less choice as the proportion of babies delivered in consultant maternity units has increased and maternity services have moved away from community based delivery. It has been argued that hospital delivery provides greater safety for mother and baby ${ }^{1-3}$ but some researchers disagree.

This debate on the place of delivery and its safety is not new, but it has intensified in the past two years with the publication of recent reports and policy documents. ${ }^{5-7}$ Most would agree that close supervision and monitoring of high risk pregnancies is beneficial. However, the application of the same criteria to low risk pregnancies has been questioned. There is some evidence to suggest that there is more intervention in labour and greater maternal morbidity if a low risk woman is cared for in a consultant maternity unit rather than in a general practitioner unit $^{8-14}$ or by midwives in a birth room. ${ }^{15-17}$ Yet in many of these studies the sample populations have been small or not directly comparable. In all but three of the studies ${ }^{15-17}$ selection bias may have been introduced due to preference for a particular type of care. The experience of the family birthing unit in Melbourne showed that intrapartum problems do occur in low risk women, ${ }^{15}$ thus highlighting the importance of the close proximity of specialist obstetric, anaesthetic, and neonatal services. Further evaluation of alternative methods of obstetric care, in particular midwife managed care, is required. In this paper we report the results of one such evaluation of a midwifery managed delivery unit in the Aberdeen Maternity Hospital.

\section{Methods}

BACKGROUND

The midwives unit in Aberdeen was established in April 1990. It is a separate unit, of five single rooms, located 20 yards from the consultant led labour ward. The philosophy of care behind the unit is to provide a safe, "homely" environment where women can retain choice and control in the management of their labour. Midwives take total responsibility for the care delivered, thus developing and maintaining their competence. Labour is managed traditionally-the fetal heart rate is monitored with a Pinard stethoscope or hand held Doppler apparatus, active labour is encouraged, and there is minimal intervention. The unit is staffed and run by hospital midwives who work throughout the delivery suite according to clinical need. There is no input to the midwives unit by medical staff. However, the unit caters solely for low risk women and there are strict protocols for booking, admission, and transfer.

\section{STUDY AIMS}

The main objective was to compare care and delivery of low risk women in a midwife managed delivery unit with care and delivery in the consultant led labour ward in terms of four sorts of outcomes. As well as maternal and perinatal morbidity, reported here, we looked at the expectations, experiences, and satisfaction of parturient women; the role, experiences, and satisfaction of midwifery staff, and costs of care. These other outcomes will be reported elsewhere.

\section{STUDY POPULATION}

Low risk women were identified from general practitioners' referral letters. The exclusion criteria for the 
of cardiac death. That would be, as Bassan writes, the height of folly. Instead we recommended further evaluation of patients in whom monitoring performed for clinical reasons shows abnormal findings (described in our paper). As we wrote, the benefit of managing silent ischaemia in elderly people is unclear. Nevertheless, if silent ischaemia is diagnosed it is clinically and ethically accepted, even in elderly patients, that risk factors should be controlled and treatment with aspirin and even $\beta$ blockers considered. Specific treatment of the marker, arrhythmia, would be unwise and certainly ineffective. An important question is whether these markers are also valid in middle aged people.

Bassan argues that the markers were defined arbitrarily. This is not true. All clinically relevant arrhythmias shown by Holter monitoring were included in the analysis, and the cut off points for abnormal findings were based on the criteria used in other studies attempting to find prognostic markers.

Department of Geriatrics,

Senior lecturer

\section{University of Turku, \\ SF-20700 Turku, \\ Finland}

1 Bassan M. Continuous ambulatory electrocardiography in elderly people. $B M F$ 1995;310:468. (18 February.)

2 Räihä I, Piha J, Seppänen A, Puukka P, Sourander L. Predictive value of continuous ambulatory electrocardiographic monitorvalue of continuous ambulatory electrocardiographic mon
ing in the elderly. BMf 1994;309:1263-7. (12 November.)

\section{The persistent vegetative state}

\section{Task force's definition influences interpretation of outcome}

EDrToR,-Robin S Howard and David H Miller's comments on the study by the American MultiSociety Task Force on the persistent vegetative state leaves two aspects of the prognosis that need to be clarified. ${ }^{.}$The adult mortality of $82 \%$ at three years and $95 \%$ at five years might suggest that more prolonged survival in a vegetative state is uncommon. Yet it is the prospect of long survival that promotes the debate about continuing treatment. The task force, however, defined a persistent vegetative state as being in a vegetative state at only one month after an acute cerebral insult; this would include some patients who had been vegetative for only a few days after a period in coma. Many such patients die in the next few months and some recover, leaving only $59 \%, 37 \%$, and $24 \%$ of those vegetative at one month still alive and vegetative at three, six, and 12 months. Death rates reported for patients vegetative at three months in $\mathrm{Japan}^{23}$ and at six months in the Netherlands ${ }^{4}$ were much lower, while the death rate for those still vegetative at one year is quite low but not yet accurately documented.

With regard to recovery, the proportion of head injured adults who recover consciousness after three months in a vegetative state but who are severely disabled is more than half, not one fifth as Howard and Miller state. ${ }^{1}$ Just how severely disabled they may be is evident from Andrews's report of 11 recoveries after four months or more in a vegetative state. ${ }^{5}$ Only four patients could speak, only four could feed themselves, and five still needed a gastrostomy.

BRYAN JENNETT Professor emeritus of neurosurgery Institute of Neurological Sciences, Southern General Hospital, Glasgow G51 4TF

1 Howard RS, Miller DH. The persistent vegetative state. BMf 1995;310:341-2. (11 February.)

2 Higashi K, Sakata M, Hatano M, Abiko S, Ihara K, Katayama S, et al. Epidemiology studies on patients with a persisten vegetative state. ₹ Neurol Neurosurg Psychiatry 1977;40:876-85.
3 Higashi K, Hatano M, Abiko S, Thara K, Katayama S, Wakuta $\mathrm{Y}$, et al. Five year follow-up study of the patients with
persistent vegetative state. $\mathcal{f}$ Neurol Neurosurg Psychiatry 1981;44:552-4.

4 Minderhoud JM, Braakman R. Het vegeterende bestaan. Ned Tijdschr Geneeskd 1985;129:2385-8.

5 Andrews K. Recovery of patients after four months or more in the persistent vegetative state. $B M F$ 1993;306:1597-600.

\section{Report is unnecessarily negative}

EDrToR,-Robin S Howard and David H Miller's editorial on the persistent vegetative state' is timely because several working parties are debating this condition, including the Royal College of Physicians; the European Union's biomedical and health research programme (BIOMED I) at the Centre of Medical Law and Ethics at King's College, London; and an international working party and conference on management of the condition held at the Royal College of Physicians in March. The editorial reviews the position of the Multi-Society Task Force. ${ }^{23}$ Considerable concern has been expressed, particularly among consultants in rehabilitation, that the task force's report is unnecessarily negative.

The international working party was set up because of concern that insufficient attention was being given to the treatment, especially the rehabilitation, of patients in a persistent vegetative state. It will debate the concern about the terminology used; the classification of patients into various levels of persistent vegetative state and states of low awareness; the need for more sensitive measurement scales; the effectiveness of treatment programmes; and the relevance of rehabilitation for medical, legal, and social policy. Concern must be expressed at the assumption made by many people that severe disability is not worth achieving. This has implications for the treatment not only of people with severe brain damage but also of those with other chronically disabling disorders.

The persistent vegetative state is so rare that it is difficult, even in specialist units such as the Persistent Vegetative State Rehabilitation Unit in Putney, to build up sufficient research data to provide answers to many of the questions required to make reasonable clinical, legal, and social decisions on behalf of such unfortunate patients and their families.

Royal Hospital and Home, Putney,

London SW15 3SW

1 Howard RS, Miller DH. The persistent vegetative state. $B M f$ 1995;310:341-2. (11 February.)

2 Multi-Society Task Force on PVS. Medical aspects of persistent vegetative state (first part). N Engl f Med 1994;330:1499-508.

3 Multi-Society Task Force on PVS. Medical aspects of persistent vegetative state (second part). N Engl F Med 1994;330:1572-9.

\section{Eclampsia in the United Kingdom}

\section{More information is required}

EDITOR,-K A Douglas and C W G Redman have produced an invaluable 70 year landmark report on the state of eclampsia in the United Kingdom.' Hopefully, more information will follow as some aspects are not discussed in detail in their paper.

What of the $138(26 \%)$ women who were not included in the survey, having originally seemed to fit the criteria? What did these women with pseudoeclampsia have? Five of the 64 who had a fit while receiving community based care had hypertension and albuminuria, but how many had one or the other and how many had been seen in the week preceding the fit? The 356 singleton pregnancies had a fetal loss of $84 / 1000$, but the 55 babies in the 27 multiple pregnancies survived. Was the eclampsia different in these two groups- for example, in the incidence of major maternal complications? Roughly a quarter of the patient were multiparous, which suggests that primiparous women are more susceptible to the condition. To what extent is the different incidence of eclampsia in teenagers accounted for by the effect of parity? What are the relative risks of eclampsia in primigravidas and multigravidas? Was there any information about the multigravidas-for example, did they have new partners?

We find it difficult to understand the result under the heading "antenatal care." If one follows the standard antenatal care described in the article, 42 of the last 112 days of pregnancy are more than seven days away from the last visit-that is, $38 \%$ so one would expect to find that $62 \%$ of these patients had been seen in the week before their eclamptic fit. In fact, 325 (85\%) had been seen This is despite a quarter of the patients having either no or less than standard antenatal care. Had these patients been brought back early because, at the previous visit, they had had hypertension or proteinuria or some other cause for concern? Antenatal care as described so dramatically in the classic paper by Hamlin remains the cornerstone of prevention of eclampsia. ${ }^{2}$ Altogether $89 \%$ of these patients had warning signs. We desperately need a test for "dangerous" toxaemia.

Finally, was there any difference in postpartum eclampsia between vaginal delivery and caesarean section? Probably none of the women who had a caesarean section would have been given ergometrine, and they would have been the more severe cases. It would not surprise us, however, if the incidence of postpartum eclampsia was higher in patients who had few signs of toxaemia and had been given ergometrine.

\section{Consultant obsterrician and R H GOLDING \\ Consultant obstetrician and gynaecologist}

D G DANIEI

Royal Gwent Hospital

Newport,

Gwent NP9 2UB

1 Douglas KA, Redman CWG. Eclampsia in the United Kingdom. $B M F$ 1994;309:1395-400. (26 November.)

2 Hamlin RHJ. The prevention of eclampsia and pre-eclampsia. Lancet 1952;i:64-8.

\section{Better standards of care will reduce morbidity and mortality}

EDrToR,-Traditional teaching is that eclampsia reflects poor standards in obstetric practice, and $\mathrm{K}$ A Douglas and C W G Redman's paper provides further evidence of this. ${ }^{1}$ Of the 383 women with eclampsia, 294 were inpatients when eclampsia occurred and only 36 had no recorded hypertension or proteinuria in the week before the first fit.

Successive confidential inquiries into maternal mortality have highlighted the seriousness of this condition, identifying important substandard care in $88 \%$ of deaths. ${ }^{2}$ It is disappointing to learn that $23 \%$ of the women with eclampsia in this study had less than standard antenatal care and that biochemical tests were so poorly used: only $54 \%$ of the women had their plasma creatinine concentration measured and only $30 \%$ had their transaminase concentration measured. The most recent confidential inquiry noted that ergometrine had been given to five of 27 women dying of hypertension disorders of pregnancy. ${ }^{2}$ We would be interested to hear from the authors whether similar inappropriate use of ergometrine was an important factor in postpartum eclampsia in their study.

The authors found that in those women whose blood pressure was recorded within an hour of the onset of convulsions the mean diastolic pressure was $97 \mathrm{~mm} \mathrm{Hg}$. We wonder whether they found any difference in blood pressure between the women who had eclampsia antenatally and those 
who had it postnatally; we could find no references where this has been investigated previously.

The authors' analysis of risk factors highlights some common misconceptions: a quarter of all the women with eclampsia were parous, $18 \%$ of all the women with eclampsia were parous with no history of pre-eclampsia or eclampsia in previous pregnancies, and teenagers were at three times greater risk than older women. Overall, this paper is a sobering reminder of the vigilance required if we are to reduce further the morbidity and mortality from this relatively common condition.

\section{EDWARD O'DONNELL Registrar in obstetrics DAVID SOMERSET} Senior house officer in obstetrics Birmingham Maternity Hospital, Birmingham B15 2TG

1 Douglas KA, Redman CWG. Eclampsia in the United Kingdom. $B M F$ 1994;309:1395-400. (26 November.)

2 Department of Health, Welsh Office, Scottish Home and Health Department, Department of Health and Social Services Northern Ireland. Repon on confidential enquiries into maternal deaths in the United Kingdom 1988-1990. London: HMSO, 1994

\section{Authors' reply}

EdToR,-D G Daniel and R H Golding ask for more information on the women with eclampsia. Altogether 198 women were reported but excluded (60 after talking to consultants and 138 after the case note review) because they did not meet the criteria for the study. Seventy three of these had pre-eclampsia but no seizure; 56 had uncomplicated epilepsy; 40 had simple faints, hypoglycaemic fits, or pseudoseizures; and 29 had seizures without an apparent cause but did not have signs of pre-eclampsia.

Eighty nine women had a fit while under community based care, and for 64 of these we had access to a complete record of the care. Of the 64 women, six had hypertension alone, 11 had proteinuria alone, and five had hypertension and proteinuria documented before the onset of seizures. Twenty six of the 64 had been seen in the week preceding the fit.

The 27 women with multiple pregnancy were significantly less likely to have antepartum seizures than those with singleton pregnancies (relative risk ( $95 \%$ confidence interval) $0.18(0.05$ to 0.69$)$ ), but there were no significant differences between the two groups in terms of the presence of prodromal signs or symptoms, the gestation at onset of seizures, the number of seizures, or the ensuing maternal morbidity or mortality.

National data on number of previous stillbirths and live births do not exist, so that accurate calculation of rate of eclampsia in primiparous compared with multiparous women cannot be calculated accurately. Seventy one $(99 \%)$ of the teenagers were primiparous compared with 215 $(69 \%)$ of the women aged 20 or more. Part of the increased risk associated with teenage pregnancy may therefore be due to primiparity.

Whether or not the multigravidas had new partners was not determined, but $26(27 \%)$ had a history of pre-eclampsia and one $(1 \%)$ had a history of eclampsia. Multiparous women were significantly more likely to report prodromal symptoms than primiparous women (relative risk

Comparison of blood pressures in women with antepartum onset of seizures and women with intrapartum and postpartum onsets. Figures are means (SD)

\begin{tabular}{|c|c|c|c|}
\hline & $\begin{array}{l}\text { Antepartum } \\
\text { onset } \\
(\mathrm{n}=147)\end{array}$ & $\begin{array}{l}\text { Intrapartum and } \\
\text { postpartum onsets } \\
(n=236)\end{array}$ & Significance ${ }^{\star}$ \\
\hline $\begin{array}{l}\text { Last systolic pressure (taken within one hour of onset of seizures) }(\mathrm{mm} \mathrm{Hg}) \\
\text { Last diastolic pressure (taken within one hour of onset of seizures) }(\mathrm{mm} \mathrm{Hg}) \\
\text { Maximum recorded systolic pressure }(\mathrm{mmHg}) \\
\text { Maximum recorded diastolic pressure }(\mathrm{mm} \mathrm{Hg})\end{array}$ & $\begin{array}{l}166(26 \cdot 2) \\
105(14 \cdot 2) \\
185(26 \cdot 0) \\
120(13 \cdot 0)\end{array}$ & $\begin{array}{r}156(21 \cdot 2) \\
94(14 \cdot 0) \\
179(20 \cdot 7) \\
113(11 \cdot 6)\end{array}$ & $\begin{array}{r}5.67, \mathrm{P}=0.017 \\
18.05, \mathrm{P}<0.001 \\
4.51, \mathrm{P}=0.034 \\
26 \cdot 49, \mathrm{P}<0.001\end{array}$ \\
\hline
\end{tabular}

${ }^{\star}$ Kruskal-Wallis $H$ test (1 df).
$1 \cdot 31(1 \cdot 11$ to $1 \cdot 53))$, but there were no significant differences in outcome measures.

Daniel and Golding's analysis of antenatal care assumes that eclampsia was uniformly spread through the last 112 days of pregnancy. Seizures were more common in the last four weeks of pregnancy, when it is routine to see women weekly; this helps to explain the high proportion of women seen within seven days of their seizure. Other women may have been brought back early or sought medical advice about symptoms. Our data are not detailed enough to supply this information.

There were no significant differences in the presence of prodromal symptoms (relative risk $1.00(0.75$ to 1.33$))$ or signs $(1.08(1.0$ to 1.17$))$ between women who had postpartum eclampsia after caesarean section or vaginal delivery. We did not record whether ergometrine was used in the third stage of labour.

In answer to the questions raised by Edward O'Donnell and David Somerset, women with antepartum eclampsia had significantly higher systolic and diastolic blood pressures within one hour of the onset of seizures than those with postpartum seizures (table). This is consistent with the overall picture of antepartum eclampsia being a more severe condition.

KA DOUGLAS Visiting research fellow

C W G REDMAN

Professor of obstetric medicine

Nuffield Department of Obstetrics and Gynaecology,

Oxford OX3 9D

\section{Treating leg cramp}

\section{Naftidrofuryl is a safe and effective alternative}

EDITOR,-As part of their meta-analysis of the efficacy of quinine in treating leg cramp Malcolm Man-Son-Hing and George Wells review the pharmacological alternatives to quinine for this painful condition. 'They fail to mention naftidrofuryl. In a double blind placebo controlled crossover study of cramp at rest we showed naftidrofuryl to produce both a significant reduction in the frequency of cramp and an increase in the number of days free of cramp. ${ }^{2}$ Man-SonHing and Wells point out the possible serious side effects of quinine, and we suggest that naftidrofuryl should be regarded as an alternative, potentially less hazardous treatment for cramp at rest.

We agree with Man-Son-Hing and Wells that the condition is extremely common; indeed, we have shown a prevalence of $37 \%$ in the elderly population overall. ${ }^{3}$ Such a high prevalence further emphasises the need to use treatment with a low profile of side effects.

\section{MARTIN J CONNOLLY} Senior lecture J B YOUNG

Department of Care of the Elderly, St Luke's Hospital,

JR NAYLOR

Huddersfield National Health Service Trust,

Huddersfield Royal Infirmary,

Huddersfield HD3 3EA
John Radcliffe Hospital,
Man-Son-Hing M, Wells G. Meta-analysis of efficacy of quinine for treatment of nocturnal leg cramps in elderly people. $B M Y$ 1995;310:13-7. (7 January.)

2 Young JB, Connolly MJ. Naftidrofuryl treatment for rest cramp. Postgrad Med ₹ 1993;69:624-6.

3 Naylor JR, Young JB. A general population survey of rest cramps. Age Ageing 1994;23:418-20.

The trial of naftidrofuryl ${ }^{2}$ received support from Lipha Pharmaceuticals, which provided the active drug and placebos, randomisation envelopes, and trial booklets to monitor patients recruited to the trial.

\section{Study quoted had flawed design}

EDITOR,-In their meta-analysis of the efficacy of quinine for night cramps Malcolm Man-Son-Hing and George Wells analysed six trials that met strict criteria for design. ${ }^{1}$ Included in these six trials is one carried out by one of us (NRD) in 1991 on patients from his practice and a practice in Southampton. ${ }^{2}$ It is surprising that the authors included the data from this trial, since it was stated clearly that the results showed that the design of the trial was invalid. As the table shows, there was a clear carryover effect: patients who received quinine first, followed by placebo, experienced more nights with cramp in the placebo period than did those who received the placebo first. The test for carry over is significant (Mann-Whitney $\mathrm{W}=247 \cdot 5, \mathrm{P}<0.05)$.

Proportion of nights with cramp

\begin{tabular}{lccc}
\hline Treatment & $\begin{array}{c}\text { 1st Period } \\
(\mathrm{n}=750)\end{array}$ & $\begin{array}{c}\text { 2nd Period } \\
(\mathrm{n}=750)\end{array}$ & $\begin{array}{c}\text { Both periods } \\
(\mathrm{n}=1500)\end{array}$ \\
\hline Quinine & $203(27)$ & $104(14)$ & $300(20)$ \\
Placebo & $278(37)$ & $488(65)$ & $765(51)$
\end{tabular}

Because of the carryover effect the only valid comparison was between the two treatments in the first period. The difference was not significant (Mann-Whitney $W=183, P=0 \cdot 37$ ). The use of the combined figures for both periods by the authors of the meta-analysis is therefore not legitimate. The results from this trial suggest that the withdrawal of quinine after four weeks' treatment tends to induce leg cramps. This effect is dificult to explain and has not been reported elsewhere.

N R DUNN

Poole,

General practitioner

Dorset BH18 8BQ

Department of Medical Statistics,

Southampton General Hospital,

Southampton SO9 4XY

1 Man-Song-Hing M, Wells G. Meta-analysis of efficacy of quinine for treatment of nocturnal leg cramps in elderly people. $B M$ 1995;310:13-7. (7 January.)

2 Dunn NR. Effectiveness of quinine for night cramps. $\mathrm{Br} \mathcal{f} \mathrm{Gen}$ Pract 1993;43:127-8.

\section{Protection afforded by cycle helmets}

EDITOR,-According to Richard Keatinge and Ruth Parry, we "defy engineering evidence [unspecified] in stating that cycle helmets reduce the risk of serious head injury in accidents involving motor vehicles." They take us to task for not having mentioned the paper of Spaite et al. ${ }^{2}$ We referred to this paper in our report in $1993,{ }^{3}$ which we referenced in the letter to which Keatinge and Parry are responding. ${ }^{4}$ Spaite et al found that injuries to the head and body were less severe in helmeted riders. Sex and age were separately associated with use of a helmet, severity of injury, and mortality. In these circumstances a more sophisticated analysis was warranted, and Spaite et al's conclusion that helmet wearing was simply a 


\section{BIBLIOGRAPHY}

Anonymous. (1990) Medical ethics: should medicine turn the other cheek? (editorial). Lancet. 2: 846-7.

Anonymous. The Directory of Emergency and Special Care Units 1991. Cambridge: CMA Medical Data Ltd., 1991:

Anonymous. (1993) Low-dose aspirin in prevention and treatment of intrauterine growth retardation and pregnancy-induced hypertension. Italian study of aspirin in pregnancy. Lancet. 341: 396-400.

Abramson JH. Making sense of data. A self-instruction manual on the interpretation of epidemiological data. Oxford: Oxford University Press, 1988.

Adelusi B, Ojengbede OA. (1986) Reproductive performance after eclampsia. Int J Gynaecol Obstet. 24: 183-9.

Adetoro OO. (1990) The pattern of eclampsia at the University of Ilorin Teaching Hospital (U.I.T.H.) Ilorin, Nigeria. Int J Gynaecol Obstet. 31: 221-6.

Agobe JT, Good W, Hancock KW. (1981) Meteorological relations of eclampsia in Lagos. Nigeria. Br J Obstet Gynaecol. 88: 706-10.

Alderman BW, Boyko EJ, Loy GL, Jones RH, Keane EM, Daling JR. (1988) Weather and occurrence of eclampsia. Int J Epidemiol. 17: 582-8.

Altman.D. Practical Statistics for Medical Research. London: Chapman \& Hall, 1991.

American College of Obstetricians and Gynecologists. (1986) Management of preeclampsia. ACOG Technical Bulletin. 91.

Appleton MP, Kuehl TJ, Raebel MA, Adams HR, Knight AB, Gold WR. (1991) Magnesium sulfate versus phenytion for seizure prophylaxis in pregnancy-induced hypertension. Am J Obstet Gynecol. 165: 907-13.

ASSHIP. Management of Hypertension in Pregnancy. Consensus Statement. Australian Society for the Study of Hypertension in Pregnancy (ASSHIP), 1993: 1-46.

ASSHIP. (1993) Management of hypertension in pregnancy: executive summary. Med J Aust. 158: 700-2.

Attewell R. Multivariate Analysis (Logistic regression). Course Notes from the National Centre for Epidemiology and Population Health. Unpublished work. 
Backe B, Nakling J. (1993) Effectiveness of antenatal care; a population based study. Br J Obstet Gynaecol. 100: 727-32.

Baird D. (1977) Epidemiological aspects of hypertensive pregnancy. Clin Obstet Gynaecol. 4: 531-48.

Beaufils M, Uzan S, Donsimoni R, Colau JC. (1985) Prevention of pre-eclampsia by early antiplatelet therapy. Lancet. i: 840-2.

Belfort MA, Carpenter RJ, Kirshon B, Saade GR, Moise KJ. (1993) The use of nimodipine in a patient with eclampsia: Color flow Doppler demonstration of retinal artery relaxation. Am J Obstet Gynecol. 169: 204-6.

Belizan JM, Villar J, Repke J. (1988) The relationship between calcium intake and pregnancy-induced hypertension: up-to-date evidence [see comments]. Am J Obstet Gynecol. 158: 898-902.

Bell ET, Dieckmann W, Eastman NJ. (1940) Classification of the toxemias of pregnancy. The Mother. April.

Bhose L. (1964) Postpartum Eclampsia. Am J Obstet Gynecol. 89: 898-902.

Boyd PA, Lindenbaum RH, Redman C. (1987) Pre-eclampsia and trisomy 13: a possible association. Lancet. 2: 425-7.

BPSU. The British Paediatric Surveillance Unit - Third Annual Report 1988-89. London: BPSU, 1989: 1-18.

Bracken MB, Srisuphan W. (1982) Oral contraception as a risk factor for preeclampsia. Am J Obstet Gynecol. 142: 191-6.

Brown CE, Purdy P, Cunningham FG. (1988) Head computed tomographic scans in women with eclampsia. Am J Obstet Gynecol. 159: 915-20.

Browne FJ. (1933) The early signs of pre-eclampsia toxaemia. with special reference to the order of their appearance, and their interrelation. J Obstet Gynaecol Br Empire. 40: 1160 .

Bryans CI. (1966) The remote prognosis in toxemia of pregnancy. Clin Obstet Gynecol. 9: 973-90.

Butler NR, Alberman ER. Perinatal problems. In: The second report of the 1958 British Perinatal Mortality Survey. Edinburgh: Livingstone, 1969:36-8.

Campbell DM, MacGillivray I, Carr Hill R, Samphier M. (1983) Fetal sex and preeclampsia in primigravidae. Br J Obstet Gynaecol. 90: 26-7. 
Campbell DM, MacGillivray I, Carr Hill R. (1985) Pre-eclampsia in second pregnancy. Br J Obstet Gynaecol. 92: 131-40.

Campbell DM, Templeton AA. Is eclampsia preventable?. In: Bonnar J, MacGillivray I, Symonds EM, eds. Pregnancy Hypertension. Baltimore: University Park Press, 1980:4838.

Carleton H, Forsythe A, Flores R. (1988) Remote prognosis of preeclampsia in women 25 years old and younger. Am J Obstet Gynecol. 159: 156-60.

Chatterjee TK, Hickey MU, Chikamta DM. (1978) A review of 79 cases of eclampsia at University Teaching Hospital, Lusaka. Med J Zambia. 12: 77-80.

Chesley LC, Annitto JE, Cosgrove RA. (1968) The familial factor in toxemia of pregnancy. Obstet Gynecol. 32: 303-11.

Chesley LC. (1976) Blood pressure, edema and proteinuria in pregnancy. 1. Historical developments. Prog Clin Biol Res. 7: 19-66.

Chesley LC, Annitto JE, Cosgrove RA. (1976) The remote prognosis of eclamptic women. Sixth periodic report. Am J Obstet Gynecol. 124: 446-59.

Chesley LC. History. In: Chesley LC, ed. Hypertensive disorders in pregnancy. AppletonCentury-Crofts, 1978a:17-34.

Chesley LC. Remote prognosis. In: Chesley LC, ed. Hypertensive disorders in pregnancy. New York: Appleton-Century-Crofts, 1978b:421.

Chesley LC. Evolution of concepts of eclampsia. In: Bonnar J, MacGillivray I, Symonds EM, eds. Pregnancy Hypertension. Baltimore: University Park Press, 1980:3-11.

Chesley LC. (1984) Habitus and eclampsia. Obstet Gynecol. 64: 315-8.

Chesley LC, Cooper DW. (1986) Genetics of hypertension in pregnancy: possible single gene control of pre-eclampsia and eclampsia in the descendants of eclamptic women. $\mathrm{Br} \mathrm{J}$ Obstet Gynaecol. 93: 898-908.

Chua S, Redman CW. (1991) Are prophylactic anticonvulsants required in severe preeclampsia? [letter]. Lancet. 337: 250-1.

CLASP Collaberative Group. (1994) CLASP: a randomised trial of low-dose aspirin for the prevention and treatment of pre-eclampsia among 9364 pregnant women. Lancet. 343: 619-29. 
Collins R, Wallenburg HCS. Pharmacological prevention and treatment of hypertensive disorders in pregnancy. In: Chalmers I, Enkine M, Keirse MJNC, eds. Effective care in pregnancy and childbirth. Oxford: Oxford University Press, 1989:512-33.

Colosimo C, Jr., Fileni A, Moschini M, Guerrini P. (1985) CT findings in eclampsia. Neuroradiology. 27: 313-7.

Cooper DW, Hill JA, Chesley LC, Bryans CI. (1988) Genetic control of susceptibility to eclampsia and miscarriage. Br J Obstet Gynaecol. 95: 644-53.

Corkill TF. (1961) Experience of toxaemia control in Australia and New Zealand. Path Microbiol. 24: 428-34.

Crawford S, Varner MW, Digre KB, Servais G, Corbett JJ. (1987) Cranial magnetic resonance imaging in eclampsia. Obstet Gynecol. 70: 474-7.

Crowther C. (1990) Magnesium sulphate versus diazepam in the management of eclampsia: a randomized controlled trial. Br J Obstet Gynaecol. 97: 110-7.

Crowther CA. (1985a) Eclampsia at Harare Maternity Hospital. An epidemiological study. S Afr Med J. 68: 927-9.

Crowther CA. (1985b) Management and pregnancy outcome in eclampsia at Harare Maternity Hospital. Cent Afr J Med. 31: 107-9.

Cummins CJ. (1973) Review of maternal deaths in New South Wales from eclampsia and severe preeclampsia--1961 to 1969 . Med J Aust. 1: 342-5.

Davey DA, MacGillivray I. (1988) The classification and definition of the hypertensive disorders of pregnancy [see comments]. Am J Obstet Gynecol. 158: 892-8.

Davies AM, Czaczkes JW, Sadovsky E, Prywes R, Weiskopf P, Sterk VV. (1970) Toxemia of Pregnancy in Jerusalem Epidemiological Studies of a Total Community. Israel Journal of Medical Sciences. 6 No 2: 253-66.

Davies AM. (1971) Geographical epidemiology of the toxemias of pregnancy. Isr J Med Sci. 7: 751-821.

Davies AM, Poznansky R, Weiskopf P, Prywes R, Sadovsky E, Czaczkes W. (1976) Toxemia of pregnancy in Jerusalem. II. The role of diet. Isr J Med Sci. 12: 509-18.

Dean AG, Dean JA, Burton AH, Dicker RC. Epi Info. Verson 5; a word processing. database, and statistics program for epidemiology on microcomputers. Atlanta, Georgia, USA: 1990. 
Department of Finance and Personel. Seventy-first annual report of the Registrar General 1992. Belfast: HMSO, 1994.

Dhall K. (1984) Epidemiology of preeclampsia and eclampsia. Asia Oceania J Obstet Gynaecol. 10: 89-93.

DHSS, Welsh Office, Scottish Home and Health Department, Department of Health and Social Services Northern Ireland. Report on Confidential Enquiries into Maternal Deaths in the United Kingdom 1985-87. London: HMSO, 1991.

DHSS, Welsh Office, Scottish Home and Health Department, Department of Health and Social Services Northern Ireland. Report on Confidential Enquiries into Maternal Deaths in the United Kingdom 1988-90. London: HMSO, 1994.

DHSS, Welsh Office. Report on Confidential Enquiries into Maternal Deaths in England and Wales 1982-84. London: HMSO, 1989.

Dinsdale HB. (1988) Does magnesium sulfate treat eclamptic seizures? Yes. Arch Neurol. 45: $1360-1$.

Doll R, Hanington E. (1961) International Survey of Eclampsia and pre-Eclampsia. 195859: Epidemiological Aspects. Path Microbiol. 24: 531-41.

Dommisse J. (1990) Phenytoin sodium and magnesium sulphate in the management of eclampsia. Br J Obstet Gynaecol. 97: 104-9.

Donaldson JO. (1986) Does magnesium sulfate treat eclamptic convulsions? Clin Neuropharmacol. 9: 37-45.

Donaldson JO. Eclampsia. In: Neurology of Pregnancy. 2nd ed. W.B. Saunders and Co., 1989:269-310.

Douglas KA, Redman CW. (1992) Eclampsia in the United Kingdom. The 'BEST' way forward. Br J Obstet Gynaecol. 99: 355-6.

Duffus GM, MacGillivray I. (1968) The incidence of pre-eclamptic toxaemia in smokers and non-smokers. Lancet. 1: 994-5.

Duley L. (1992a) Maternal mortality associated with hypertensive disorders of pregnancy in Africa, Asia, Latin America and the Caribbean. Br J Obstet Gynaecol. 99: 547-53.

Duley L. Trial to compare the effects of magnesium sulphate and diazepam in the management of eclampsia, on maternal mortality and serious morbidity. In: Chalmers I, ed.Oxford database of perinatal trials. disk issue 7: record number $6561,1992 \mathrm{~b}$. 
Dunn R, Lee W, Cotton DB. (1986) Evaluation by computerized axial tomography of eclamptic women with seizures refractory to magnesium sulfate therapy. Am J Obstet Gynecol. 155: 267-8.

Eastman NJ, Bell ET, Dieckmann WJ. (1952) Definition and classification of toxemias brought up-to-date. American Committee on Maternal Welfare, Chicago.

Eden TW. (1922) Eclampsia. J Obstet Gynaecol Br Empire. 29: 386-401.

Eskenazi B, Fenster L, Sidney S. (1991) A multivariate analysis of risk factors for preeclampsia. JAMA. 266: 237-41.

Friedman SA, Taylor RN, Roberts JM. (1991) Pathophysiology of preeclampsia. Clin Perinatol. 18: 661-82.

Gedekoh RH, Hayashi TT, MacDonald HM. (1981) Eclampsia at Magee-Womens Hospital. 1970 to 1980. Am J Obstet Gynecol. 140: 860-6.

Goodlin RC. (1988) Cerebral vasopasm and eclampsia [letter]. Stroke. 19: 1446.

Goven ADT. (1961) The pathogenesis of eclamptic lesions. Path Microbiol. 24: 561.

Government statistical service. Annual Report of the Registrar General for Scotland 1992. Edinburgh: General Register Office, 1993: 1-143.

Hall M, Campbell D. (1992) Cost-effectiveness of present programs for detection of asymptomatic hypertension in relation to the severity of hypertension and proteinuric hypertension. Int J Technol Assess Health Care. 8 Suppl 1: 75-81.

Hall MH, Chng PK, MacGillivray I. (1980) Is routine antenatal care worth while? Lancet. 2: 78-80.

Hargood JL, Brown MA. (1991) Pregnancy-induced hypertension: recurrence rate in second pregnancies. Med J Aust. 154: 376-7.

Hauch E, Lehmann K. (1934) Investigations into the occurrence of eclampsia in Denmark during the years 1918-27. Acta Obstet Gynecol Scand. 14: 425-81.

Hennekens CH, Buring JE. Epidemiology in Medicine. Boston: Little, Brown and Company, 1987.

Hibbard BM. (1978) Pre-eclampsia/eclampsia. Practitioner. 221: 847-53.

Hogberg U, Joelsson I. (1985) The decline in maternal mortality in Sweden, 1931-1980. Acta Obstet Gynecol Scand. 64: 583-92. 
Horn EH, Filshie M, Kerslake RW, Jaspan T, Worthington BS, Rubin PC. (1990) Widespread cerebral ischaemia treated with nimodipine in a patient with eclampsia. $\mathrm{Br}$ Med J. 301: 794.

Hughes EC. Obstetric-gynecologic terminology. Philadelphia: Davis, 1972: 422-3.

Hutton JD, James DK, Stirrat GM, Douglas KA, Redman CW. (1992) Management of severe pre-eclampsia and eclampsia by UK consultants. Br J Obstet Gynaecol. 99: 554-6.

Imperiale TF, Petrulis AS. (1991) A meta-analysis of low-dose aspirin for the prevention of pregnancy-induced hypertensive disease. JAMA. 266: 260-4.

Jeffcoate TN, Scott JS. (1959) Some observations on the placental factor in pregnancy toxaemia. Am J Obstet Gynecol. 77: 475-89.

Jeffcoate TNA, Scott JS. (1959) Some observations on the placental factor in pregnancy toxemia. Am J Obstet Gynecol. 77: 475.

Kaplan PW, Lesser RP, Fisher RS, Repke JT, Hanley DF. (1988) No, magnesium sulfate should not be used in treating eclamptic seizures. Arch Neurol. 45: 1361-4.

Knox EG. (1992) Confidential medical records and epidemiological research. Br Med J. 304: 727-8.

Konje JC, Obisesan KA, Odukoya OA, Ladipo OA. (1992) Presentation and management of eclampsia. Int J Gynaecol Obstet. 38: 31-5.

Lewis PJ, Bulpitt CJ, Zuspan FP. (1980) A comparison of current British and American practice in the management of hypertension in pregnancy. J Obstet Gynaecol. 1980: 7882.

Liang ST, Yam A, Ma HK. (1984) Changing trends in the clinical features of eclampsia. Asia Oceania J Obstet Gynaecol. 10: 457-64.

Lindberg BS, Sandstrom B. (1981) How Swedish obstetricians manage hypertension in pregnancy. A questionnaire study. Acta Obstet Gynecol Scand. 60: 327-31.

Liston WA, Kilpatrick DC. (1991) Is genetic susceptibility to pre-eclampsia conferred by homozygosity for the same single recessive gene in mother and fetus?. Br J Obstet Gynaecol. 98: 1079-86.

Lopez Llera M. (1967) Eclampsia 1963-1966. Evaluation of the treatment of 107 cases. J Obstet Gynaecol Br Commonw. 74: 379-84.

Lopez Llera M, Hernandez Horta J, Huttich F. (1972) Retarded fetal growth in eclampsia. J Reprod Med. 9: 229-32. 
Lopez Llera M, Rubio Linares G, Hernandez Horta JL. (1976) Maternal mortality rates in eclampsia. Am J Obstet Gynecol. 124: 149-55.

Lopez Llera M. (1982) Complicated eclampsia: fifteen years' experience in a referral medical center. Am J Obstet Gynecol. 142: 28-35.

Lopez Llera M, De la Luna Olsen E, Niz Ramos J. (1989) Eclampsia in twin pregnancy. J Reprod Med. 34: 802-6.

Lopez Llera M. (1990) Eclampsia and fetal sex. Int J Gynaecol Obstet. 33: 211-3.

Lopez Llera M. (1992) Main clinical types and subtypes of eclampsia. Am J Obstet Gynecol. 166: 4-9.

Lopez Llera M, Hernandez Horta JL. (1974) Pregnancy after eclampsia. Am J Obstet Gynecol. 119: 193-8.

Loudon I. (1991) Some historical aspects of toxaemia of pregnancy. A review. Br J Obstet Gynaecol. 98: 853-8.

MacGillivray I. (1958) Some Observations on the Incidence of Pre-Eclampsia. J Obstet Gynaecol Br Empire. 65: 536-9.

MacGillivray I. Epidemiology of pre-eclampsia and eclampsia. In: Pre-Eclampsia The hypertensive disease of pregnancy. W B Saunders Co. Ltd. UK, 1983a:13-22.

MacGillivray I. Definitions and Classifications. In: Pre-Eclampsia The Hypertensive Disease of Pregnancy. UK: WB Saunders Co Ltd, 1983b:1-12.

MacGillivray I. Factors Predisposing to the development of pre-eclampsia. In: PreEclampsia The Hypertensive disease of Pregnancy. W B Saunders Co. Ltd. UK, 1983c:23-55.

Macintosh RR. (1952) The significance of fits in eclampsia. J Obstet Gynaecol Br Empire. 59: 197-201.

Marcoux S, Brisson J, Fabia J. (1989a) The effect of cigarette smoking on the risk of preeclampsia and gestational hypertension. Am J Epidemiol. 130: 950-7.

Marcoux S, Brisson J, Fabia J. (1989b) The effect of leisure time physical activity on the risk of pre-eclampsia and gestational hypertension. J Epidemiol Community Health. 43: 147-52.

Marcoux S, Berube S, Brisson J, Fabia J. (1992) History of migraine and risk of pregnancy-induced hypertension. Epidemiology. 3: 53-6. 
McFarlane A, Scott JS. (1976) Pre-eclampsia/eclampsia in twin pregnancies. J Med Genet. 13: 208-11.

Medical Research Council of New Zealand. (1962) Report on Eclampsia. N Z Med J. July: 362-3.

Mehta L, Young ID. (1987) Recurrence risks for common complications of pregnancy--a review. Obstet Gynecol Surv. 42: 218-23.

Miles JF, Jr., Martin JN, Jr., Blake PG, Perry KG, Jr., Martin RW, Meeks GR. (1990) Postpartum eclampsia: a recurring perinatal dilemma. Obstet Gynecol. 76: 328-31.

Milliez J, Dahoun A, Boudraa M. (1990) Computed tomography of the brain in eclampsia. Obstet Gynecol. 75: 975-80.

Mills JL, Klebanoff MA, Graubard BI, Carey JC, Berendes HW. (1991) Barrier contraceptive methods and preeclampsia. JAMA. 265: 70-3.

Moller B, Lindmark G. (1986) Eclampsia in Sweden, 1976-1980. Acta Obstet Gynecol Scand. 65: 307-14.

Moodie P. (1992) The role of local research ethics committees. Br Med J. 304: 1129-30.

Moodley J, Naicker RS, Mankowitz E. (1983) Eclampsia--a method of management. A preliminary report. S Afr Med J. 63: 530-5.

Moore PJ, Munoz WP. (1985) Eclampsia in the black population of the Natal midlands. S Afr Med J. 67: 597-9.

Mphahlele M. (1975) Clinical experience of eclampsia at the University Teaching Hospital. Lusaka. An analysis of 50 cases. S Afr Med J. 49: 82-4.

Nalliah S, Abdullah AR. (1990) Eclampsia in Kelantan. Med J Malaysia. 45: 49-56.

National High Blood Pressure Education Program Working Group. (1990) Report on High Blood Pressure in Pregnancy. Am J Obstet Gynecol. 163: 1691-712.

Nelson TR. (1955a) A Clinical Study of Pre-Eclampsia Part 2. J Obstet Gynaecol Br Empire. 62: 58-66.

Nelson TR. (1955b) A Clinical Study of Pre-Eclampsia Part 1. J Obstet Gynaecol Br Empire. 62: 48-57.

Neutra R. (1973) A case-control study for estimating the risk of eclampsia in Cali. Colombia. Am J Obstet Gynecol. 117: 894-903. 
Neutra R. (1974) Meteorological factors and eclampsia. J Obstet Gynaecol Br Commonw. 81: 833-40.

Neutra R. (1975) Fetal death in eclampsia: I. Its relation to low gestational age, retarded fetal growth and low birthweight. Br J Obstet Gynaecol. 82: 382-9.

Neutra R, Neff R. (1975) Fetal death in eclampsia: II. The effect of non-therapeutic factors. Br J Obstet Gynaecol. 82: 390-6.

Newman RB, Eddy GL. (1988) Association of eclampsia and hydatidiform mole: case report and review of the literature. Obstet Gynecol Surv. 43: 185-90.

Odum CU, Akinkugbe A. (1991) The causes of maternal deaths in eclampsia in Lagos. Nigeria. West Afr J Med. 10: 371-6.

Olsen SF, Secher NJ. (1990) A possible preventive effect of low-dose fish oil on early delivery and pre-eclampsia: indications from a 50-year-old controlled trial. Br J Nutr. 64: 599-609.

OPCS. Birth Statistics: Review of the Registrar General on births and patterns of family building in England and Wales, 1991. London: HMSO, 1993:

OPCS. Birth statistics Review of the Registrar General on births and patterns of family building in England and Wales, 1992. London: HMSO, 1994:

Page EW. (1948) Placental dysfunction in eclamptogenic toxemias. Obstet Gynecol Surv. 3: 615-28.

Paterson WG, Hobson BM, Smart GE, Bain AD. (1971) Two cases of hydatidiform degeneration of the placenta with fetl abnormality and triploid chromosome constitution. $J$ Obstet Gynaecol Br Commonw. 78: 136-42.

Perry KG, Jr., Martin JNJ. (1992) Abnormal hemostasis and coagulopathy in preeclampsia and eclampsia. Clin Obstet Gynecol. 35: 338-50.

Peterkin IR, Wee R, Desmarais RL. (1992) Reversible cerebral, hepatic and renal lesions in severe pre-eclampsia. Can Assoc Radiol J. 43: 60-3.

Porapakkham S. (1979) An epidemiologic study of eclampsia. Obstet Gynecol. 54: 26-30.

Pritchard JA, Cunningham FG, Pritchard SA. (1984) The Parkland Memorial Hospital protocol for treatment of eclampsia: evaluation of 245 cases. Am J Obstet Gynecol. 148: 951-63.

Pritchard JA, Pritchard SA. (1975) Standardized treatment of 154 consecutive cases of eclampsia. Am J Obstet Gynecol. 123: 543-52. 
Raman L, Venkatesh PR. Pattern of Pre-Eclampsia and Eclampsia in Bangalore Hospitals in India. In: Sammour MB, Symonds EM, Zuspan FP, Tomi NE, eds. Pregnancy Hypertension. Abbassia Cairo: Ain Shams University Press, 1982:39-41.

Ramsay MM, Rimoy GH, Rubin PC. (1994) Are anticonvulsants necessary to prevent eclampsia?. Lancet. 343: 540-1.

Raps EC, Galetta SL, Broderick M, Atlas SW. (1993) Delayed peripartum vasculopathy: cerebral eclampsia revisited. Ann Neurol. 33: 222-5.

Raroque HGJ. (1989) Cerebral vasospasm in eclampsia [letter]. Stroke. 20: 826

Rauramo L. (1961) The incidence of eclampsia in Finland 1927-1958. Path Microbiol. 24: 435-43.

Redman CWG, Roberts J . (1993) Management of pre-eclampsia. Lancet. 341: 1451-4.

Redman CW. (1988) Eclampsia still kills. Br Med J Clin Res Ed. 296: 1209-10.

Redman CW. (1991) Current topic: pre-eclampsia and the placenta. Placenta. 12: 301-8.

Redman CW, Bonnar J. (1978) Plasma urate changes in pre-eclampsia. Br Med J. 1: 14845.

Redman CW, Jefferies M. (1988) Revised definition of pre-eclampsia. Lancet. 1: 809-12.

Redman CWG. Hypertension in pregnancy. In: de Swiet M, ed. Medical Disorders of Pregnancy. 2nd ed. Oxford: Blackwells Scientific Publications, 1989:249-305.

Roberts JM. Pregnancy related hypertension. In: Creasy RK, Resnik R, eds. Maternal Fetal Medicine; Principles \& practice. 2nd ed. W.B. Saunders and Company, 1989:777823.

Roberts JM, Taylor RN, Musci TJ, Rodgers GM, Hubel CA, McLaughlin MK. (1989) Preeclampsia: an endothelial cell disorder. Am J Obstet Gynecol. 161: 1200-4.

Roberts JM, Redman CWG. (1993) Pre-eclampsia: more than pregnancy-induced hypertension. Lancet. 341: 1447-51.

Robson SC, Walkinshaw S, Redfrern N, De Swiet M, Rodeck C. (1990) Seizure prophylaxis using phenytion in severe pre-eclampsia and eclampsia. J Obstet Gynaecol. 10: 449-50.

Rosa FW, Bosco LA, Graham CF, Milstien JB, Dreis M, Creamer J. (1989) Neonatal anuria with maternal angiotensin-converting enzyme inhibition. Obstet Gynecol. 74: 3714. 
Rosenfield A. (1992) Maternal mortality: community-based interventions. Int J Gynaecol Obstet. 38 Suppl: S17-22.

Rubin PC. (1986) Treatment of hypertension in pregnancy. Clin Obstet Gynaecol. 13: 307-17.

Sadeh M. (1989) Action of magnesium sulfate in the treatment of preeclampsia-eclampsia. Stroke. 20: 1273-5.

Saftlas AF, Olson DR, Franks AL, Atrash HK, Pokras R. (1990) Epidemiology of preeclampsia and eclampsia in the United States. 1979-1986. Am J Obstet Gynecol. 163: 460-5.

Samuels B. (1960) Postpartum Eclampsia.. Obstet Gynecol. 15: 748-52.

Sanders TG, Clayman DA, Sanchez Ramos L, Vines FS, Russo L. (1991) Brain in eclampsia: MR imaging with clinical correlation. Radiology. 180: 475-8.

Sheehan HL, Lynch JB. Pathology of Toxaemia of Pregnancy. Edinburgh: Churchill Livingstone, 1973.

Sibai BM, Schneider JM, Morrison JC, et al. (1980) The late postpartum eclampsia controversy. Obstet Gynecol. 55: 74-8.

Sibai BM, McCubbin JH, Anderson GD, Lipshitz J, Dilts PVJ. (1981) Eclampsia. I. Observations from 67 recent cases. Obstet Gynecol. 58: 609-13.

Sibai BM, Anderson GD, Abdella TN, McCubbin JH, Dilts PVJ. (1983) Eclampsia. III. Neonatal outcome, growth, and development. Am J Obstet Gynecol. 146: 307-16.

Sibai BM, Spinnato JA, Watson DL, Lewis JA, Anderson GD. (1985) Eclampsia. IV. Neurological findings and future outcome. Am J Obstet Gynecol. 152: 184-92.

Sibai BM, Abdella TN, Spinnato JA, Anderson GD. (1986a) Eclampsia. V. The incidence of nonpreventable eclampsia. Am J Obstet Gynecol. 154: 581-6.

Sibai BM, el Nazer A, Gonzalez Ruiz A. (1986b) Severe preeclampsia-eclampsia in young primigravid women: subsequent pregnancy outcome and remote prognosis. Am J Obstet Gynecol. 155: 1011-6.

Sibai BM. Eclampsia. In: Rubin PC, ed. Handbook of Hypertension. Volume 10, hypertension in pregnancy.. Elsevier Science Publishers, 1988:320-39.

Sibai BM. (1990a) Eclampsia. VI. Maternal-perinatal outcome in 254 consecutive cases. Am J Obstet Gynecol. 163: 1049-54. 
Sibai BM. (1990b) Magnesium sulfate is the ideal anticonvulsant in preeclampsiaeclampsia [see comments]. Am J Obstet Gynecol. 162: 1141-5.

Sibai BM, Mercer B, Sarinoglu C. (1991) Severe preeclampsia in the second trimester: recurrence risk and long-term prognosis. Am J Obstet Gynecol. 165: 1408-12.

Sibai BM, Sarinoglu C, Mercer BM. (1992) Eclampsia. VII. Pregnancy outcome after eclampsia and long-term prognosis. Am J Obstet Gynecol. 166: 1757-61.

Sibai BM, Caritis S, Phillips E, Klebanoff M, McNellis D, Rocco L. (1993) Prevention of pre-eclampsia: low-dose aspirin in nulliparous women: a double blind, placebo-controlled trial. Am J Obstet Gynecol. 167: 286.

Stevenson AC, Say B, Ustaoglu S, Durmus Z. (1976) Aspects of pre-eclamptic toxaemia of pregnancy, consanguinity, twinning in Ankara. J Med Genet. 13: 1-8.

Sumnulu I, Ildeniz M, Ozel N. (1989) The incidence of pregnancy induced hypertension in southeast Turkey. Int J Gynaecol Obstet. 28: 211-5.

Sutherland A, Cooper DW, Howie PW, Liston WA, MacGillivray I. (1981) The indicence of severe pre-eclampsia amongst mothers and mothers-in-law of pre-eclamptics and controls. Br J Obstet Gynaecol. 88: 785-91.

Swartjes JM, Schutte MF, Bleker OP. (1992) Management of eclampsia: cardiopulmonary arrest resulting from magnesium sulfate overdose. Eur J Obstet Gynecol Reprod Biol. 47: 73-5.

Templeton A, Campbell D. (1979) A retrospective study of eclampsia in the Grampian region, 1965--1977. Health Bull. 37: 55-9.

Trommer BL, Homer D, Mikhael MÂ. (1988) Cerebral vasospasm and eclampsia. Stroke. 19: $326-9$.

Trudinger BJ, Parik I. (1982) Attitudes to the management of hypertension in pregnancy: a survey of Australian fellows. Aust N Z J Obstet Gynaecol. 22: 191-7.

Villar MA, Sibai BM. (1988) Eclampsia: Obstet Gynecol Clin North Am. 15: 355-77.

Wallenburg HC, Dekker GA, Makovitz JW, Rotmans P. (1983) Low-dose aspirin prevents pregnancy-induced hypertension and pre-eclampsia in angiotensin-sensitive primigravidae. Lancet. i: 1-3.

Wallenburg HCS. Detecting hypertensive disorders of pregnancy. In: Chalmers 1, Enkin M, Keirse MJNC, eds. Effective care in Pregnancy and Childbirth. Oxford: Oxford University Press, 1989:382-402. 
Watson DL, Sibai BM, Shaver DC, Dacus JV, Anderson GD. (1983) Late postpartum eclampsia: an update. South Med J. 76: 1487-9.

Weinstein L. (1982) Syndrome of hemolysis, elevated liver enzymes, and low platelet count: A severe consequence of hypertension in pregnancy. Am J Obstet Gynecol. 142: 159-67.

Wightman H, Hibbard BM, Rosen M. (1978) Perinatal mortality and morbidity associated with eclampsia. Br Med J. 2: 235-7.

Will AD, Lewis LK. (1987) Cerebral vasoconstriction in toxaemia. Neurology. 37: 1555.

Yudkin P, Abouale, Eyre J, Redman CWG, Wilkinson A. (1987) Oxford standard charts for birthweight. Boys and girls 24-42 weeks. Early Human Development. 15: 45-52.

Zuspan FP, Ward MC. (1964) Treatment of Eclampsia. South Med J. 57: 954. 